\title{
Ni-Catalyzed Regio- and Enantioselective Domino Reductive Cyclization: One-Pot Synthesis of 2,3-Fused Cyclopentannulated Indolines
}

Yuanyuan Ping, ${ }^{\prime}$ Kuai Wang, ${ }^{\ddagger}$ Qi Pan, Zhengtian Ding, Zhijun Zhou, Ya Guo, and Wangqing Kong*

\author{
E-mail:wqkong@whu.edu.cn
}

The Center for Precision Synthesis, Institute for Advanced Studies, Wuhan University, 299 Bayi Road, Wuhan, Hubei 430072, P. R. China 


\section{Contents}

1. General information $\quad$ S3

2. General procedures $\quad$ S4

3. Optimization of reaction conditions $\quad$ S6

4. Control experiments $\quad$ S8

5. General procedure for the synthesis of starting materials $\quad$ S18

6. Characterization data of products $\quad$ S21

7. Crystallographic data for compound $3 \mathbf{k a}, 8 \mathbf{a a}$ and $(3 R, 8 S)-8 \mathbf{a a} \quad S 123$

8. Copies of the ${ }^{1} \mathrm{H},{ }^{19} \mathrm{~F}$ and ${ }^{13} \mathrm{C}$ NMR spectra $\quad \mathrm{S} 134$

$\begin{array}{ll}\text { 9. References } & \text { S196 }\end{array}$ 


\section{General Information}

${ }^{1} \mathrm{H}$ and ${ }^{13} \mathrm{C}$ NMR data were recorded with Bruker ADVANCE III $(400 \mathrm{MHz})$ or JNM$\mathrm{ECZ400S/L1}(400 \mathrm{MHz})$ spectrometers. Chemical shifts are given in ppm. The spectra are calibrated to the residual ${ }^{1} \mathrm{H}$ and ${ }^{13} \mathrm{C}$ signals of the solvents. Multiplicities are abbreviated as follows: singlet (s), doublet (d), triplet (t), quartet (q), doublet-doublet (dd), quintet (quint), septet (sept), multiplet ( $\mathrm{m}$ ), and broad (b). ${ }^{19} \mathrm{~F}$ NMR spectra were recorded using $\mathrm{CFCl}_{3}$ as internal standard. Gas chromatography were determined with a Varian GC 2000 gas chromatography instrument with a FID detector. High-resolution mass spectra (HRMS) were recorded on DIONEX UltiMate 3000 \& Bruker Compact TOF mass spectrometer. Enantiomeric excesses were determined with a SHIMADZU LC-20ADXR system using chiral stationary phase columns (DAICEL) by comparing the samples with the corresponding racemic samples. Column and elution details were specified in each entry. For the ReactIR kinetic experiments, the reaction spectra were recorded using an IC 10 and IC 15 from Mettler-Toledo AutoChem. Data manipulation was carried out using the iC IR software, version 4.2.

Materials and Methods: Unless otherwise stated, starting materials were purchased from commercial suppliers (Adamas-beta ${ }^{\circledR}$, Alfa, Aldrich and so on). $\mathrm{Ni}(\mathrm{COD})_{2}$ (Strem), Mn powder (Alfa Aesar-140+325 mesh, 99.6\% metals basis). All reactions dealing with air- or moisture-sensitive compounds were performed in the argon-filled glove box or by standard Schlenk techniques in oven-dried reaction vessels under argon atmosphere. Solvents were purchased in HPLC quality, degassed by purging thoroughly with argon and dried over activated molecular sieves of appropriate size. More sensitive compounds were stored in a desiccator or in a glove-box if required. Reactions were monitored by thin layer chromatography (TLC) using glass $0.25 \mathrm{~mm}$ silica gel plates. Compounds were visualized by UV-light at $254 \mathrm{~nm}$ and by dipping the plates in an aqueous potassium permanganate solution followed by heating. Flash column chromatography was performed over silica gel (200-400 mesh). 


\section{General Procedures}

2.1 General procedure for the cyclization reaction of arylbromide-tethered acrylamides 1 with alkynyl bromides 2 :

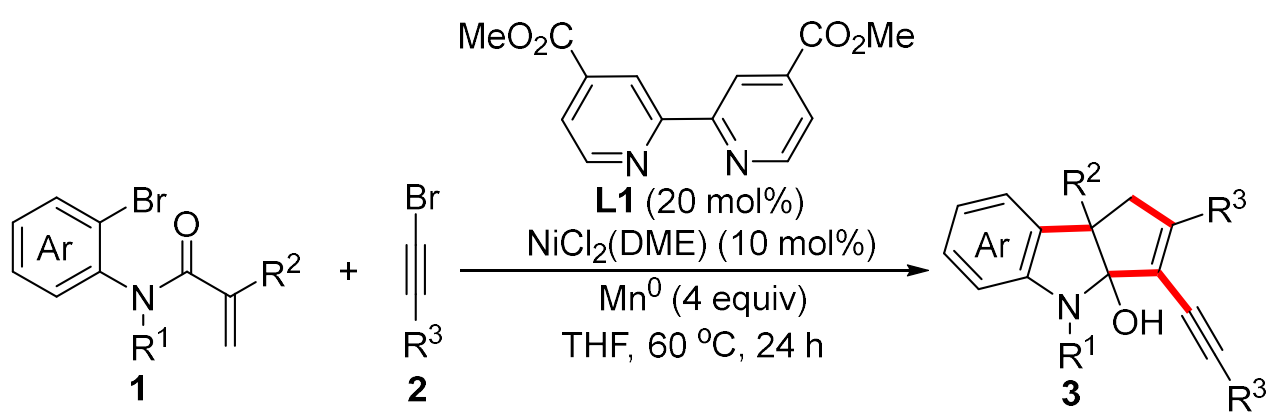

An oven-dried seal-tube equipped with a PTFE-coated stir bar was charged with alkene-tethered arylbromide $1(0.1 \mathrm{mmol})$ and $\mathbf{L 1}(20 \mathrm{~mol} \%)$, and brought into an argon filled glovebox. $\mathrm{NiCl}_{2}$ (DME) (10 mol\%), $\mathrm{Mn}^{0}$ (4 equiv), alkynyl bromide 2 (3 equiv) was added, followed by anhydrous THF $(0.05 \mathrm{M}, 2 \mathrm{~mL})$. The seal-tube was sealed and removed from the glovebox. Then the reaction was stirred at room temperature for $10 \mathrm{~min}$ and heated at $60{ }^{\circ} \mathrm{C}$ for $24 \mathrm{~h}$ until the reaction was complete (monitored by TLC). The solvent was then removed under vacuum and the residue was purified by chromatography on silica gel, eluting with ethyl acetate/toluene 1:40-1:5 to afford the desired indoline 3 .

2.2 General procedure for the cyclization reaction of arylbromide-tethered acrylamides 1 with alkynes 7:

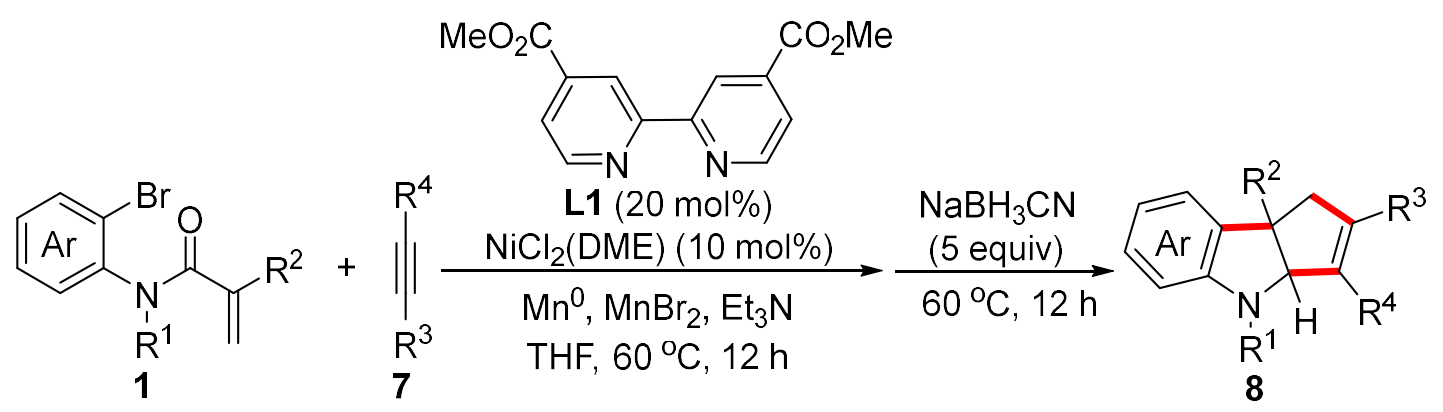

An oven-dried seal-tube equipped with a PTFE-coated stir bar was charged with alkene-tethered arylbromide $1(0.1 \mathrm{mmol})$ and $\mathbf{L 1}(20 \mathrm{~mol} \%)$, and brought into an argon filled glovebox. $\mathrm{NiCl}_{2}$ (DME) (10 mol\%), $\mathrm{Mn}^{0}$ (4 equiv), $\mathrm{MnBr}_{2}$ (1 equiv) and alkyne 7 (3 equiv) was added, followed by Et $3 \mathrm{~N}$ (2 equiv) and anhydrous THF (0.05 M, $2 \mathrm{~mL}$ ). The 
seal-tube was sealed and removed from the glovebox. Then the reaction was stirred at room temperature for $10 \mathrm{~min}$ and heated at $60^{\circ} \mathrm{C}$ for $12 \mathrm{~h}$ until the reaction was complete (monitored by TLC). $\mathrm{NaBH}_{3} \mathrm{CN}$ (5 equiv) was then added and stirred at $60^{\circ} \mathrm{C}$ for another 12 hour. The crude reaction mixture was filtered through a pad of silica and the solvent was removed under vacuum. The residue was then purified by chromatography on silica gel, eluting with petroleum ether/toluene 2:1-1:99 to afford the desired indoline 8 .

2.3 General procedure for the enantioselective cyclization reaction of arylbromidetethered acrylamides 1 with alkynes 7 :

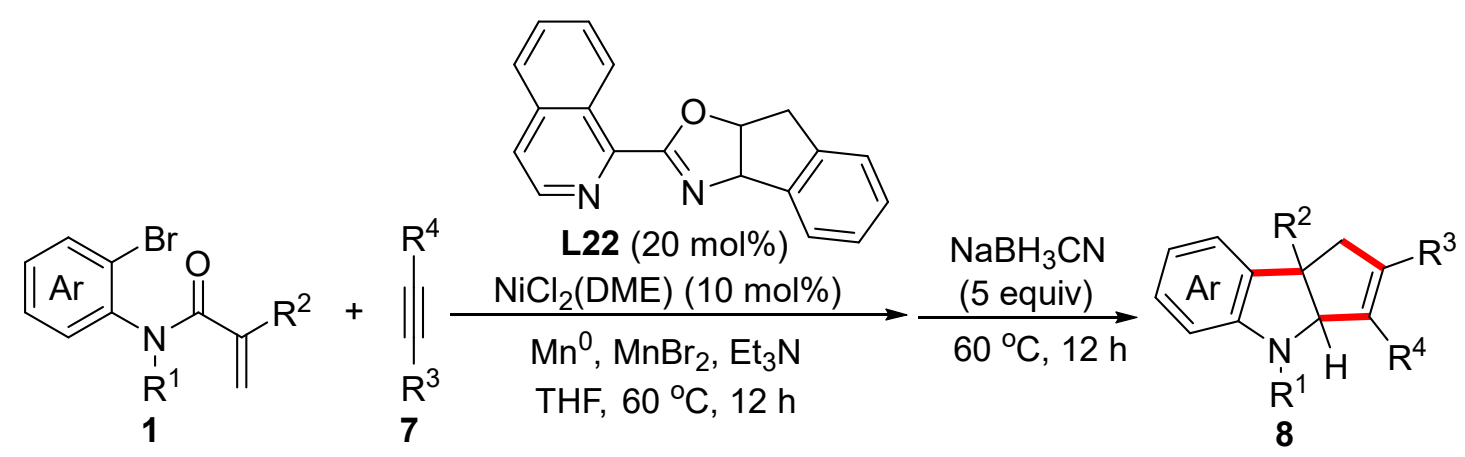

An oven-dried seal-tube equipped with a PTFE-coated stir bar was charged with alkene-tethered arylbromide $1(0.1 \mathrm{mmol})$ and L22 $(20 \mathrm{~mol} \%)$, and brought into an argon filled glovebox. $\mathrm{NiCl}_{2}$ (DME) (10 mol\%), $\mathrm{MnBr}_{2}$ (1 equiv), $\mathrm{Mn}^{0}$ (4 equiv) and alkyne 7 (3 equiv) was added, followed by Et3 $\mathrm{N}$ and anhydrous THF $(0.05 \mathrm{M}, 2 \mathrm{~mL})$. The seal-tube was sealed and removed from the glovebox. Then the reaction was stirred at room temperature for $10 \mathrm{~min}$ and heated at $60{ }^{\circ} \mathrm{C}$ for $12 \mathrm{~h}$ until the reaction was complete (monitored by TLC). $\mathrm{NaBH}_{3} \mathrm{CN}$ (5 equiv) was then added and stirred at $60^{\circ} \mathrm{C}$ for another 12 hour. The crude reaction mixture was filtered through a pad of silica and the solvent was removed under vacuum. The residue was then purified by chromatography on silica gel, eluting with petroleum ether/toluene 2:1-1:99 to afford the desired indoline 8. 


\section{Optimization of Reaction Conditions}

Table S1: Optimization of Reaction Conditions ${ }^{a}$

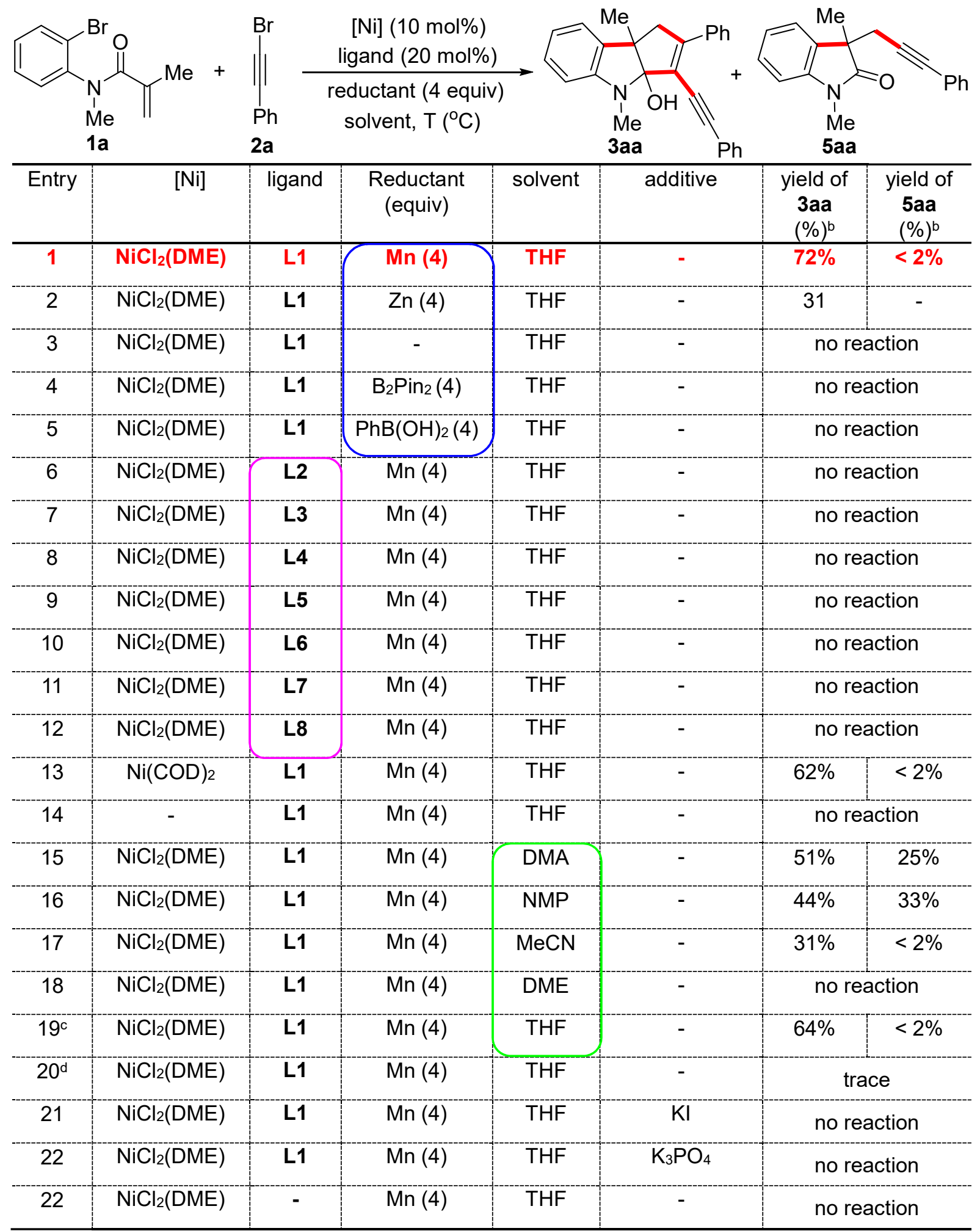



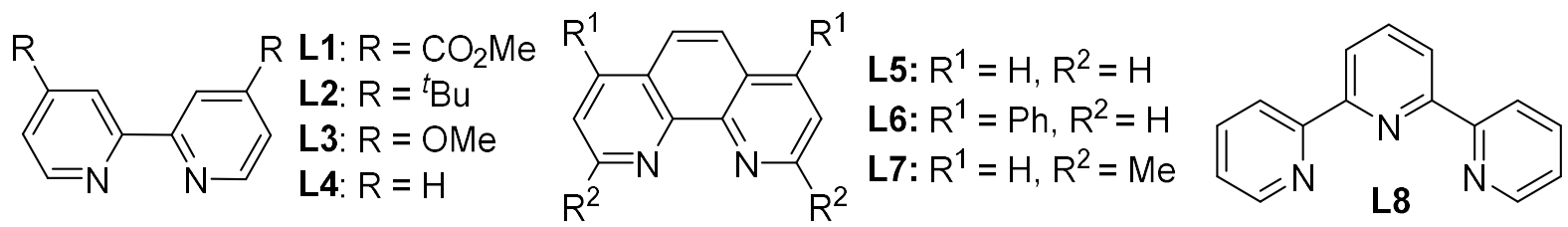

aReactions were carried out with $1 \mathrm{a}(0.1 \mathrm{mmol}), \mathbf{2 a}$ (3 equiv), $\mathrm{NiCl}_{2}(\mathrm{DME})(10 \mathrm{~mol} \%)$, ligand $(20 \mathrm{~mol} \%)$ and $\mathrm{Mn}^{0}(0.4 \mathrm{mmol})$ in $2 \mathrm{~mL}$ THF in a sealed tube at $60^{\circ} \mathrm{C}$ for $24 \mathrm{~h}$, unless noted otherwise. blsolated yields. ${ }^{\mathrm{c}} \mathbf{2 a}$ (4 equiv). ${ }^{\text {droom temperature. }}$ 


\section{Control Experiments}

\subsection{Evaluation of possibility of arylalkynylated product 5 as intermediate}

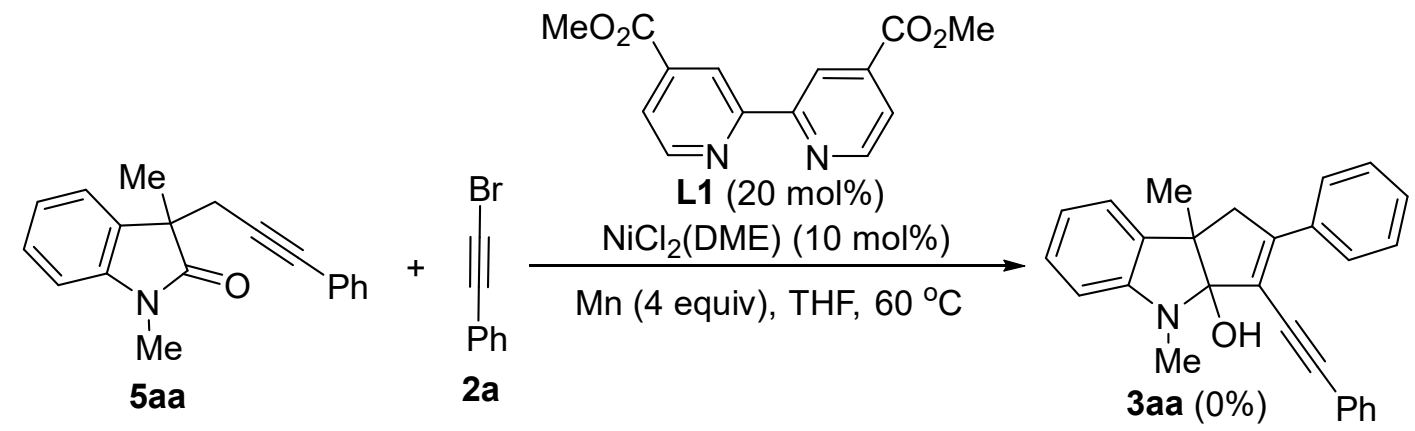

Experimental procedure: An oven-dried seal-tube equipped with a PTFE-coated stir bar was charged with $5 \mathrm{aa}(0.1 \mathrm{mmol}, 27.4 \mathrm{mg})$ and $\mathbf{L} 1(0.02 \mathrm{mmol}, 5.2 \mathrm{mg})$, and brought into an argon filled glovebox. $\mathrm{NiCl}_{2}(\mathrm{DME})(0.01 \mathrm{mmol}, 2.2 \mathrm{mg}), 2 \mathrm{a}(0.3 \mathrm{mmol}, 60.4 \mathrm{mg})$ and $\mathrm{Mn}^{0}(0.4 \mathrm{mmol}, 23.5 \mathrm{mg})$ was added, followed by anhydrous THF $(2 \mathrm{~mL})$. The tube was sealed and removed from the glovebox. Then the reaction was stirred at room temperature for $10 \mathrm{~min}$ and heated at $60^{\circ} \mathrm{C}$ for $24 \mathrm{~h}$. After removal of the solvent under reduced pressure, redissolved in $\mathrm{CDCl}_{3}$ and the crude mixture analyzed by ${ }^{1} \mathrm{H} \mathrm{NMR}$. The product 3aa was not detected.

Conclusions: This result argue againest the arylalkynylated product $\mathbf{5}$ as intermediate in our transformation. 


\subsection{Evaluation of possibility of 1,3-diyne 4 as intermediate}

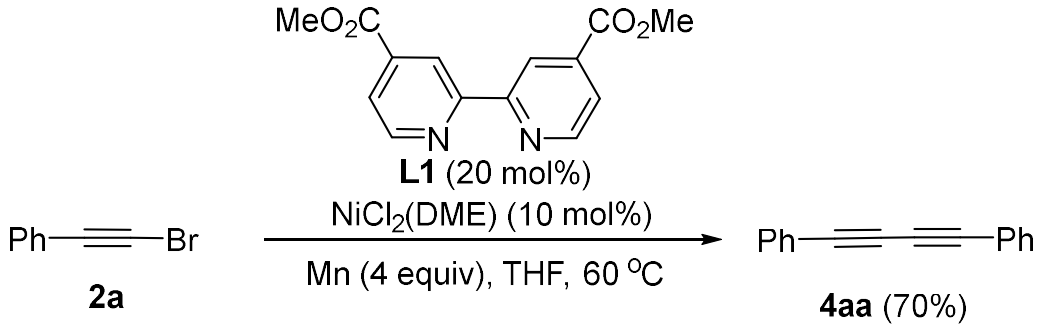

Experimental procedure: An oven-dried seal-tube equipped with a PTFE-coated stir bar was charged with $\mathbf{L} 1(0.02 \mathrm{mmol}, 5.4 \mathrm{mg})$ and brought into an argon filled glovebox. $\mathrm{NiCl}_{2}$ (DME) $(0.01 \mathrm{mmol}, 2.0 \mathrm{mg}), 2 \mathrm{a}(0.1 \mathrm{mmol}, 18.1 \mathrm{mg})$ and $\mathrm{Mn}^{0}(0.4 \mathrm{mmol}, 23.0 \mathrm{mg})$ was added, followed by anhydrous THF $(2 \mathrm{~mL})$. The tube was sealed and removed from the glovebox. Then the reaction was stirred at room temperature for $10 \mathrm{~min}$ and heated at $60{ }^{\circ} \mathrm{C}$ for $24 \mathrm{~h}$. The solvent was then removed under vacuum and the residue was purified by chromatography on silica gel, eluting with ethyl acetate/petroleum ether 1:1001:40 to afford 1,3-diyne 4 aa (14.1 mg, $70 \%$ ).

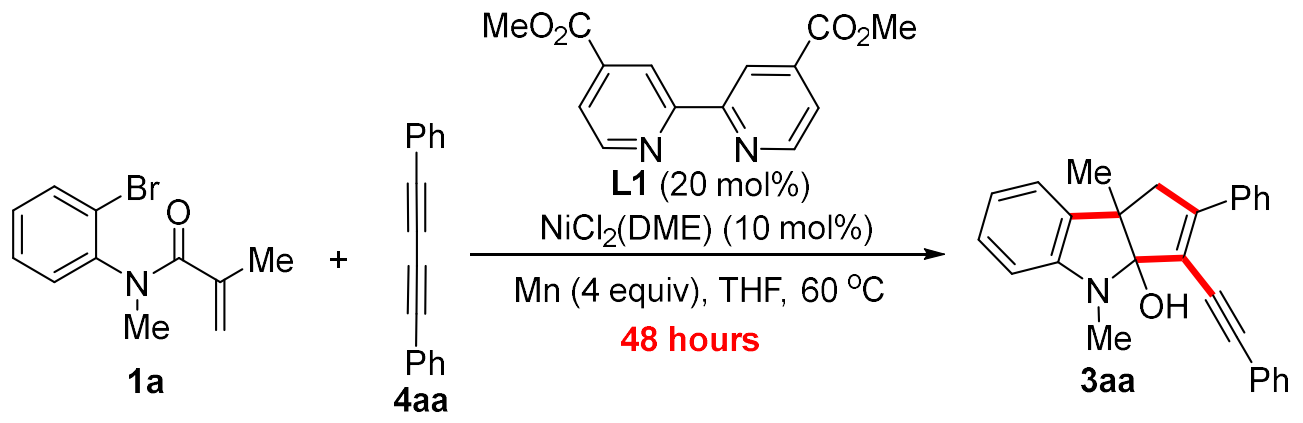

Experimental procedure: An oven-dried seal-tube equipped with a PTFE-coated stir bar was charged with $1 \mathrm{a}(0.1 \mathrm{mmol}, 25.4 \mathrm{mg})$ and $\mathbf{L} 1(0.02 \mathrm{mmol}, 5.3 \mathrm{mg})$ and brought into an argon filled glovebox. $\mathrm{NiCl}_{2}$ (DME) $(0.01 \mathrm{mmol}, 2.1 \mathrm{mg})$, 4 aa $(0.3 \mathrm{mmol}, 60.2 \mathrm{mg})$ and $\mathrm{Mn}^{0}$ (0.4 mmol, $23.4 \mathrm{mg}$ ) was added, followed by anhydrous THF $(2 \mathrm{~mL})$. The tube was sealed and removed from the glovebox. Then the reaction was stirred at room temperature for $10 \mathrm{~min}$ and heated at $60^{\circ} \mathrm{C}$ for $48 \mathrm{~h}$. The solvent was then removed under vacuum and the residue was purified by chromatography on silica gel, eluting with ethyl acetate/toluene 1:50-1:10 to afford the desired dialkyne 3aa (26.7 mg, 71\%).

Conclusion: These results clearly demonstrated that 1,3-diyne 4 is a key intermediate in this transformation. 


\subsection{Evaluation of the role of $\mathrm{MnBr}_{2}$}

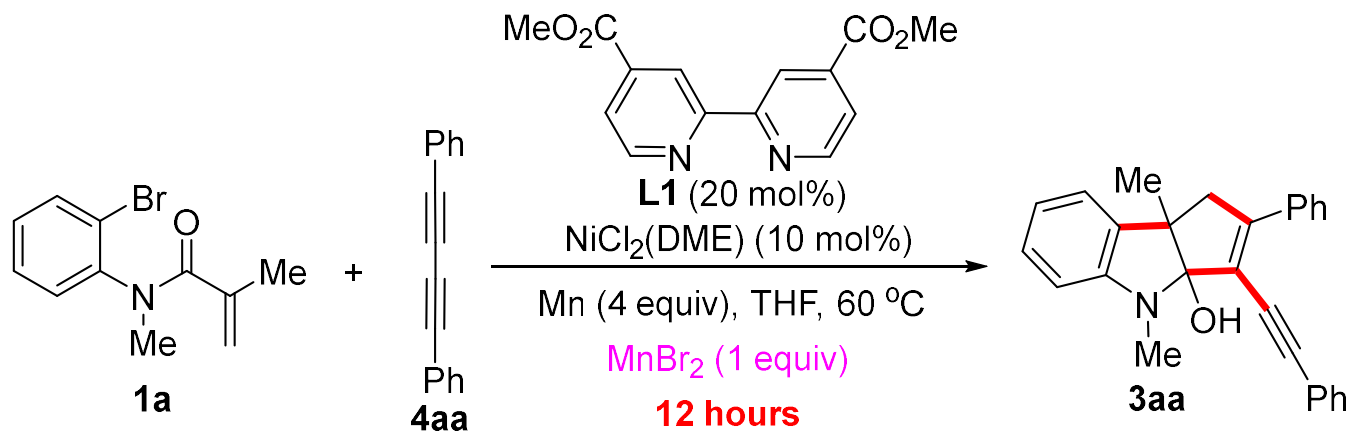

Experimental procedure: An oven-dried seal-tube equipped with a PTFE-coated stir bar was charged with 1a $(0.1 \mathrm{mmol}, 25.2 \mathrm{mg})$ and $\mathbf{L 1}(0.02 \mathrm{mmol}, 5.1 \mathrm{mg})$, and brought into an argon filled glovebox. $\mathrm{NiCl}_{2}(\mathrm{DME})(0.01 \mathrm{mmol}, 2.2 \mathrm{mg})$, 4aa $(0.3 \mathrm{mmol}, 60.0 \mathrm{mg})$, $\mathrm{MnBr}_{2}(0.1 \mathrm{mmol}, 22.0 \mathrm{mg})$ and $\mathrm{Mn}^{0}(0.4 \mathrm{mmol}, 23.3 \mathrm{mg})$ was added, followed by anhydrous THF $(2 \mathrm{~mL})$. The tube was sealed and removed from the glovebox. Then the reaction was stirred at room temperature for $10 \mathrm{~min}$ and heated at $60{ }^{\circ} \mathrm{C}$ for $12 \mathrm{~h}$. The solvent was then removed under vacuum and the residue was purified by chromatography on silica gel, eluting with ethyl acetate/toluene 1:50-1:10 to afford the desired dialkyne 3aa (26.1 mg, 70\%).

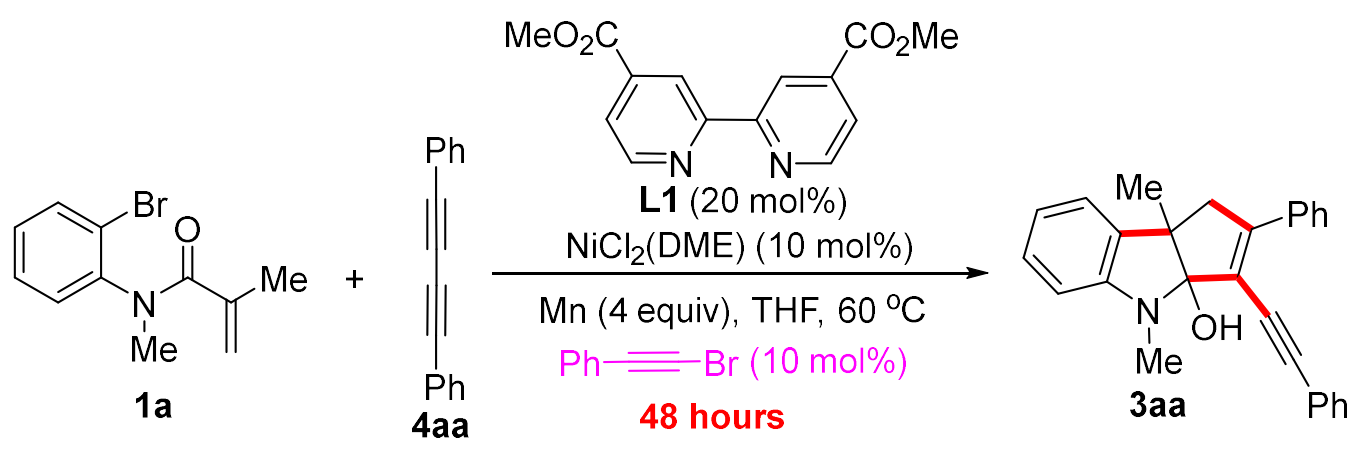

Experimental procedure: An oven-dried seal-tube equipped with a PTFE-coated stir bar was charged with $1 \mathrm{a}(0.1 \mathrm{mmol}, 25.4 \mathrm{mg})$ and $\mathbf{L} 1(0.02 \mathrm{mmol}, 5.2 \mathrm{mg})$, and brought into an argon filled glovebox. $\mathrm{NiCl}_{2}(\mathrm{DME})$ (0.01 mmol, $\left.2.2 \mathrm{mg}\right)$, 4aa (0.3 mmol, $\left.60.3 \mathrm{mg}\right), 2 \mathrm{a}$ $(0.01 \mathrm{mmol}, 2.0 \mathrm{mg})$ and $\mathrm{Mn}^{0}(0.4 \mathrm{mmol}, 23.4 \mathrm{mg})$ was added, followed by anhydrous THF (2 mL). The tube was sealed and removed from the glovebox. Then the reaction was stirred at room temperature for $10 \mathrm{~min}$ and heated at $60^{\circ} \mathrm{C}$ for $48 \mathrm{~h}$. The solvent was then removed under vacuum and the residue was purified by chromatography on silica gel, 
eluting with ethyl acetate/toluene 1:50-1:10 to afford the desired dialkyne 3aa (26.4 mg, $70 \%)$.

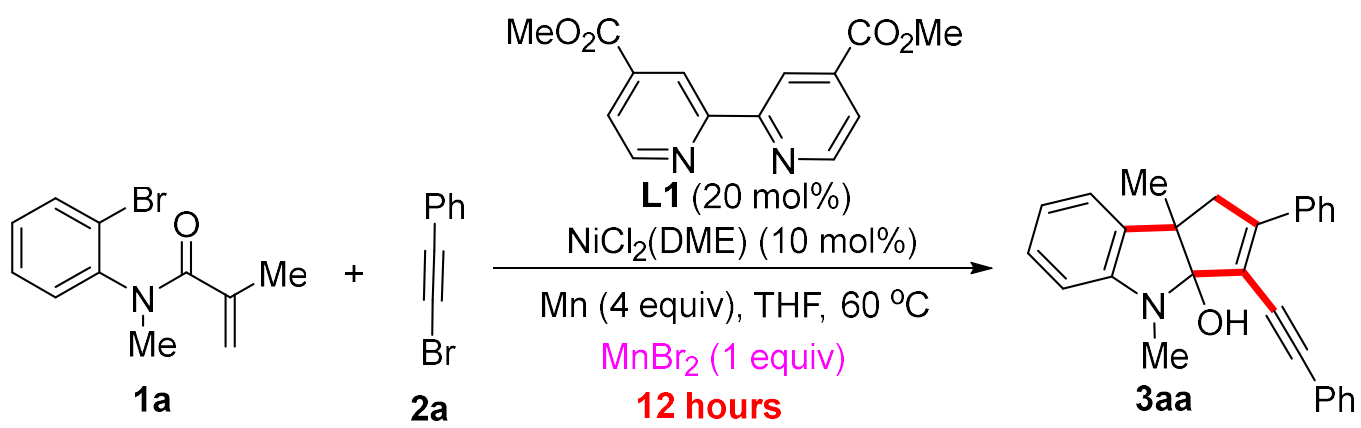

Experimental procedure: An oven-dried seal-tube equipped with a PTFE-coated stir bar was charged with $1 \mathrm{a}(0.1 \mathrm{mmol}, 25.4 \mathrm{mg})$ and $\mathbf{L} 1(0.02 \mathrm{mmol}, 5.2 \mathrm{mg})$, and brought into an argon filled glovebox. $\mathrm{NiCl}_{2}$ (DME) (0.01 mmol, $\left.2.3 \mathrm{mg}\right), 2 \mathrm{a}(0.3 \mathrm{mmol}, 54.4 \mathrm{mg}), \mathrm{MnBr}_{2}$ $(0.1 \mathrm{mmol}, 22.1 \mathrm{mg})$ and $\mathrm{Mn}^{0}(0.4 \mathrm{mmol}, 23.7 \mathrm{mg})$ was added, followed by anhydrous THF ( $2 \mathrm{~mL})$. The tube was sealed and removed from the glovebox. Then the reaction was stirred at room temperature for $10 \mathrm{~min}$ and heated at $60^{\circ} \mathrm{C}$ for $12 \mathrm{~h}$. The solvent was then removed under vacuum and the residue was purified by chromatography on silica gel, eluting with ethyl acetate/toluene 1:50-1:10 to afford the desired dialkyne 3aa (25.6 mg, $68 \%)$.

Conclusion: These results clearly demonstrated that the addition of $\mathrm{MnBr}_{2}$ does not affect the yields, but it seems to play a key role in significantly accelerating the carbonickelation cyclization process. 


\subsection{Evaluation of potential nickel intermediates Synthesis of $\sigma$-alkyl-Ni(II) complex 6}

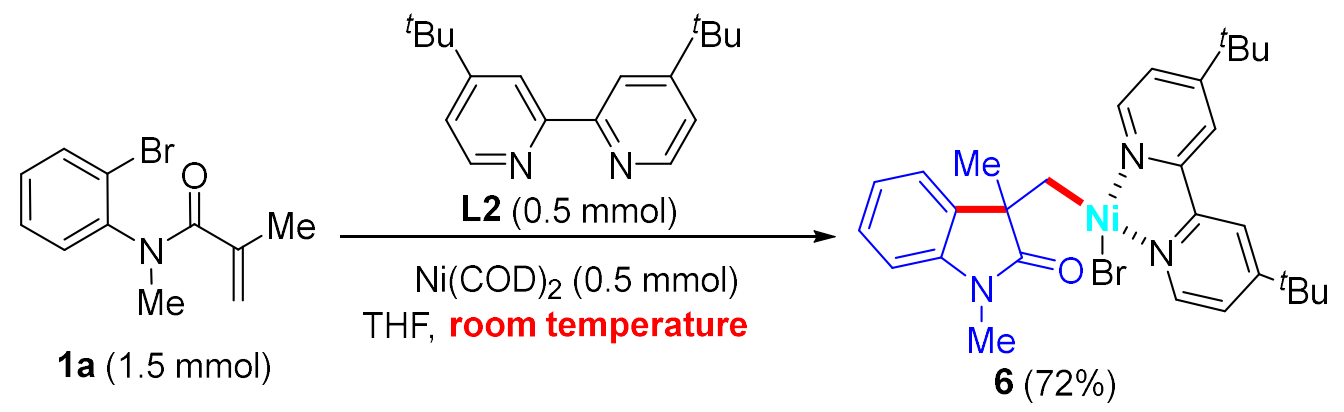

In an argon filled glove box, a $50 \mathrm{~mL}$ round bottom flask containing a PTFE-coated stirring bar was charged with $\mathrm{Ni}(\mathrm{COD})_{2}(0.5 \mathrm{mmol}, 135.5 \mathrm{mg})$, L2 $(0.5 \mathrm{mmol}, 134.3 \mathrm{mg})$ and dry THF $(4 \mathrm{~mL})$ giving a deep purple mixture which was stirred for overnight. 1a (1.5 $\mathrm{mmol}, 380.3 \mathrm{mg}$ ) was added to the purple mixture and stirred for additional 2 hours. Dry $n$-hexane $(20 \mathrm{~mL})$ was added to the red colored mixture and filtered. The participate was washed with $n$-hexane $(5 \times 10 \mathrm{~mL})$ to remove the residual cyclooctadiene and $1 \mathrm{a}$, and the resulting solid was dried under vacuum to give 6 as a pink complex $(204.2 \mathrm{mg}, 72 \%) .{ }^{1} \mathrm{H}$ NMR (400 MHz; acetone-d6) $\delta 8.55(\mathrm{~d}, J=4.3 \mathrm{~Hz}, 2 \mathrm{H}), 8.25(\mathrm{~d}, J=1.5 \mathrm{~Hz}, 2 \mathrm{H}), 7.80(\mathrm{~d}$, $J=6.9 \mathrm{~Hz}, 1 \mathrm{H}), 7.55-7.41(\mathrm{~m}, 2 \mathrm{H}), 6.97-6.89(\mathrm{~m}, 1 \mathrm{H}), 6.72(\mathrm{t}, J=7.4 \mathrm{~Hz}, 1 \mathrm{H}), 6.64(\mathrm{~d}, J$ $=7.7 \mathrm{~Hz}, 1 \mathrm{H}), 2.88(\mathrm{~s}, 3 \mathrm{H}), 1.71(\mathrm{~d}, J=8.5 \mathrm{~Hz}, 1 \mathrm{H}), 1.52(\mathrm{~s}, 3 \mathrm{H}), 1.41(\mathrm{~s}, 18 \mathrm{H}), 0.97(\mathrm{~d}$, $J=8.5 \mathrm{~Hz}, 1 \mathrm{H}$ ). The $\sigma$-alkyl-Ni (II) complex 6 was unstable. Therefore it was generated and used immediately. 


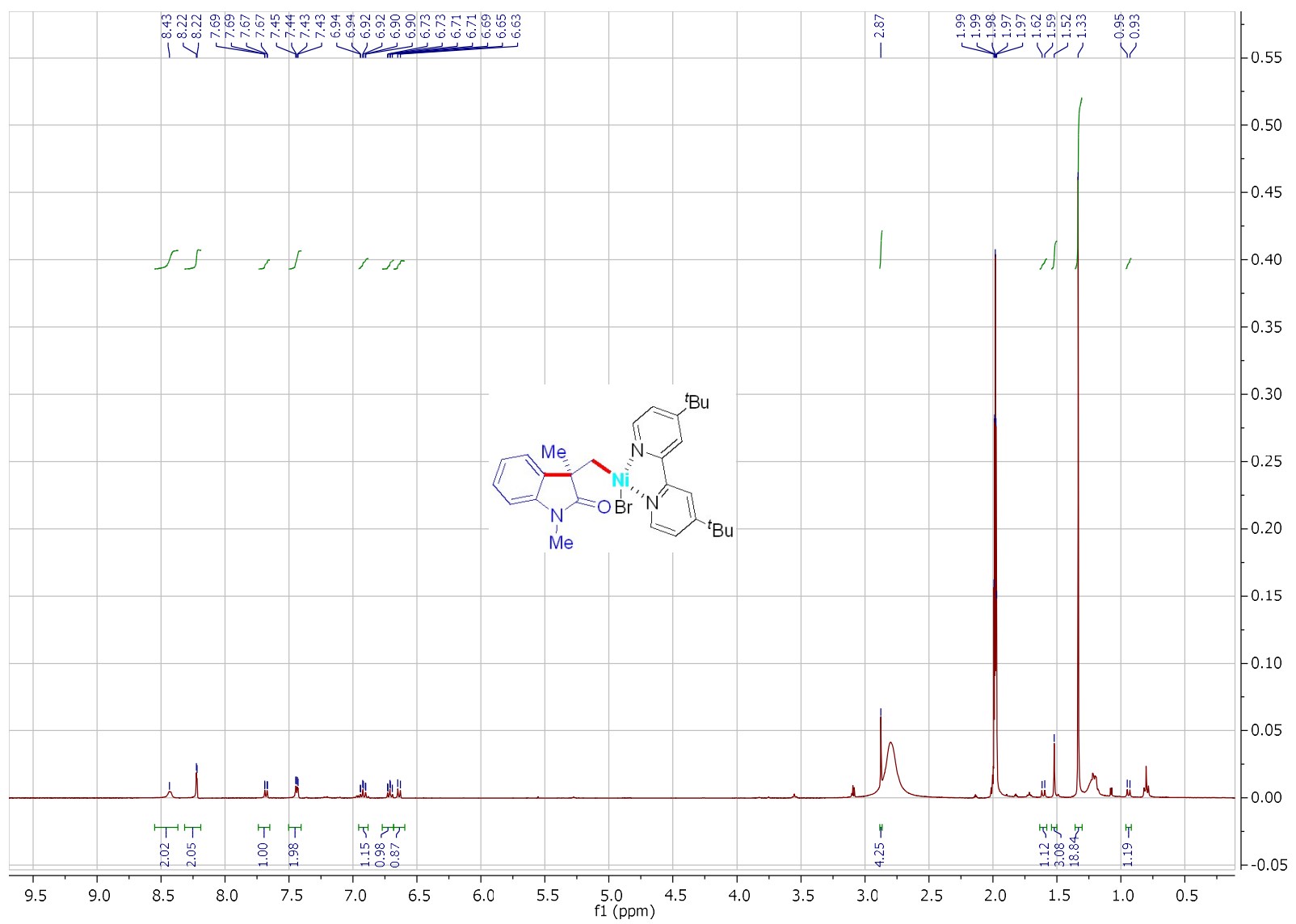


Stoichiometric experiments with complex 6 with $4 \mathrm{aa}$ in the presence of $\mathrm{MnBr}_{2}$

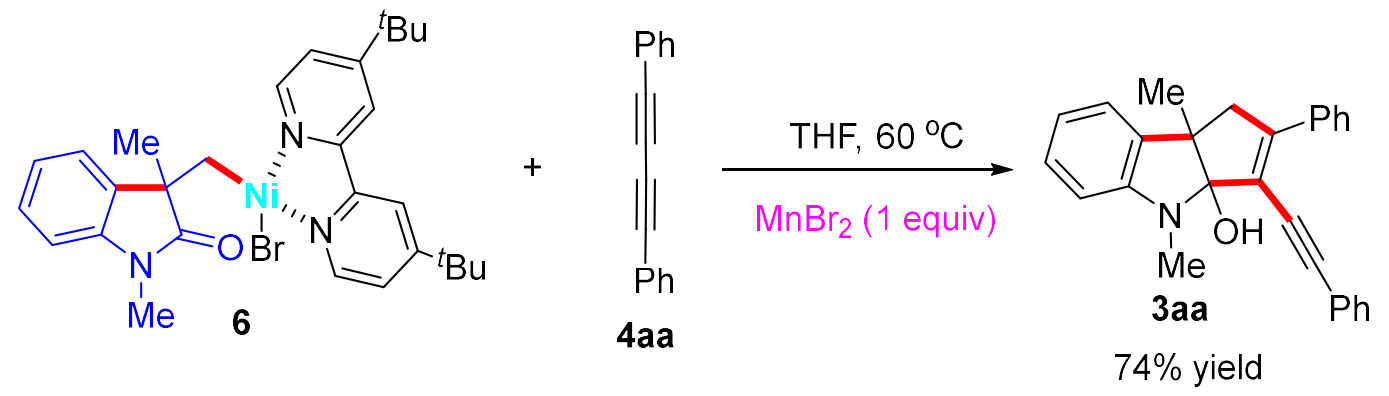

Experimental procedure: An oven-dried seal-tube equipped with a PTFE-coated stir bar was charged with 4 aa $(0.15 \mathrm{mmol}, 30.3 \mathrm{mg})$ and brought into an argon filled glovebox. 6 $(0.05 \mathrm{mmol}, 29.2 \mathrm{mg})$ and $\mathrm{MnBr}_{2}(0.05 \mathrm{mmol}, 11.0 \mathrm{mg})$ was added, followed by anhydrous THF (2 mL). The tube was sealed and removed from the glovebox. Then the reaction was heated at $60{ }^{\circ} \mathrm{C}$ for $12 \mathrm{~h}$. The solvent was then removed under vacuum and the residue was purified by chromatography on silica gel, eluting with ethyl acetate/toluene 1:50-1:10 to afford the desired dialkyne 3aa (13.8 mg, 74\%).

Conclusion: These results demonstrated that $\sigma$-alkyl-Ni(II) 6 is a potential reaction intermediate in the catalytic cycle. 
Stoichiometric experiments with complex 6 with $4 a a$ in the absence of $\mathrm{MnBr}_{2}$

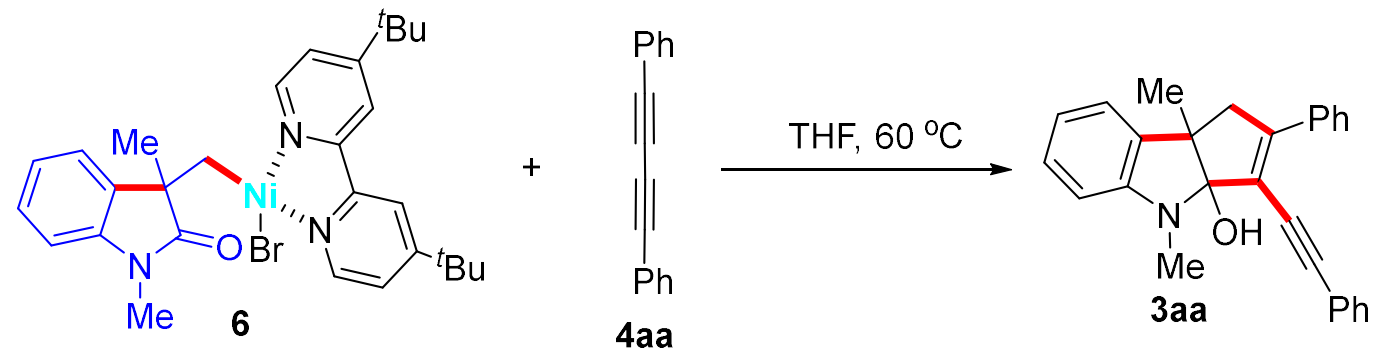

Experimental procedure: An oven-dried seal-tube equipped with a PTFE-coated stir bar was charged with $4 \mathbf{a a}(0.15 \mathrm{mmol}, 30.3 \mathrm{mg})$, and brought into an argon filled glovebox. 6 $(0.05 \mathrm{mmol}, 29.1 \mathrm{mg})$ was added, followed by anhydrous THF $(2 \mathrm{~mL})$. The tube was sealed and removed from the glovebox. Then the reaction was heated at $60{ }^{\circ} \mathrm{C}$ for $24 \mathrm{~h}$. After removal of the solvent under reduced pressure, redissolved in $\mathrm{CDCl}_{3}$ and the crude mixture analyzed by ${ }^{1} \mathrm{H}$ NMR. Only trace amount of product 3aa was observed.

Conclusion: This result confirmed that $\mathrm{MnBr}_{2}$ was indispensable for the reaction to occur. 


\subsection{Operando IR}

General ReactIR Experimental Details: For the ReactIR experiments, the reaction spectra were recorded using an IC 10 and IC 15 from Mettler-Toledo AutoChem. Data manipulation was carried out using the iC IR software, version 4.2. The reaction was carried out as follows: a three necked reaction vessel was fitted with a magnetic stirring bar in oil bath. The IR probe was inserted through an adapter into the middle neck; the other two necks were capped by septa for injections and a nitrogen line. Following evacuation under vacuum and flushing with nitrogen for three times, the three necked vessel was charged with solution of reactants. Operando IR spectra were recorded over the course of the reaction.

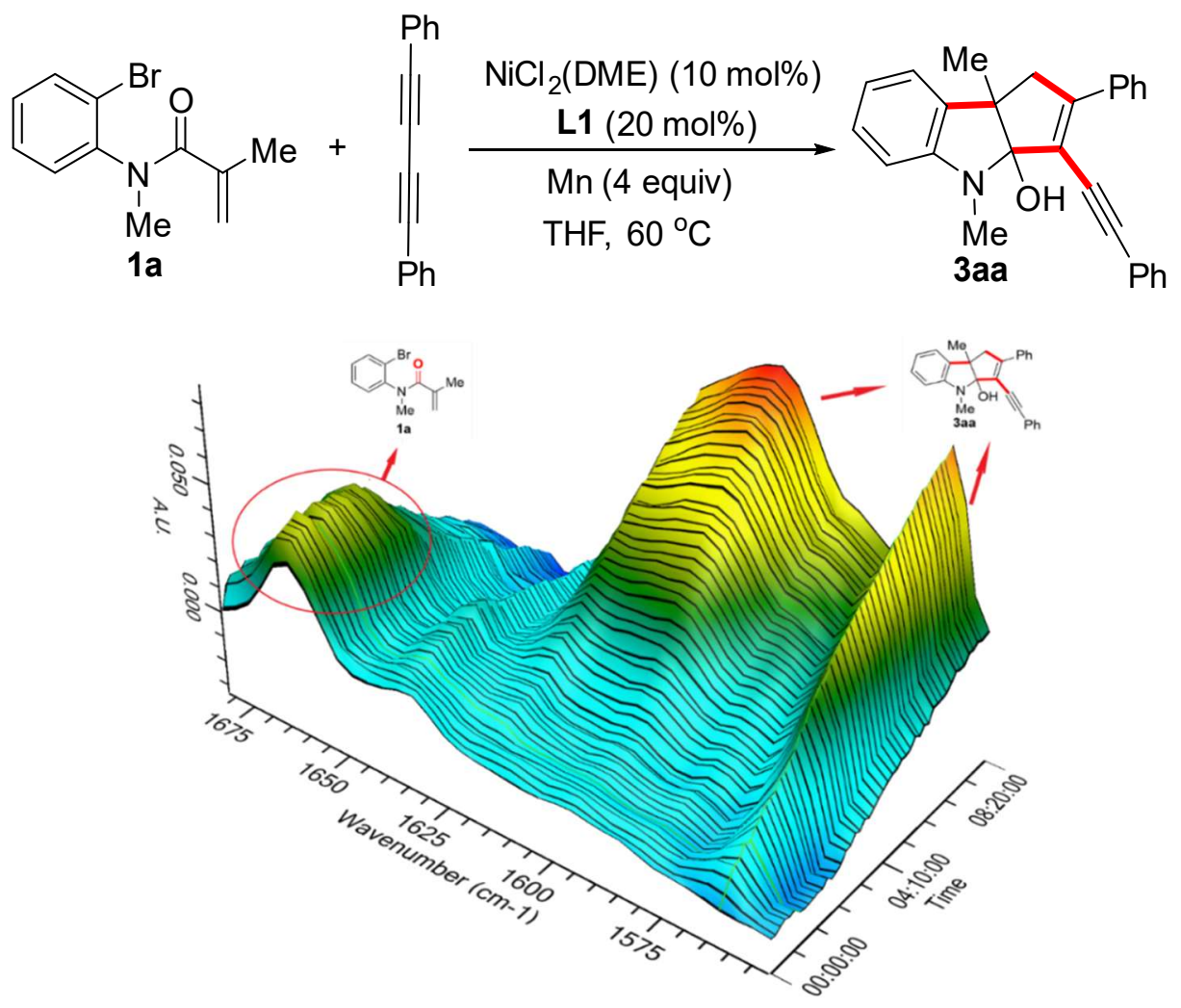

Figure S1. Kinetic profiles monitored by operando IR. 


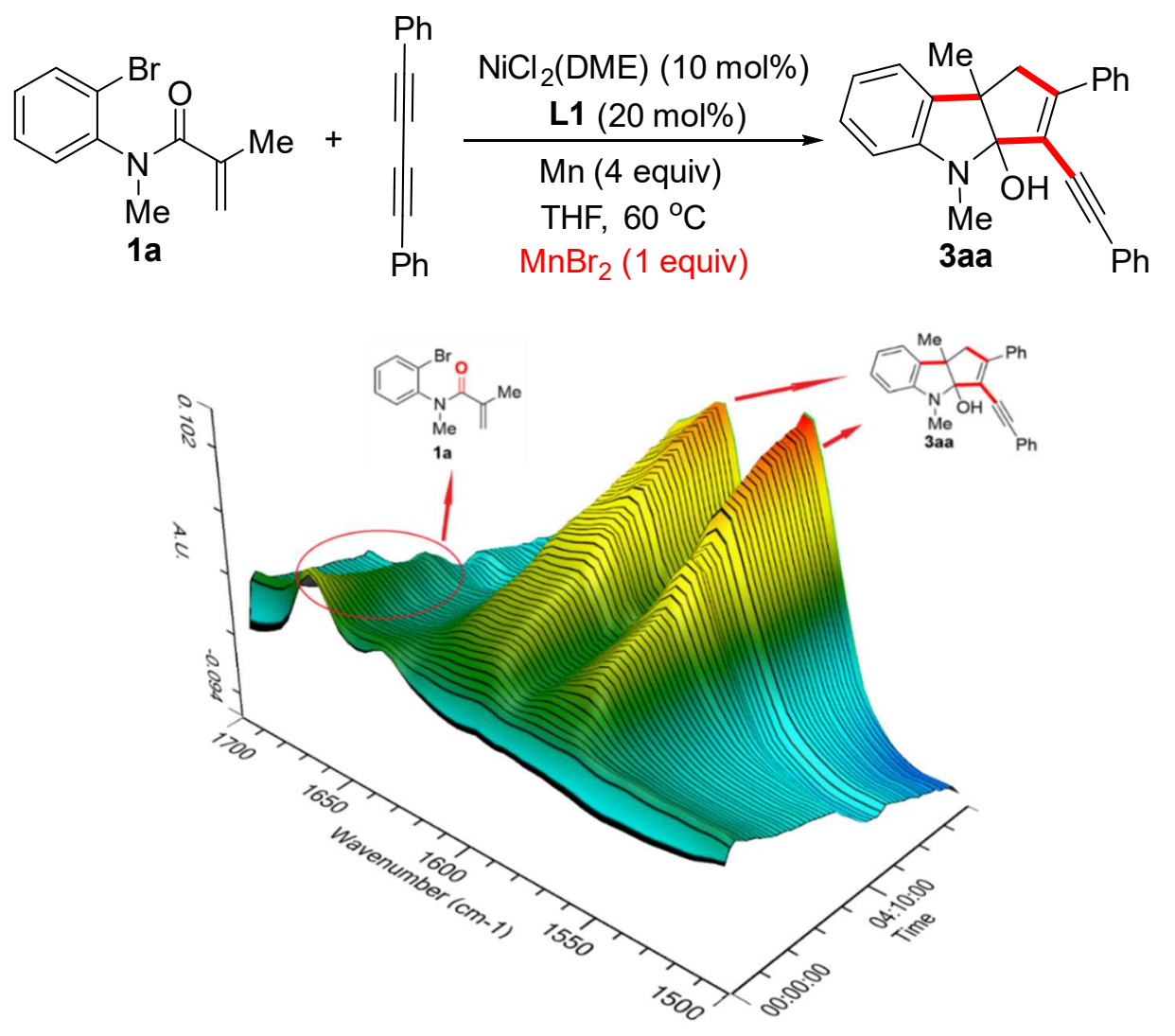

Figure S2. Kinetic profiles monitored by operando IR.

Conclusion: These results further support the hypothesis that $\mathrm{MnBr}_{2}$ acts as a Lewis acid to activate the amide to accelerate the reaction. And the step of carbonyl disappearance, that is, nucleophilic cyclization of alkenylnickel species $\mathbf{B}$ to amide, may be the ratedetermining step of the reaction. 


\section{General procedure for the synthesis of starting materials}

\subsection{Synthesis of aryl bromides $1^{2}$}
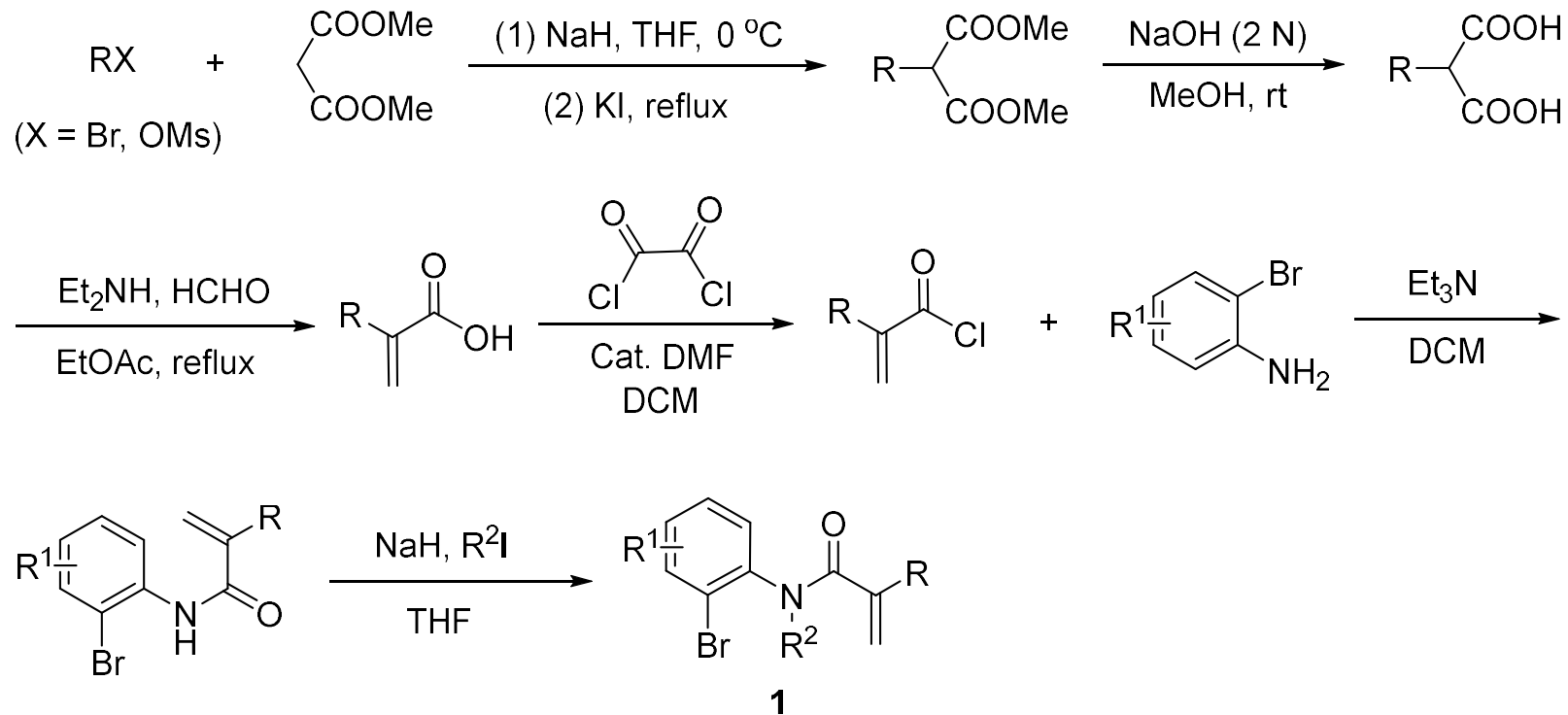

To a mixture of $\mathrm{NaH}$ (60\% dispersion in mineral oil, 1.1 equiv) and $\mathrm{KI}$ ( 0.1 equiv) in dry THF $(100 \mathrm{~mL})$ at $0{ }^{\circ} \mathrm{C}$ was added dropwise dimethyl malonate $(50 \mathrm{mmol})$. The suspension was stirred at room temperature for 30 minutes and RX (1.2 equiv) was added subsequently. The mixture was then refluxed for 16 hours. After cooling to room temperature, the resulting mixture was quenched with sat. $\mathrm{NH}_{4} \mathrm{Cl}$ solution and the aqueous phase was extracted with EtOAc. The combined organic extracts were washed with brine, dried over $\mathrm{Na}_{2} \mathrm{SO}_{4}$, filtered and concentrated under reduced pressure. The crude product was subjected to column chromatography to afford $\alpha$-alkylated dimethyl malonate.

To a solution of $\alpha$-alkylated dimethyl malonate $(30 \mathrm{mmol})$ in $\mathrm{MeOH}(50 \mathrm{~mL})$ was added $2 \mathrm{~N} \mathrm{NaOH}$ solution $(50 \mathrm{~mL})$. The resulting mixture was stirred at room temperature overnight. After removing the volatiles under reduced pressure, the aqueous layer was extracted with AcOEt $(30 \mathrm{~mL})$, acidified with $3 \mathrm{~N}$ aqueous $\mathrm{HCl}$ solutions, and extracted with EtOAc $(3 \times 40 \mathrm{~mL})$. The combined organic extracts were dried over anhydrous $\mathrm{Na}_{2} \mathrm{SO}_{4}$, filtered and concentrated to give the crude diacid, which was used directly for the next step without further purification.

To a mixture of the crude diacid (30 mmol) and paraformaldehyde (1.5 equiv) in EtOAc $(80 \mathrm{~mL})$ at $0{ }^{\circ} \mathrm{C}$ was added $\mathrm{Et}_{2} \mathrm{NH}$ (1.5 equiv). The resulting mixture was then heated at 
reflux overnight. After cooling to room temperature, the reaction mixture was acidified with $3 \mathrm{~N}$ aqueous $\mathrm{HCl}$ solution, and extracted with EtOAc $(3 \times 40 \mathrm{~mL})$. The combined organic layers were washed with brine, dried over $\mathrm{Na}_{2} \mathrm{SO}_{4}$, filtered and concentrated. The residue was purified by column chromatography on silica gel to acrylic acid for two steps.

To a mixture of the acrylic acid (30 mmol) and oxalyl dichloride (2 equiv) in DCM (30 $\mathrm{mL}$ ) at $0{ }^{\circ} \mathrm{C}$ was added DMF three drops. After stirring at room temperature for 3 hours, the mixture was concentrated and the resulting residue acryloyl chloride was used for the next step without further purification.

To a solution of substituted 2-bromoaniline $(30 \mathrm{mmol}$ ) and trimethylamine (2 equiv) in DCM $(40 \mathrm{~mL})$ at $0{ }^{\circ} \mathrm{C}$ was added dropwise a solution of acryloyl chloride (1.2 equiv) in DCM (10 mL) over $15 \mathrm{~min}$. After stirring for another $30 \mathrm{~min}$, the mixture was warmed to room temperature and stirred until the aniline was consumed completely (monitored by TLC). The resulting solution was concentrated, and the residue was dissolved in EtOAc $\left(50 \mathrm{~mL}\right.$ ) and filtered. The organic layer was washed with saturated $\mathrm{NaHCO}_{3}$ solution (50 $\mathrm{mL}$ ) and brine $(50 \mathrm{~mL})$, dried over anhydrous $\mathrm{Na}_{2} \mathrm{SO}_{4}$, filtered, and concentrated in vacuo. The residue amide was used for the next step without further purification.

To a stirred mixture of $\mathrm{NaH}$ ( $60 \%$ dispersion in mineral oil, 1.5 equiv) in dry THF (50 $\mathrm{mL}$ ) was added amide $(30 \mathrm{mmol})$ at $0{ }^{\circ} \mathrm{C}$ under $\mathrm{N}_{2}$. After being stirred at $\mathrm{rt}$ for $30 \mathrm{~min}$, the reaction was cooled to $0{ }^{\circ} \mathrm{C}$ and $\mathrm{R}^{2} \mathrm{X}$ (1.2 equiv) in dry THF $(5 \mathrm{~mL})$ was added dropwise. The mixture was then stirred at room temperature overnight. After the reaction was complete (monitored by TLC), the mixture was quenched with saturated aqueous $\mathrm{NH}_{4} \mathrm{Cl}$ at $0{ }^{\circ} \mathrm{C}$ and extracted with EtOAc $(40 \mathrm{~mL} \times 3)$. The combined extracts were washed with water and brine, dried over $\mathrm{Na}_{2} \mathrm{SO}_{4}$, filtered, and concentrated in vacuo. The residue was purified by column chromatography on silica gel to afford the aryl bromide 1 . 


\subsection{Synthesis of alkynyl bromides $2^{3}$}

$$
\mathrm{R} \rightleftharpoons \frac{\mathrm{NBS}, \mathrm{AgNO}_{3}}{\text { acetone }} \mathrm{R} \rightleftharpoons \mathrm{Br}
$$

To a solution of the alkyne (10 mmol, 1 equiv) in acetone $(20 \mathrm{~mL}, 0.5 \mathrm{M})$ was added $\mathrm{N}$-bromosuccinimide (NBS, 1.2 equiv) and $\mathrm{AgNO}_{3}$ (0.1 equiv), the resulting mixture was stirred at room temperature for $2 \mathrm{~h}$. After removal of the solvent under reduced pressure, the residue was purified by chromatography on silica gel, eluting with ethyl acetate/petroleum ether 1:50 1:10 (v/v) to afford the desired alkynyl bromides 2 .

\subsection{Synthesis of internal alkynes $7^{4}$}

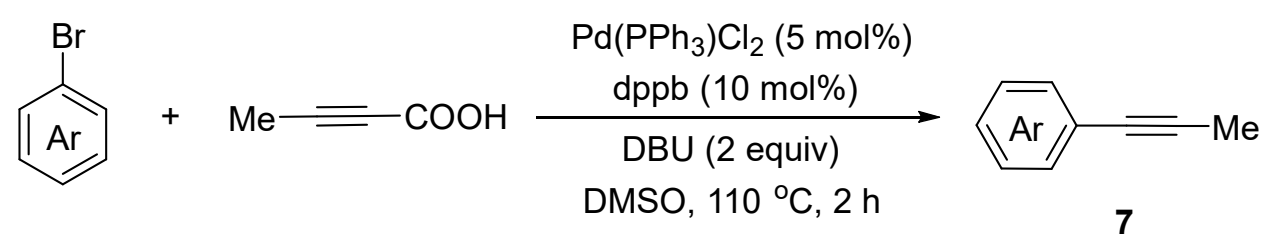

$\mathrm{Pd}\left(\mathrm{PPh}_{3}\right)_{2} \mathrm{Cl}_{2}$ (0.03 mmol), 1,4-bis(diphenylphosphino)butane $(0.06 \mathrm{mmol})$, aryl bromide $(3.0 \mathrm{mmol})$, and but-2-ynoic acid $(6.0 \mathrm{mmol})$ were combined with DBU $(6.0 \mathrm{mmol})$ in DMSO $(10.0 \mathrm{~mL})$. The resulting mixture was placed in an oil bath at $110^{\circ} \mathrm{C}$ for $2 \mathrm{~h}$. The reaction was poured into $20 \mathrm{~mL}$ of saturated aqueous ammonium chloride and extracted with $(3 \times 20 \mathrm{~mL})$ with EtOAc. The combined ether extracts were washed with brine $(60 \mathrm{~mL})$, dried over $\mathrm{MgSO}_{4}$, and filtered. After removal of the solvent under reduced pressure, the residue was purified by chromatography on silica gel, eluting with ethyl acetate/petroleum ether 1:20 1:5 (v/v) to afford the desired internal alkynes 7. 


\section{Characterization data of products}

4,8b-Dimethyl-2-phenyl-3-(phenylethynyl)-4,8b-dihydrocyclopenta[b]indol-3a(1H)-ol (3aa)

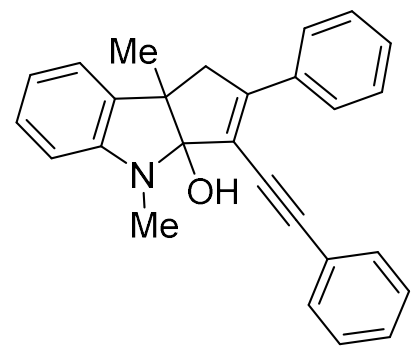

Chemical Formula: $\mathrm{C}_{27} \mathrm{H}_{23} \mathrm{NO}$

Exact Mass: 377.1780

3aa was prepared according to general procedure 2.1 using $\mathbf{1 a}$ and $\mathbf{2 a}$ and was purified by silica gel column chromatography (toluene/EtOAc $=40 / 1 \sim 5 / 1$ ) to obtain 3aa as yellow oil (72\% yield). ${ }^{1} \mathrm{H}$ NMR (400 MHz, $\left.\mathrm{CDCl}_{3}\right) \delta$ 7.93-7.84 (m, 2H), 7.55-7.46 (m, 2H), 7.43$7.29(\mathrm{~m}, 6 \mathrm{H}), 7.17-7.08(\mathrm{~m}, 2 \mathrm{H}), 6.71(\mathrm{td}, J=7.4,1.0 \mathrm{~Hz}, 1 \mathrm{H}), 6.42(\mathrm{~d}, J=7.5 \mathrm{~Hz}, 1 \mathrm{H})$, $3.20(\mathrm{~s}, 3 \mathrm{H}), 3.18(\mathrm{~d}, J=17.1 \mathrm{~Hz}, 1 \mathrm{H}), 3.09(\mathrm{~d}, J=17.1 \mathrm{~Hz}, 1 \mathrm{H}), 2.55$ (bs, 1H), 1.46 (s, $3 \mathrm{H}) ;{ }^{13} \mathrm{C}$ NMR $\left(101 \mathrm{MHz}, \mathrm{CDCl}_{3}\right) \delta 148.4,135.3,135.2,131.7,131.4,128.8,128.50$, 128.47, 128.19, 128.16, 127.5, 123.3, 122.5, 118.5, 117.7, 105.5, 97.1, 86.4, 51.9, 48.7, 29.0, 21.1; HRMS: (ESI) calcd for $\mathrm{C}_{27} \mathrm{H}_{23} \mathrm{NNaO}^{+}[\mathrm{M}+\mathrm{Na}]^{+} 400.1672$; found 400.1675 .

The enantiomeric purity was established by HPLC analysis using a chiral column: OD-H column, $30{ }^{\circ} \mathrm{C}, n$-Hexane/i-Propanol $=90 / 10$ as eluent, $254 \mathrm{~nm}, 1 \mathrm{~mL} / \mathrm{min}$. tR $=8.4 \mathrm{~min}$ (minor), 10.5 min (major).

Optical Rotation: $[\alpha]_{D^{27}}-39.4$ (c 0.4 , $\left.{ }^{i} \mathrm{PrOH}\right)$ for $82 \%$ ee. 


\section{LabSolutions 分析报告}

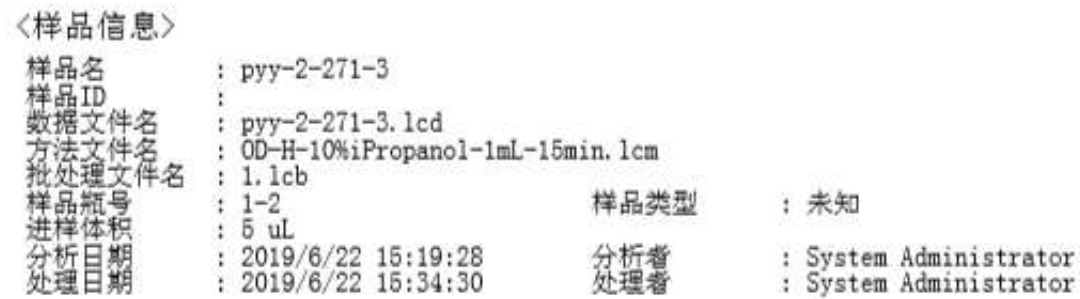

〈色谱圈〉

$\mathrm{mAU}$
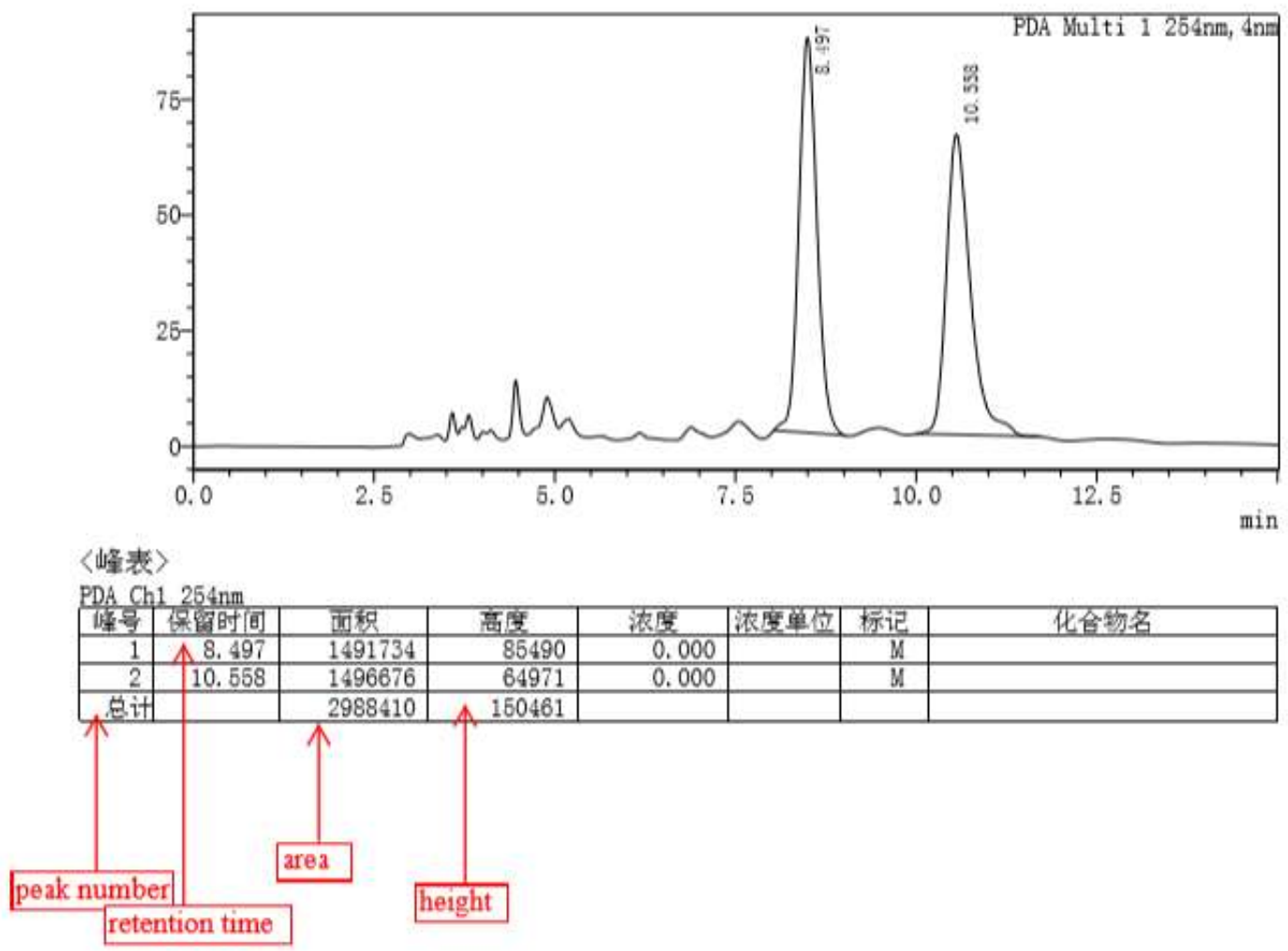


\section{LabSolutions 分析报告}

〈样品信息〉

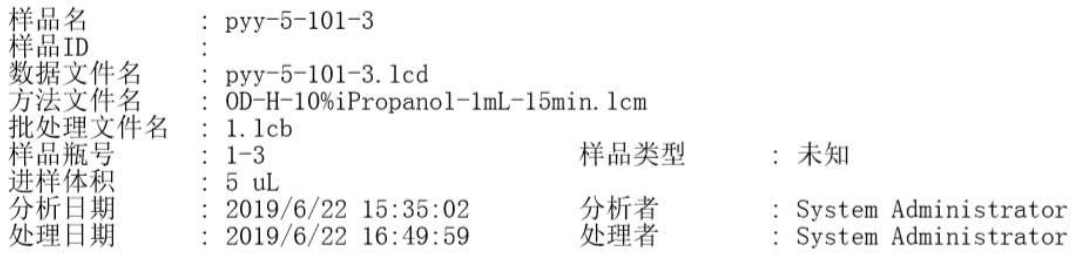

〈色谱图〉

$\mathrm{mAU}$

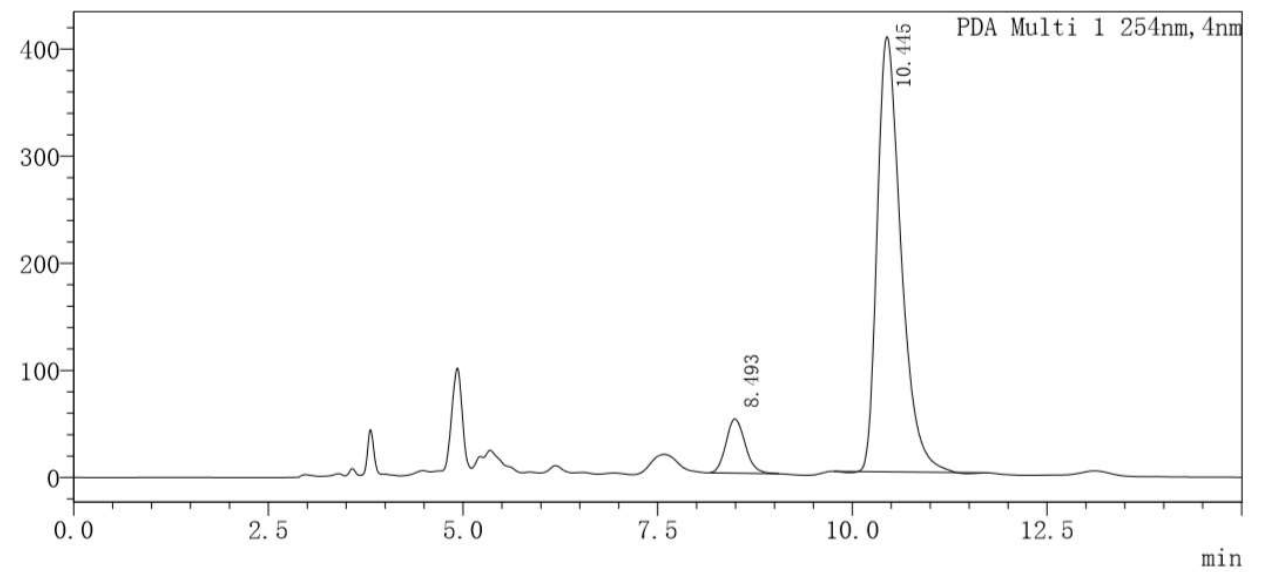

〈峰表〉

PDA Ch1 $254 \mathrm{~nm}$

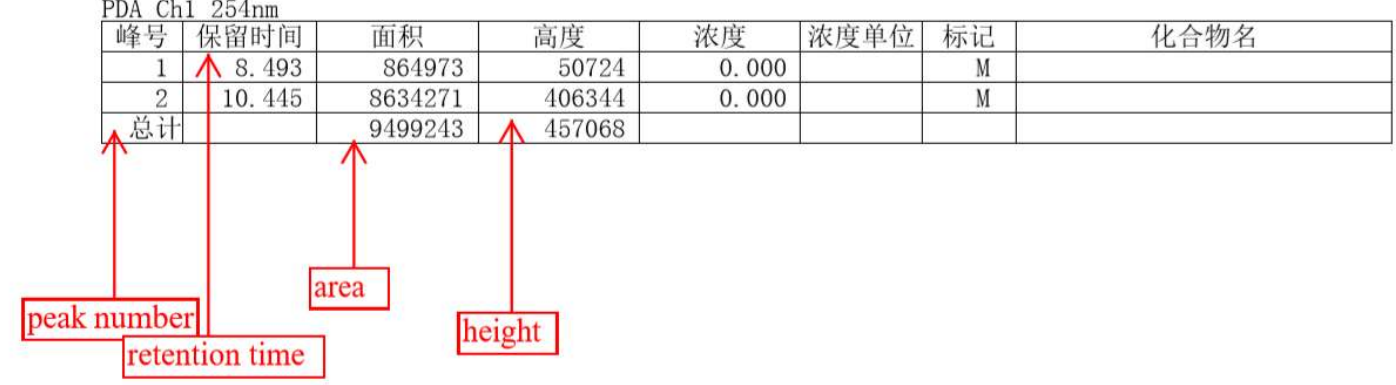


7-Fluoro-4,8b-dimethyl-2-phenyl-3-(phenylethynyl)-4,8b-dihydrocyclopenta[b]indol$3 a(1 H)$-ol (3ba)

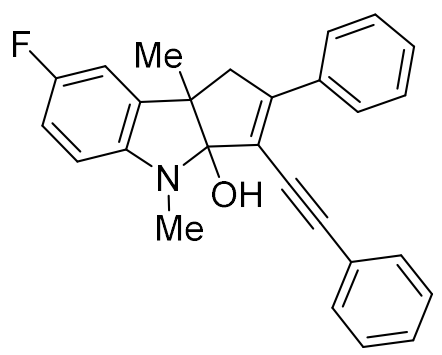

Chemical Formula: $\mathrm{C}_{27} \mathrm{H}_{22} \mathrm{FNO}$

Exact Mass: 395.1685

3ba was prepared according to general procedure 2.1 using $\mathbf{1 b}$ and $\mathbf{2 a}$ and was purified by silica gel column chromatography (toluene/EtOAc $=40 / 1 \sim 5 / 1$ ) to obtain 3 ba as yellow oil (73\% yield). ${ }^{1} \mathrm{H}$ NMR $\left(400 \mathrm{MHz}, \mathrm{CDCl}_{3}\right) \delta 7.87(\mathrm{~d}, J=7.7 \mathrm{~Hz}, 2 \mathrm{H}), 7.55-7.45(\mathrm{~m}, 2 \mathrm{H})$, 7.43-7.29 (m, 6H), 6.85 (dd, $J=8.3,2.6 \mathrm{~Hz}, 1 \mathrm{H}), 6.80(\mathrm{td}, J=8.9,2.7 \mathrm{~Hz}, 1 \mathrm{H}), 6.33-6.24$ (m, 1H), $3.15(\mathrm{~s}, 3 \mathrm{H}), 3.14(\mathrm{~d}, J=17.0 \mathrm{~Hz}, 1 \mathrm{H}), 3.08(\mathrm{~d}, J=17.0 \mathrm{~Hz}, 1 \mathrm{H}), 2.55(\mathrm{bs}, 1 \mathrm{H})$, $1.44(\mathrm{~s}, 3 \mathrm{H}) ;{ }^{13} \mathrm{C}$ NMR $\left(101 \mathrm{MHz}, \mathrm{CDCl}_{3}\right) \delta 157.6,155.3,144.6,135.1,131.4,128.9$, 128.6, 128.5, 128.2, 127.4, 123.2, 118.4, 113.9, 113.7, 110.4 (d, J = 23.9 Hz), 105.5 (d, J $=7.5 \mathrm{~Hz}$ ), 97.3, 86.2, 52.0 (d, $J=1.7 \mathrm{~Hz}), 48.4,29.4,20.8$; ${ }^{19} \mathrm{~F} \mathrm{NMR}\left(376 \mathrm{MHz}, \mathrm{CDCl}_{3}\right)$ : $\delta$-127.2; HRMS: (ESI) calcd for $\mathrm{C}_{27} \mathrm{H}_{22} \mathrm{FNNaO}+[\mathrm{M}+\mathrm{Na}]^{+} 418.1578$; found 418.1574.

4,8b-Dimethyl-2-phenyl-3-(phenylethynyl)-7-(trifluoromethyl)-4,8b-dihydrocyclopenta $[b]$ indol-3a(1H)-ol (3ca)

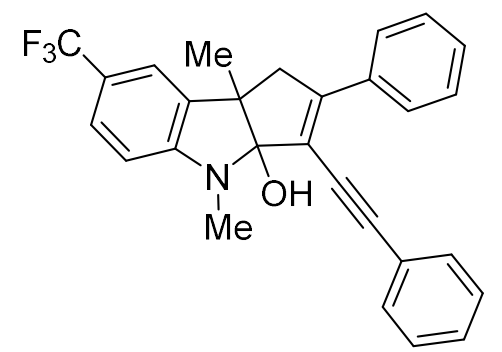

Chemical Formula: $\mathrm{C}_{28} \mathrm{H}_{22} \mathrm{~F}_{3} \mathrm{NO}$

Exact Mass: 445.1653

3ca was prepared according to general procedure 2.1 using $\mathbf{1 c}$ and $\mathbf{2 a}$ and was purified by silica gel column chromatography (toluene/EtOAc $=40 / 1 \sim 5 / 1$ ) to obtain $3 \mathrm{ca}$ as yellow 
oil $\left(72 \%\right.$ yield). ${ }^{1} \mathrm{H}$ NMR $\left(400 \mathrm{MHz}, \mathrm{CDCl}_{3}\right) \delta 7.90(\mathrm{~d}, J=7.7 \mathrm{~Hz}, 2 \mathrm{H}), 7.58-7.49(\mathrm{~m}, 2 \mathrm{H})$, 7.46-7.31 (m, 8H), $6.42(\mathrm{~d}, J=8.2 \mathrm{~Hz}, 1 \mathrm{H}), 3.24(\mathrm{~s}, 3 \mathrm{H}), 3.19(\mathrm{~d}, J=17.2 \mathrm{~Hz}, 1 \mathrm{H}), 3.13$ (d, $J=17.2 \mathrm{~Hz}, 1 \mathrm{H}$ ), 2.74 (bs, 1H), 1.49 (s, 3H); $\left.{ }^{13} \mathrm{C} \mathrm{NMR} \mathrm{(101} \mathrm{MHz,} \mathrm{CDCl}_{3}\right) \delta 150.9$, 148.6, 135.6, 134.9, 131.4, 129.1, 128.8, 128.6, 128.3, 127.5, 126.3 (q, J = 3.9 Hz), 125.2 (q, $J=270.5 \mathrm{~Hz}$ ), 123.1, 119.6 (q, $J=3.5 \mathrm{~Hz}), 119.2(\mathrm{~d}, J=32.1 \mathrm{~Hz}), 118.2,107.6,104.5$, 97.6, 85.9, 51.7, 48.9, 28.8, 21.0; $\left.{ }^{19} \mathrm{~F} \mathrm{NMR} \mathrm{(376} \mathrm{MHz,} \mathrm{CDCl}_{3}\right): \delta$-60.3; HRMS: (ESI) calcd for $\mathrm{C}_{28} \mathrm{H}_{22} \mathrm{~F}_{3} \mathrm{NNaO}^{+}[\mathrm{M}+\mathrm{Na}]^{+} 468.1546$; found 468.1552 .

7-Methoxy-4,8b-dimethyl-2-phenyl-3-(phenylethynyl)-4,8b-dihydrocyclopenta[b]indol3a(1H)-ol (3da)

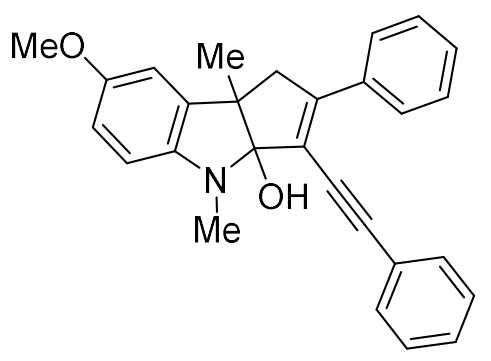

Chemical Formula: $\mathrm{C}_{28} \mathrm{H}_{25} \mathrm{NO}_{2}$

Exact Mass: 407.1885

3da was prepared according to general procedure 2.1 using $\mathbf{1 d}$ and $\mathbf{2 a}$ and was purified by silica gel column chromatography (toluene/EtOAc $=40 / 1 \sim 5 / 1$ ) to obtain 3da as yellow oil (62\% yield). ${ }^{1} \mathrm{H}$ NMR (400 MHz, $\left.\mathrm{CDCl}_{3}\right) \delta$ 7.91-7.83 (m, 2H), 7.52-7.45 (m, 2H), 7.42$7.30(\mathrm{~m}, 6 \mathrm{H}), 6.77(\mathrm{~d}, J=2.6 \mathrm{~Hz}, 1 \mathrm{H}), 6.68(\mathrm{dd}, J=8.4,2.6 \mathrm{~Hz}, 1 \mathrm{H}), 6.33(\mathrm{~d}, J=8.4 \mathrm{~Hz}$, 1H), $3.76(\mathrm{~s}, 3 \mathrm{H}), 3.17$ (d, J = $17.1 \mathrm{~Hz}, 1 \mathrm{H}), 3.15(\mathrm{~s}, 3 \mathrm{H}), 3.08$ (d, J=17.1 Hz, 1H), 1.62 (bs, $1 \mathrm{H}), 1.43(\mathrm{~s}, 3 \mathrm{H}) ;{ }^{13} \mathrm{C}$ NMR $\left(101 \mathrm{MHz}, \mathrm{CDCl}_{3}\right) \delta$ 152.8, 148.4, 142.8, 136.7, 135.3, $131.9,131.4,128.8,128.48,128.46,128.1,127.5,123.4,118.5,112.1,110.6,105.8,97.1$, 86.5, 56.1 , 52.0, 48.3, 29.7, 20.9; HRMS: (ESI) calcd for $\mathrm{C}_{28} \mathrm{H}_{26} \mathrm{NO}_{2}+[\mathrm{M}+\mathrm{H}]^{+} 408.1958$; found 408.1952 .

4,6,8b-Trimethyl-2-phenyl-3-(phenylethynyl)-4,8b-dihydrocyclopenta[b]indol-3a(1H)-ol (3fa) 


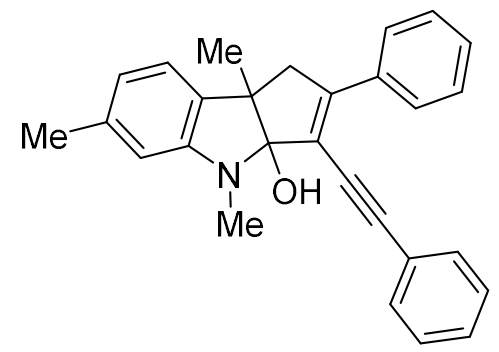

Chemical Formula: $\mathrm{C}_{28} \mathrm{H}_{25} \mathrm{NO}$

Exact Mass: 391.1936

3fa was prepared according to general procedure 2.1 using $\mathbf{1 f}$ and $\mathbf{2 a}$ and was purified by silica gel column chromatography (toluene/EtOAc $=40 / 1 \sim 5 / 1$ ) to obtain 3 fa as yellow oil (67\% yield). ${ }^{1} \mathrm{H}$ NMR (400 MHz, $\left.\mathrm{CDCl}_{3}\right)$ ס 7.89-7.82 (m, 2H), 7.53-7.46 (m, 2H), 7.41$7.28(\mathrm{~m}, 6 \mathrm{H}), 7.00(\mathrm{~d}, J=7.4 \mathrm{~Hz}, 1 \mathrm{H}), 6.53(\mathrm{~d}, J=7.6 \mathrm{~Hz}, 1 \mathrm{H}), 6.25(\mathrm{~s}, 1 \mathrm{H}), 3.17(\mathrm{~s}, 3 \mathrm{H})$, $3.16(\mathrm{~d}, J=15.2 \mathrm{~Hz}, 1 \mathrm{H}$ ), 3.07 (d, $J=17.4 \mathrm{~Hz}, 1 \mathrm{H}), 2.48$ (bs, 1H), 2.31 (s, 3H), 1.43 (s, $3 \mathrm{H}) ;{ }^{13} \mathrm{C}$ NMR $\left(101 \mathrm{MHz}, \mathrm{CDCl}_{3}\right) \delta 148.5,138.2,135.3,132.5,131.4,128.8,128.49$, 128.47, 128.1, 127.5, 123.4, 122.2, 118.5, 118.2, 106.5, 97.1, 86.5, 51.5, 48.7, 29.0, 21.9, 21.2; HRMS: (ESI) calcd for $\mathrm{C}_{28} \mathrm{H}_{25} \mathrm{NNaO}^{+}[\mathrm{M}+\mathrm{Na}]^{+} 414.1828$; found 414.1817.

6-Methoxy-4,8b-dimethyl-2-phenyl-3-(phenylethynyl)-4,8b-dihydrocyclopenta[b]indol$3 a(1 H)$-ol (3ga)

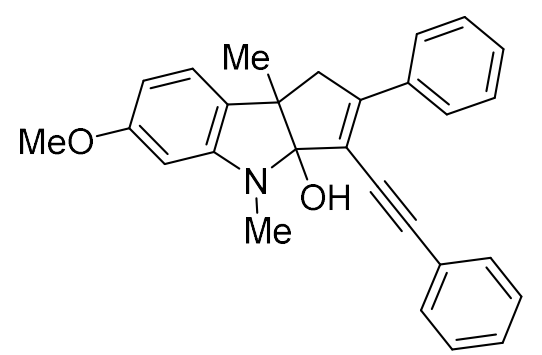

Chemical Formula: $\mathrm{C}_{28} \mathrm{H}_{25} \mathrm{NO}_{2}$

Exact Mass: 407.1885

3ga was prepared according to general procedure 2.1 using $\mathbf{1 g}$ and $\mathbf{2 a}$ and was purified by silica gel column chromatography (toluene/EtOAc $=40 / 1 \sim 5 / 1$ ) to obtain $3 \mathrm{ga}$ as yellow oil (70\% yield). ${ }^{1} \mathrm{H}$ NMR (400 MHz, $\left.\mathrm{CDCl}_{3}\right) \delta$ 7.97-7.85 (m, 2H), 7.59-7.48 (m, $\left.2 \mathrm{H}\right), 7.46-$ $7.25(\mathrm{~m}, 6 \mathrm{H}), 7.02(\mathrm{~d}, J=8.0 \mathrm{~Hz}, 1 \mathrm{H}), 6.25$ (dd, J = 8.0, $2.3 \mathrm{~Hz}, 1 \mathrm{H}), 6.04(\mathrm{~d}, J=2.3 \mathrm{~Hz}$, 1H), $3.80(\mathrm{~s}, 3 \mathrm{H}), 3.20(\mathrm{~s}, 3 \mathrm{H}), 3.17(\mathrm{~d}, J=17.2 \mathrm{~Hz}, 1 \mathrm{H}), 3.08(\mathrm{~d}, J=17.2 \mathrm{~Hz}, 1 \mathrm{H}), 2.69$ 
(bs, 1H), 1.44 (s, 3H); ${ }^{13} \mathrm{C}$ NMR (101 MHz, $\left.\mathrm{CDCl}_{3}\right) \delta$ 160.7, 149.6, 135.3, 131.4, 129.1, 128.8, 128.53, 128.50, 128.3, 128.2, 127.9, 127.5, 123.4, 122.8, 118.5, 101.4, 97.1, 93.2, 86.5, 55.4, 51.2, 48.8, 29.0, 21.4; HRMS: (ESI) calcd for $\mathrm{C}_{28} \mathrm{H}_{25} \mathrm{NNaO}_{2}+[\mathrm{M}+\mathrm{Na}]^{+} 430.1778$; found 430.1783 .

6-Chloro-4,8b-dimethyl-2-phenyl-3-(phenylethynyl)-4,8b-dihydrocyclopenta[b]indol$3 \mathrm{a}(1 H)$-ol (3ha)

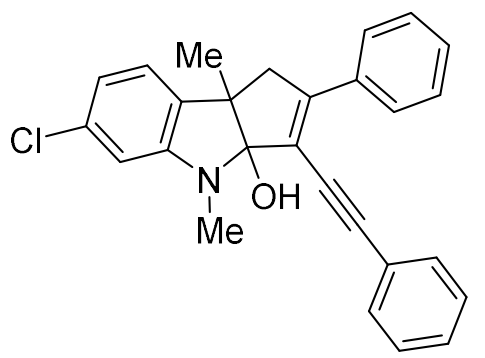

Chemical Formula: $\mathrm{C}_{27} \mathrm{H}_{22} \mathrm{CINO}$

Exact Mass: 411.1390

3ha was prepared according to general procedure 2.1 using $\mathbf{1 h}$ and $2 \mathrm{a}$ and was purified by silica gel column chromatography (toluene/EtOAc $=40 / 1 \sim 5 / 1$ ) to obtain 3ha as yellow oil (69\% yield). ${ }^{1} \mathrm{H}$ NMR $\left(400 \mathrm{MHz}, \mathrm{CDCl}_{3}\right) \delta 7.86(\mathrm{~d}, J=7.7 \mathrm{~Hz}, 2 \mathrm{H}), 7.50(\mathrm{~d}, J=3.6 \mathrm{~Hz}$, 2H), 7.43-7.29 (m, 6H), $6.99(\mathrm{~d}, J=7.7 \mathrm{~Hz}, 1 \mathrm{H}), 6.65(\mathrm{~d}, J=7.3 \mathrm{~Hz}, 1 \mathrm{H}), 6.36(\mathrm{~s}, 1 \mathrm{H})$, $3.16(\mathrm{~s}, 3 \mathrm{H}), 3.13$ (d, J = 17.2 Hz, 1H), 3.08 (d, $J=17.1 \mathrm{~Hz}, 1 \mathrm{H}$ ), 2.57 (bs, 1H), 1.43 (s, $3 \mathrm{H}) ;{ }^{13} \mathrm{C} N M R\left(101 \mathrm{MHz}, \mathrm{CDCl}_{3}\right) \delta 149.5,148.5,135.1,133.9,133.8,131.4,129.0,128.7$, 128.5, 128.2, 127.4, 123.2, 123.1, 118.3, 117.2, 107.6, 105.8, 97.4, 86.0, 51.5, 48.6, 28.9, 21.0; HRMS: (ESI) calcd for $\mathrm{C}_{27} \mathrm{H}_{22} \mathrm{CINNaO}^{+}[\mathrm{M}+\mathrm{Na}]^{+} 434.1282$; found 434.1282 .

4,5,8b-trimethyl-2-phenyl-3-(phenylethynyl)-4,8b-dihydrocyclopenta[ $b]$ indol-3a( $(1 H)$-ol (3ia) 


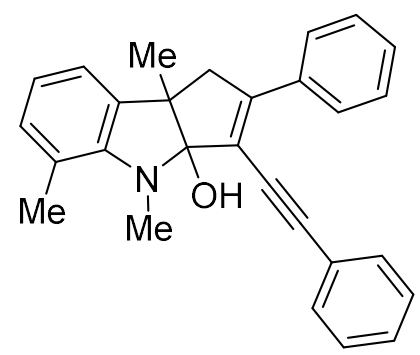

Chemical Formula: $\mathrm{C}_{28} \mathrm{H}_{25} \mathrm{NO}$

Exact Mass: 391.1936

3ia was prepared according to general procedure 2.1 using $1 \mathbf{i}$ and $2 \mathrm{a}$ and was purified by silica gel column chromatography (toluene/EtOAc $=40 / 1 \sim 5 / 1$ ) to obtain 3ia as yellow oil (57\% yield). ${ }^{1} \mathrm{H}$ NMR $\left(400 \mathrm{MHz}, \mathrm{CDCl}_{3}\right) \delta 7.89(\mathrm{~d}, J=7.2 \mathrm{~Hz}, 2 \mathrm{H}), 7.57-7.46(\mathrm{~m}, 2 \mathrm{H})$, 7.45-7.29 (m, 6H), 7.02-6.96 (m, 1H), $6.87(\mathrm{~d}, J=7.5 \mathrm{~Hz}, 1 \mathrm{H}), 6.65(\mathrm{t}, J=7.4 \mathrm{~Hz}, 1 \mathrm{H})$, $3.50(\mathrm{~s}, 3 \mathrm{H}), 3.14(\mathrm{~d}, J=17.1 \mathrm{~Hz}, 1 \mathrm{H}), 3.06(\mathrm{~d}, J=17.1 \mathrm{~Hz}, 1 \mathrm{H}), 2.57$ (bs, 1H), 2.50 (s, $3 \mathrm{H}), 1.44$ (s, 3H); ${ }^{13} \mathrm{C}$ NMR $\left(101 \mathrm{MHz}, \mathrm{CDCl}_{3}\right) \delta$ 148.8, 146.4, 135.8, 135.4, 131.9, 131.4, 128.8, 128.5, 128.2, 127.5, 123.4, 120.6, 118.9, 118.2, 117.8, 97.0, 86.7, 51.6, 49.2, 32.6, 21.2, 19.8; HRMS: (ESI) calcd for $\mathrm{C}_{28} \mathrm{H}_{25} \mathrm{NNaO}^{+}[\mathrm{M}+\mathrm{Na}]^{+}$414.1828; found 414.1925.

4,10b-dimethyl-2-phenyl-3-(phenylethynyl)-4,10b-dihydrobenzo[f]cyclopenta[b]indol$3 a(1 H)-o l(3 j a)$

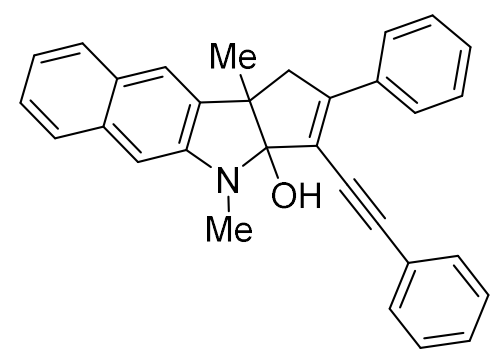

Chemical Formula: $\mathrm{C}_{31} \mathrm{H}_{25} \mathrm{NO}$

Exact Mass: 427.1936

3ja was prepared according to general procedure 2.1 using $3-(N$ methylmethacrylamido)naphthalen-2-yl trifluoromethanesulfonate and $2 \mathrm{a}$ and was purified by silica gel column chromatography (toluene/EtOAc $=40 / 1 \sim 5 / 1$ ) to obtain $3 \mathrm{ja}$ as yellow oil (63\% yield). ${ }^{1} \mathrm{H}$ NMR (400 MHz, $\left.\mathrm{CDCl}_{3}\right) \delta$ 7.96-7.87 (m, 2H), 7.74-7.64 (m, 2H), 7.60-7.53 (m, 3H), 7.47-7.33 (m, 6H), 7.26-7.18 (m, 2H), $6.63(\mathrm{~s}, 1 \mathrm{H}), 3.34(\mathrm{~s}, 3 \mathrm{H}), 3.30$ 
(d, $J=17.2 \mathrm{~Hz}, 1 \mathrm{H}), 3.21(\mathrm{~d}, J=17.2 \mathrm{~Hz}, 1 \mathrm{H}), 2.68(\mathrm{~s}, 1 \mathrm{H}), 1.58(\mathrm{~s}, 3 \mathrm{H}) ;{ }^{13} \mathrm{C}$ NMR $(101$ $\left.\mathrm{MHz}, \mathrm{CDCl}_{3}\right) \delta 148.2,147.1,138.8,135.22,135.16,131.4,128.9,128.6,128.5,128.4$, 128.2, 127.6, 127.4, 125.9, 125.8, 123.2, 121.9, 121.4, 118.7, 107.2, 98.7, 97.4, 86.2, 51.5, 48.7, 29.1, 21.4; HRMS: (ESI) calcd for $\mathrm{C}_{31} \mathrm{H}_{25} \mathrm{NNaO}^{+}[\mathrm{M}+\mathrm{Na}]^{+} 450.1828$; found 450.1844 .

4b,8-Dimethyl-6-phenyl-7-(phenylethynyl)-4b,8-dihydrocyclopenta[4,5]pyrrolo[2,3- $b$ ] pyridin-7a(5H)-ol (3ka)

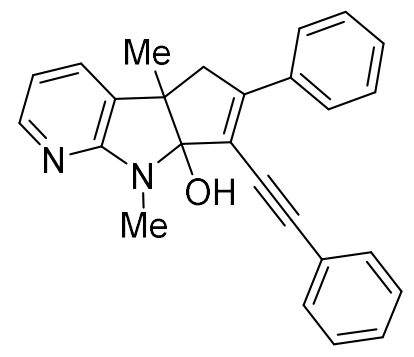

Chemical Formula: $\mathrm{C}_{26} \mathrm{H}_{22} \mathrm{~N}_{2} \mathrm{O}$

Exact Mass: 378.1732

3ka was prepared according to general procedure 2.1 using $\mathbf{1 k}$ and $\mathbf{2 a}$ and was purified by silica gel column chromatography (toluene/EtOAc $=40 / 1 \sim 5 / 1$ ) to obtain $3 \mathbf{k a}$ as yellow solid (60\% yield). mp 61.1-63.5 ${ }^{\circ} \mathrm{C} ;{ }^{1} \mathrm{H}$ NMR (400 MHz, $\left.\mathrm{CDCl}_{3}\right)$ ठ 7.99-7.83 (m, 3H), 7.58$7.48(\mathrm{~m}, 2 \mathrm{H}), 7.47-7.32(\mathrm{~m}, 6 \mathrm{H}), 7.25(\mathrm{dd}, J=7.0,1.6 \mathrm{~Hz}, 1 \mathrm{H}), 6.52(\mathrm{dd}, J=7.0,5.4 \mathrm{~Hz}$, $1 \mathrm{H}), 3.36(\mathrm{~s}, 3 \mathrm{H}), 3.36(\mathrm{bs}, 1 \mathrm{H}), 3.12(\mathrm{~s}, 2 \mathrm{H}), 1.44(\mathrm{~s}, 3 \mathrm{H}) ;{ }^{13} \mathrm{C} \mathrm{NMR}\left(101 \mathrm{MHz}, \mathrm{CDCl}_{3}\right) \delta$ 159.4, 148.1, 146.5, 135.0, 131.5, 129.7, 129.2, 129.0, 128.7, 128.5, 128.3, 127.4, 123.1, $118.8,112.9,105.3,97.6,85.9,50.4,48.9,27.1,20.9$; HRMS: (ESI) calcd for $\mathrm{C}_{26} \mathrm{H}_{23} \mathrm{~N}_{2} \mathrm{O}^{+}[\mathrm{M}+\mathrm{H}]^{+} 379.1805$; found 379.1810 .

4-Benzyl-8b-methyl-2-phenyl-3-(phenylethynyl)-4,8b-dihydrocyclopenta[ $b]$ indol-3a(1H)ol (3ma) 


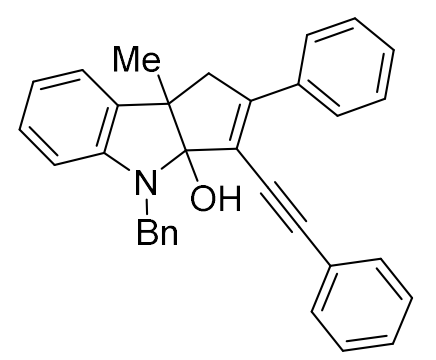

Chemical Formula: $\mathrm{C}_{33} \mathrm{H}_{27} \mathrm{NO}$

Exact Mass: 453.2093

3ma was prepared according to general procedure 2.1 using $1 \mathrm{~m}$ and $2 \mathrm{a}$ and was purified by silica gel column chromatography (toluene/EtOAc $=40 / 1 \sim 5 / 1$ ) to obtain $3 \mathrm{ma}$ as yellow oil (69\% yield). ${ }^{1} \mathrm{H} \mathrm{NMR}\left(400 \mathrm{MHz}, \mathrm{CDCl}_{3}\right)$ ठ 7.99-7.88 (m, 2H), 7.52-7.31 (m, $\left.12 \mathrm{H}\right), 7.30-$ $7.32(\mathrm{~m}, 1 \mathrm{H}), 7.23-7.18(\mathrm{~m}, 1 \mathrm{H}), 7.03(\mathrm{td}, J=7.7,1.3 \mathrm{~Hz}, 1 \mathrm{H}), 6.75(\mathrm{td}, J=7.4,1.0 \mathrm{~Hz}$, $1 \mathrm{H}), 6.25(\mathrm{~d}, J=7.7 \mathrm{~Hz}, 1 \mathrm{H}), 5.08(\mathrm{~d}, J=16.7 \mathrm{~Hz}, 1 \mathrm{H}), 4.94(\mathrm{~d}, J=16.7 \mathrm{~Hz}, 1 \mathrm{H}), 3.31(\mathrm{~d}$, $J=17.1 \mathrm{~Hz}, 1 \mathrm{H}), 3.21(\mathrm{~d}, J=17.2 \mathrm{~Hz}, 1 \mathrm{H}), 2.75(\mathrm{~s}, 1 \mathrm{H}), 1.59(\mathrm{~s}, 3 \mathrm{H}) ;{ }^{13} \mathrm{C} \mathrm{NMR}(101 \mathrm{MHz}$, $\left.\mathrm{CDCl}_{3}\right) \delta 148.4,147.6,139.5,135.3,135.1,131.4,128.9,128.5,128.4,128.3,128.1$, 127.5, 126.8, 126.7, 123.2, 122.5, 119.2, 117.8, 107.4, 106.5, 96.9, 86.1, 52.3, 48.9, 46.9, 21.2; HRMS: (ESI) calcd for $\mathrm{C}_{33} \mathrm{H}_{27} \mathrm{NNaO}^{+}[\mathrm{M}+\mathrm{Na}]^{+} 476.1985$; found 476.1981.

8b-(Methoxymethyl)-4-methyl-2-phenyl-3-(phenylethynyl)-4,8b-dihydrocyclopenta[ $[b]$ indol-3a(1H)-ol (3na)

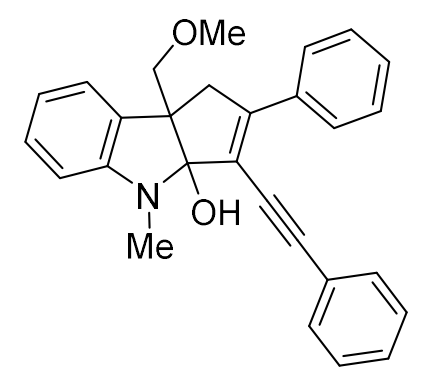

Chemical Formula: $\mathrm{C}_{28} \mathrm{H}_{25} \mathrm{NO}_{2}$

Exact Mass: 407.1885

3na was prepared according to general procedure 2.1 using $\mathbf{1 n}$ and $\mathbf{2 a}$ and was purified by silica gel column chromatography (toluene/EtOAc $=40 / 1 \sim 5 / 1$ ) to obtain 3 na as yellow oil (63\% yield). ${ }^{1} \mathrm{H}$ NMR (400 MHz, $\left.\mathrm{CDCl}_{3}\right) \delta$ 7.95-7.86 (m, 2H), 7.57-7.48 (m, 2H), 7.43$7.29(\mathrm{~m}, 6 \mathrm{H}), 7.16(\mathrm{td}, J=7.7,1.3 \mathrm{~Hz}, 1 \mathrm{H}), 7.10(\mathrm{dd}, J=7.3,1.2 \mathrm{~Hz}, 1 \mathrm{H}), 6.70(\mathrm{td}, J=$ 
7.4, $1.0 \mathrm{~Hz}, 1 \mathrm{H}$ ), 6.42 (d, J = 7.7 Hz, 1H), 4.26 (bs, 1H), 3.88 (d, J = 9.3 Hz, 1H), 3.84 (d, $J=9.3 \mathrm{~Hz}, 1 \mathrm{H}$ ), $3.36(\mathrm{~s}, 3 \mathrm{H}), 3.24(\mathrm{~d}, J=17.3 \mathrm{~Hz}, 1 \mathrm{H}), 3.23(\mathrm{~s}, 3 \mathrm{H}), 3.07$ (d, $J=17.3 \mathrm{~Hz}$, $1 \mathrm{H}) ;{ }^{13} \mathrm{C} N M R\left(101 \mathrm{MHz}, \mathrm{CDCl}_{3}\right) \delta 149.4,146.7,135.2,131.4,131.2,128.8,128.7,128.4$, 128.4, 128.1, 127.5, 123.5, 122.7, 119.8, 117.1, 107.6, 105.2, 96.8, 86.4, 75.8, 59.4, 54.8, 45.0, 28.8; HRMS: (ESI) calcd for $\mathrm{C}_{28} \mathrm{H}_{25} \mathrm{NNaO}_{2}{ }^{+}[\mathrm{M}+\mathrm{Na}]^{+} 430.1778$; found 430.1783 .

8b-Benzyl-4-methyl-2-phenyl-3-(phenylethynyl)-4,8b-dihydrocyclopenta[ $b]$ indol-3a(1H)ol (3oa)

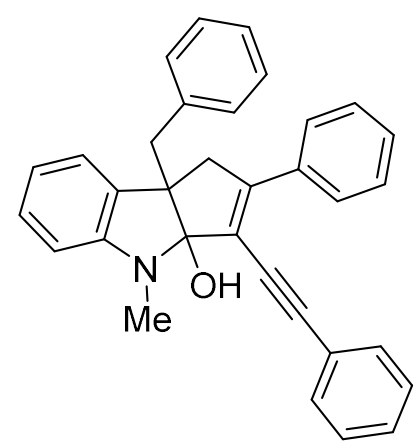

Chemical Formula: $\mathrm{C}_{33} \mathrm{H}_{27} \mathrm{NO}$

Exact Mass: 453.2093

3oa was prepared according to general procedure 2.1 using $\mathbf{1 0}$ and $\mathbf{2 a}$ and was purified by silica gel column chromatography (toluene/EtOAc $=40 / 1 \sim 5 / 1$ ) to obtain 3oa as yellow oil (60\% yield). ${ }^{1} \mathrm{H}$ NMR $\left(400 \mathrm{MHz}, \mathrm{CDCl}_{3}\right) \delta$ 7.91-7.85 (m, 2H), 7.53-7.47 (m, 2H), 7.42$7.35(\mathrm{~m}, 5 \mathrm{H}), 7.33-7.27(\mathrm{~m}, 3 \mathrm{H}), 7.20-7.08(\mathrm{~m}, 4 \mathrm{H}), 6.62-6.55(\mathrm{~m}, 1 \mathrm{H}), 6.50-6.40(\mathrm{~m}, 2 \mathrm{H})$, $3.47(\mathrm{~d}, J=17.0 \mathrm{~Hz}, 1 \mathrm{H}), 3.26(\mathrm{~s}, 3 \mathrm{H}), 3.25(\mathrm{~d}, J=17.0 \mathrm{~Hz}, 1 \mathrm{H}), 3.05(\mathrm{~d}, J=16.9 \mathrm{~Hz}$, 1H), $2.96(\mathrm{~d}, J=17.0 \mathrm{~Hz}, 1 \mathrm{H}), 2.69(\mathrm{bs}, 1 \mathrm{H}) ;{ }^{13} \mathrm{C} \mathrm{NMR}\left(101 \mathrm{MHz}, \mathrm{CDCl}_{3}\right) \delta$ 148.3, 137.8, 135.2, 132.8, 131.3, 130.8, 128.8, 128.5, 128.1, 127.9, 127.6, 126.5, 124.7, 123.4, 118.1, 117.2, 105.7, 96.9, 86.5, 54.5, 45.5, 39.9, 29.5; HRMS: (ESI) calcd for $\mathrm{C}_{33} \mathrm{H}_{27} \mathrm{NNaO}+[\mathrm{M}+\mathrm{Na}]^{+} 476.1985$; found 476.1982 .

4-Methyl-2,8b-diphenyl-3-(phenylethynyl)-4,8b-dihydrocyclopenta[b]indol-3a(1H)-ol (3pa) 


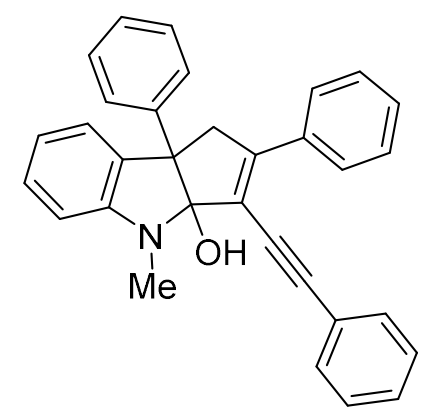

Chemical Formula: $\mathrm{C}_{32} \mathrm{H}_{25} \mathrm{NO}$

Exact Mass: 439.1936

3pa was prepared according to general procedure 2.1 using $1 \mathrm{p}$ and $\mathbf{2 a}$ and was purified by silica gel column chromatography (toluene/EtOAc $=40 / 1 \sim 5 / 1$ ) to obtain 3pa as yellow oil (61\% yield). ${ }^{1} \mathrm{H} \mathrm{NMR}\left(400 \mathrm{MHz}, \mathrm{CDCl}_{3}\right) \delta 8.03(\mathrm{~d}, J=7.4 \mathrm{~Hz}, 2 \mathrm{H}), 7.58-7.52(\mathrm{~m}, 2 \mathrm{H})$, 7.51-7.44 (m, 2H), 7.44-7.37 (m, 6H), 7.36-7.32 (m, 3H), 7.31-7.27 (m, 1H), 7.18-7.13 (m, $1 \mathrm{H}), 6.83$ (t, $J=7.4 \mathrm{~Hz}, 1 \mathrm{H}), 6.58(\mathrm{~d}, J=7.8 \mathrm{~Hz}, 1 \mathrm{H}), 3.96(\mathrm{~d}, J=17.5 \mathrm{~Hz}, 1 \mathrm{H}), 3.53(\mathrm{~d}$, $J=17.5 \mathrm{~Hz}, 1 \mathrm{H}$ ), 3.26 (s, 3H), 1.99 (bs, $1 \mathrm{H}) ;{ }^{13} \mathrm{C} \mathrm{NMR}\left(101 \mathrm{MHz}, \mathrm{CDCl}_{3}\right) \delta$ 149.4, 148.3, 139.7, 135.1, 133.7, 131.4, 129.1, 128.9, 128.7, 128.47, 128.46, 128.29, 128.26, 127.6, 127.4, 124.8, 123.4, 118.3, 118.1, 108.0, 106.1, 96.8, 86.7, 60.5, 48.1, 29.4; HRMS: (ESI) calcd for $\mathrm{C}_{32} \mathrm{H}_{25} \mathrm{NNaO}^{+}[\mathrm{M}+\mathrm{Na}]^{+} 462.1828$; found 462.1832 .

4,8b-Dimethyl-2-(4-pentylphenyl)-3-((4-pentylphenyl)ethynyl)-4,8b-dihydrocyclopenta[ $b]$ indol-3a(1H)-ol (3ab)

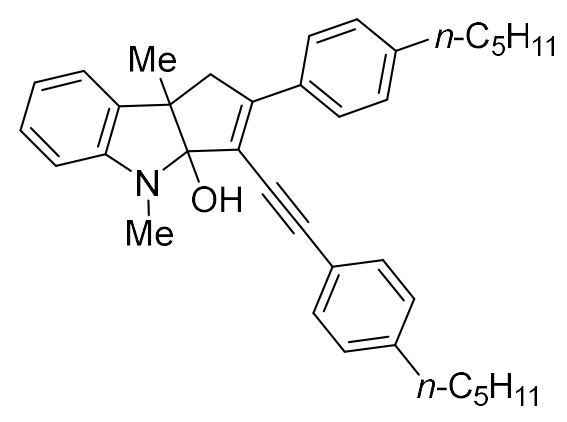

Chemical Formula: $\mathrm{C}_{37} \mathrm{H}_{43} \mathrm{NO}$

Exact Mass: 517.3345

3ab was prepared according to general procedure 2.1 using $\mathbf{1 a}$ and $\mathbf{2} \mathbf{b}$ and was purified by silica gel column chromatography (toluene/EtOAc $=40 / 1 \sim 5 / 1$ ) to obtain 3ab as yellow oil (68\% yield). ${ }^{1} \mathrm{H}$ NMR $\left(400 \mathrm{MHz}, \mathrm{CDCl}_{3}\right) \delta 7.87(\mathrm{~d}, J=8.3 \mathrm{~Hz}, 2 \mathrm{H}), 7.48(\mathrm{~d}, J=8.2 \mathrm{~Hz}$, 
2H), 7.27-7.20 (m, 4H), 7.19-7.13 (m, 2H), 6.77-6.71 (m, 1H), 6.49-6.40 (m, 1H), $3.22(\mathrm{~s}$, $3 \mathrm{H}), 3.20(\mathrm{~d}, J=9.3 \mathrm{~Hz}, 1 \mathrm{H}), 3.11(\mathrm{~d}, J=17.0 \mathrm{~Hz}, 1 \mathrm{H}), 2.73-2.55(\mathrm{~m}, 4 \mathrm{H}), 2.65(\mathrm{bs}, 1 \mathrm{H})$, 1.76-1.56 (m, 4H), $1.49(\mathrm{~s}, 3 \mathrm{H}), 1.44-1.29(\mathrm{~m}, 8 \mathrm{H}), 1.04-0.82(\mathrm{~m}, 6 \mathrm{H}) ;{ }^{13} \mathrm{C} N M R(101 \mathrm{MHz}$, $\left.\mathrm{CDCl}_{3}\right) \delta 148.5,143.9,143.7,135.3,132.7,131.3,128.6,128.2,128.1,127.4,122.5$, $120.6,117.7,117.5,105.5,97.5,86.0,51.8,48.7,36.0,35.8,31.5,31.5,31.0,29.0,22.6$, 21.1, 14.1; HRMS: (ESI) calcd for $\mathrm{C}_{37} \mathrm{H}_{43} \mathrm{NNaO}^{+}[\mathrm{M}+\mathrm{Na}]^{+} 540.3237$; found 540.3205 .

2-([1,1'-Biphenyl]-4-yl)-3-([1,1'-biphenyl]-4-ylethynyl)-4,8b-dimethyl-4,8b-dihydro cyclopenta[b]indol-3a(1H)-ol (3ac)

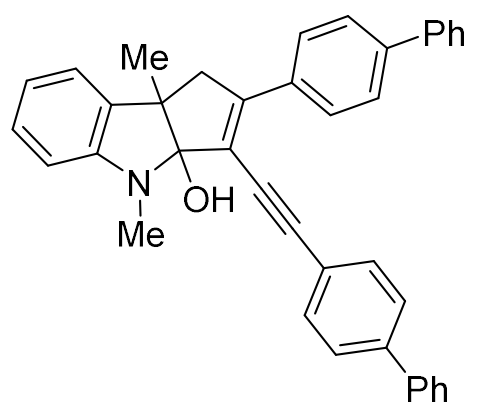

Chemical Formula: $\mathrm{C}_{39} \mathrm{H}_{31} \mathrm{NO}$

Exact Mass: 529.2406

3ac was prepared according to general procedure 2.1 using $\mathbf{1 a}$ and $\mathbf{2 c}$ and was purified by silica gel column chromatography (toluene/EtOAc $=40 / 1 \sim 5 / 1$ ) to obtain 3ac as yellow oil (51\% yield). ${ }^{1} \mathrm{H}$ NMR (400 MHz, $\left.\mathrm{CDCl}_{3}\right)$ ס 8.05-7.96 (m, 2H), 7.70-7.58 (m, 10H), 7.52$7.43(\mathrm{~m}, 5 \mathrm{H}), 7.42-7.34(\mathrm{~m}, 3 \mathrm{H}), 7.20-7.13(\mathrm{~m}, 2 \mathrm{H}), 6.75(\mathrm{t}, J=7.3 \mathrm{~Hz}, 1 \mathrm{H}), 6.46(\mathrm{~d}, J=$ $7.9 \mathrm{~Hz}, 1 \mathrm{H}), 3.24(\mathrm{~s}, 3 \mathrm{H}), 3.25(\mathrm{~d}, J=17.1 \mathrm{~Hz}, 1 \mathrm{H}), 3.15(\mathrm{~d}, J=17.1 \mathrm{~Hz}, 1 \mathrm{H}), 2.59$ (bs, 1H), 1.49 (s, 3H); $\left.{ }^{13} \mathrm{C} \mathrm{NMR} \mathrm{(101} \mathrm{MHz,} \mathrm{CDCl}_{3}\right) \delta 148.4,141.4,141.3,140.5,140.3,135.2$, $134.3,131.9,129.1,129.0,128.9,128.3,127.9,127.8,127.6,127.2,127.08,127.07$, 126.8, 122.5, 122.2, 118.6, 117.7, 105.6, 97.5, 87.3, 51.9, 48.7, 29.1, 21.1; HRMS: (ESI) calcd for $\mathrm{C}_{39} \mathrm{H}_{31} \mathrm{NNaO}^{+}[\mathrm{M}+\mathrm{Na}]^{+} 552.2298$; found 552.2319 .

4,8b-Dimethyl-2-(4-(trifluoromethyl)phenyl)-3-((4-(trifluoromethyl)phenyl)ethynyl)-4,8bdihydrocyclopenta[b]indol-3a(1H)-ol (3ad) 


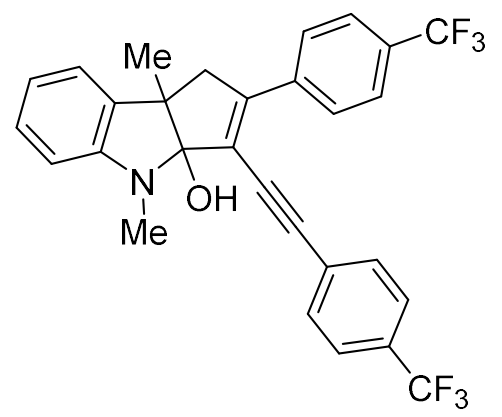

Chemical Formula: $\mathrm{C}_{29} \mathrm{H}_{21} \mathrm{~F}_{6} \mathrm{NO}$

Exact Mass: 513.1527

3ad was prepared according to general procedure 2.1 using $\mathbf{1 a}$ and $\mathbf{2} \mathbf{d}$ and was purified by silica gel column chromatography (toluene/EtOAc $=40 / 1 \sim 5 / 1$ ) to obtain 3ad as yellow oil (36\% yield). ${ }^{1} \mathrm{H}$ NMR $\left(400 \mathrm{MHz}, \mathrm{CDCl}_{3}\right)$ ס 8.00-7.92 (m, 2H), 7.70-7.64 (m, 4H), 7.63$7.59(\mathrm{~m}, 2 \mathrm{H}), 7.21-7.13(\mathrm{~m}, 2 \mathrm{H}), 6.82-6.72(\mathrm{~m}, 1 \mathrm{H}), 6.47(\mathrm{~d}, J=7.7 \mathrm{~Hz}, 1 \mathrm{H}), 3.23(\mathrm{~d}, J=$ $17.2 \mathrm{~Hz}, 1 \mathrm{H}), 3.22(\mathrm{~s}, 3 \mathrm{H}), 3.15(\mathrm{~d}, J=17.2 \mathrm{~Hz}, 1 \mathrm{H}), 2.50(\mathrm{bs}, \mathrm{H}), 1.49(\mathrm{~s}, 3 \mathrm{H}) ;{ }^{13} \mathrm{C} N M R$ $\left(101 \mathrm{MHz}, \mathrm{CDCl}_{3}\right) \delta 148.4,148.0,138.5,134.8,131.6,128.4,127.7,126.6,125.9,125.5$ $(q, J=3.7 \mathrm{~Hz}), 125.1(\mathrm{q}, J=4.0 \mathrm{~Hz}), 122.6,120.2,118.1,105.7,96.1,88.1,51.9,48.7$, 29.0, 21.0; ${ }^{19} \mathrm{~F}$ NMR $\left(376 \mathrm{MHz}, \mathrm{CDCl}_{3}\right.$ ): $\delta-62.7,-62.8$; HRMS: (ESI) calcd for $\mathrm{C}_{29} \mathrm{H}_{22} \mathrm{~F}_{6} \mathrm{NO}^{+}[\mathrm{M}+\mathrm{H}]^{+}$514.1600; found 514.1605.

2-(4-Fluorophenyl)-3-((4-fluorophenyl)ethynyl)-4,8b-dimethyl-4,8b-dihydrocyclopenta[ $b]$ indol-3a(1H)-ol (3ae)

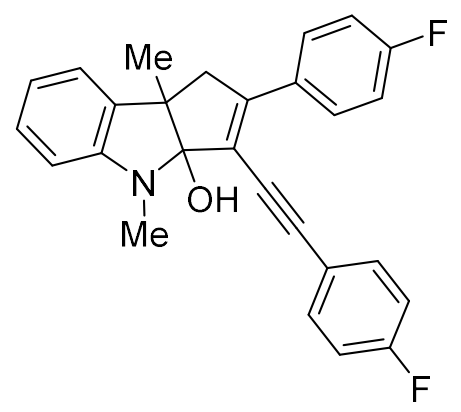

Chemical Formula: $\mathrm{C}_{27} \mathrm{H}_{21} \mathrm{~F}_{2} \mathrm{NO}$

Exact Mass: 413.1591

3ae was prepared according to general procedure 2.1 using $\mathbf{1 a}$ and $\mathbf{2 e}$ and was purified by silica gel column chromatography (toluene/EtOAc $=40 / 1 \sim 5 / 1$ ) to obtain 3ae as yellow oil (64\% yield). ${ }^{1} \mathrm{H}$ NMR (400 MHz, $\left.\mathrm{CDCl}_{3}\right) \delta$ 7.91-7.84 (m, 2H), 7.54-7.46 (m, 2H), 7.19- 
$7.13(\mathrm{~m}, 2 \mathrm{H}), 7.12-7.05(\mathrm{~m}, 4 \mathrm{H}), 6.79-6.71(\mathrm{~m}, 1 \mathrm{H}), 6.45(\mathrm{~d}, J=7.7 \mathrm{~Hz}, 1 \mathrm{H}), 3.21(\mathrm{~s}, 3 \mathrm{H})$, $3.18(\mathrm{~d}, J=17.2 \mathrm{~Hz}, 1 \mathrm{H}), 3.10(\mathrm{~d}, J=17.2 \mathrm{~Hz}, 1 \mathrm{H}), 2.58(\mathrm{bs}, 1 \mathrm{H}), 1.48(\mathrm{~s}, 3 \mathrm{H}) ;{ }^{13} \mathrm{C} \mathrm{NMR}$ $\left(101 \mathrm{MHz}, \mathrm{CDCl}_{3}\right) \delta 163.9(\mathrm{~d}, J=5.2 \mathrm{~Hz}), 161.5(\mathrm{~d}, J=5.8 \mathrm{~Hz}), 148.2,147.5,135.1$, $133.3(\mathrm{~d}, J=8.4 \mathrm{~Hz}$ ), 131.5 (d, $J=3.4 \mathrm{~Hz}), 129.3(\mathrm{~d}, J=8.1 \mathrm{~Hz}), 128.3,122.5,119.3$ (d, $J=3.6 \mathrm{~Hz}), 118.1,117.8,116.0,115.8,115.1(\mathrm{~d}, J=21.5 \mathrm{~Hz}), 105.6,95.9,86.0,51.8$, 48.8, 29.0, 21.1; ${ }^{19} \mathrm{~F} \mathrm{NMR} \mathrm{(376} \mathrm{MHz}, \mathrm{CDCl}_{3}$ ): $\delta$-110.1, -111.6; HRMS: (ESI) calcd for $\mathrm{C}_{27} \mathrm{H}_{21} \mathrm{~F}_{2} \mathrm{NNaO}^{+}[\mathrm{M}+\mathrm{Na}]^{+} 436.1483$; found 436.1489.

2-(4-Chlorophenyl)-3-((4-chlorophenyl)ethynyl)-4,8b-dimethyl-4,8b-dihydrocyclopenta[ $b]$ indol-3a(1H)-ol (3af)

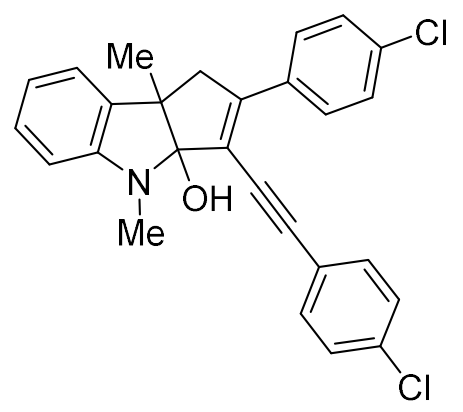

Chemical Formula: $\mathrm{C}_{27} \mathrm{H}_{21} \mathrm{Cl}_{2} \mathrm{NO}$

Exact Mass: 445.1000

3af was prepared according to general procedure 2.1 using $\mathbf{1 a}$ and $\mathbf{2 f}$ and was purified by silica gel column chromatography (toluene/EtOAc $=40 / 1 \sim 5 / 1$ ) to obtain 3af as yellow oil (68\% yield). ${ }^{1} \mathrm{H}$ NMR $\left(400 \mathrm{MHz}, \mathrm{CDCl}_{3}\right) \delta 7.77(\mathrm{~d}, J=8.4 \mathrm{~Hz}, 2 \mathrm{H}), 7.47-7.28(\mathrm{~m}, 6 \mathrm{H})$, 7.20-7.03 (m, 2H), $6.72(\mathrm{t}, J=7.4 \mathrm{~Hz}, 1 \mathrm{H}), 6.42(\mathrm{~d}, J=7.7 \mathrm{~Hz}, 1 \mathrm{H}), 3.17(\mathrm{~s}, 3 \mathrm{H}), 3.15$ (d, $J=17.1 \mathrm{~Hz}, 1 \mathrm{H}), 3.06(\mathrm{~d}, J=17.2 \mathrm{~Hz}, 1 \mathrm{H}), 2.51$ (bs, 1H), $1.44(\mathrm{~s}, 3 \mathrm{H}) ;{ }^{13} \mathrm{C}$ NMR $(101$ $\left.\mathrm{MHz}, \mathrm{CDCl}_{3}\right) \delta 148.1,147.8,135.0,134.7,134.6,133.7,132.6,128.9,128.7,128.4,128.3$, 122.5, 121.6, 118.8, 117.9, 105.6, 96.3, 87.2, 51.8, 48.7, 29.0, 21.1; HRMS: (ESI) calcd for $\mathrm{C}_{27} \mathrm{H}_{22} \mathrm{Cl}_{2} \mathrm{NO}^{+}[\mathrm{M}+\mathrm{H}]^{+} 446.1073$; found 446.1079 .

2-(4-Bromophenyl)-3-((4-bromophenyl)ethynyl)-4,8b-dimethyl-4,8b-dihydrocyclopenta[ $b]$ indol-3a(1H)-ol (3ag) 


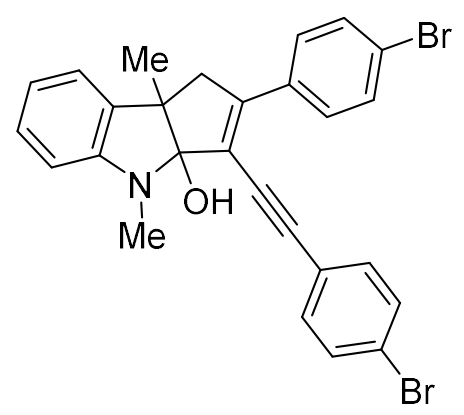

Chemical Formula: $\mathrm{C}_{27} \mathrm{H}_{21} \mathrm{Br}_{2} \mathrm{NO}$

Exact Mass: 532.9990

3ag was prepared according to general procedure 2.1 using $\mathbf{1 a}$ and $\mathbf{2 g}$ and was purified by silica gel column chromatography (toluene/EtOAc $=40 / 1 \sim 5 / 1$ ) to obtain 3ag as yellow oil (63\% yield). ${ }^{1} \mathrm{H}$ NMR (400 MHz, $\left.\mathrm{CDCl}_{3}\right) \delta$ 7.80-7.69 (m, 2H), 7.59-7.49 (m, 4H), 7.40$7.33(\mathrm{~m}, 2 \mathrm{H}), 7.21-7.09(\mathrm{~m}, 2 \mathrm{H}), 6.75(\mathrm{t}, J=7.4 \mathrm{~Hz}, 1 \mathrm{H}), 6.45(\mathrm{~d}, J=7.7 \mathrm{~Hz}, 1 \mathrm{H}), 3.20(\mathrm{~s}$, $3 \mathrm{H}$ ), $3.17(\mathrm{~d}, J=17.2 \mathrm{~Hz}, 1 \mathrm{H}), 3.09$ (d, $J=17.2 \mathrm{~Hz}, 1 \mathrm{H}), 2.56$ (bs, $1 \mathrm{H}), 1.47(\mathrm{~s}, 3 \mathrm{H}) ;{ }^{13} \mathrm{C}$ $\mathrm{NMR}\left(101 \mathrm{MHz}, \mathrm{CDCl}_{3}\right) \delta 148.1,147.9,135.0,134.1,132.7,131.8,131.3,129.0,128.3$, 122.9, 122.9, 122.5, 122.0, 118.9, 117.9, 105.6, 96.4, 87.3, 51.8, 48.6, 29.0, 21.1; HRMS: (ESI) calcd for $\mathrm{C}_{27} \mathrm{H}_{22} \mathrm{Br}_{2} \mathrm{NO}^{+}[\mathrm{M}+\mathrm{H}]^{+} 534.0063$; found 534.0064 .

4,8b-Dimethyl-2-(m-tolyl)-3-(m-tolylethynyl)-4,8b-dihydrocyclopenta[b]indol-3a(1H)-ol (3ah)

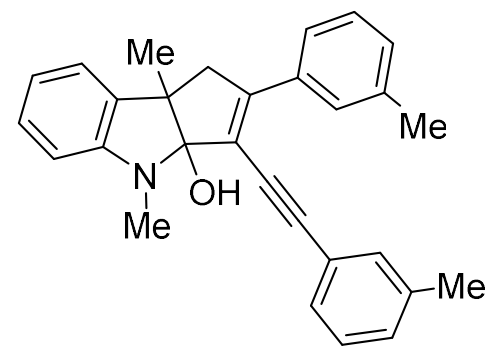

Chemical Formula: $\mathrm{C}_{29} \mathrm{H}_{27} \mathrm{NO}$

Exact Mass: 405.2093

3ah was prepared according to general procedure 2.1 using $\mathbf{1 a}$ and $\mathbf{2 h}$ and was purified by silica gel column chromatography (toluene/EtOAc $=40 / 1 \sim 5 / 1$ ) to obtain 3ah as yellow oil $\left(73 \%\right.$ yield). ${ }^{1} \mathrm{H} \mathrm{NMR}\left(400 \mathrm{MHz}, \mathrm{CDCl}_{3}\right) \delta$ 7.85-7.77 (m, $\left.1 \mathrm{H}\right), 7.72(\mathrm{~d}, J=7.9 \mathrm{~Hz}, 1 \mathrm{H})$, 7.44-7.26 (m, 4H), 7.25-7.12 (m, 4H), 6.76 (td, $J=7.4,1.0 \mathrm{~Hz}, 1 \mathrm{H}), 6.47$ (d, $J=7.7 \mathrm{~Hz}$, 1H), $3.25(\mathrm{~s}, 3 \mathrm{H}), 3.23(\mathrm{~d}, J=9.3 \mathrm{~Hz}, 1 \mathrm{H}), 3.14(\mathrm{~d}, J=9.3 \mathrm{~Hz}, 1 \mathrm{H}), 2.64(\mathrm{bs}, 1 \mathrm{H}), 2.43(\mathrm{~s}$, 
3H), $2.42(\mathrm{~s}, 3 \mathrm{H}), 1.50(\mathrm{~s}, 3 \mathrm{H}) ;{ }^{13} \mathrm{C}$ NMR $\left(101 \mathrm{MHz}, \mathrm{CDCl}_{3}\right) \delta$ 148.4, 138.2, 137.6, 135.3, 131.9, 129.6, 129.4, 128.5, 128.4, 128.2, 128.1, 124.6, 123.3, 122.5, 118.4, 117.6, 105.5, 97.4, 86.3, 51.9, 48.8, 29.1, 21.6, 21.4, 21.1; HRMS: (ESI) calcd for $\mathrm{C}_{29} \mathrm{H}_{27} \mathrm{NNaO}+[\mathrm{M}+\mathrm{Na}]^{+} 428.1985$; found 428.1996 .

2-(3-Methoxyphenyl)-3-((3-methoxyphenyl)ethynyl)-4,8b-dimethyl-4,8b-dihydro cyclopenta[b]indol-3a(1H)-ol (3ai)

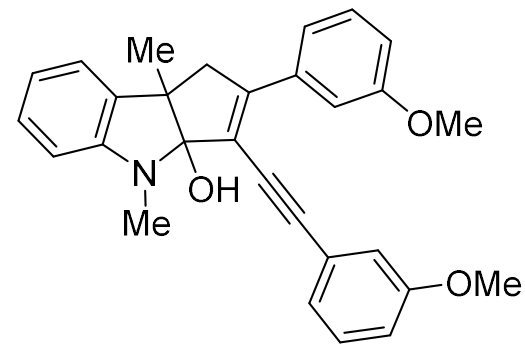

Chemical Formula: $\mathrm{C}_{29} \mathrm{H}_{27} \mathrm{NO}_{3}$

Exact Mass: 437.1991

3ai was prepared according to general procedure 2.1 using $\mathbf{1 a}$ and $\mathbf{2} \mathbf{i}$ and was purified by silica gel column chromatography (toluene/EtOAc $=40 / 1 \sim 5 / 1$ ) to obtain 3ai as yellow oil (58\% yield). ${ }^{1} \mathrm{H}$ NMR $\left(400 \mathrm{MHz}, \mathrm{CDCl}_{3}\right) \delta$ 7.66-7.60 (m, 1H), 7.42-7.37 (m, 1H), 7.35$7.27(\mathrm{~m}, 2 \mathrm{H}), 7.21-7.12(\mathrm{~m}, 3 \mathrm{H}), 7.10-7.05(\mathrm{~m}, 1 \mathrm{H}), 6.99-6.87(\mathrm{~m}, 2 \mathrm{H}), 6.76(\mathrm{td}, J=7.4$, $1.0 \mathrm{~Hz}, 1 \mathrm{H}), 6.46(\mathrm{~d}, J=7.7 \mathrm{~Hz}, 1 \mathrm{H}), 3.86(\mathrm{~s}, 3 \mathrm{H}), 3.82(\mathrm{~s}, 3 \mathrm{H}), 3.23(\mathrm{~s}, 3 \mathrm{H}), 3.21(\mathrm{~d}, J=$ $9.3 \mathrm{~Hz}, 1 \mathrm{H}$ ), $3.12(\mathrm{~d}, J=9.3 \mathrm{~Hz}, 1 \mathrm{H}), 2.67$ (bs, $1 \mathrm{H}), 1.49(\mathrm{~s}, 3 \mathrm{H}) ;{ }^{13} \mathrm{C}$ NMR $(101 \mathrm{MHz}$, $\left.\mathrm{CDCl}_{3}\right) \delta 159.4,159.3,148.6,148.4,136.6,135.2,129.6,129.1,128.2,124.3,123.9$, 122.5, 119.9, 118.7, 117.7, 116.3, 115.0, 114.99, 112.6, 105.5, 97.4, 86.3, 55.3, 55.2, 51.9, 48.8, 29.0, 21.1; HRMS: (ESI) calcd for $\mathrm{C}_{29} \mathrm{H}_{27} \mathrm{NNaO}_{3}{ }^{+}[\mathrm{M}+\mathrm{Na}]^{+} 460.1883$; found 460.1885.

2-(2-Methoxyphenyl)-3-((2-methoxyphenyl)ethynyl)-4,8b-dimethyl-4,8b-dihydro cyclopenta[b]indol-3a(1H)-ol (3aj) 


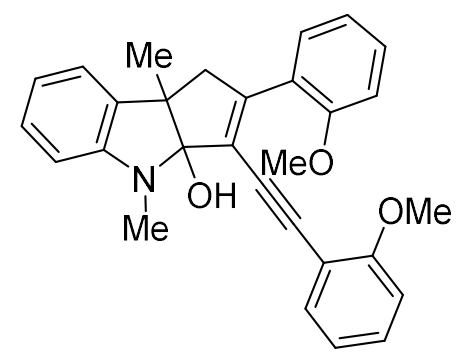

Chemical Formula: $\mathrm{C}_{29} \mathrm{H}_{27} \mathrm{NO}_{3}$

Exact Mass: 437.1991

3aj was prepared according to general procedure 2.1 using $\mathbf{1 a}$ and $\mathbf{2} \mathbf{j}$ and was purified by silica gel column chromatography (toluene/EtOAc $=40 / 1 \sim 5 / 1$ ) to obtain 3aj as yellow oil (45\% yield). ${ }^{1} \mathrm{H}$ NMR (400 MHz, $\left.\mathrm{CDCl}_{3}\right)$ ס 7.82-7.73 (m, 1H), 7.39-7.24 (m, 3H), 7.20$7.09(\mathrm{~m}, 2 \mathrm{H}), 7.02-6.95(\mathrm{~m}, 1 \mathrm{H}), 6.94-6.85(\mathrm{~m}, 3 \mathrm{H}), 6.76-6.68(\mathrm{~m}, 1 \mathrm{H}), 6.45(\mathrm{~d}, J=7.7$ $\mathrm{Hz}, 1 \mathrm{H}), 3.87(\mathrm{~s}, 3 \mathrm{H}), 3.80(\mathrm{~s}, 3 \mathrm{H}), 3.20(\mathrm{~s}, 3 \mathrm{H}), 3.18(\mathrm{~d}, J=17.5 \mathrm{~Hz}, 1 \mathrm{H}), 3.12(\mathrm{~d}, J=$ $17.5 \mathrm{~Hz}, 1 \mathrm{H}$ ), 3.02 (bs, $1 \mathrm{H}), 1.48$ (s, 3H); ${ }^{13} \mathrm{C}$ NMR (101 MHz, $\left.\mathrm{CDCl}_{3}\right) \delta 160.2,157.2$, 149.6, 149.1, 135.5, 132.8, 130.6, 129.6, 127.9, 125.2, 122.3, 120.7, 120.4, 120.0, 117.3, $112.7,110.9,110.5,106.6,105.4,92.3,89.8,55.6,55.3,53.3,50.2,28.9,20.5$; HRMS: (ESI) calcd for $\mathrm{C}_{29} \mathrm{H}_{27} \mathrm{NNaO}_{3}{ }^{+}[\mathrm{M}+\mathrm{Na}]^{+} 460.1883$; found 460.1886 .

2-(2-Chlorophenyl)-3-((2-chlorophenyl)ethynyl)-4,8b-dimethyl-4,8b-dihydrocyclopenta $[b]$ indol-3a $(1 H)-$ ol $(3 \mathbf{a k})$

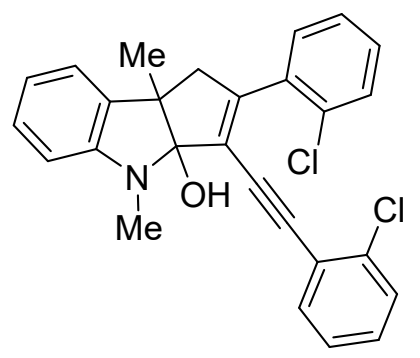

Chemical Formula: $\mathrm{C}_{27} \mathrm{H}_{21} \mathrm{Cl}_{2} \mathrm{NO}$

Exact Mass: 445.1000

3ak was prepared according to general procedure 2.1 using $\mathbf{1 a}$ and $\mathbf{2 k}$ and was purified by silica gel column chromatography (toluene/EtOAc $=40 / 1 \sim 5 / 1$ ) to obtain 3ak as yellow oil (45\% yield). ${ }^{1} \mathrm{H}$ NMR $\left(400 \mathrm{MHz}, \mathrm{CDCl}_{3}\right) \delta$ 7.49-7.39 (m, 2H), 7.38-7.35 (m, 1H), 7.34$7.32(\mathrm{~m}, 1 \mathrm{H}), 7.29-7.11(\mathrm{~m}, 6 \mathrm{H}), 6.79-6.71(\mathrm{~m}, 1 \mathrm{H}), 6.48(\mathrm{~d}, J=7.8 \mathrm{~Hz}, 1 \mathrm{H}), 3.22(\mathrm{~s}, 3 \mathrm{H})$, 
3.15 (s, 2H), 2.61 (bs, 1H), 1.51 (s, 3H); ${ }^{13} \mathrm{C}$ NMR (101 MHz, $\left.\mathrm{CDCl}_{3}\right) \delta$ 152.0, 148.6, 135.8, 135.3, 135.0, 133.2, 132.4, 130.6, 129.8, 129.4, 129.2, 128.2, 126.4, 126.3, 123.0, 122.8, 122.5, 117.8, 106.8, 105.7, 92.6, 89.5, 53.4, 50.1, 29.2, 20.6; HRMS: (ESI) calcd for $\mathrm{C}_{27} \mathrm{H}_{21} \mathrm{Cl}_{2} \mathrm{NNaO}+[\mathrm{M}+\mathrm{Na}]^{+} 468.0892$; found 468.0884 .

4,8b-Dimethyl-2-(naphthalen-2-yl)-3-(naphthalen-2-ylethynyl)-4,8b-dihydrocyclopenta $[b]$ indol-3a $(1 H)$-ol (3al)

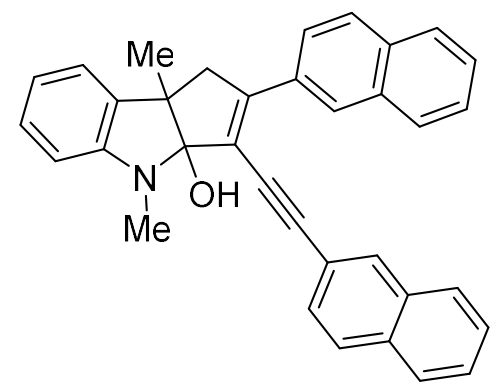

Chemical Formula: $\mathrm{C}_{35} \mathrm{H}_{27} \mathrm{NO}$

Exact Mass: 477.2093

3al was prepared according to general procedure 2.1 using $\mathbf{1 a}$ and $\mathbf{2} \mathbf{I}$ and was purified by silica gel column chromatography (toluene/EtOAc $=40 / 1 \sim 5 / 1$ ) to obtain 3al as yellow oil $\left(73 \%\right.$ yield). ${ }^{1} \mathrm{H}$ NMR $\left(400 \mathrm{MHz}, \mathrm{CDCl}_{3}\right) \delta 8.30(\mathrm{~d}, J=1.7 \mathrm{~Hz}, 1 \mathrm{H}), 8.25-8.18(\mathrm{~m}, 1 \mathrm{H})$, $8.06(\mathrm{~d}, J=1.5 \mathrm{~Hz}, 1 \mathrm{H}), 7.93-7.77(\mathrm{~m}, 6 \mathrm{H}), 7.64-7.58(\mathrm{~m}, 1 \mathrm{H}), 7.57-7.48(\mathrm{~m}, 4 \mathrm{H}), 7.24-$ $7.14(\mathrm{~m}, 2 \mathrm{H}), 6.77(\mathrm{td}, J=7.5,0.9 \mathrm{~Hz}, 1 \mathrm{H}), 6.48(\mathrm{~d}, J=7.6 \mathrm{~Hz}, 1 \mathrm{H}), 3.34(\mathrm{~d}, J=17.0 \mathrm{~Hz}$, 1H), 3.31 (s, 3H), 3.26 (d, J = $17.5 \mathrm{~Hz}, 1 \mathrm{H}), 2.67$ (bs, 1H), 1.53 (s, 3H); ${ }^{13} \mathrm{C}$ NMR (101 $\left.\mathrm{MHz}, \mathrm{CDCl}_{3}\right) \delta 148.7,148.4,135.3,133.4,133.1,132.9,131.3,129.1,128.6,128.3,128.3$, 128.0, 127.9, 127.7, 127.6, 127.3, 126.9, 126.8, 126.7, 126.4, 125.4, 125.1, 122.6, 120.7, $119.1,117.8,105.6,97.9,87.1,52.0,48.9,29.2,21.2$; HRMS: (ESI) calcd for $\mathrm{C}_{35} \mathrm{H}_{28} \mathrm{NO}^{+}[\mathrm{M}+\mathrm{H}]^{+} 478.2165$; found 478.2164 .

4,8b-Dimethyl-2-(thiophen-3-yl)-3-(thiophen-3-ylethynyl)-4,8b-dihydrocyclopenta[ $b]$ indol3a(1H)-ol (3am) 


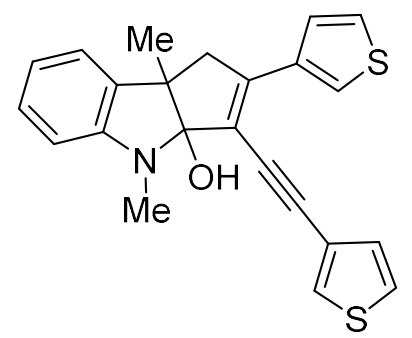

Chemical Formula: $\mathrm{C}_{23} \mathrm{H}_{19} \mathrm{NOS}_{2}$

Exact Mass: 389.0908

3am was prepared according to general procedure 2.1 using $\mathbf{1 a}$ and $\mathbf{2 m}$ and was purified by silica gel column chromatography (toluene/EtOAc $=40 / 1 \sim 5 / 1$ ) to obtain 3am as yellow oil (63\% yield). ${ }^{1} \mathrm{H} \mathrm{NMR}\left(400 \mathrm{MHz}, \mathrm{CDCl}_{3}\right) \delta 7.82(\mathrm{dd}, J=5.1,1.3 \mathrm{~Hz}, 1 \mathrm{H}), 7.75-7.71(\mathrm{~m}$, 1H), 7.60-7.54 (m, 1H), 7.40-7.35 (m, 1H), 7.34-7.32 (m, 1H), 7.27-7.23 (m, 1H), 7.19$7.12(\mathrm{~m}, 2 \mathrm{H}), 6.75(\mathrm{td}, J=7.4,1.0 \mathrm{~Hz}, 1 \mathrm{H}), 6.44(\mathrm{dd}, J=8.2,1.0 \mathrm{~Hz}, 1 \mathrm{H}), 3.20(\mathrm{~s}, 3 \mathrm{H})$, 3.19 (d, $J=17.0 \mathrm{~Hz}, 1 \mathrm{H}$ ), 3.08 (d, $J=17.0 \mathrm{~Hz}, 1 \mathrm{H}), 2.55$ (bs, $1 \mathrm{H}), 1.47(\mathrm{~s}, 3 \mathrm{H}) ;{ }^{13} \mathrm{C} \mathrm{NMR}$ $\left(101 \mathrm{MHz}, \mathrm{CDCl}_{3}\right) \delta 148.3,137.2,135.2,129.6,128.7,128.2,126.6,125.7,125.3,124.9$, 122.5, 122.3, 117.7, 116.9, 105.6, 93.2, 86.1, 51.9, 48.9, 29.1, 21.2; HRMS: (ESI) calcd for $\mathrm{C}_{23} \mathrm{H}_{19} \mathrm{NNaOS}_{2}{ }^{+}[\mathrm{M}+\mathrm{Na}]^{+} 412.0800$; found 412.0803 .

4,8b-Dimethyl-2-(thiophen-2-yl)-3-(thiophen-2-ylethynyl)-4,8b-dihydrocyclopenta[b]indol3a(1H)-ol (3an)

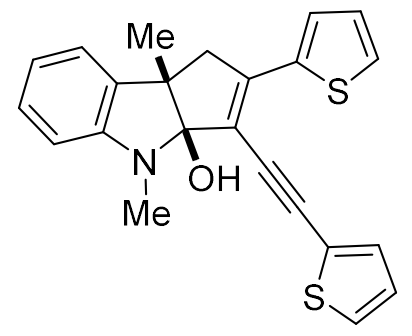

Chemical Formula: $\mathrm{C}_{23} \mathrm{H}_{19} \mathrm{NOS}_{2}$

Exact Mass: 389.0908

3an was prepared according to general procedure 2.1 using $\mathbf{1 a}$ and $\mathbf{2 n}$ and was purified by silica gel column chromatography (toluene/EtOAc $=40 / 1 \sim 5 / 1$ ) to obtain 3an as yellow oil (36\% yield). ${ }^{1} \mathrm{H}$ NMR (400 MHz, $\left.\mathrm{CDCl}_{3}\right) \delta$ 7.41-7.32 (m, 3H), 7.32-7.29 (m, 1H), 7.15$7.09(\mathrm{~m}, 2 \mathrm{H}), 7.07-7.01(\mathrm{~m}, 2 \mathrm{H}), 6.74-6.68(\mathrm{~m}, 1 \mathrm{H}), 6.40(\mathrm{~d}, J=8.0 \mathrm{~Hz}, 1 \mathrm{H}), 3.22(\mathrm{~d}, J=$ $16.8 \mathrm{~Hz}, 1 \mathrm{H}), 3.15(\mathrm{~s}, 3 \mathrm{H}), 3.10(\mathrm{~d}, J=16.9 \mathrm{~Hz}, 1 \mathrm{H}), 2.42(\mathrm{bs}, 1 \mathrm{H}), 1.44(\mathrm{~s}, 3 \mathrm{H}) ;{ }^{13} \mathrm{C} \mathrm{NMR}$ 
$\left(101 \mathrm{MHz}, \mathrm{CDCl}_{3}\right) \delta 148.1,143.2,138.6,135.1,131.9,128.3,128.0,127.8,127.8,127.3$, 126.7, 123.4, 122.5, 117.8, 116.0, 107.3, 105.69, 105.65, 94.1, 52.1, 48.6, 29.8, 21.2; HRMS: (ESI) calcd for $\mathrm{C}_{23} \mathrm{H}_{20} \mathrm{NOS}_{2}+[\mathrm{M}+\mathrm{H}]^{+} 390.0981$; found 390.0980 .

2-(Benzo[b]thiophen-3-yl)-3-(benzo[b]thiophen-3-ylethynyl)-4,8b-dimethyl-4,8b-dihydro cyclopenta[b]indol-3a(1H)-ol (3ao)

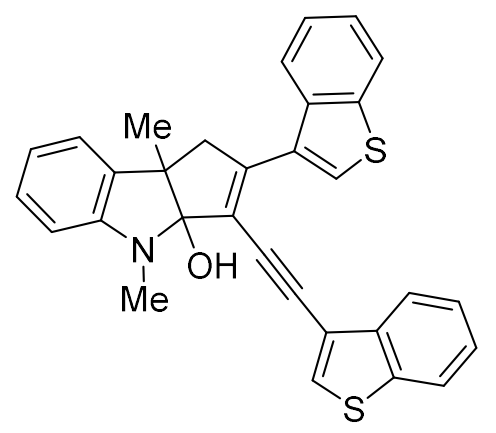

Chemical Formula: $\mathrm{C}_{31} \mathrm{H}_{23} \mathrm{NOS}_{2}$

Exact Mass: 489.1221

3ao was prepared according to general procedure 2.1 using $\mathbf{1 a}$ and $\mathbf{2 0}$ and was purified by silica gel column chromatography (toluene/EtOAc $=40 / 1 \sim 5 / 1$ ) to obtain 3ao as yellow oil (52\% yield). ${ }^{1} \mathrm{H}$ NMR (400 MHz, $\left.\mathrm{CDCl}_{3}\right) \delta$ 8.01-7.90 (m, 2H), 7.86-7.79 (m, 2H), 7.55$7.44(\mathrm{~m}, 2 \mathrm{H}), 7.43-7.34(\mathrm{~m}, 3 \mathrm{H}), 7.33-7.29(\mathrm{~m}, 1 \mathrm{H}), 7.24-7.15(\mathrm{~m}, 2 \mathrm{H}), 6.79(\mathrm{td}, J=7.4$, $0.9 \mathrm{~Hz}, 1 \mathrm{H}), 6.51(\mathrm{~d}, J=7.7 \mathrm{~Hz}, 1 \mathrm{H}), 3.34(\mathrm{~d}, J=17.3 \mathrm{~Hz}, 1 \mathrm{H}), 3.29(\mathrm{~s}, 3 \mathrm{H}), 3.23(\mathrm{~d}, J=$ $17.3 \mathrm{~Hz}, 1 \mathrm{H}), 2.59$ (bs, 1H), 1.55 (s, 3H); ${ }^{13} \mathrm{C}$ NMR (101 MHz, $\left.\mathrm{CDCl}_{3}\right) \delta 148.4,140.2$, 138.9, 138.6, 137.1, 132.5, 129.8, 128.3, 126.3, 125.0, 124.7, 124.5, 124.3, 124.1, 123.0, 122.8, 122.6, 122.5, 120.5, 118.3, 117.9, 105.8, 91.1, 88.1, 52.9, 50.7, 29.3, 20.9; HRMS: (ESI) calcd for $\mathrm{C}_{31} \mathrm{H}_{24} \mathrm{NOS}_{2}+[\mathrm{M}+\mathrm{H}]^{+} 490.1294$; found 490.1270 . 
(3aR,8bS)-2,4,8b-trimethyl-3-phenyl-1,3a,4,8b-tetrahydrocyclopenta[b]indole (8aa)

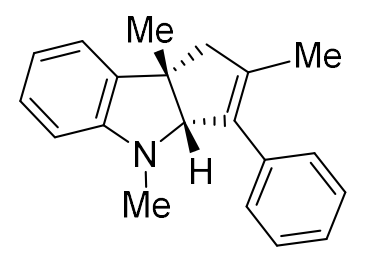

Chemical Formula: $\mathrm{C}_{20} \mathrm{H}_{21} \mathrm{~N}$

Exact Mass: 275.1674

8aa was prepared according to general procedure 2.3 using $1 \mathbf{a}$ and $7 \mathbf{a}$ and was purified by silica gel column chromatography (petroleum ether/toluene 2:1-1:99) to obtain 8aa as white solid (60\% yield). mp 41.6-43.2 ${ }^{\circ} \mathrm{C} ;{ }^{1} \mathrm{H} \mathrm{NMR}\left(400 \mathrm{MHz}, \mathrm{CDCl}_{3}\right) \delta$ 7.52-7.23 (m, 5H), 7.18-7.04 (m, 2H), $6.70(\mathrm{t}, J=7.3 \mathrm{~Hz}, 1 \mathrm{H}), 6.36(\mathrm{~d}, J=8.0 \mathrm{~Hz}, 1 \mathrm{H}), 4.55(\mathrm{~s}, 1 \mathrm{H}), 2.92(\mathrm{~d}$, $J=16.7 \mathrm{~Hz}, 1 \mathrm{H}), 2.63(\mathrm{~d}, J=16.7 \mathrm{~Hz}, 1 \mathrm{H}), 2.54(\mathrm{~s}, 3 \mathrm{H}), 1.75(\mathrm{~s}, 3 \mathrm{H}), 1.56(\mathrm{~s}, 3 \mathrm{H}) ;{ }^{13} \mathrm{C}$ NMR $\left(101 \mathrm{MHz}, \mathrm{CDCl}_{3}\right) \delta 151.2,138.1,138.0,137.7,135.9,128.5,128.2,127.8,126.5$, 122.5, 116.8, 106.3, 86.9, 54.0, 49.5, 35.2, 28.0, 15.1; HRMS: (ESI) calcd for $\mathrm{C}_{20} \mathrm{H}_{22} \mathrm{~N}^{+}[\mathrm{M}+\mathrm{H}]^{+} 276.1747$; found 276.1747 .

The enantiomeric purity was established by HPLC analysis using a chiral column: OJ-H column, $30{ }^{\circ} \mathrm{C}, n$-Hexane $/ i$-Propanol $=95 / 5$ as eluent, $254 \mathrm{~nm}, 1 \mathrm{~mL} / \mathrm{min}$. tR $=4 \mathrm{~min}$ (major), 9 min (minor).

Optical Rotation: $[\alpha]_{D}{ }^{23}-88.7$ (c 0.4 , $\mathrm{PrOH}$ ) for $90 \%$ ee. 


\section{LabSolutions J柜护 $\frac{H}{\square} \leftarrow$ analysis report}

〈样品信息〉

样品名
样品ID
数据文件名
方法文件名
批处理文件名
样品瓶号
进样体积
分析旦期
处理日期

: pyy-3-247 $\quad$ Sample name

: pyy-3-247. 1cd $\longleftarrow$ Data file name

OJ-H-5\%iPropanol-1mL-15min. Icm $\longleftrightarrow$ Method file name

: $1.1 \mathrm{cb}$

: 1-5 样品类型 : 末知

: $8 \mathrm{uL} \leftarrow$ Injection volume

: 2019/1/7 17:40:51下 分析者过 System Administrator

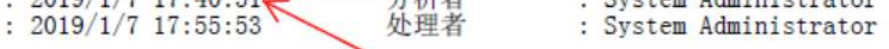

\begin{tabular}{ll}
\hline 〈色谱图〉 Analysis date \\
maU
\end{tabular}

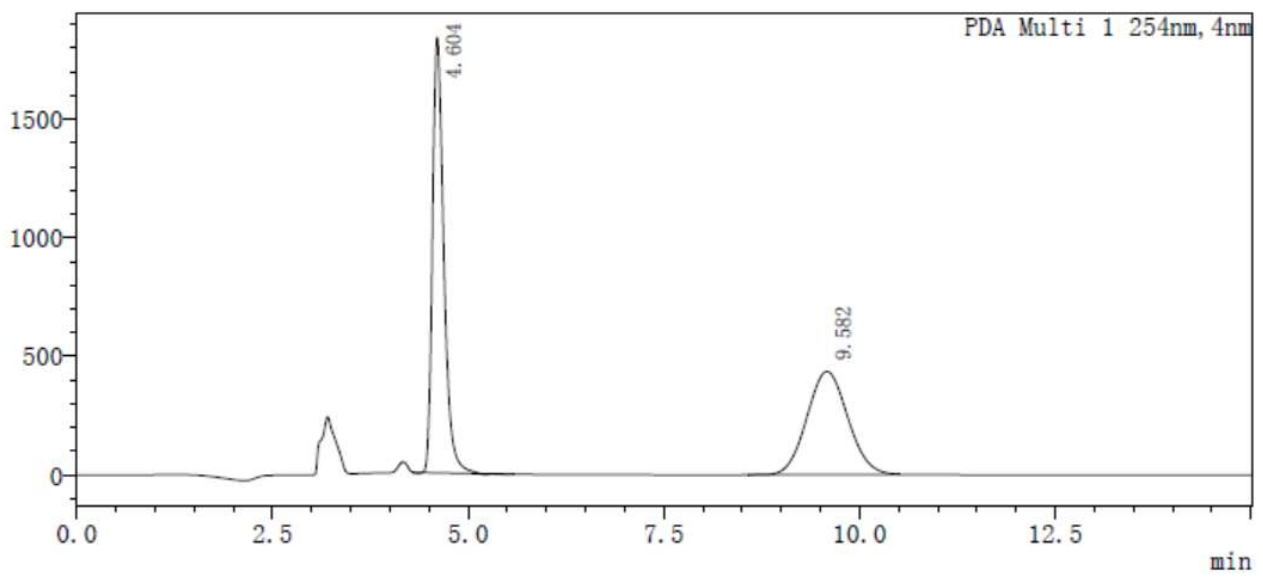

〈峰表〉

PDA Ch1 254nm

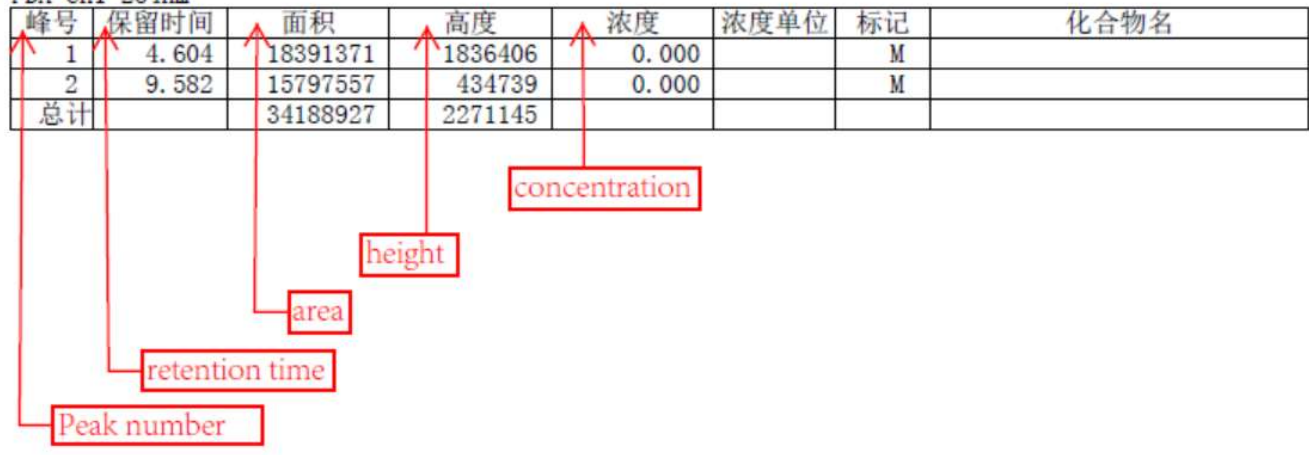




\section{LabSolutlons 分析报告}
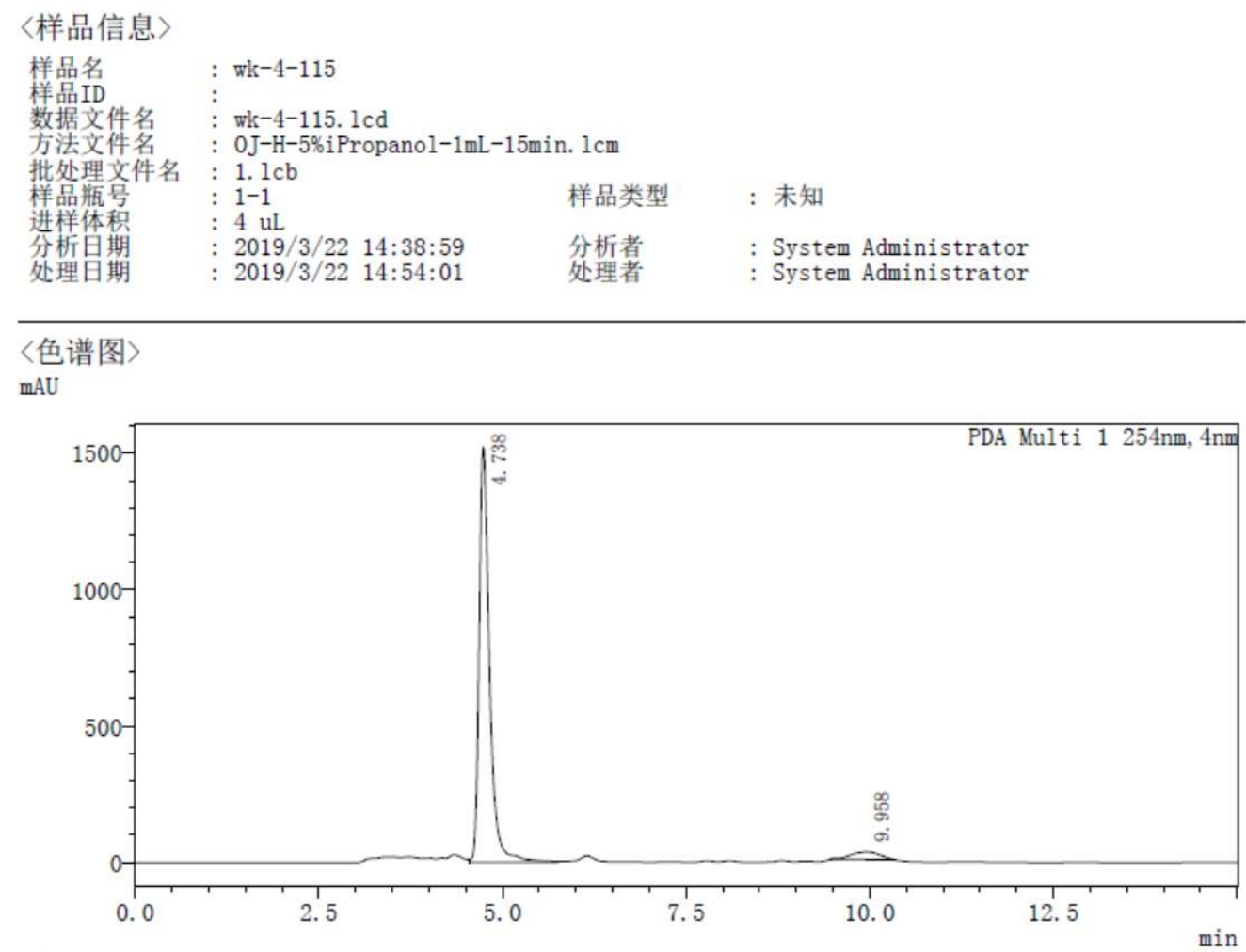

〈峰表〉

PDA Ch1 254nm

\begin{tabular}{|c|c|c|c|c|c|c|c|}
\hline 峰号 & 保留时间 & 丹面积 & 高度 & 浓度 & 浓度单位 & 标记 & 化合物名 \\
\hline 木 1 & $\$ 4.738$ & 14993183 & 1523798 & 0.000 & & M & \\
\hline 2 & 9.958 & 708757 & 26482 & 0.000 & & M & \\
\hline 总计 & & 15701941 & 1550279 & & & & \\
\hline
\end{tabular}

peak number

retention time 
(3aR,8bS)-7-fluoro-2,4,8b-trimethyl-3-phenyl-1,3a,4,8b-tetrahydrocyclopenta[b]indole (8ba)

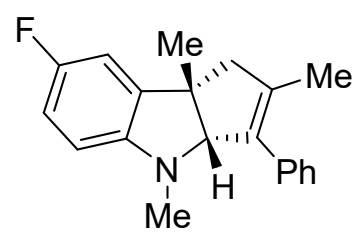

Chemical Formula: $\mathrm{C}_{20} \mathrm{H}_{20} \mathrm{FN}$

Exact Mass: 293.1580

$\mathbf{8 b a}$ was prepared according to general procedure 2.3 using $\mathbf{1 b}$ and $7 \mathrm{a}$ and was purified by silica gel column chromatography (petroleum ether/toluene $2: 1-1: 99$ ) to obtain $8 \mathrm{ba}$ as colorless oil (61\% yield). ${ }^{1} \mathrm{H}$ NMR $\left(400 \mathrm{MHz}, \mathrm{CDCl}_{3}\right) \delta$ 7.37-7.24 (m, 5H), 6.79-6.70 (m, 2H), 6.19 (dd, J = 8.4, $4.1 \mathrm{~Hz}, 1 \mathrm{H}), 4.45(\mathrm{~s}, 1 \mathrm{H}), 2.84(\mathrm{~d}, J=16.5 \mathrm{~Hz}, 1 \mathrm{H}), 2.55(\mathrm{~d}, J=$ $16.7 \mathrm{~Hz}, 1 \mathrm{H}), 2.46(\mathrm{~s}, 3 \mathrm{H}), 1.70(\mathrm{~s}, 3 \mathrm{H}), 1.48(\mathrm{~s}, 3 \mathrm{H}) ;{ }^{13} \mathrm{C} \mathrm{NMR}\left(101 \mathrm{MHz}, \mathrm{CDCl}_{3}\right) \delta 157.5$, 155.2, 147.6, 139.1 (d, $J=7.2 \mathrm{~Hz}$ ), 137.9, 137.7, 135.9, 128.3 (d, $J=25.5 \mathrm{~Hz}$ ), 126.6, $113.4(\mathrm{~d}, J=22.8 \mathrm{~Hz}), 110.1$ (d, $J=23.8 \mathrm{~Hz}), 106.6(\mathrm{~d}, J=8.1 \mathrm{~Hz}), 87.5,53.6,49.6,36.2$, 27.7, 15.0; HRMS: (ESI) calcd for $\mathrm{C}_{20} \mathrm{H}_{21} \mathrm{FN}^{+}[\mathrm{M}+\mathrm{H}]^{+}$294.1653; found 294.1642.

The enantiomeric purity was established by HPLC analysis using a chiral column: OJ-H column, $30{ }^{\circ} \mathrm{C}, n$-Hexane/i-Propanol $=95 / 5$ as eluent, $254 \mathrm{~nm}, 1 \mathrm{~mL} / \mathrm{min}$. $\mathrm{tR}=4 \mathrm{~min}$ (major), 7 min (minor).

Optical Rotation: $[\alpha]_{D^{27}}-14.6$ (c 0.4 , $\left.{ }^{i} \mathrm{PrOH}\right)$ for $90 \%$ ee. 


\section{LabSolutions 分析报告}

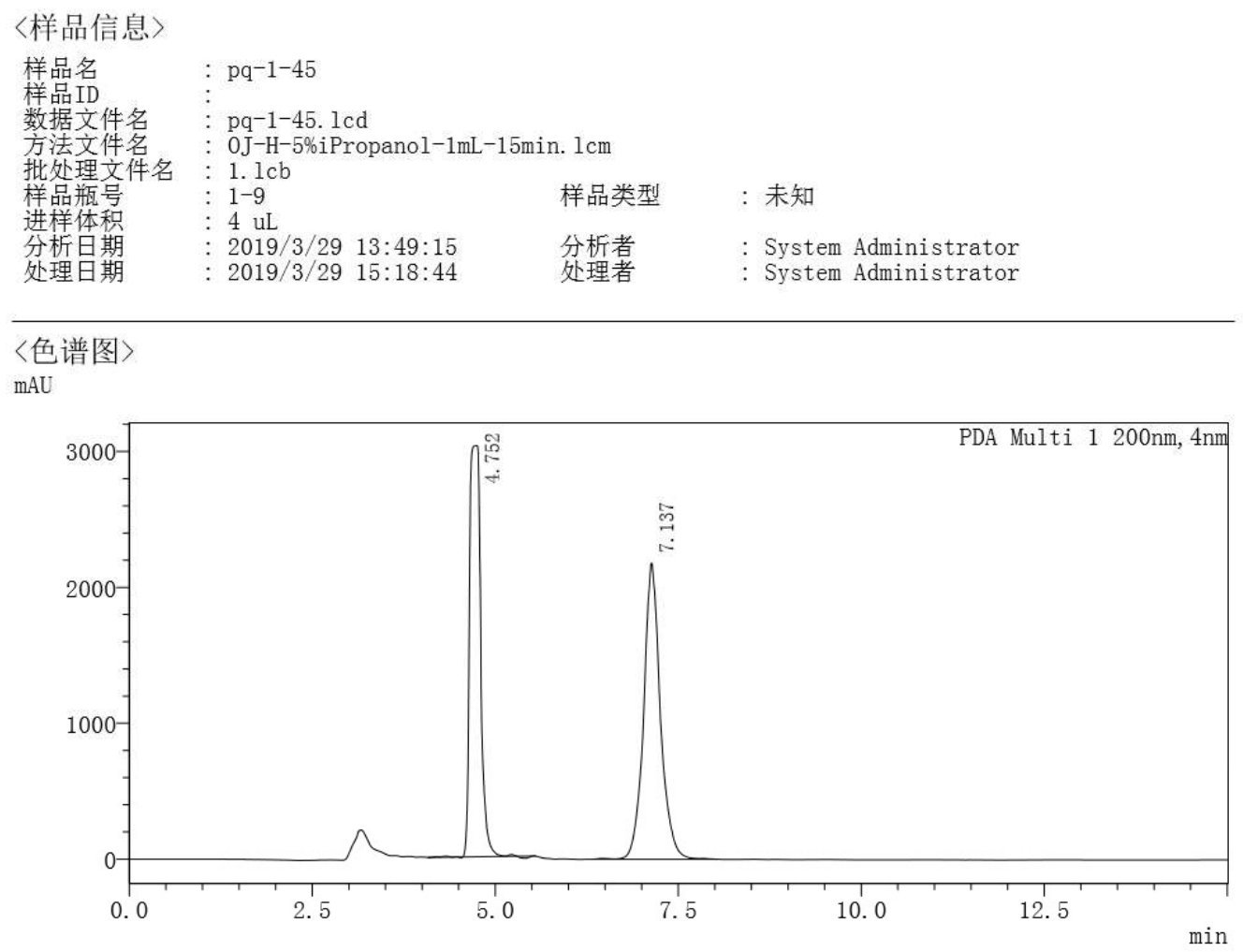

〈峰表〉

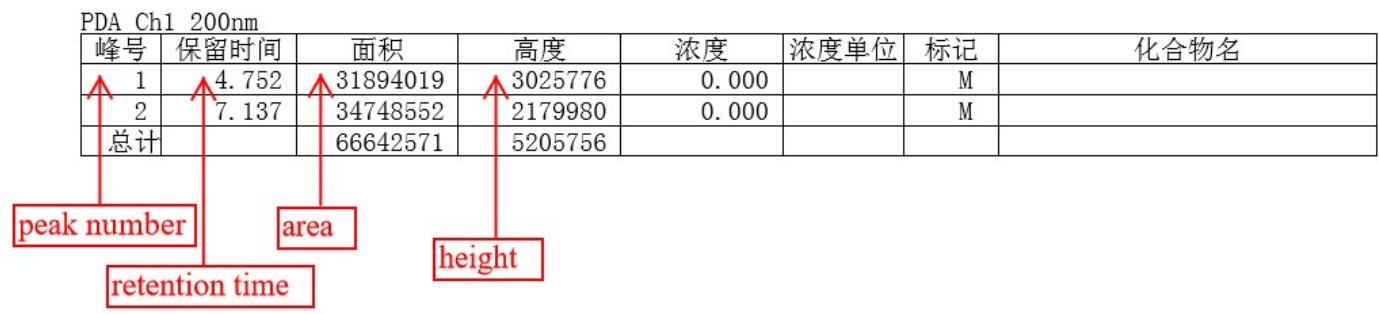




\section{LabSolutions 分析报告}

〈样品信息〉

$\begin{array}{llll}\text { 样品名 } & \vdots \text { wk-4-134 } & \\ \text { 样品ID } & \vdots & \\ \text { 数据文件名 } & \vdots \text { wk-4-134. lcd } & \\ \text { 方法文件名 } & \vdots \text { JJ-H-5\% iPropanol-1mL-15min. } 1 \mathrm{~cm} & \\ \text { 批处理文件名 } & \vdots 1.1 \mathrm{cb} & & \\ \text { 样品瓶号 } & \vdots 1-1 & \text { 样品类型 } & : \text { 未知 } \\ \text { 进样体积 } & \vdots 4 \mathrm{uL} & \\ \text { 分析日期 } & \vdots 2019 / 4 / 220: 59: 05 & \text { 分析者 } & \text { : System Administrator } \\ \text { 处理日期 } & : 2019 / 4 / 221: 13: 42 & \text { 处理者 } & \text { : System Administrator }\end{array}$

〈色谱图〉

$\mathrm{mAU}$

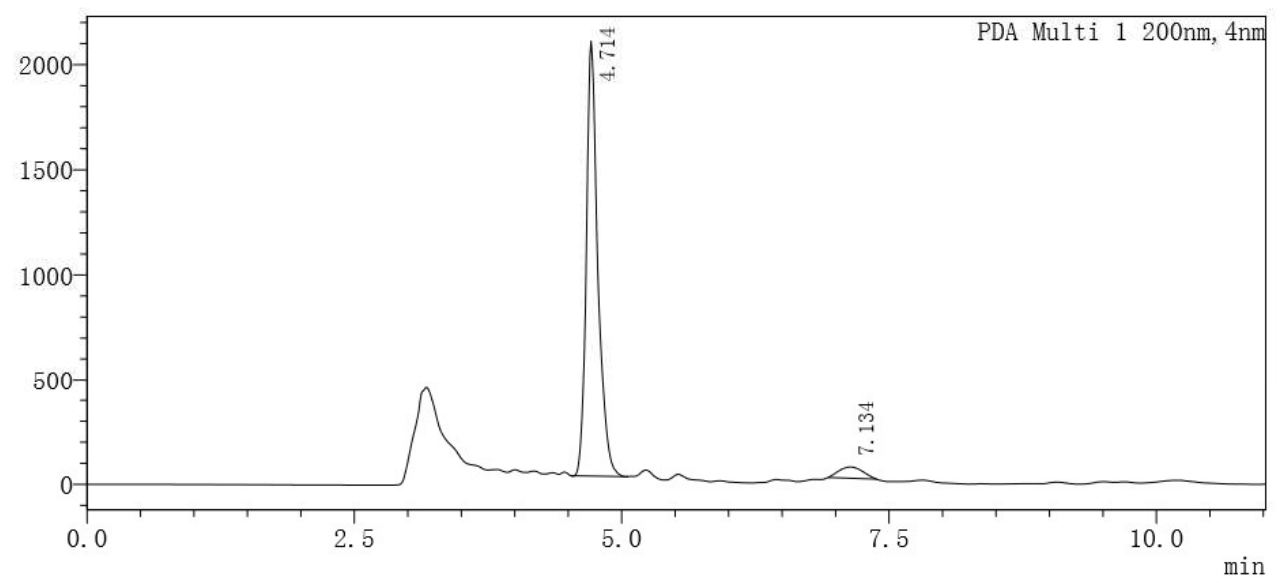

〈峰表〉

\begin{tabular}{|c|c|c|c|c|c|c|c|}
\hline 峰号 & 保留时间 & 面积 & 高度 & 浓度 & 浓度单位 & 标记 & 化合物名 \\
\hline 1 & 4.714 & 15489560 & 2073244 & 0.000 & & M & \\
\hline$\$ 2$ & 7.134 & 1 827984 & 53518 & 0.000 & & M & \\
\hline 总计 & & 16317544 & 2126761 & & & & \\
\hline
\end{tabular}

retention time 
(3aR,8bS)-2,4,8b-trimethyl-3-phenyl-7-(trifluoromethyl)-1,3a,4,8b-

tetrahydrocyclopenta[b]indole (8ca)

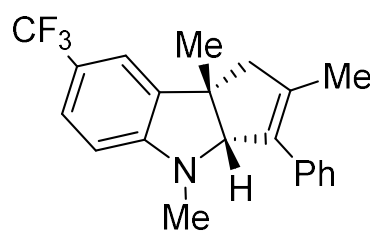

Chemical Formula: $\mathrm{C}_{21} \mathrm{H}_{20} \mathrm{~F}_{3} \mathrm{~N}$

Exact Mass: 343.1548

8ca was prepared according to general procedure 2.3 using $1 \mathrm{c}$ and $7 \mathrm{a}$ and was purified by silica gel column chromatography (petroleum ether/toluene 2:1-1:99) to obtain 8ca as colorless oil (44\% yield). ${ }^{1} \mathrm{H}$ NMR $\left(400 \mathrm{MHz}, \mathrm{CDCl}_{3}\right) \delta$ 7.34-7.25 (m, 2H), 7.24-7.12 (m, $6 \mathrm{H}), 6.14(\mathrm{~d}, J=8.3 \mathrm{~Hz}, 1 \mathrm{H}), 4.56(\mathrm{~s}, 1 \mathrm{H}), 2.80(\mathrm{dt}, J=16.9,1.6 \mathrm{~Hz}, 1 \mathrm{H}), 2.57(\mathrm{~d}, J=$ $16.8 \mathrm{~Hz}, 1 \mathrm{H}), 2.42(\mathrm{~s}, 3 \mathrm{H}), 1.62(\mathrm{~s}, 3 \mathrm{H}), 1.43(\mathrm{~s}, 3 \mathrm{H}) ;{ }^{13} \mathrm{C} \mathrm{NMR}\left(101 \mathrm{MHz}, \mathrm{CDCl}_{3}\right) \delta 152.9$, 138.2, 137.9, 137.5, 135.5, 128.4, 128.3, 126.7, 125.89, 125.85, 119.5 (q, J = $3.3 \mathrm{~Hz}$ ), 104.2, 86.3, 53.8, 49.1, 33.8, 27.8, 14.9; $\left.{ }^{19} \mathrm{~F} \mathrm{NMR} \mathrm{(377} \mathrm{MHz,} \mathrm{CDCl}_{3}\right) \delta-60.13$; HRMS: (ESI) calcd for $\mathrm{C}_{21} \mathrm{H}_{21} \mathrm{~F}_{3} \mathrm{~N}^{+}[\mathrm{M}+\mathrm{H}]^{+} 344.1621$; found 344.1617 .

The enantiomeric purity was established by HPLC analysis using a chiral column: OJ-H column, $30{ }^{\circ} \mathrm{C}, n$-Hexane/i-Propanol $=98 / 2$ as eluent, $254 \mathrm{~nm}, 0.5 \mathrm{~mL} / \mathrm{min}$. $\mathrm{tR}=9 \mathrm{~min}$ (major), 11 min (minor).

Optical Rotation: $[\alpha]_{D}^{23}-59.7$ (c 0.1, $\mathrm{PrOH}$ ) for $92 \%$ ee. 


\section{iㅐ LabSolutions 分析报告}

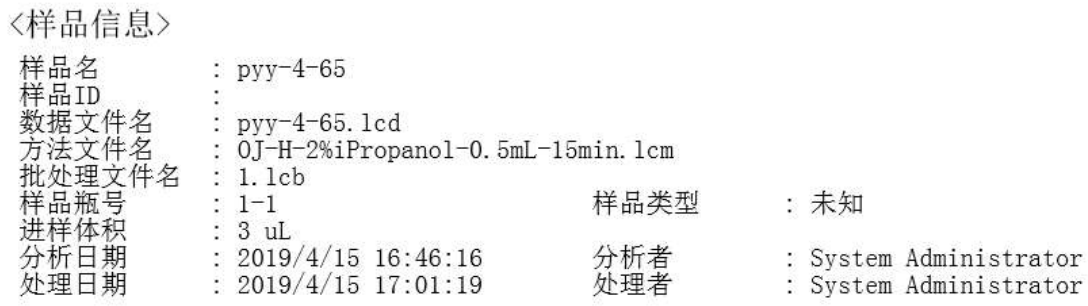

〈色谱图〉

$\mathrm{mAU}$

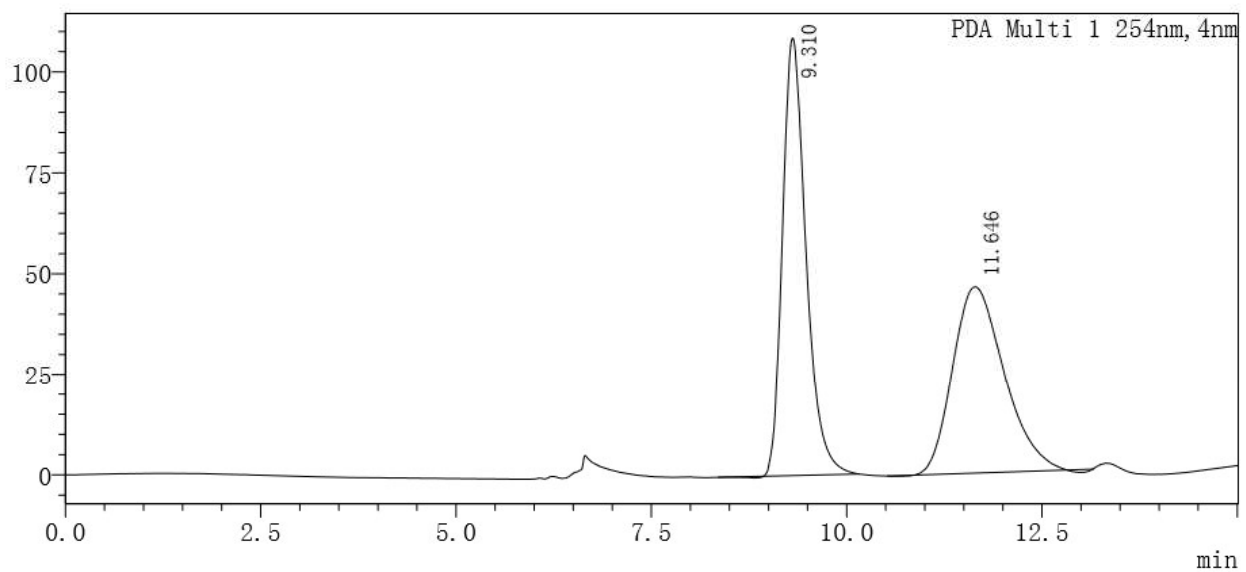

〈峰表〉

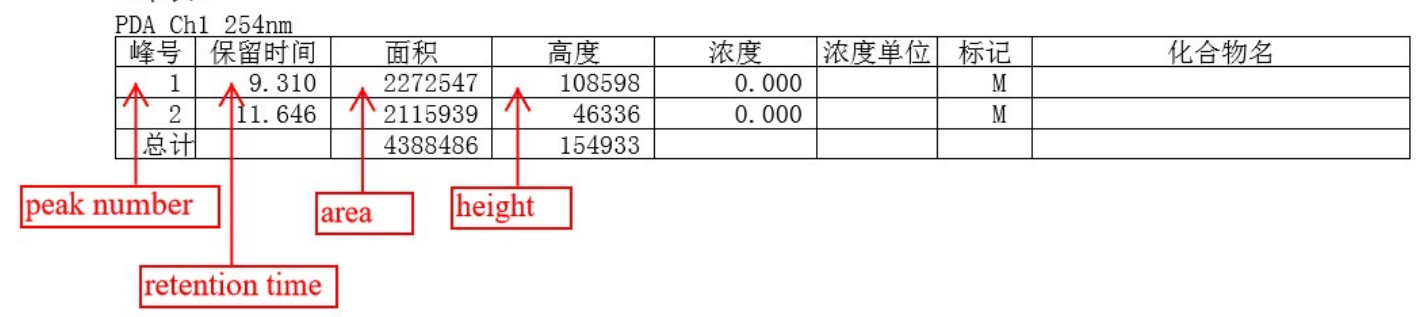




\section{SHIMADZU 分析报告}

〈样品信息〉

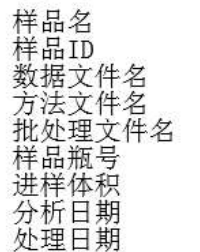

: pyy-4-94

pyy-4-94. lcd

OJ-H-2\%iPropanol-0. 5mL-15min. $1 \mathrm{~cm}$

$1.1 \mathrm{cb}$

$1-2$

2019/4/15 17:01:51

样品类型

: 未知

处理日期

: 2019/4/15 17:16:54

分析者

: System Administrator

处理者 : System Administrator

〈色谱图〉

$\mathrm{mAU}$

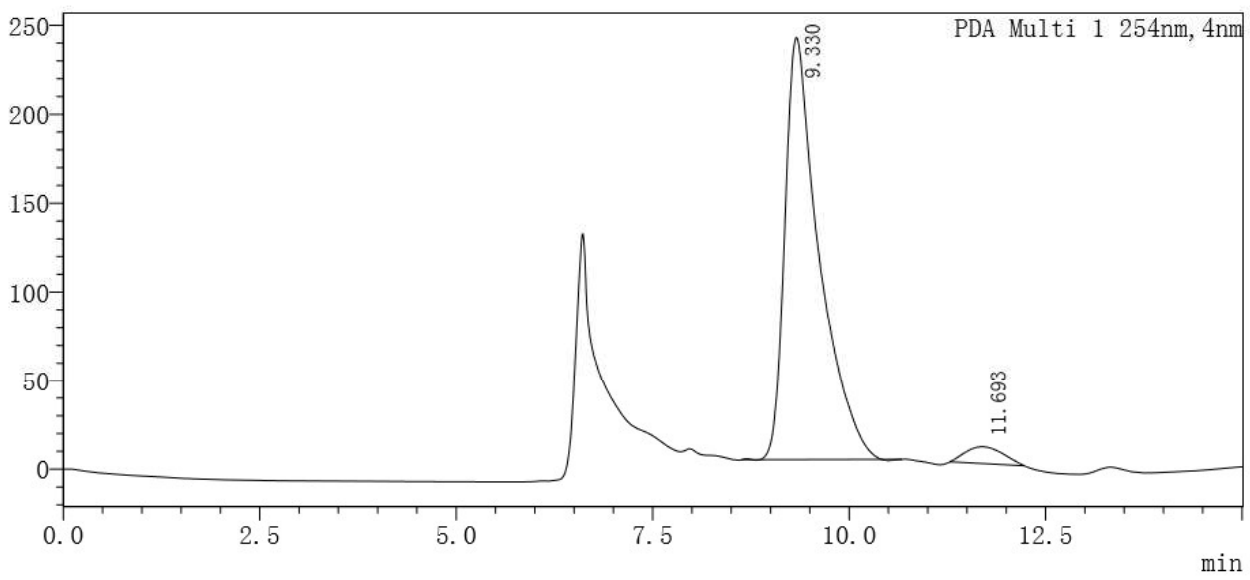

〈峰表〉

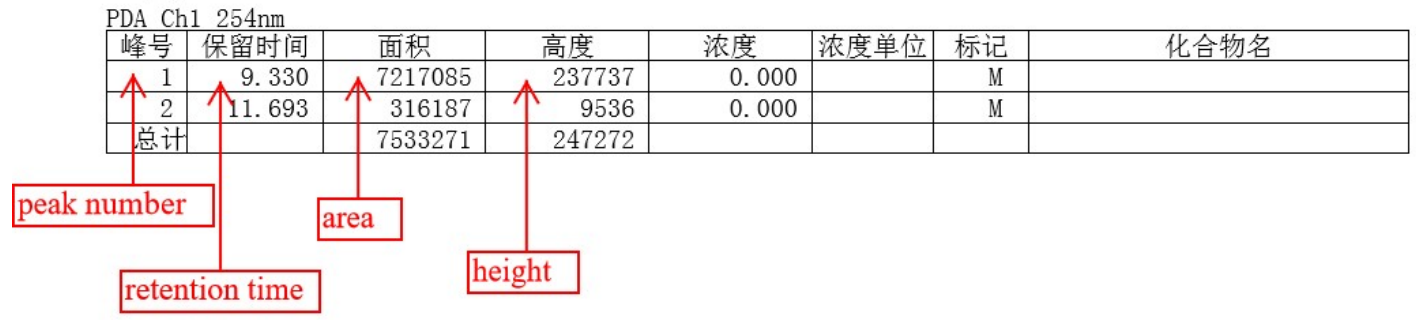


(3aR,8bS)-7-methoxy-2,4,8b-trimethyl-3-phenyl-1,3a,4,8b-tetrahydrocyclopenta[b]indole (8da)

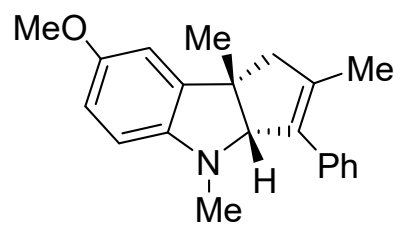

Chemical Formula: $\mathrm{C}_{21} \mathrm{H}_{23} \mathrm{NO}$

Exact Mass: 305.1780

8da was prepared according to general procedure 2.3 using $\mathbf{1 d}$ and $\mathbf{7 a}$ and was purified by silica gel column chromatography (petroleum ether/toluene 2:1-1:99) to obtain $8 \mathrm{da}$ as colorless oil (56\% yield). ${ }^{1} \mathrm{H}$ NMR $\left(400 \mathrm{MHz}, \mathrm{CDCl}_{3}\right) \delta$ 7.39-7.29 (m, 4H), 7.27-7.23 (m, 1H), $6.72(\mathrm{~d}, J=2.6 \mathrm{~Hz}, 1 \mathrm{H}), 6.64(\mathrm{dd}, J=8.4,2.6 \mathrm{~Hz}, 1 \mathrm{H}), 6.29(\mathrm{~d}, J=8.3 \mathrm{~Hz}, 1 \mathrm{H}), 4.40$ (s, 1H), $3.76(\mathrm{~s}, 3 \mathrm{H}), 2.86(\mathrm{~d}, J=16.7 \mathrm{~Hz}, 1 \mathrm{H}), 2.54(\mathrm{~d}, J=16.7 \mathrm{~Hz}, 1 \mathrm{H}), 2.48(\mathrm{~s}, 3 \mathrm{H})$, $1.72(\mathrm{~s}, 3 \mathrm{H}), 1.51(\mathrm{~s}, 3 \mathrm{H}) ;{ }^{13} \mathrm{C} \mathrm{NMR}\left(100 \mathrm{MHz}, \mathrm{CDCl}_{3}\right) \delta 152.6,146.1,139.1,137.8,135.9$, 130.9, 128.5, 128.2, 126.5, 112.0, 110.2, 107.4, 87.8, 56.0, 53.6, 49.7, 37.0, 27.9, 15.1. HRMS: (ESI) calcd for $\mathrm{C}_{21} \mathrm{H}_{24} \mathrm{NO}^{+}[\mathrm{M}+\mathrm{H}]^{+} 306.1866$; found 306.1852 .

The enantiomeric purity was established by HPLC analysis using a chiral column: OJ-H column, $30^{\circ} \mathrm{C}, n$-Hexane/i-Propanol $=95 / 5$ as eluent, $254 \mathrm{~nm}, 1 \mathrm{~mL} / \mathrm{min}$. $\mathrm{tR}=5 \mathrm{~min}$ (major), 7 min (minor).

Optical Rotation: $[\alpha]_{D}{ }^{27}-14.8$ (c $\left.0.4,{ }^{i} \mathrm{PrOH}\right)$ for $89 \%$ ee. 


\section{LabSolutions 分析报告}

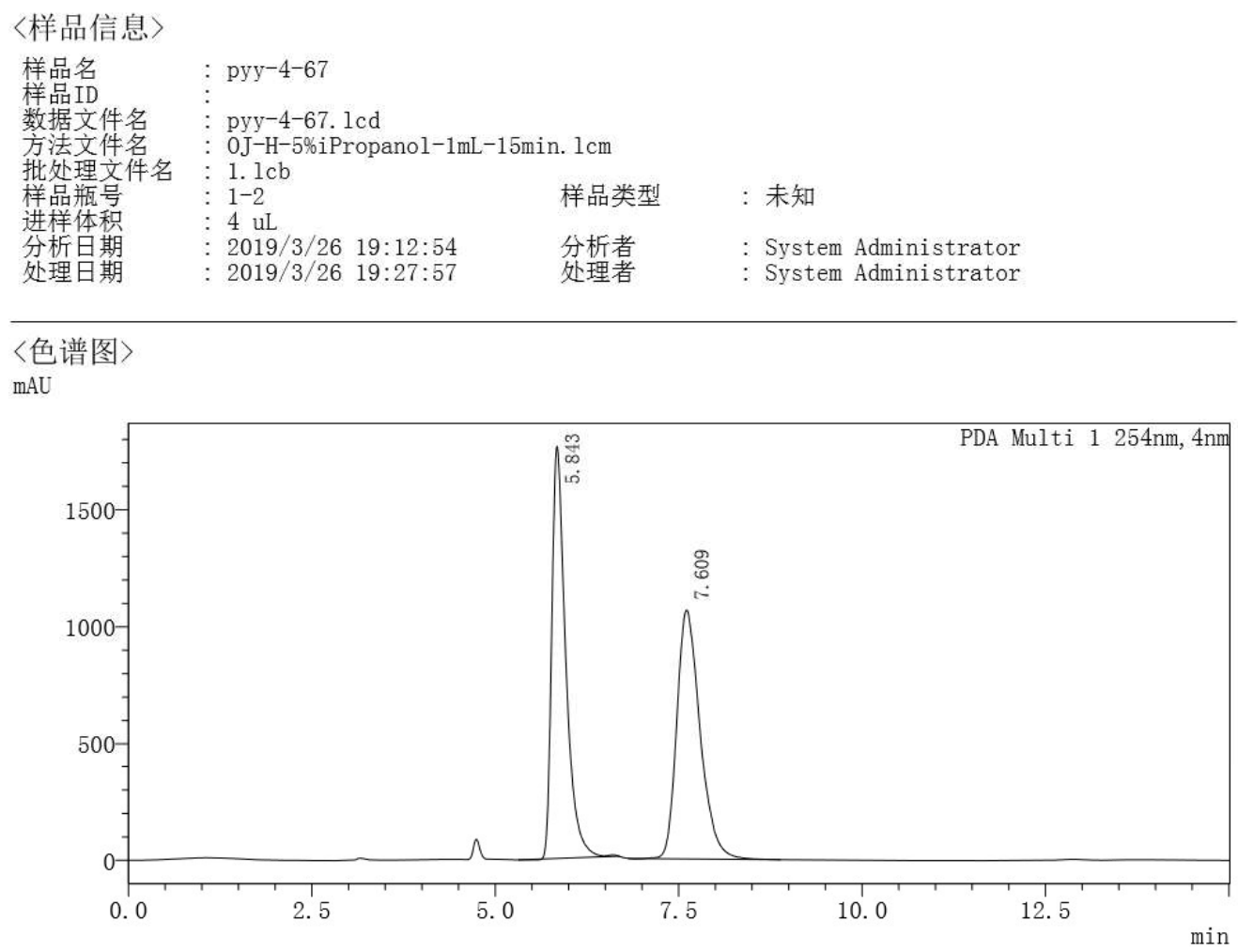

〈峰表〉

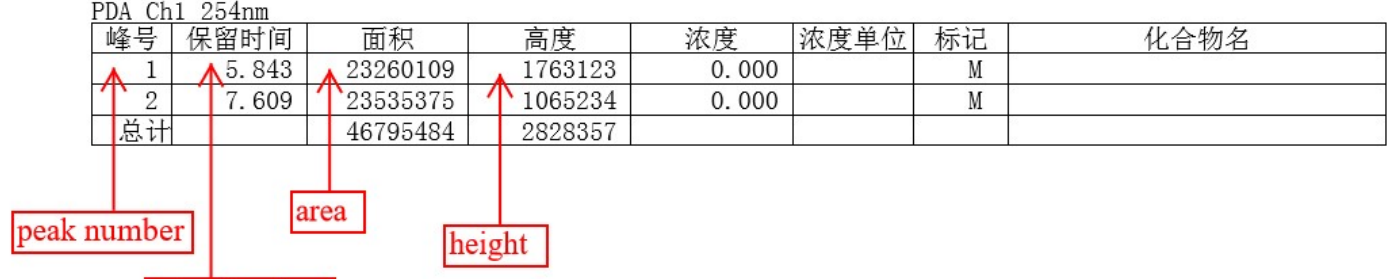

retention time 


\section{LabSolutions 分析报告}

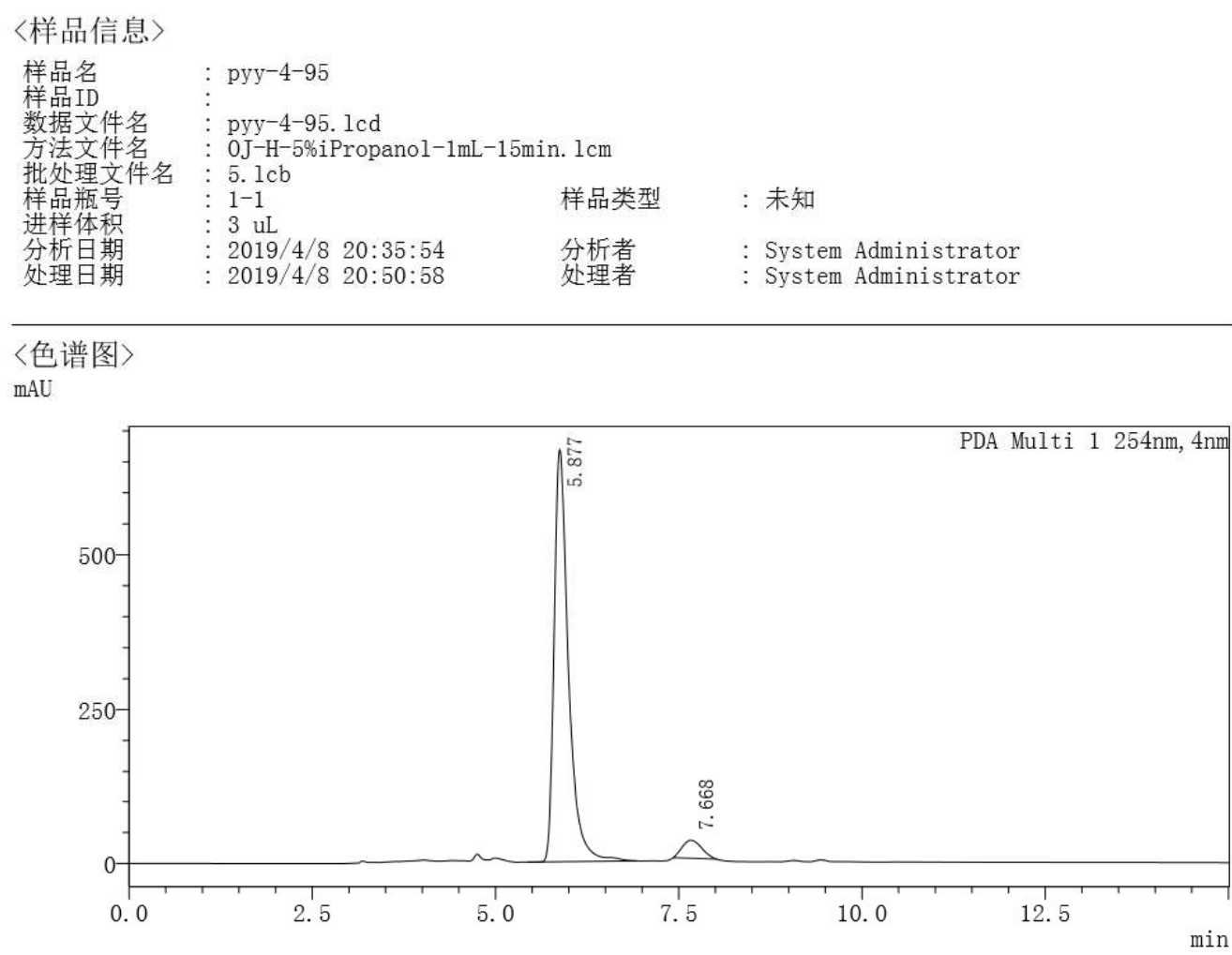

〈峰表〉

\begin{tabular}{|c|c|c|c|c|c|c|c|}
\hline 峰号 & 保留时间 & 面积 & 高度 & 浓度 & 浓度单位 & 标记 & 化合物名 \\
\hline 1 & 5.877 & 9038564 & 667363 & 0.000 & & M & \\
\hline 个2 & 7.668 & 540002 & 个 29267 & 0.000 & & M & \\
\hline 总计 & & 9578566 & 696630 & & & & \\
\hline numbe & & & & & & & \\
\hline
\end{tabular}

retention time 
(3aR,8bS)-2,4,7,8b-tetramethyl-3-phenyl-1,3a,4,8b-tetrahydrocyclopenta[b]indole (8ea)

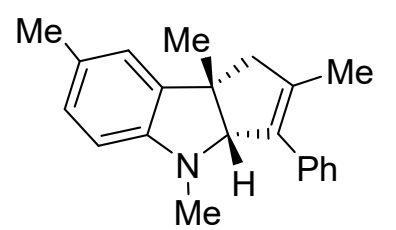

Chemical Formula: $\mathrm{C}_{21} \mathrm{H}_{23} \mathrm{~N}$

Exact Mass: 289.1830

8ea was prepared according to general procedure 2.3 using $1 \mathrm{e}$ and $7 \mathrm{a}$ and was purified by silica gel column chromatography (petroleum ether/toluene 2:1-1:99) to obtain 8ea as colorless oil (48\% yield). ${ }^{1} \mathrm{H}$ NMR $\left(400 \mathrm{MHz}, \mathrm{CDCl}_{3}\right) \delta$ 7.36-7.28 $(\mathrm{m}, 4 \mathrm{H}), 7.25-7.22(\mathrm{~m}$, 1H), 6.91-6.83 (m, 2H), 6.25 (d, J = 7.7 Hz, 1H), $4.43(\mathrm{~s}, 1 \mathrm{H}), 2.86(\mathrm{~d}, J=16.7 \mathrm{~Hz}, 1 \mathrm{H})$, $2.55(\mathrm{~d}, J=16.8 \mathrm{~Hz}, 1 \mathrm{H}), 2.48(\mathrm{~s}, 3 \mathrm{H}), 2.26(\mathrm{~s}, 3 \mathrm{H}), 1.69(\mathrm{~s}, 3 \mathrm{H}), 1.49(\mathrm{~s}, 3 \mathrm{H}) ;{ }^{13} \mathrm{C}$ NMR $\left(101 \mathrm{MHz}, \mathrm{CDCl}_{3}\right) \delta 149.2,138.0,137.9,135.9,128.5,128.1,128.0,126.4,126.2,123.4$, 106.7, 87.3, 53.8, 49.5, 36.0, 29.7, 28.0, 15.0; HRMS: (ESI) calcd for $\mathrm{C}_{21} \mathrm{H}_{24} \mathrm{~N}^{+}[\mathrm{M}+\mathrm{H}]^{+}$ 290.1903 ; found 290.1892 .

The enantiomeric purity was established by HPLC analysis using a chiral column: OJ-H column, $30{ }^{\circ} \mathrm{C}, n$-Hexane/i-Propanol $=95 / 5$ as eluent, $254 \mathrm{~nm}, 1 \mathrm{~mL} / \mathrm{min}$. tR $=4 \mathrm{~min}$ (major), 9 min (minor).

Optical Rotation: [a] ${ }^{26}-100.6$ (c $\left.0.4,{ }^{i} \mathrm{PrOH}\right)$ for $93 \%$ ee. 


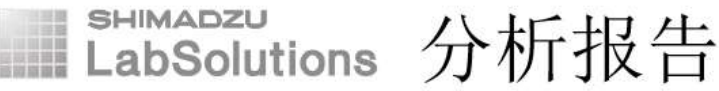

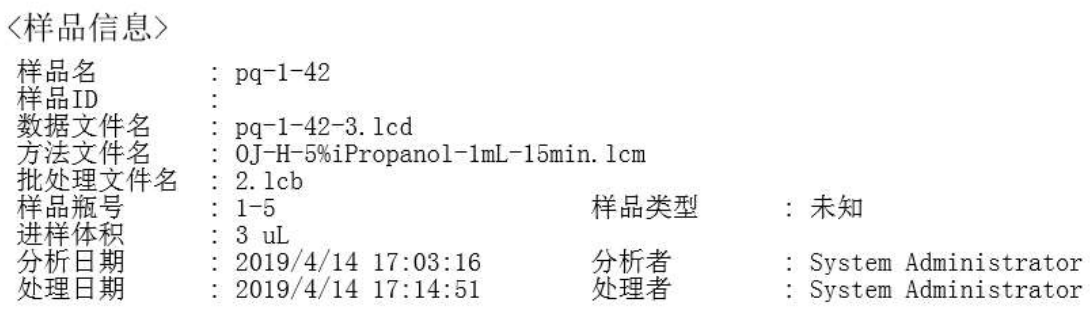

〈色谱图〉

$\mathrm{mAU}$

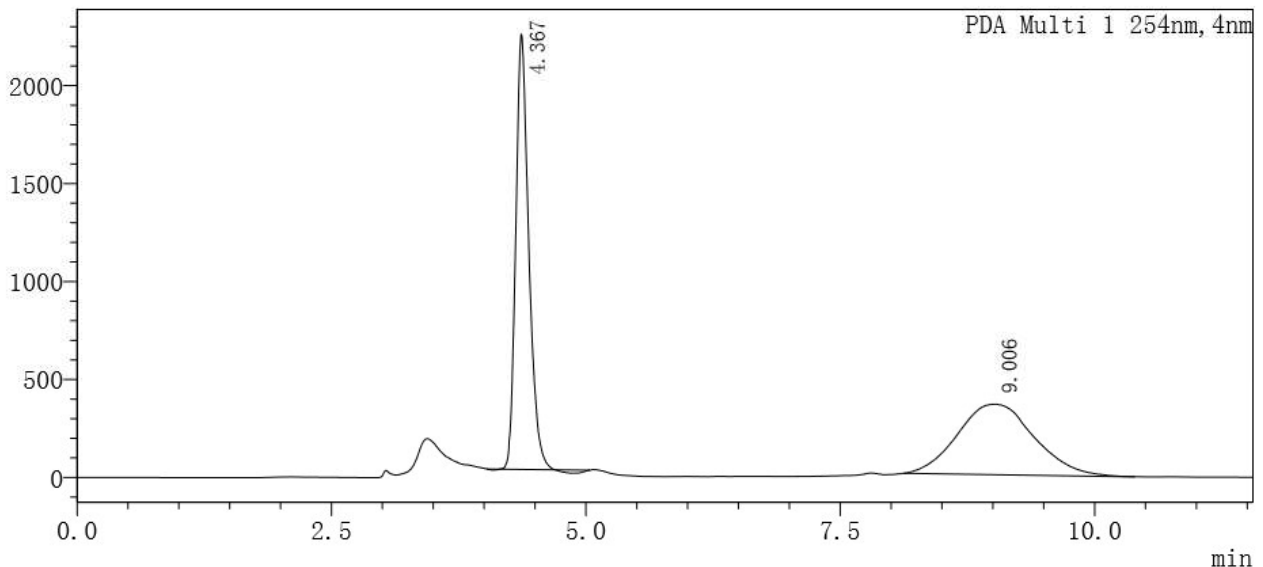

〈峰表〉

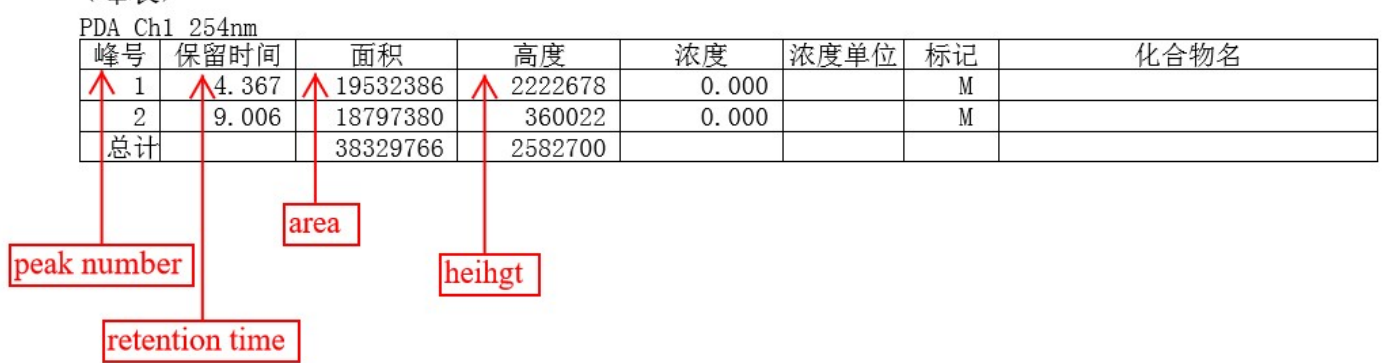


11 Stmangzu

〈样品信息〉

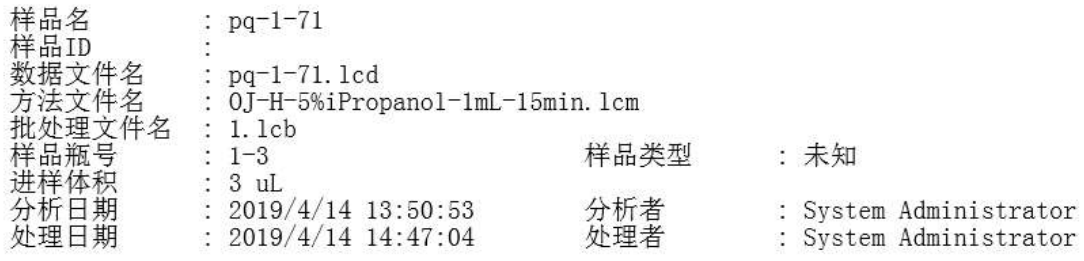

〈色谱图〉

$\mathrm{mAU}$

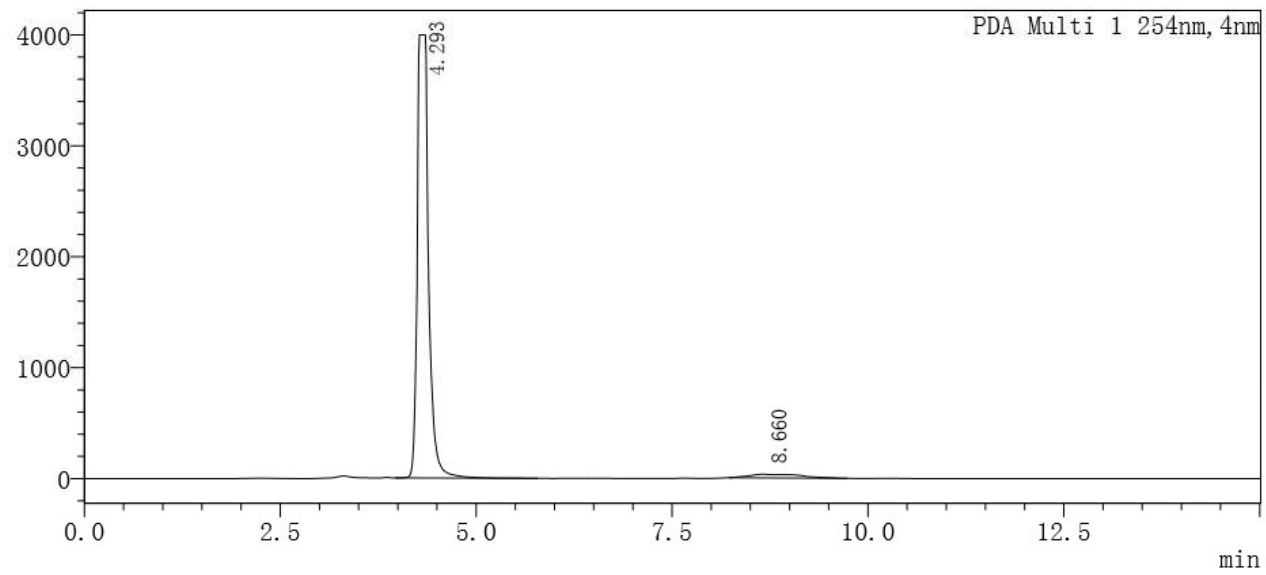

〈峰表〉

\begin{tabular}{|c|c|c|c|c|c|c|c|}
\hline 峰号 & 保留时间 & 面积 & 高度 & 浓度 & 浓度单位 & 标记 & 化合物名 \\
\hline 1 & 4.293 & 40254266 & 3994618 & 0.000 & & M & \\
\hline 2 & 8.660 & 1502509 & 31968 & 0.000 & & M & \\
\hline 总计 & & 41756775 & 4026586 & & & & \\
\hline
\end{tabular}

retention time 
(3aR,8bS)-2,4,6,8b-tetramethyl-3-phenyl-1,3a,4,8b-tetrahydrocyclopenta[b]indole (8fa)

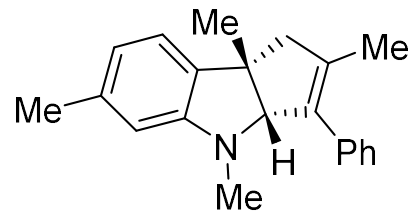

Chemical Formula: $\mathrm{C}_{21} \mathrm{H}_{23} \mathrm{~N}$

Exact Mass: 289.1830

8fa was prepared according to general procedure 2.3 using $1 \mathbf{f}$ and $7 \mathrm{a}$ and was purified by silica gel column chromatography (petroleum ether/toluene 2:1-1:99) to obtain $\mathbf{8 f a}$ as colorless oil (69\% yield). ${ }^{1} \mathrm{H}$ NMR $\left(400 \mathrm{MHz}, \mathrm{CDCl}_{3}\right) \delta$ 7.38-7.31 (m, 2H), 7.30-7.23 (m, 3H), 6.95 (d, J = 7.3 Hz, 1H), $6.46(\mathrm{~d}, J=7.3 \mathrm{~Hz}, 1 \mathrm{H}), 6.13(\mathrm{~s}, 1 \mathrm{H}), 4.49(\mathrm{~s}, 1 \mathrm{H}), 2.84(\mathrm{~d}$, $J=16.7 \mathrm{~Hz}, 1 \mathrm{H}), 2.57(\mathrm{~d}, J=16.8 \mathrm{~Hz}, 1 \mathrm{H}), 2.47(\mathrm{~s}, 3 \mathrm{H}), 2.26(\mathrm{~s}, 3 \mathrm{H}), 1.67(\mathrm{~s}, 3 \mathrm{H}), 1.48$ (s, 3H); ${ }^{13} \mathrm{C}$ NMR $\left(100 \mathrm{MHz}, \mathrm{CDCl}_{3}\right) \delta 151.2,138.0,137.9,137.6,135.8,134.9,128.5$, 128.1, 126.4, 122.2, 117.3, 107.1, 87.0, 53.9, 49.1, 35.1, 28.1, 21.7, 15.0; HRMS: (ESI) calcd for $\mathrm{C}_{21} \mathrm{H}_{24} \mathrm{~N}^{+}[\mathrm{M}+\mathrm{H}]^{+} 290.1908$; found 290.1903 .

The enantiomeric purity was established by HPLC analysis using a chiral column: OJ-H column, $30{ }^{\circ} \mathrm{C}, n$-Hexane/i-Propanol $=95 / 5$ as eluent, $254 \mathrm{~nm}, 1 \mathrm{~mL} / \mathrm{min}$. $\mathrm{tR}=4 \mathrm{~min}$ (major), 7 min (minor).

Optical Rotation: $[\alpha]_{D}^{28}-24.5$ (c 0.4 , $\left.\mathrm{PrOH}\right)$ for $92 \%$ ee. 


\section{LabSolutions 分析报告}

〈样品信息〉

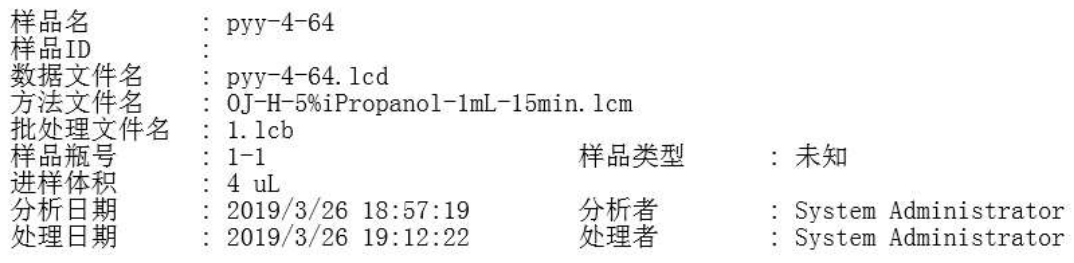

〈色谱图〉

$\mathrm{mAU}$

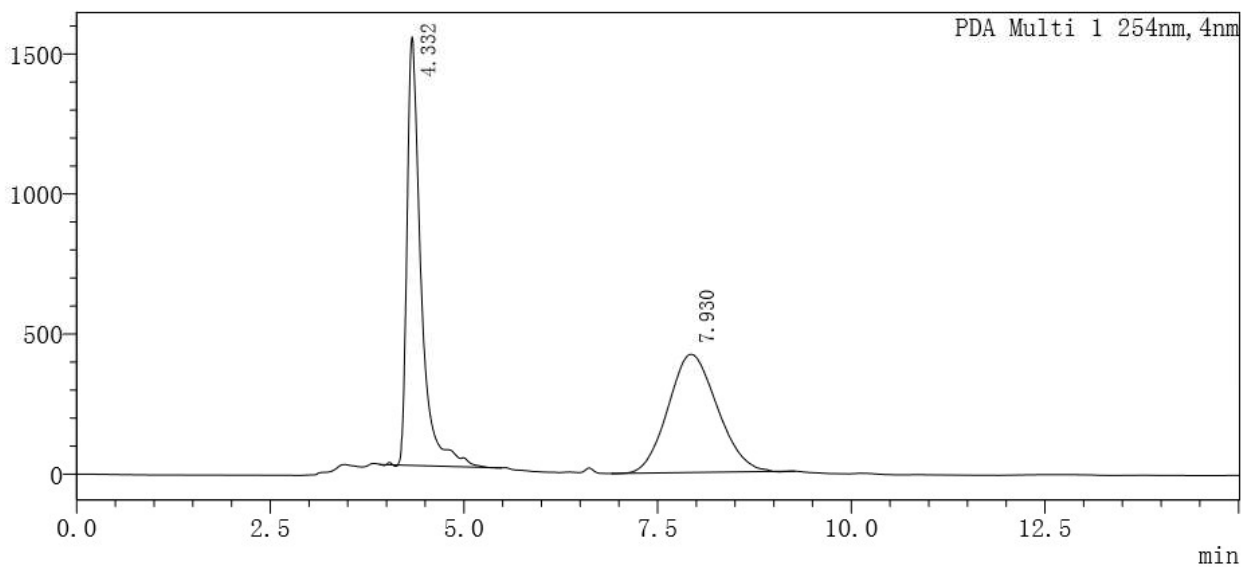

〈峰表〉

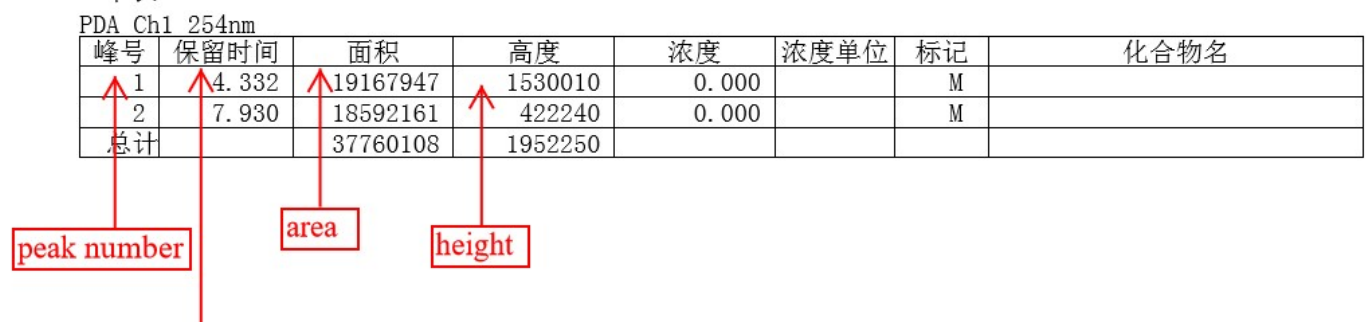

retention time 


\section{in SHMABDZu}

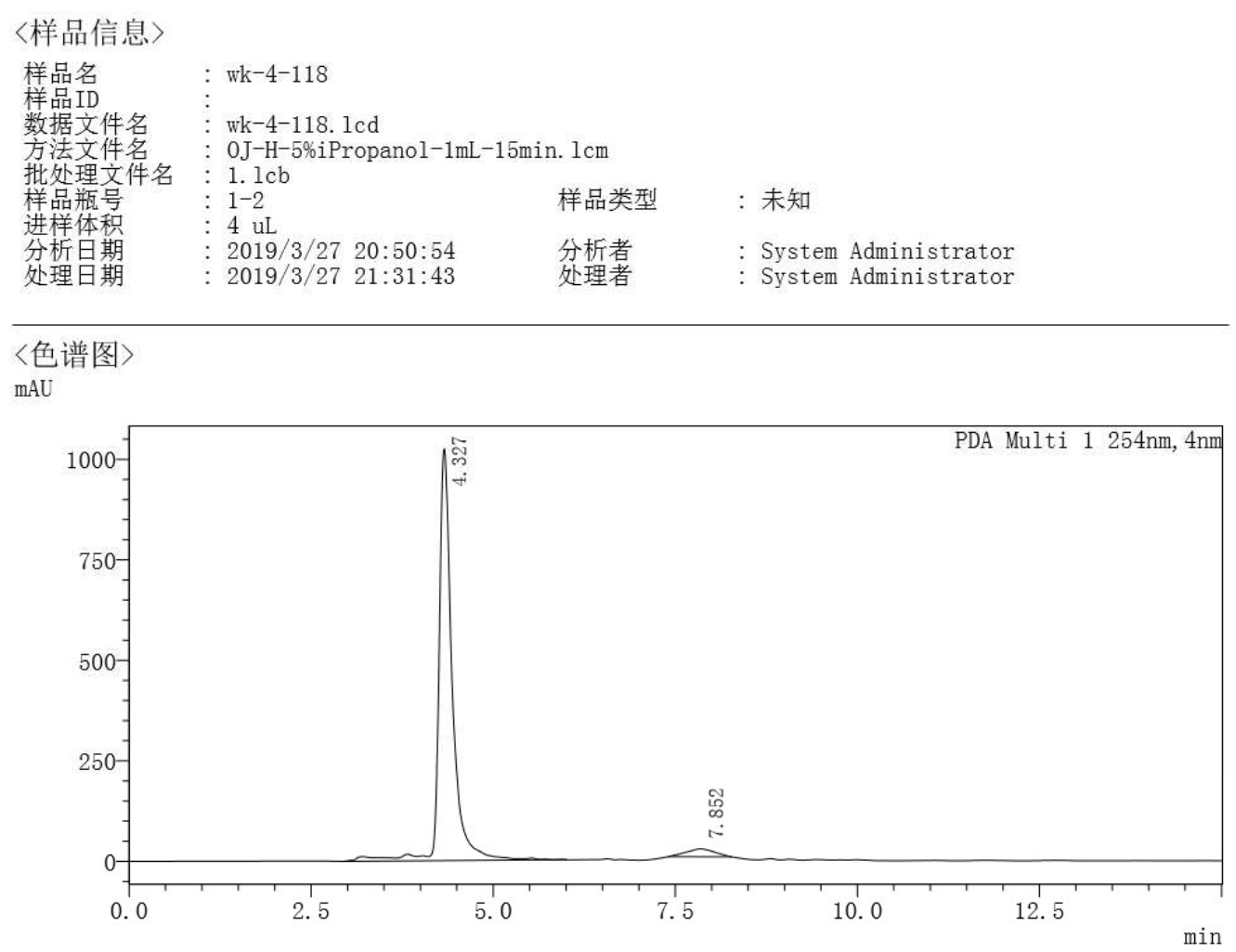

〈峰表〉

\begin{tabular}{|c|c|c|c|c|c|c|c|}
\hline 峰号 & 保留时间 & 面积 & 高度 & 浓度 & 浓度单位 & 标记 & 化合物名 \\
\hline 1 & 4.327 & 13061126 & 1024581 & 0.000 & & M & \\
\hline 72 & 7.852 & 我 535622 & 19980 & 0.000 & & M & \\
\hline 总计 & & 13596748 & 1044561 & & & & \\
\hline number & & & height & & & & \\
\hline
\end{tabular}

retention time 
(3aR,8bS)-6-methoxy-2,4,8b-trimethyl-3-phenyl-1,3a,4,8b-tetrahydrocyclopenta[b]indole (8ga)

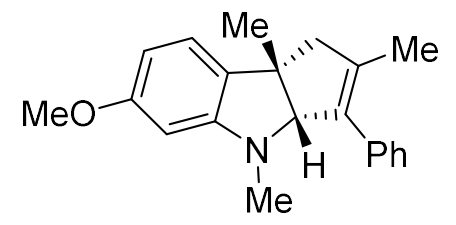

Chemical Formula: $\mathrm{C}_{21} \mathrm{H}_{23} \mathrm{NO}$

Exact Mass: 305.1780

8ga was prepared according to general procedure 2.3 using $\mathbf{1 g}$ and $7 \mathrm{a}$ and was purified by silica gel column chromatography (petroleum ether/toluene 2:1-1:99) to obtain $8 \mathrm{ga}$ as colorless oil (60\% yield). ${ }^{1} \mathrm{H}$ NMR $\left(400 \mathrm{MHz}, \mathrm{CDCl}_{3}\right)$ ס 7.44-7.18 (m, 5H), 6.94 (d, J = 8.0 $\mathrm{Hz}, 1 \mathrm{H}), 6.17(\mathrm{dd}, J=8.0,2.2 \mathrm{~Hz}, 1 \mathrm{H}), 5.88(\mathrm{~d}, J=2.1 \mathrm{~Hz}, 1 \mathrm{H}), 4.52(\mathrm{~s}, 1 \mathrm{H}), 3.75(\mathrm{~s}, 3 \mathrm{H})$, $2.84(\mathrm{~d}, J=16.7 \mathrm{~Hz}, 1 \mathrm{H}), 2.56(\mathrm{~d}, J=16.8 \mathrm{~Hz}, 1 \mathrm{H}), 2.46(\mathrm{~s}, 3 \mathrm{H}), 1.68(\mathrm{~s}, 3 \mathrm{H}), 1.47(\mathrm{~s}$, $3 \mathrm{H}) ;{ }^{13} \mathrm{C} \mathrm{NMR}\left(100 \mathrm{MHz}, \mathrm{CDCl}_{3}\right) \delta 160.4,152.3,138.0,137.9,135.8,130.4,128.5,128.2$, 126.5, 122.5, 100.7, 93.2, 87.3, 55.2, 53.9, 48.8, 34.8, 28.1, 15.0; HRMS: (ESI) calcd for $\mathrm{C}_{21} \mathrm{H}_{24} \mathrm{NO}^{+}[\mathrm{M}+\mathrm{H}]^{+}$306.1863; found 306.1852.

The enantiomeric purity was established by HPLC analysis using a chiral column: OJ-H column, $30^{\circ} \mathrm{C}, n$-Hexane/i-Propanol $=90 / 10$ as eluent, $254 \mathrm{~nm}, 1 \mathrm{~mL} / \mathrm{min}$. $\mathrm{tR}=5 \mathrm{~min}$ (major), 12 min (minor).

Optical Rotation: $[\alpha]^{27}-37.6$ (c 0.4 , $\mathrm{PrOH}$ ) for $96 \%$ ee. 


\section{Lin SHMADZu}

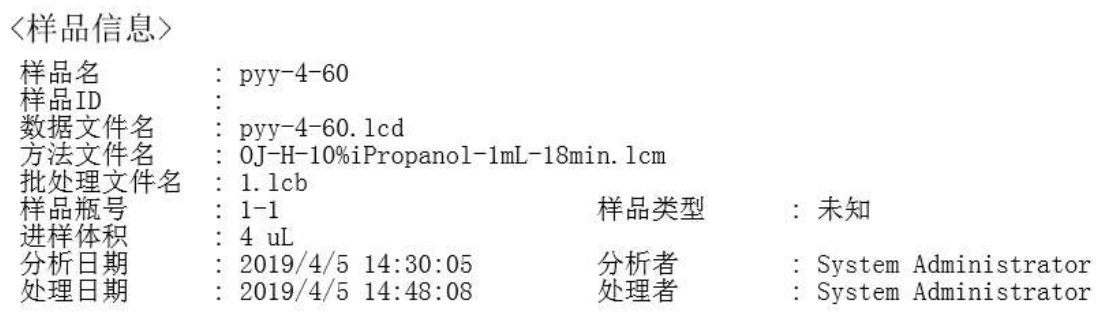

〈色谱图〉

$\mathrm{mAU}$

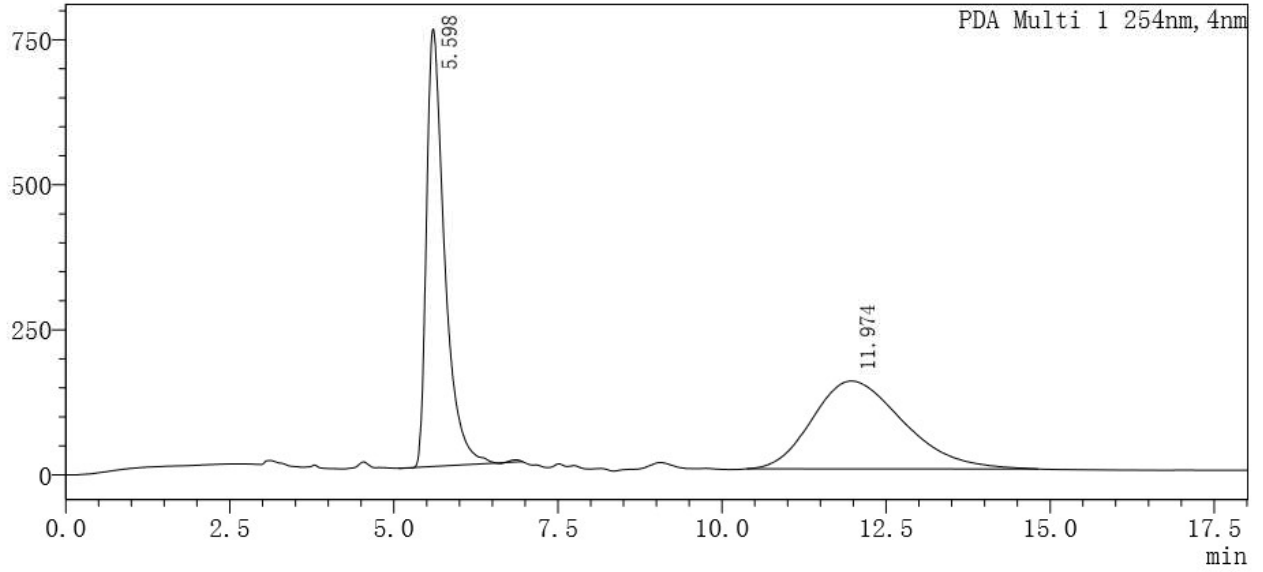

〈峰表〉

PDA Ch1 254nm

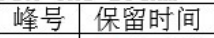

\begin{tabular}{|r|r|}
\hline 1 & 峰留时间 \\
\hline 1 & 5.598 \\
\hline
\end{tabular}

\begin{tabular}{ll|r} 
& 1 & 5.598 \\
\hline & 2 & 11.974
\end{tabular}

\begin{tabular}{|r|r|r|r|}
\hline 1 & 5.598 & 14501658 \\
\hline & 2 & 11.974 & 14628812 \\
\hline 总计 & & 29130470
\end{tabular}

\section{height}

peak number

retention time 


\section{LabSolutions 分析报告}

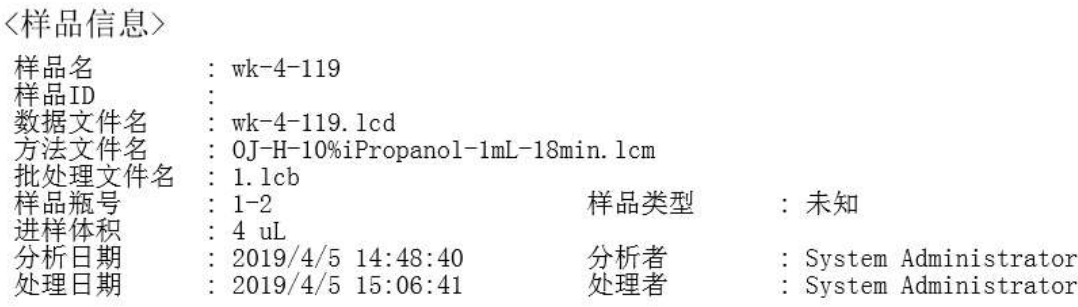

样品ID

数据文件名： : wk-4-119

批处理文件名 : $1.1 \mathrm{cb}$

样品瓶号

进样体积

分析日期

2019/4/5 14:48:40

: 2019/4/5 15:06:41

样品类型

: 未知

处理日期

分析者

: System Administrator

处理者 : System Administrator

〈色谱图〉

$\mathrm{mAU}$

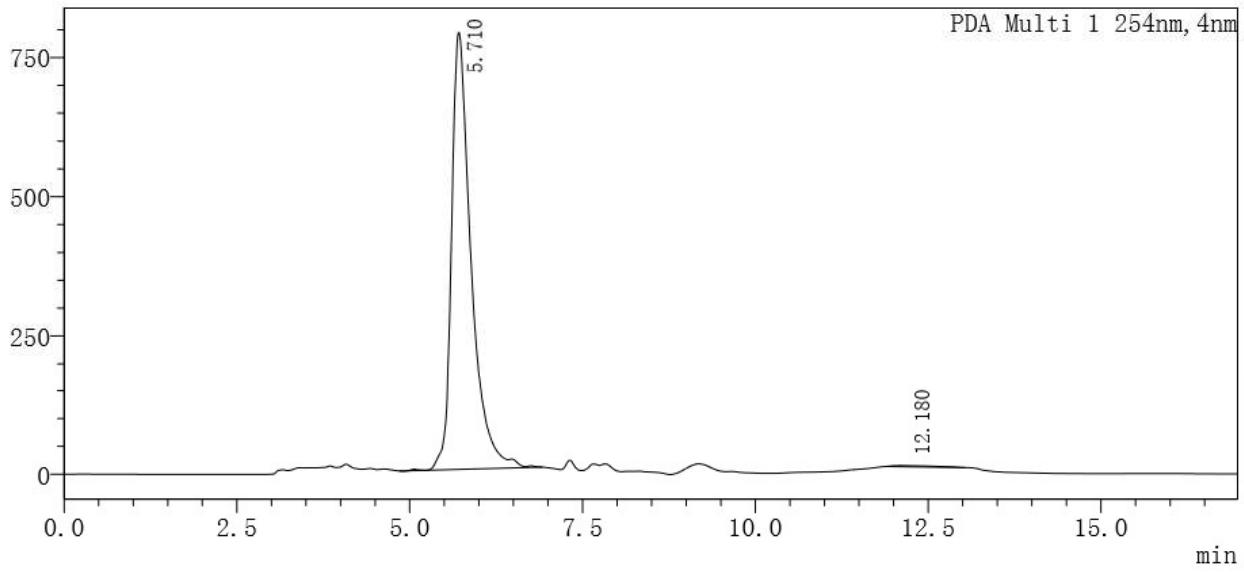

〈峰表〉

\begin{tabular}{|c|c|c|c|c|c|c|c|}
\hline 峰号 & 保留时间 & 面积 & 高度 & 浓度 & 浓度单位 & 标记 & 化合物名 \\
\hline 1 & 5.710 & 15547981 & 786329 & 0.000 & & M & \\
\hline 2 & A2. 180 & 95041 & 2286 & 0.000 & & M & \\
\hline 总计 & & 15643023 & 788614 & & & & \\
\hline $\mathrm{umbe}$ & & & height & & & & \\
\hline
\end{tabular}

retention time 
(3aR,8bS)-6-chloro-2,4,8b-trimethyl-3-phenyl-1,3a,4,8b-tetrahydrocyclopenta[b]indole (8ha)

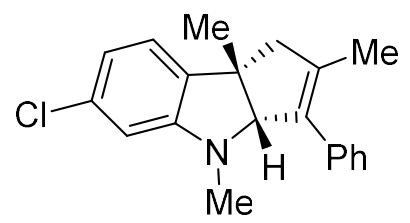

Chemical Formula: $\mathrm{C}_{20} \mathrm{H}_{20} \mathrm{CIN}$

Exact Mass: 309.1284

8ha was prepared according to general procedure 2.3 using $1 \mathrm{~h}$ and $7 \mathrm{a}$ and was purified by silica gel column chromatography (petroleum ether/toluene 2:1-1:99) to obtain 8 ha as colorless oil (57\% yield). ${ }^{1} \mathrm{H}$ NMR $\left(400 \mathrm{MHz}, \mathrm{CDCl}_{3}\right) \delta$ 7.40-7.34 (m, 2H), 7.30-7.21(m, 3H), $6.92(\mathrm{~d}, J=7.7 \mathrm{~Hz}, 1 \mathrm{H}), 6.56(\mathrm{dd}, J=7.7,1.8 \mathrm{~Hz}, 1 \mathrm{H}), 6.21(\mathrm{~d}, J=1.7 \mathrm{~Hz}, 1 \mathrm{H}), 4.56$ (s, 1H), $2.82(\mathrm{~d}, J=16.8 \mathrm{~Hz}, 1 \mathrm{H}), 2.59(\mathrm{~d}, J=16.8 \mathrm{~Hz}, 1 \mathrm{H}), 2.44(\mathrm{~s}, 3 \mathrm{H}), 1.68(\mathrm{~s}, 3 \mathrm{H})$, 1.47 (s, 3H); ${ }^{13} \mathrm{C}$ NMR (100 MHz, $\left.\mathrm{CDCl}_{3}\right) \delta 151.9,138.0,137.6,136.3,135.7,133.4$, 128.4, 128.3, 126.6, 123.1, 116.0, 105.9, 86.7, 53.7, 49.0, 34.4, 27.7, 14.9; HRMS: (ESI) calcd for $\mathrm{C}_{20} \mathrm{H}_{21} \mathrm{CIN}^{+}[\mathrm{M}+\mathrm{H}]^{+} 310.1350$; found 310.1357 .

The enantiomeric purity was established by HPLC analysis using a chiral column: OJ-H column, $30{ }^{\circ} \mathrm{C}, n$-Hexane/i-Propanol $=95 / 5$ as eluent, $254 \mathrm{~nm}, 1 \mathrm{~mL} / \mathrm{min}$. $\mathrm{tR}=5 \mathrm{~min}$ (major), 13 min (minor).

Optical Rotation: $[\alpha]^{23}-52.5$ (c 0.4 , $\left.\mathrm{PrOH}\right)$ for $90 \%$ ee. 


\section{LabSolutions 分析报告}
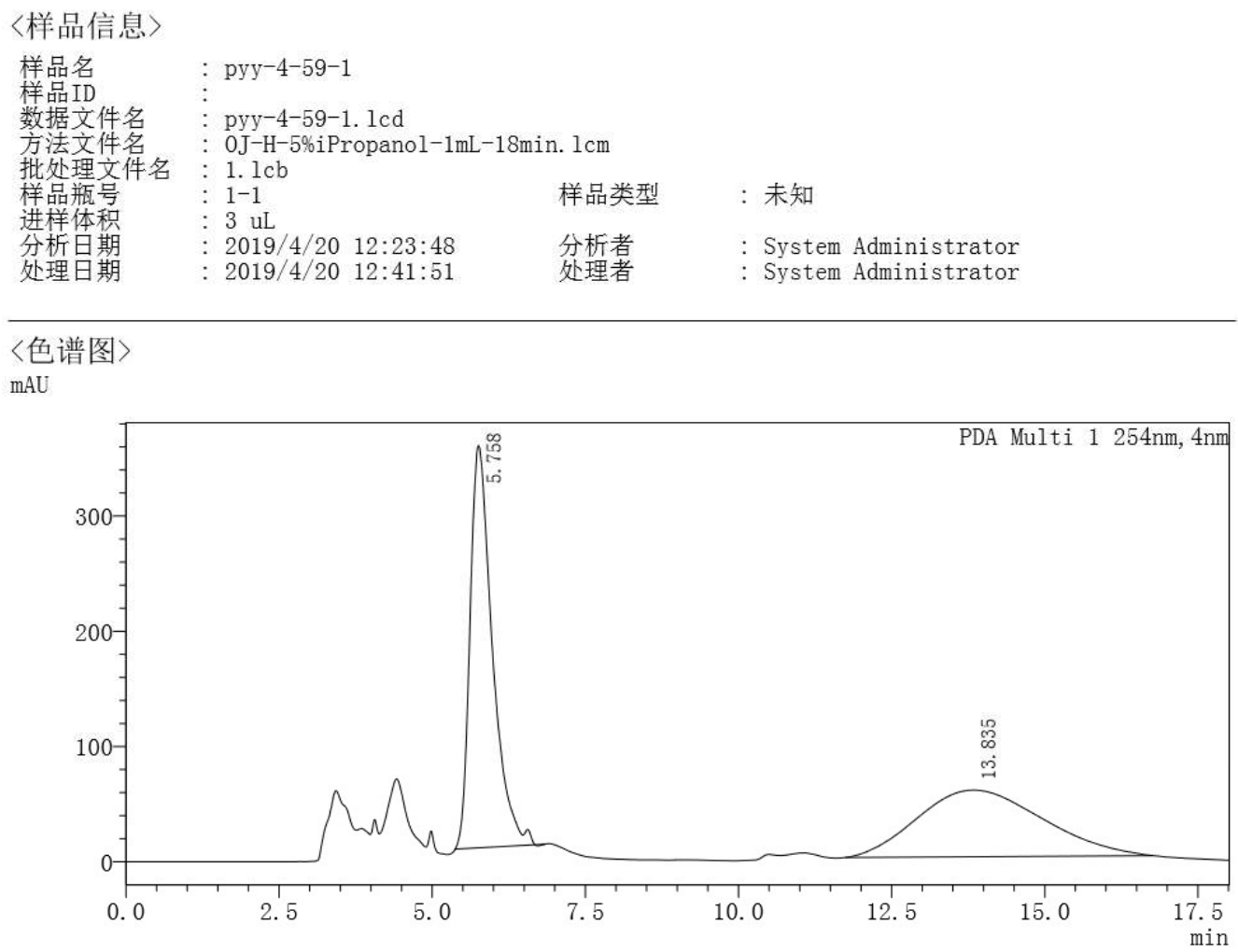

〈峰表〉

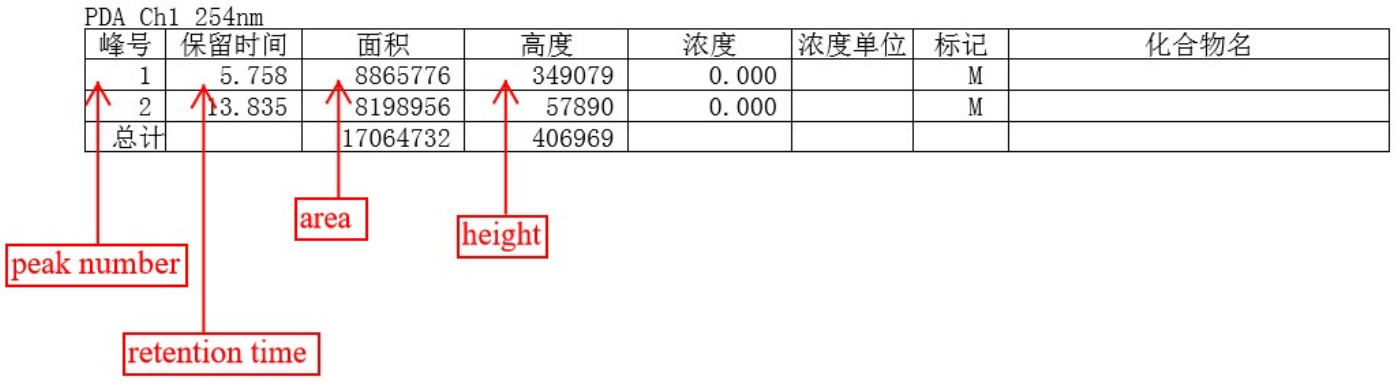




\section{in LabSolutions 分析报告}
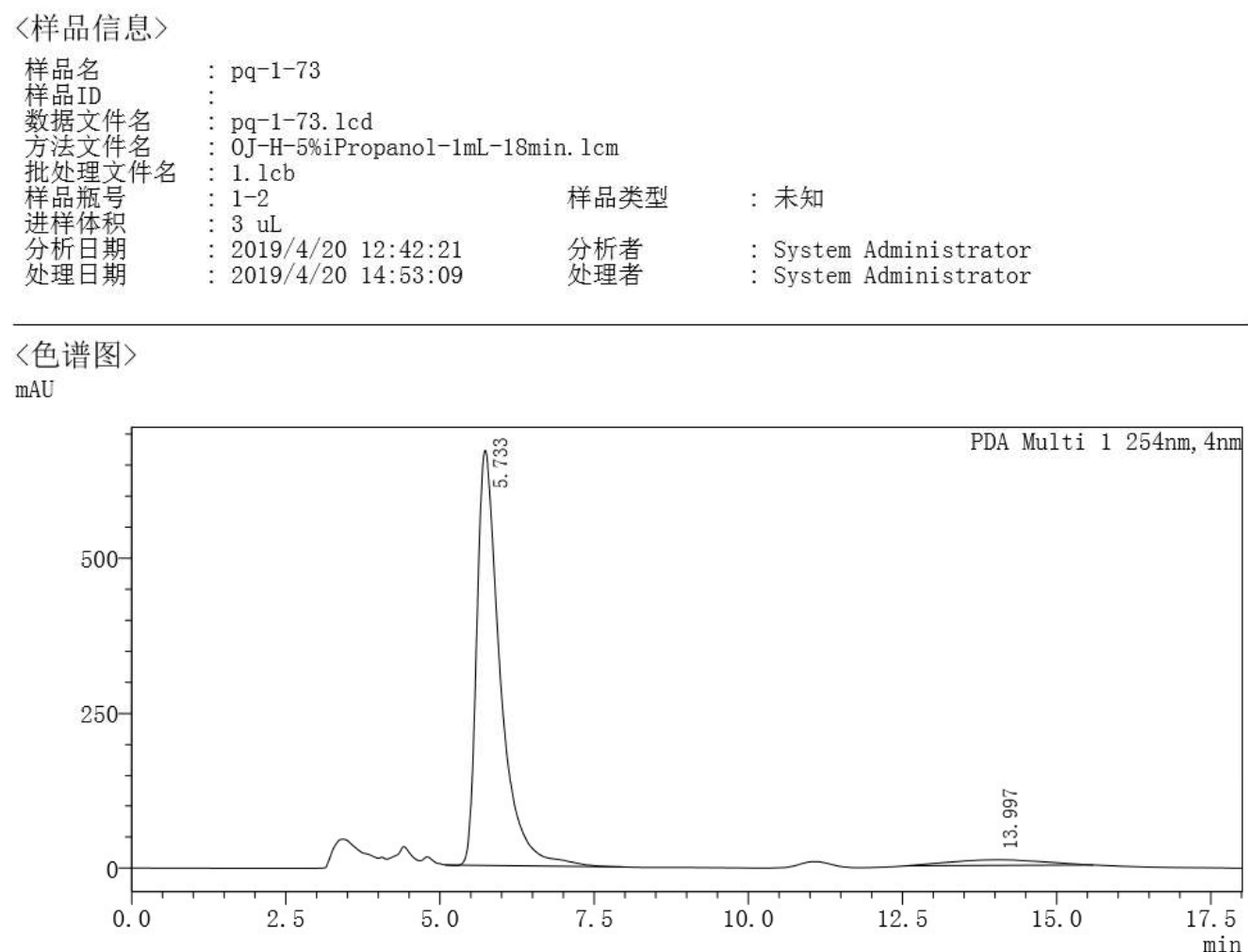

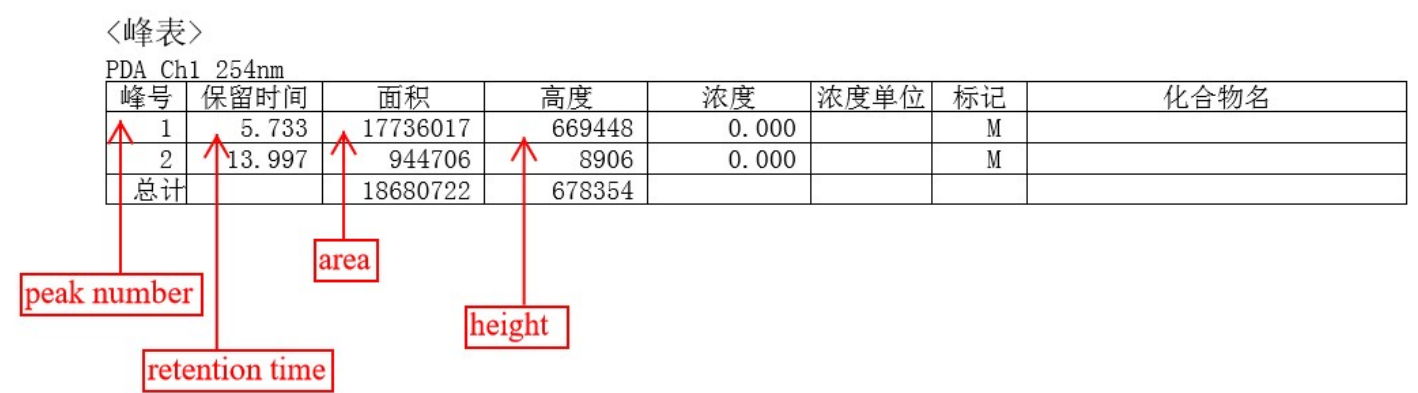


(3aR,8bS)-2,4,10b-trimethyl-3-phenyl-1,3a,4,10b-tetrahydrobenzo[f]cyclopenta[b]indole (8ja)

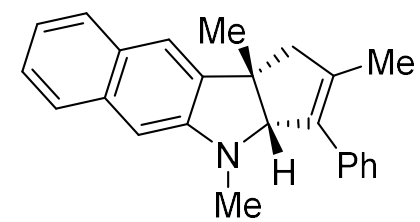

Chemical Formula: $\mathrm{C}_{24} \mathrm{H}_{23} \mathrm{~N}$

Exact Mass: 325.1830

8ja was prepared according to general procedure 2.3 using $3-(\mathrm{N}-$ methylmethacrylamido)naphthalen-2-yl trifluoromethanesulfonate and $\mathbf{7 a}$ and was purified by silica gel column chromatography (petroleum ether/toluene 2:1-1:99) to obtain 8ja as white solid (88\% yield). ${ }^{1} \mathrm{H}$ NMR $\left(400 \mathrm{MHz}, \mathrm{CDCl}_{3}\right) \delta 7.63$ (d, $\left.J=7.6 \mathrm{~Hz}, 1 \mathrm{H}\right), 7.54$ $(\mathrm{d}, J=8.1 \mathrm{~Hz}, 1 \mathrm{H}), 7.43(\mathrm{~s}, 1 \mathrm{H}), 7.38-7.32(\mathrm{~m}, 2 \mathrm{H}), 7.28-7.23(\mathrm{~m}, 4 \mathrm{H}), 7.15-7.08(\mathrm{~m}, 1 \mathrm{H})$, $6.38(\mathrm{~s}, 1 \mathrm{H}), 4.63(\mathrm{~s}, 1 \mathrm{H}), 2.95(\mathrm{~d}, J=16.7 \mathrm{~Hz}, 1 \mathrm{H}), 2.68(\mathrm{~d}, J=16.8 \mathrm{~Hz}, 1 \mathrm{H}), 2.55$ (s, $3 \mathrm{H}), 1.67$ (s, 3H), 1.57 (s, 3H); ${ }^{13} \mathrm{C}$ NMR (100 MHz, $\left.\mathrm{CDCl}_{3}\right) \delta$ 149.5, 141.9, 138.1, 137.8, 136.0, 135.4, 128.4, 128.2, 127.9, 127.5, 126.6, 125.4, 125.3, 121.1, 97.9, 85.9, 53.5, 49.0, 34.0, 29.7, 27.5, 14.9; HRMS: (ESI) calcd for $\mathrm{C}_{24} \mathrm{H}_{24} \mathrm{~N}^{+}[\mathrm{M}+\mathrm{H}]^{+} 326.1903$; found 326.1898 .

The enantiomeric purity was established by HPLC analysis using a chiral column: AD-H column, $30^{\circ} \mathrm{C}, n$-Hexane/i-Propanol $=97 / 3$ as eluent, $254 \mathrm{~nm}, 1 \mathrm{~mL} / \mathrm{min}$. $\mathrm{tR}=3 \mathrm{~min}$ (minor), 4 min (major).

Optical Rotation: $[\alpha]_{D}{ }^{27}-26.1$ (c 0.4 , $\left.{ }^{i} \mathrm{PrOH}\right)$ for $86 \%$ ee. 


\section{In LabSolutions 分析报告}

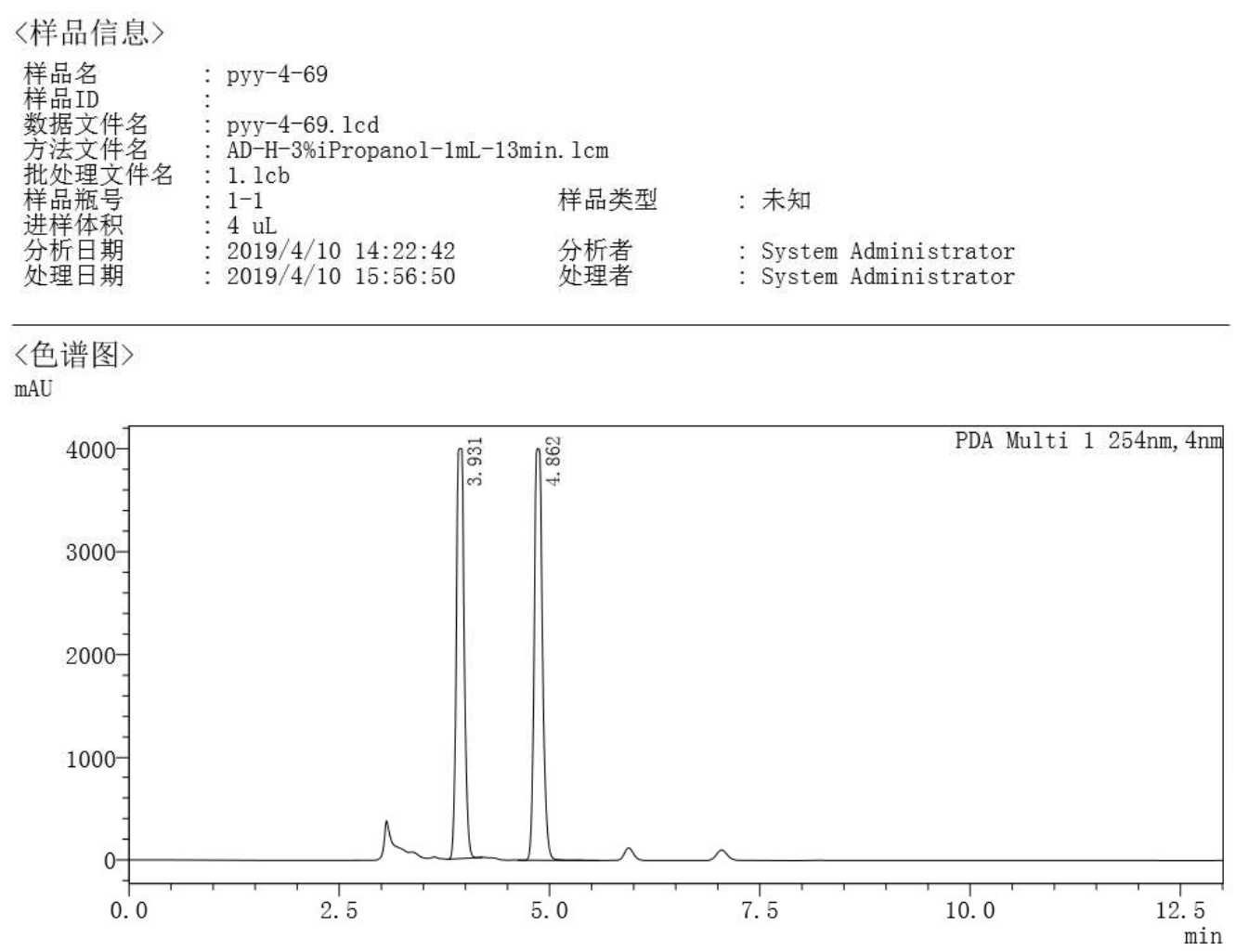

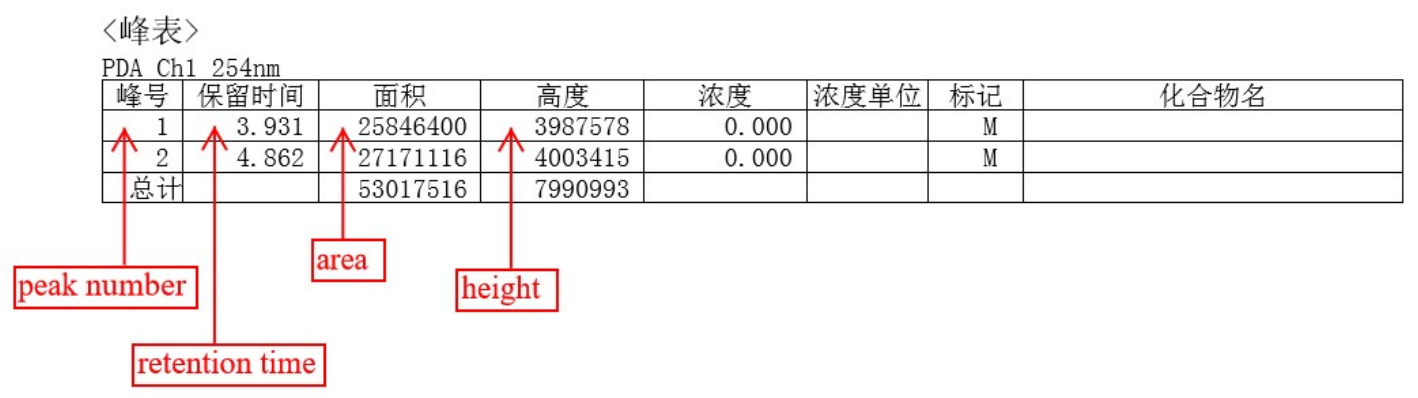




\section{LabSolutions 分析报告}

〈样品信息〉

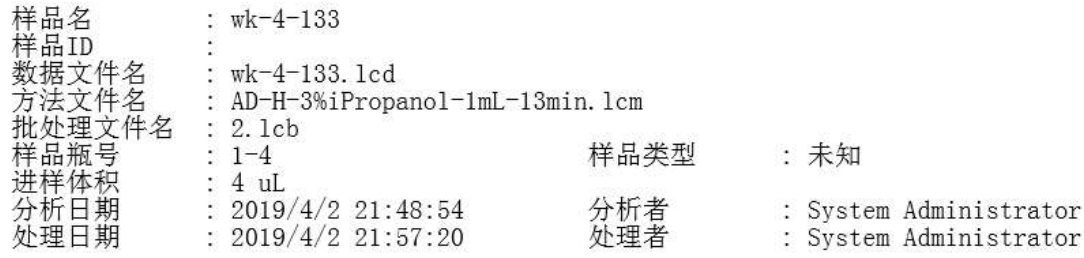

〈色谱图〉

$\mathrm{mAU}$

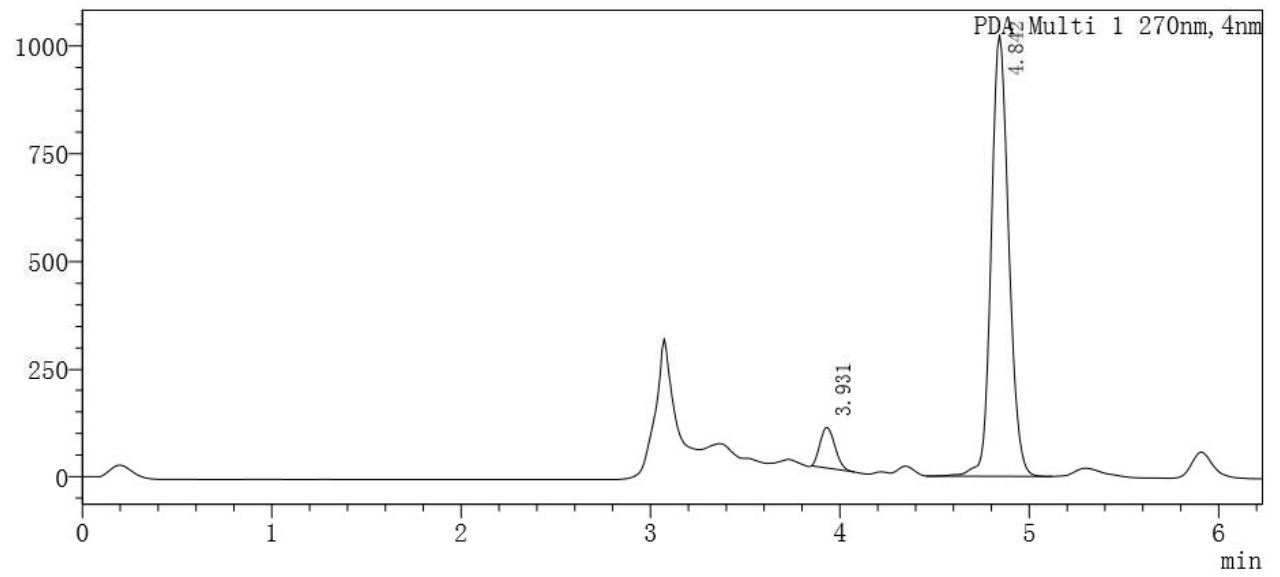

〈峰表〉

PDA Ch1 270nm 峰号 保留时间

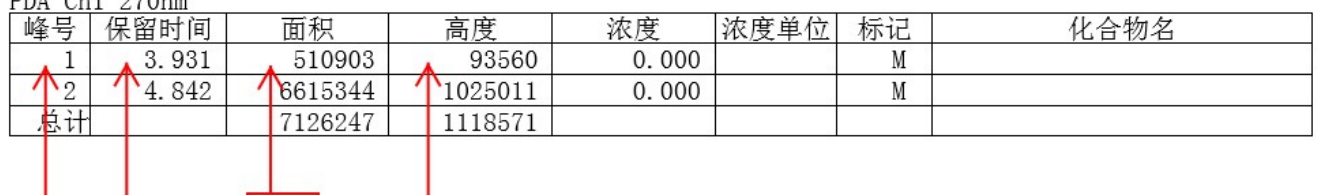


(3aR,8bS)-2,9,10a-trimethyl-8-phenyl-5,6,7a,10a-tetrahydro-4H,10H-

cyclopenta[4,5]pyrrolo[3,2,1-ij]quinoline (8la)

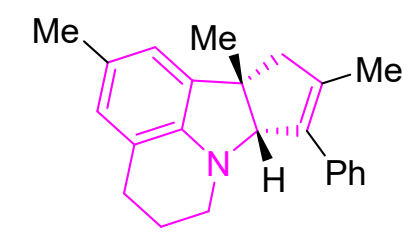

Chemical Formula: $\mathrm{C}_{23} \mathrm{H}_{25} \mathrm{~N}$

Exact Mass: 315.1987

8la was prepared according to general procedure 2.3 using $1 \mathrm{I}$ and $7 \mathrm{a}$ and was purified by silica gel column chromatography (petroleum ether/toluene 2:1-1:99) to obtain 8la as white oil (43\% yield). ${ }^{1} \mathrm{H}$ NMR (400 MHz, $\left.\mathrm{CDCl}_{3}\right) \delta$ 7.40-7.18 (m, 5H), $6.73(\mathrm{~s}, 1 \mathrm{H}), 6.61$ (s, 1H), $4.61(\mathrm{~s}, 1 \mathrm{H}), 2.90(\mathrm{~d}, J=16.7 \mathrm{~Hz}, 1 \mathrm{H}), 2.78-2.66(\mathrm{~m}, 1 \mathrm{H}), 2.60-2.40(\mathrm{~m}, 4 \mathrm{H}), 2.24$ (s, 3H), 1.89-1.73 (m, 2H), $1.72(\mathrm{~s}, 3 \mathrm{H}), 1.49(\mathrm{~s}, 3 \mathrm{H}) ;{ }^{13} \mathrm{C}$ NMR (100 MHz, CDCl 3$) \delta 145.4$, 138.2, 137.9, 135.5, 135.3, 128.2, 128.1, 127.0, 126.3, 126.1, 120.8, 118.0, 85.7, 53.6, 50.1, 45.7, 27.5, 24.0, 21.0, 15.2; HRMS: (ESI) calcd for $\mathrm{C}_{23} \mathrm{H}_{26} \mathrm{~N}^{+}[\mathrm{M}+\mathrm{H}]^{+} 316.2059$; found 316.2055 .

The enantiomeric purity was established by HPLC analysis using a chiral column: OJ-H column, $30{ }^{\circ} \mathrm{C}, n$-Hexane/i-Propanol $=95 / 5$ as eluent, $254 \mathrm{~nm}, 1 \mathrm{~mL} / \mathrm{min}$. $\mathrm{tR}=3 \mathrm{~min}$ (major), 4 min (minor).

Optical Rotation: $[\alpha]_{D}{ }^{26}-32.6$ (c 0.4 , ${ }^{\mathrm{P}} \mathrm{POH}$ ) for $94 \%$ ee. 


\section{LabSolutions 分析报告}

〈样品信息〉
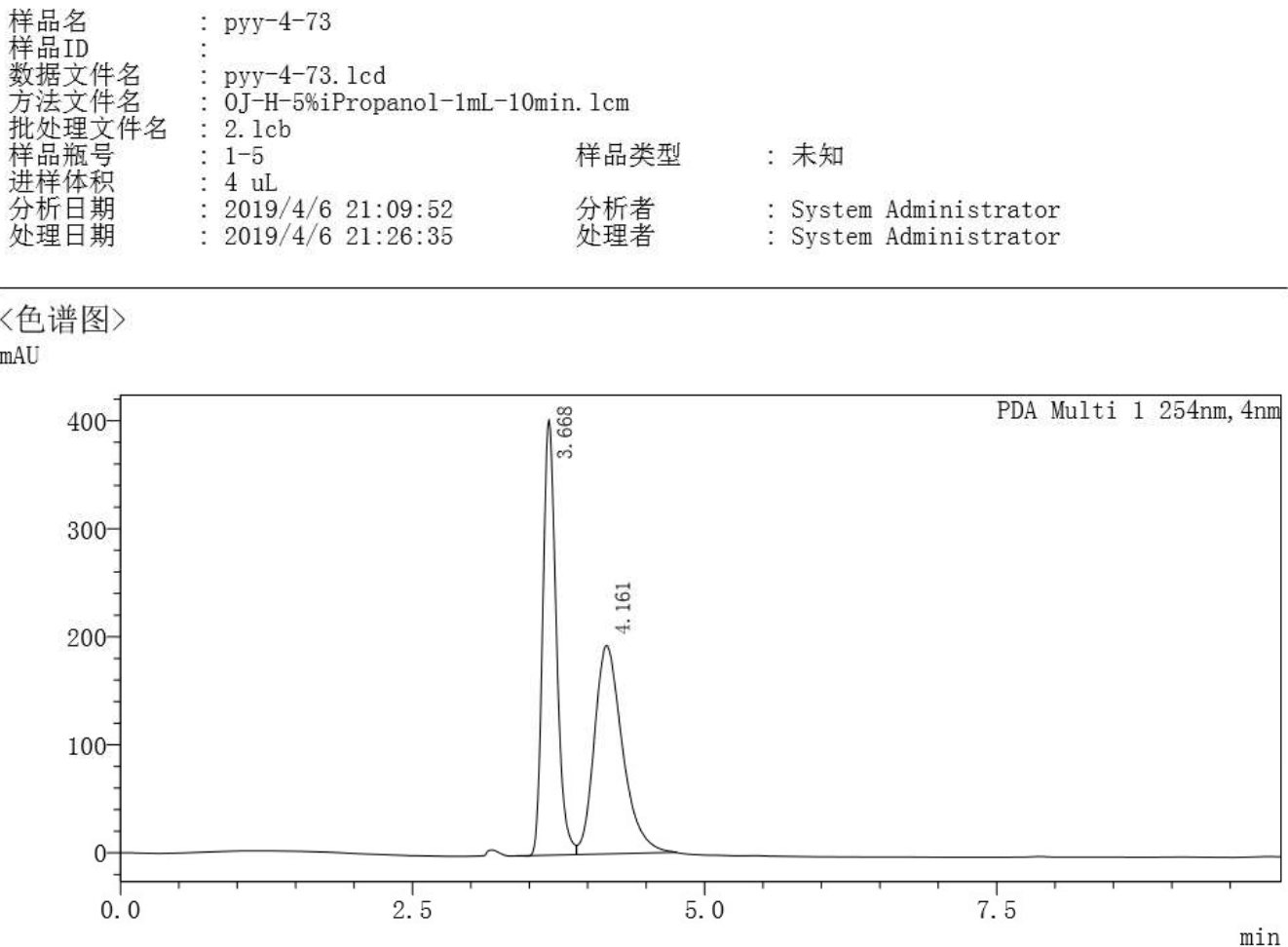

〈峰表〉

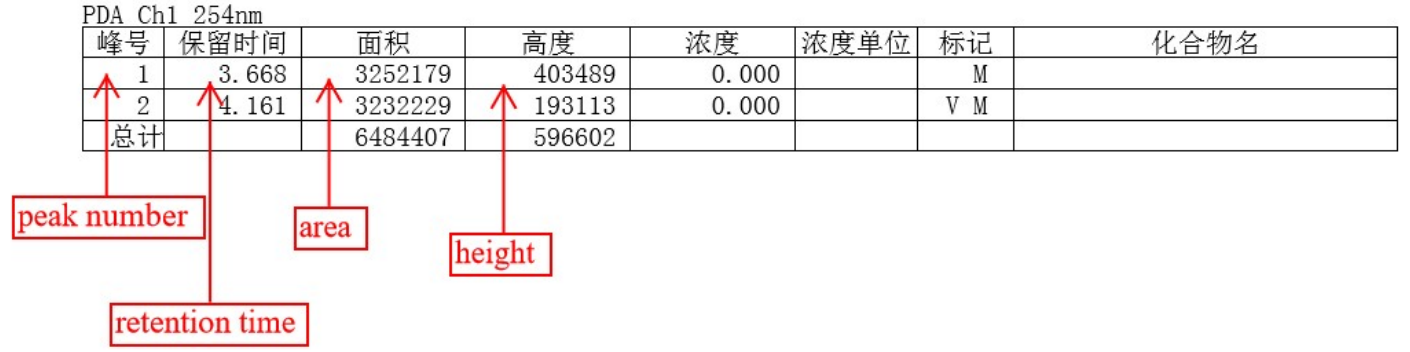




\section{An LabSolutions 分析报告}

\section{〈样品信息〉}

$\begin{array}{llll}\text { 样品名 } & \vdots \text { pyy-4-86 } & \\ \text { 样品ID } & \vdots & \\ \text { 数据文件名 } & \vdots \text { pyy-4-86. lcd } & \\ \text { 方法文件多 } & \vdots \text { OJ-H-5\% Propanol-1mL-10min. } 1 \mathrm{~cm} & \\ \text { 批处理文件名 } & \vdots 2.1 \mathrm{cb} & \\ \text { 样品瓶号 } & \vdots 1-6 & \text { 样品类型 } & \text { : 未知 } \\ \text { 进样体积 } & \vdots 4 \mathrm{uL} & & \\ \text { 分析日期 } & \vdots 2019 / 4 / 621: 20: 20 & \text { 分析者 } & \text { : System Administrator } \\ \text { 处理日期 } & : 2019 / 4 / 621: 25: 53 & \text { 处理者 } & \text { : System Administrator }\end{array}$

\section{〈色谱图〉}

$\mathrm{mAU}$

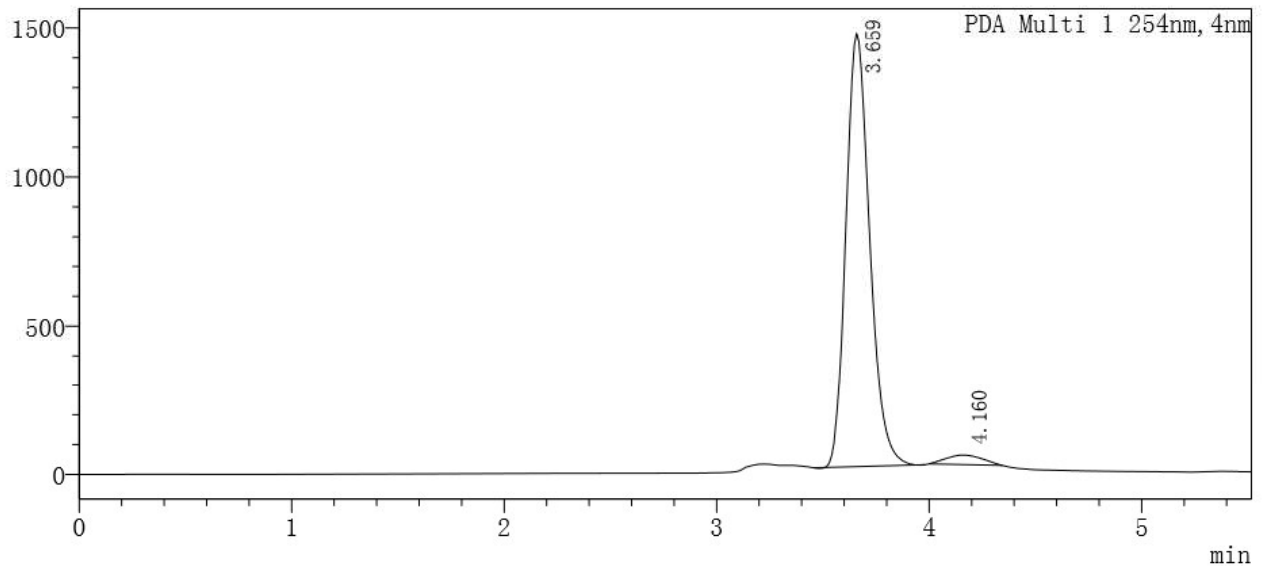

〈峰表〉

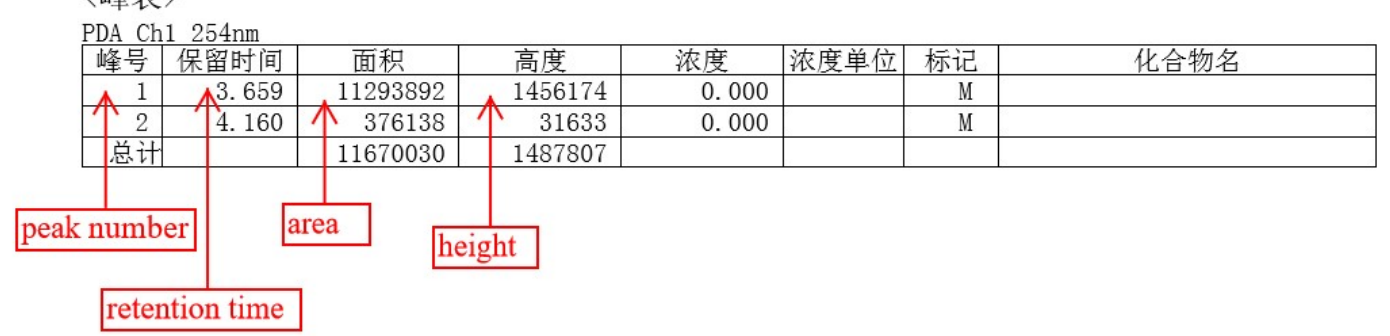


(3aR,8bS)-4-benzyl-2,8b-dimethyl-3-phenyl-1,3a,4,8b-tetrahydrocyclopenta[ $b]$ indole (8ma)

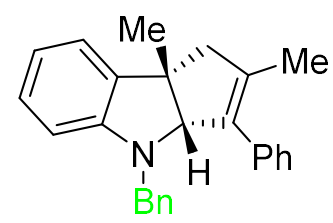

Chemical Formula: $\mathrm{C}_{26} \mathrm{H}_{25} \mathrm{~N}$

Exact Mass: 351.1987

$8 \mathrm{ma}$ was prepared according to general procedure 2.3 using $1 \mathrm{~m}$ and $7 \mathrm{a}$ and was purified by silica gel column chromatography (petroleum ether/toluene 2:1-1:99) to obtain $8 \mathrm{ma}$ as white oil (62\% yield). ${ }^{1} \mathrm{H} \mathrm{NMR}\left(400 \mathrm{MHz}, \mathrm{CDCl}_{3}\right) \delta 7.24-6.80(\mathrm{~m}, 12 \mathrm{H}), 6.63(\mathrm{t}, J=7.4 \mathrm{~Hz}$, $1 \mathrm{H}), 6.26(\mathrm{~d}, J=7.9 \mathrm{~Hz}, 1 \mathrm{H}), 4.68(\mathrm{~s}, 1 \mathrm{H}), 4.27(\mathrm{~d}, J=16.3 \mathrm{~Hz}, 1 \mathrm{H}), 3.81(\mathrm{~d}, J=16.3 \mathrm{~Hz}$, $1 \mathrm{H}), 2.91(\mathrm{~d}, J=16.1 \mathrm{~Hz}, 1 \mathrm{H}), 2.61(\mathrm{~d}, J=16.7 \mathrm{~Hz}, 1 \mathrm{H}), 1.65(\mathrm{~s}, 3 \mathrm{H}), 1.45(\mathrm{~s}, 3 \mathrm{H}) ;{ }^{13} \mathrm{C}$ NMR $\left(101 \mathrm{MHz}, \mathrm{CDCl}_{3}\right) \delta 150.1,138.9,137.7,137.6,136.2,129.0,128.6,128.2,128.1$, 127.8, 127.1, 126.6, 126.5, 125.3, 122.6, 116.5, 106.1, 83.3, 53.9, 50.4, 49.4, 28.1, 14.9; HRMS: (ESI) calcd for $\mathrm{C}_{26} \mathrm{H}_{26} \mathrm{~N}^{+}[\mathrm{M}+\mathrm{H}]^{+} 352.2059$; found 352.2047 .

The enantiomeric purity was established by HPLC analysis using a chiral column: OD-H column, $30{ }^{\circ} \mathrm{C}, n$-Hexane/i-Propanol $=98 / 2$ as eluent, $254 \mathrm{~nm}, 1 \mathrm{~mL} / \mathrm{min}$. $\mathrm{tR}=3 \mathrm{~min}$ (minor), 4 min (major).

Optical Rotation: $[\alpha]_{D^{27}}-94.4$ (c 0.4 , $\mathrm{PrOH}$ ) for $90 \%$ ee. 


\section{LabSolutions 分析报告}

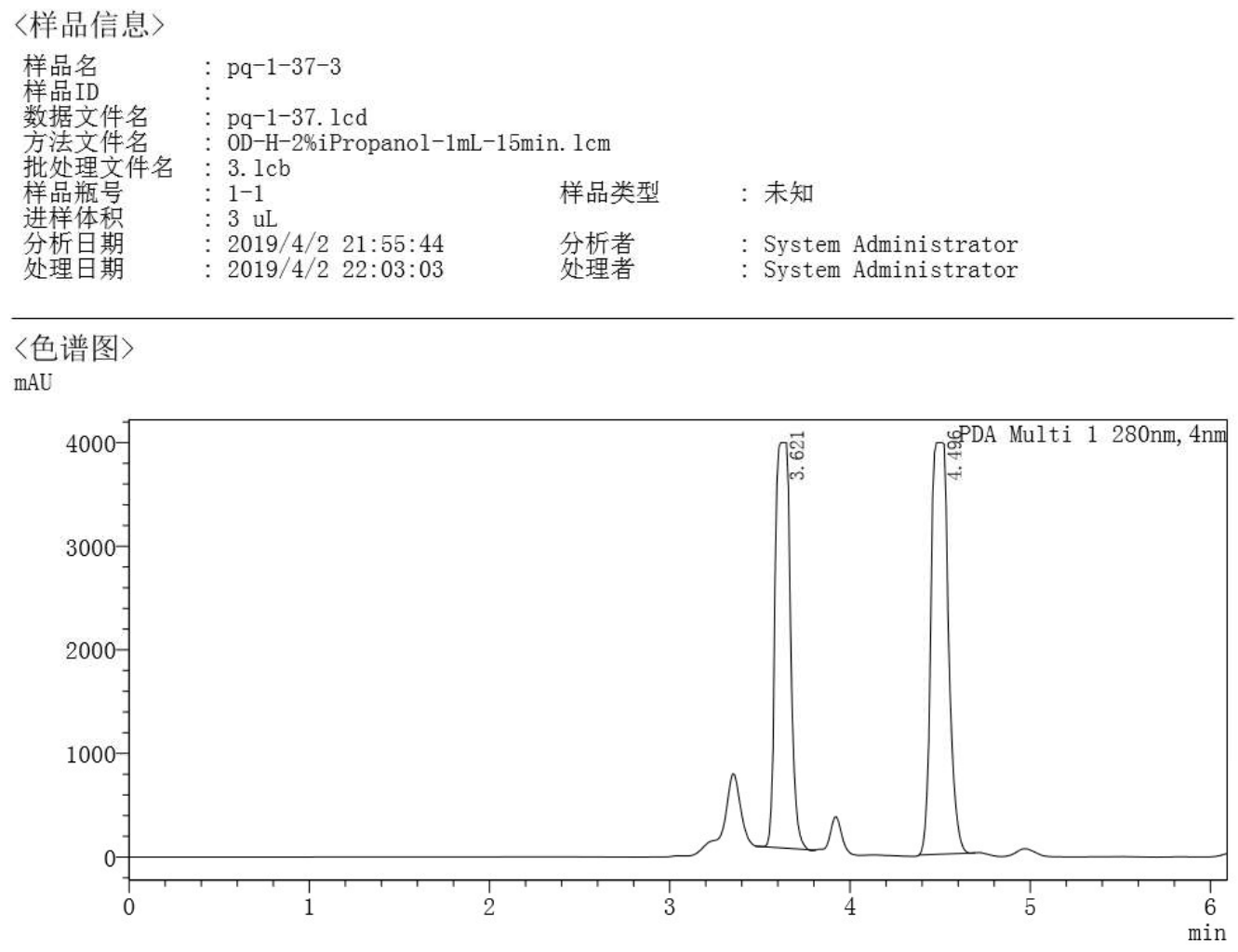

〈峰表〉

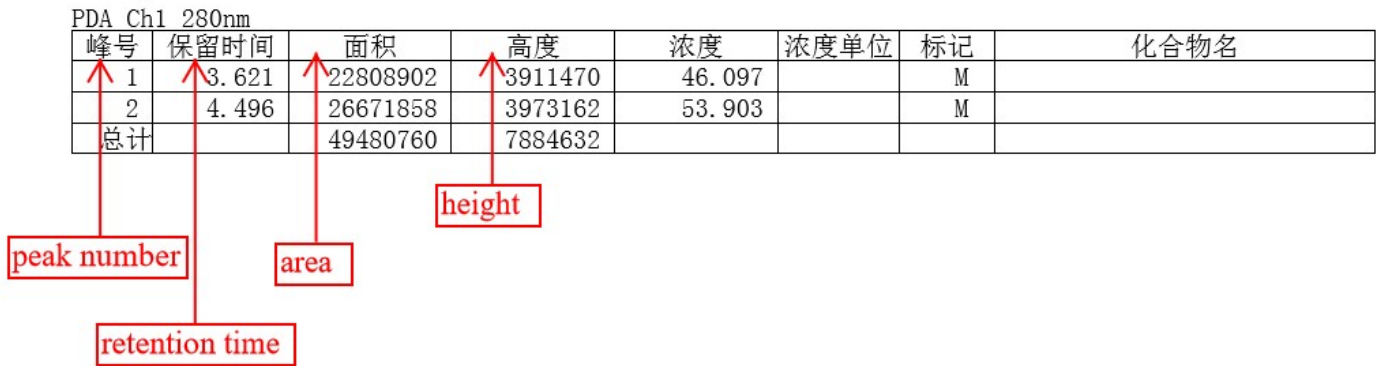




\section{LabSolutions 分析报告}

〈样品信息〉

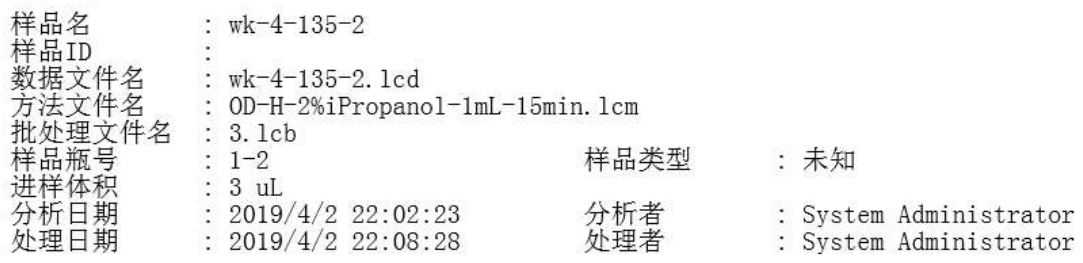

〈色谱图〉

mAU

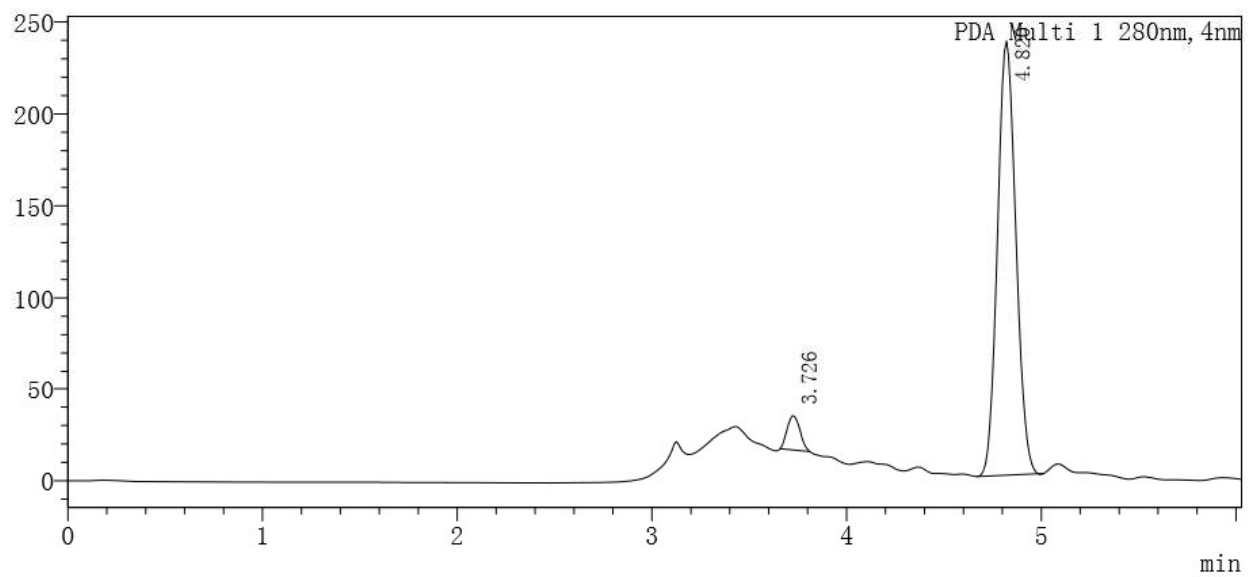

〈峰表〉

\begin{tabular}{|c|c|c|c|c|c|c|c|}
\hline 峰号 & 保留时间 & 面积 & 高度 & 浓度 & 浓度单位 & 标记 & 化合物名 \\
\hline 1 & 3.726 & 84365 & 18714 & 0.000 & & M & \\
\hline 本2 & 4.820 & 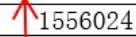 & 舟 236754 & 0.000 & & M & \\
\hline 总计 & & 1640388 & 255468 & & & & \\
\hline aumb & & & height & & & & \\
\hline
\end{tabular}

retention time 
(3aR,8bS)-8b-benzyl-2,4-dimethyl-3-phenyl-1,3a,4,8b-tetrahydrocyclopenta[ $b]$ indole (80a)

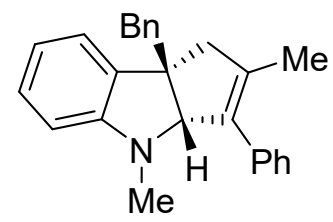

Chemical Formula: $\mathrm{C}_{26} \mathrm{H}_{25} \mathrm{~N}$

Exact Mass: 351.1987

$80 a$ was prepared according to general procedure 2.3 using 10 and $7 \mathrm{a}$ and was purified by silica gel column chromatography (petroleum ether/toluene 2:1-1:99) to obtain 8oa as white solid (50\% yield). ${ }^{1} \mathrm{H}$ NMR $\left(400 \mathrm{MHz}, \mathrm{CDCl}_{3}\right) \delta$ 7.34-7.27 (m, 2H), 7.23-7.16 (m, $6 \mathrm{H}), 7.08-7.02(\mathrm{~m}, 1 \mathrm{H}), 7.00-6.97(\mathrm{~m}, 3 \mathrm{H}), 6.68-6.62(\mathrm{~m}, 1 \mathrm{H}), 6.21(\mathrm{~d}, J=7.8 \mathrm{~Hz}, 1 \mathrm{H})$, $4.67(\mathrm{~s}, 1 \mathrm{H}), 3.18(\mathrm{~d}, J=13.3 \mathrm{~Hz}, 1 \mathrm{H}), 2.96(\mathrm{~d}, J=13.3 \mathrm{~Hz}, 1 \mathrm{H}), 2.86(\mathrm{~s}, 2 \mathrm{H}), 2.25$ (s, $3 \mathrm{H}), 1.61$ (s, 3H); ${ }^{13} \mathrm{C}$ NMR (100 MHz, $\left.\mathrm{CDCl}_{3}\right) \delta 151.5,138.4,137.8,136.9,136.0,135.9$, $130.1,128.5,128.1,127.7,126.4,126.2,123.5,116.5,106.7,82.8,54.6,51.6,46.2,35.2$, 14.9. HRMS: (ESI) calcd for $\mathrm{C}_{26} \mathrm{H}_{26} \mathrm{~N}^{+}[\mathrm{M}+\mathrm{H}]^{+} 352.2059$; found 352.2050 .

The enantiomeric purity was established by HPLC analysis using a chiral column: OD-H column, $30^{\circ} \mathrm{C}, n$-Hexane $/ i$-Propanol $=95 / 5$ as eluent, $254 \mathrm{~nm}, 1 \mathrm{~mL} / \mathrm{min}$. tR $=4.0 \mathrm{~min}$ (major), 4.2 min (minor).

Optical Rotation: $[\alpha]_{D^{27}}-23.3$ (c 0.4 , $\mathrm{PrOH}$ ) for $92 \%$ ee. 


\section{-nin Labsolutions 分析报告}

〈样品信息〉

$\begin{array}{llll}\text { 样品名 } & \vdots \text { pyy-4-72-2 } & \\ \text { 样品ID } & \vdots & \\ \text { 数据文件名 } & \vdots \text { pyy-4-72-2. lcd } & \\ \text { 方法文件名 } & \vdots \text { OD-H-5\% Propanol-1mL-15min. } 1 \mathrm{~cm} & \\ \text { 批处理文件名 } & \vdots 1.1 \mathrm{cb} & & \\ \text { 样品瓶号 } & \vdots 1-1 & \text { 样品类型 } & \text { : 未知 } \\ \text { 进样体积 } & \vdots 3 \mathrm{uL} & & \\ \text { 分析日期 } & \vdots 2019 / 3 / 2915: 37: 46 & \text { 分析者 } & \text { : System Administrator } \\ \text { 处理日期 } & \vdots 2019 / 3 / 2915: 52: 48 & \text { 处理者 } & \text { : System Administrator }\end{array}$

〈色谱图〉

$\mathrm{mAU}$

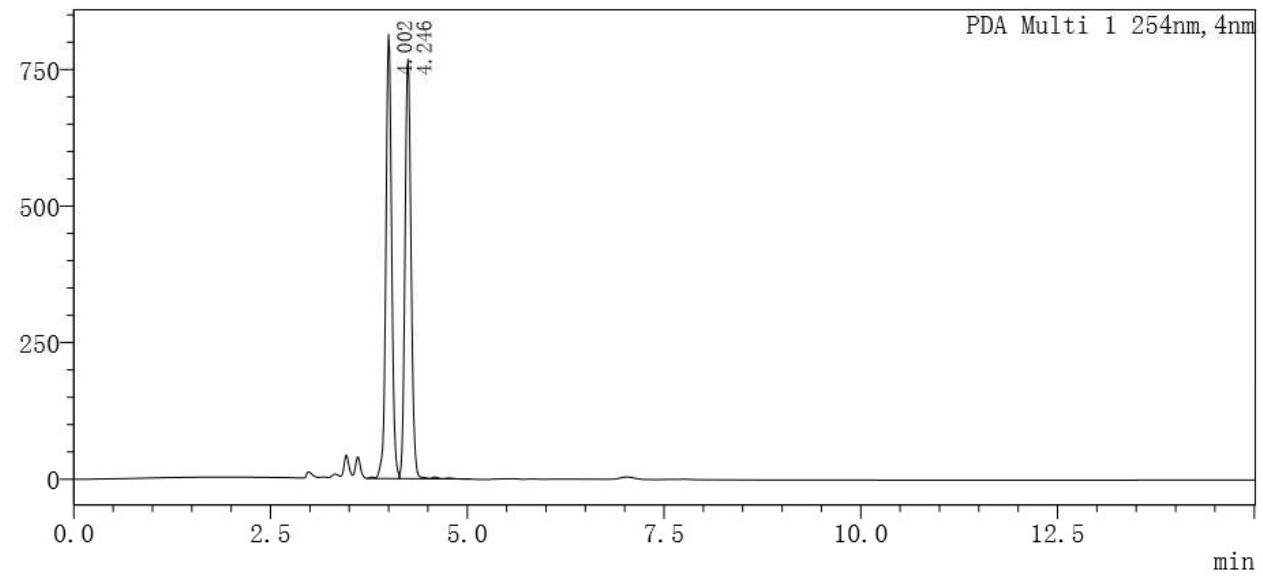

〈峰表〉

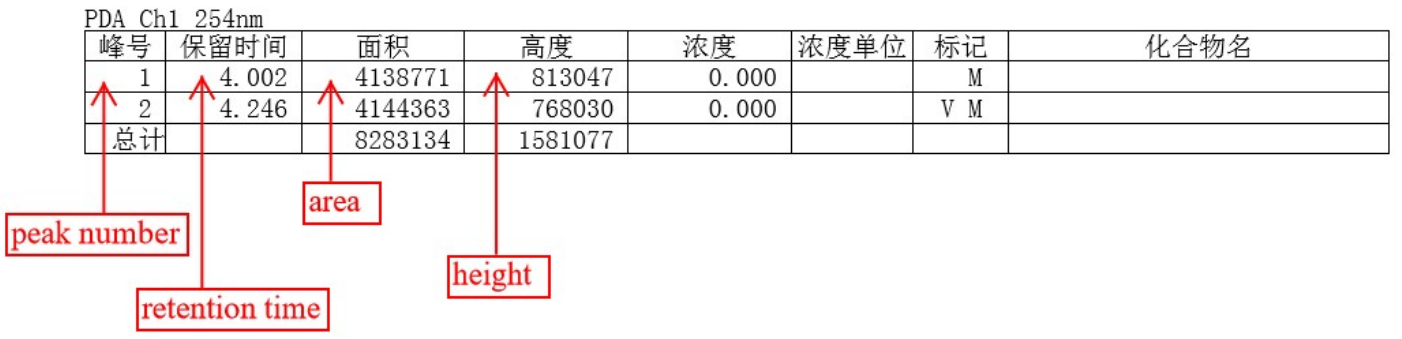




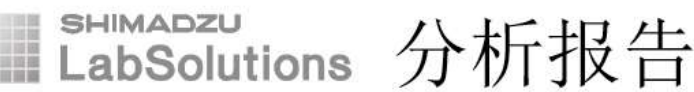
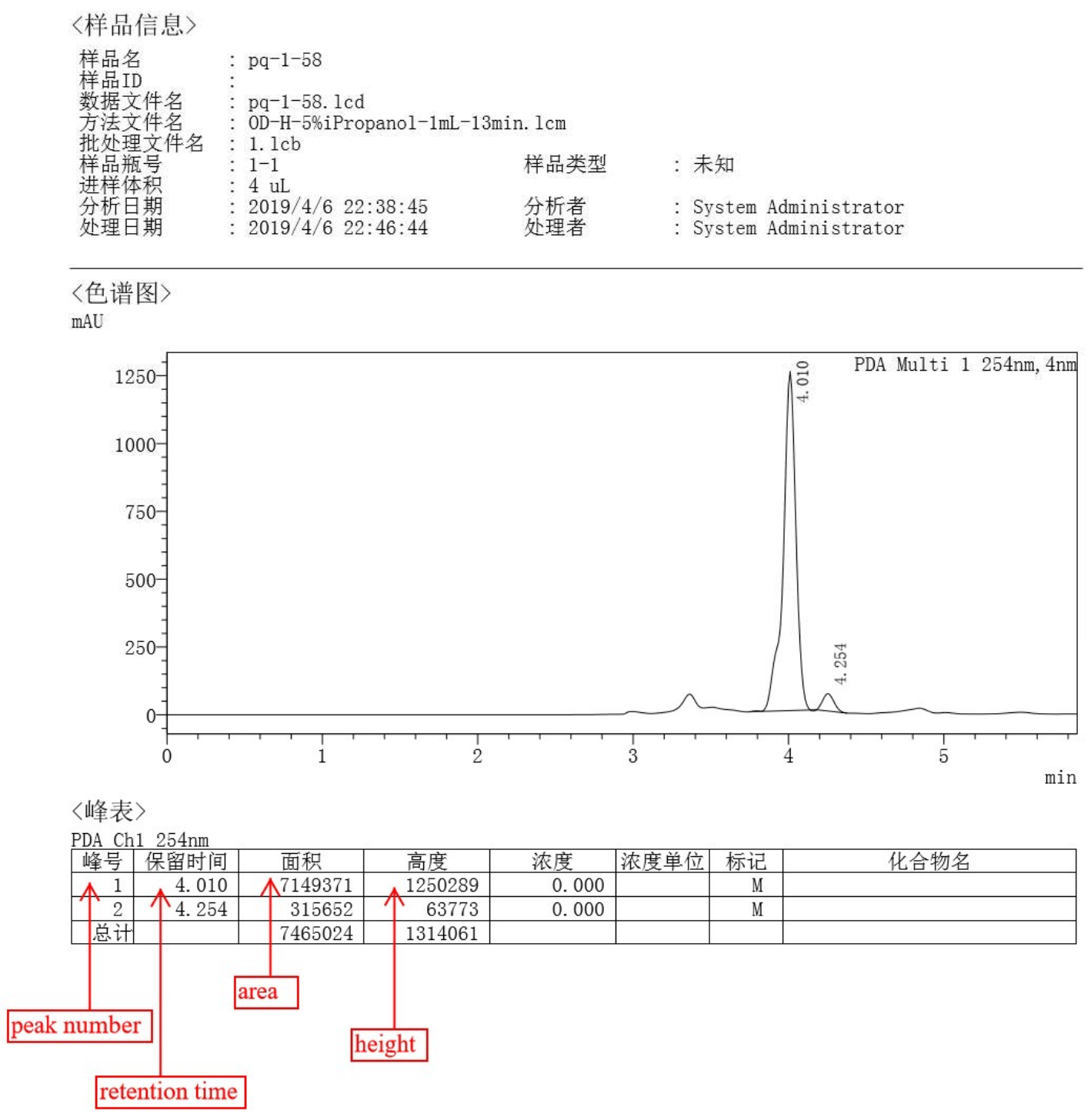
(3aR,8bS)-8b-hexyl-2,4-dimethyl-3-phenyl-1,3a,4,8b-tetrahydrocyclopenta[b]indole (8qa)

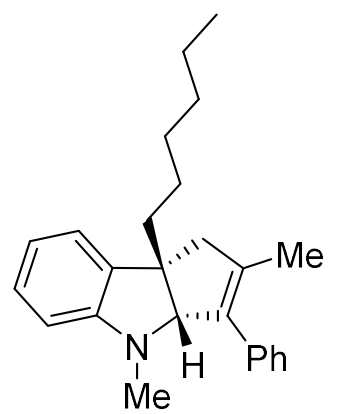

Chemical Formula: $\mathrm{C}_{25} \mathrm{H}_{31} \mathrm{~N}$

Exact Mass: 345.2457

8qa was prepared according to general procedure 2.3 using $1 \mathbf{q}$ and $7 \mathbf{a}$ and was purified by silica gel column chromatography (petroleum ether/toluene 2:1-1:99) to obtain $\mathbf{8 q a}$ as white solid (50\% yield). ${ }^{1} \mathrm{H}$ NMR $\left(400 \mathrm{MHz}, \mathrm{CDCl}_{3}\right) \delta$ 7.37-7.31 (m, $\left.2 \mathrm{H}\right), 7.30-7.21(\mathrm{~m}$, $3 \mathrm{H}), 7.09-6.99(\mathrm{~m}, 2 \mathrm{H}), 6.66-6.58(\mathrm{~m}, 1 \mathrm{H}), 6.28(\mathrm{~d}, J=7.7 \mathrm{~Hz}, 1 \mathrm{H}), 4.61(\mathrm{~s}, 1 \mathrm{H}), 2.82(\mathrm{~d}$, $J=16.5 \mathrm{~Hz}, 1 \mathrm{H}), 2.64(\mathrm{~d}, J=16.8 \mathrm{~Hz}, 1 \mathrm{H}), 2.49(\mathrm{~s}, 3 \mathrm{H}), 1.84-1.69(\mathrm{~m}, 2 \mathrm{H}), 1.66(\mathrm{~s}, 3 \mathrm{H})$, 1.35-1.22 (m, 7H), 1.20-1.07 (m, 1H), $0.87(\mathrm{t}, \mathrm{J}=8.4 \mathrm{~Hz}, 3 \mathrm{H}) ;{ }^{13} \mathrm{C} \mathrm{NMR}\left(100 \mathrm{MHz}, \mathrm{CDCl}_{3}\right)$ ठ 151.3, 138.0, 137.6, 136.5, 136.0, 128.5, 128.1, 127.7, 126.4, 122.9, 116.4, 106.1, 83.8, 53.3, 52.5, 40.9, 35.0, 31.8, 29.9, 24.7, 22.7, 14.9, 14.1; HRMS: (ESI) calcd for $\mathrm{C}_{25} \mathrm{H}_{32} \mathrm{~N}^{+}[\mathrm{M}+\mathrm{H}]^{+} 346.2529$; found 346.2519 .

The enantiomeric purity was established by HPLC analysis using a chiral column: OJ-H column, $30{ }^{\circ} \mathrm{C}, n$-Hexane $/$-Propanol $=95 / 5$ as eluent, $254 \mathrm{~nm}, 1 \mathrm{~mL} / \mathrm{min}$. tR $=3.4 \mathrm{~min}$ (major), 4.5 min (minor).

Optical Rotation: $[\alpha]_{D^{27}}-119.9$ (c 0.4, $\left.{ }^{i} \mathrm{PrOH}\right)$ for $94 \%$ ee. 


\section{Lill SHMADZu}

〈样品信息〉

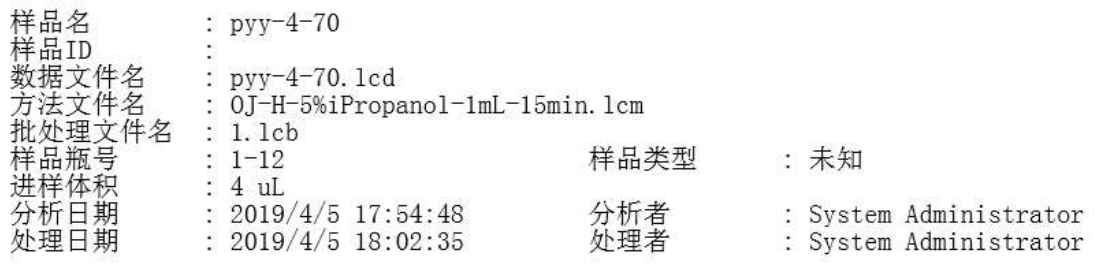

〈色谱图〉

$\mathrm{mAU}$

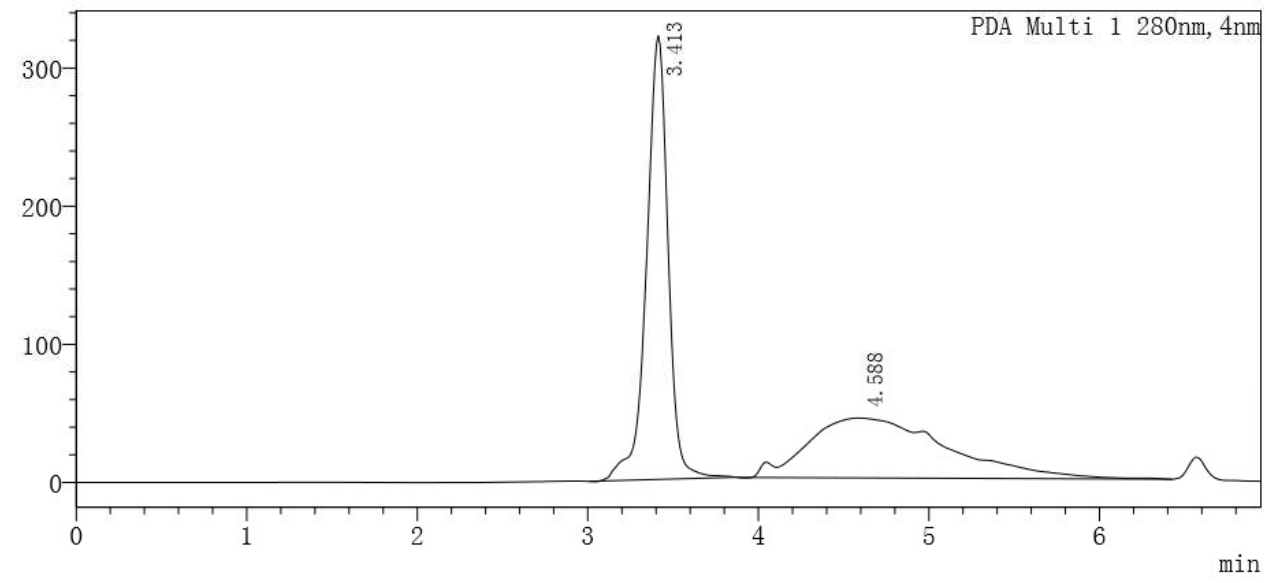

〈峰表〉

\begin{tabular}{|c|c|c|c|c|c|c|c|}
\hline 峰号 & 保留时间 & 面积 & 高度 & 浓度 & 浓度单位 & 标记 & 化合物名 \\
\hline$\triangle 1$ & 3.413 & A2829099 & 321447 & 0.000 & & M & \\
\hline 2 & 4.588 & 2491277 & 木 43397 & 0.000 & & M & \\
\hline 总计 & & 5320377 & 364844 & & & & \\
\hline
\end{tabular}

retention time 


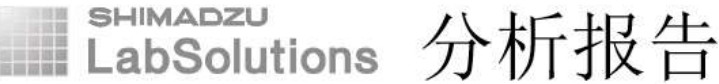

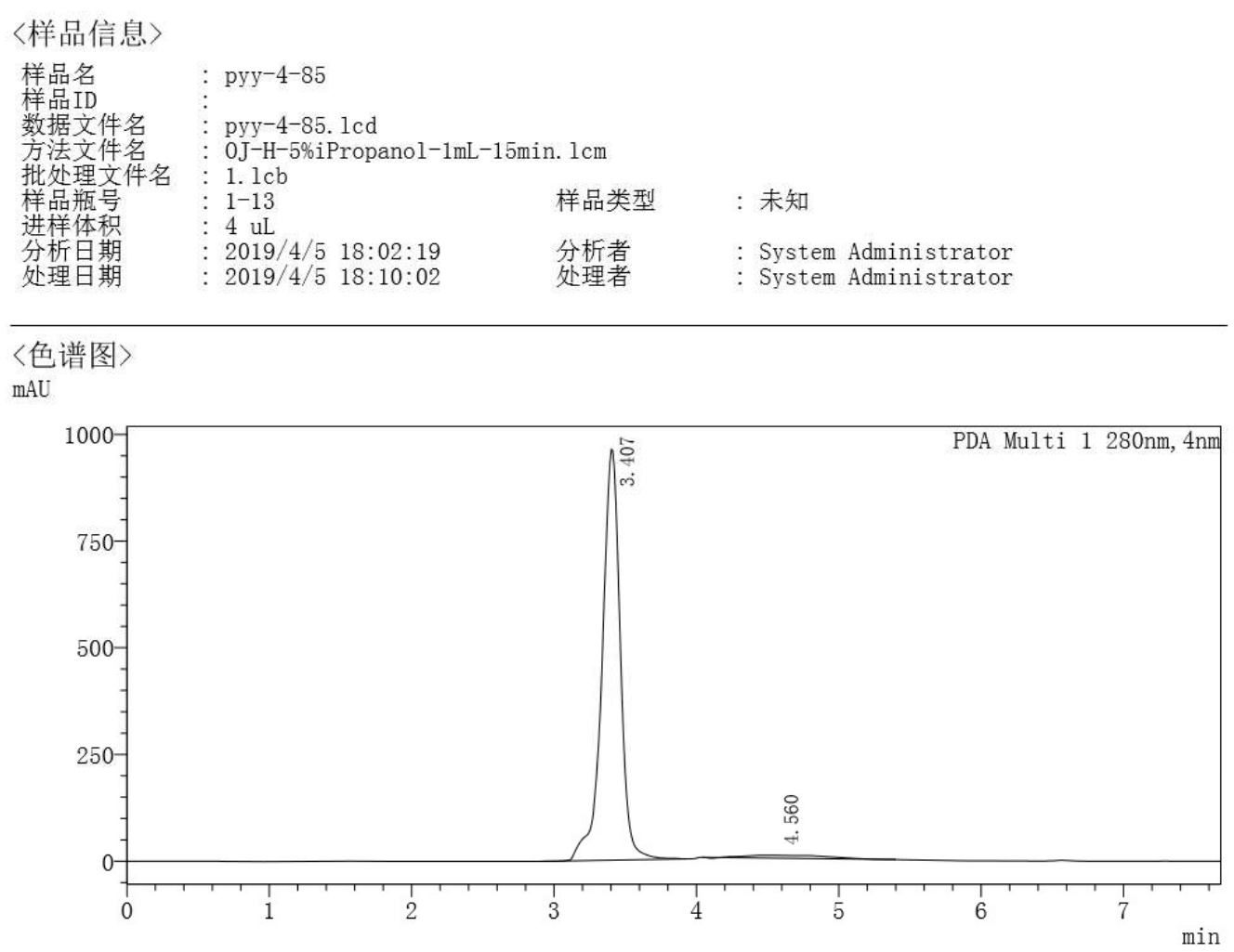

〈峰表〉

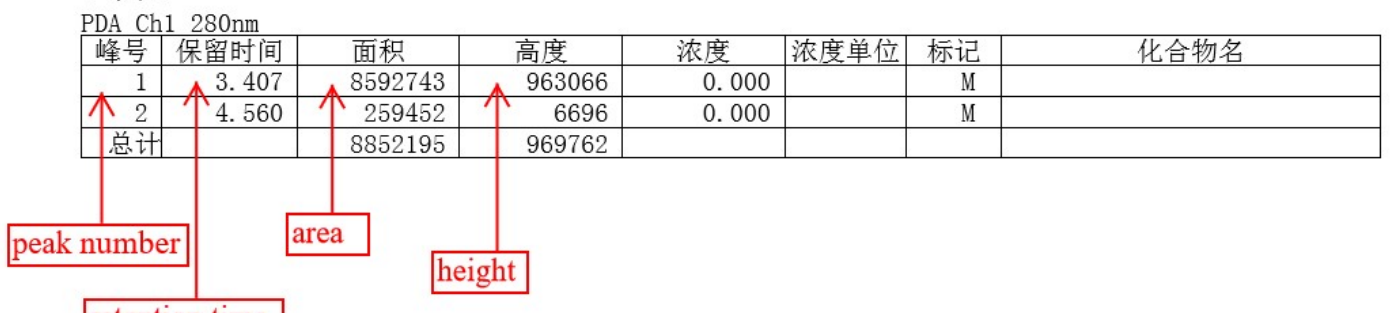

retention time 
(3aR,8bS)-4,8b-dimethyl-2-pentyl-3-phenyl-1,3a,4,8b-tetrahydrocyclopenta[b]indole (8ab)

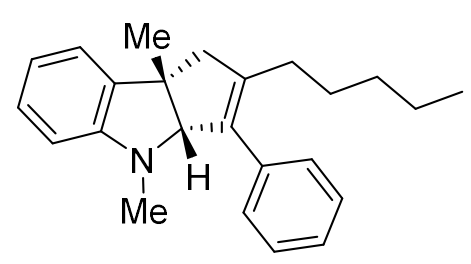

Chemical Formula: $\mathrm{C}_{24} \mathrm{H}_{29} \mathrm{~N}$

Exact Mass: 331.2300

8ab was prepared according to general procedure 2.3 using $1 \mathbf{a}$ and $7 \mathbf{b}$ and was purified by silica gel column chromatography (petroleum ether/toluene 2:1-1:99) to obtain $8 \mathrm{ab}$ as colorless oil (45\% yield). ${ }^{1} \mathrm{H}$ NMR $\left(400 \mathrm{MHz}, \mathrm{CDCl}_{3}\right) \delta$ 7.44-7.35 (m, 2H), 7.34-7.25 (m, $3 \mathrm{H})$, 7.15-7.07 (m, 2H), 6.74-6.65 (m, 1H), 6.39-6.32 (m, 1H), $4.48(\mathrm{~s}, 1 \mathrm{H}), 2.87$ (dd, $J=$ 16.6, $1.7 \mathrm{~Hz}, 1 \mathrm{H}), 2.65(\mathrm{~d}, J=16.6 \mathrm{~Hz}, 1 \mathrm{H}), 2.51(\mathrm{~s}, 3 \mathrm{H}), 2.20-2.01(\mathrm{~m}, 2 \mathrm{H}), 1.54(\mathrm{~s}, 3 \mathrm{H})$, 1.46-1.32 (m, 2H), 1.29-1.14 (m, 4H), $0.86(\mathrm{t}, \mathrm{J}=6.9 \mathrm{~Hz}, 3 \mathrm{H}) ;{ }^{13} \mathrm{C} \mathrm{NMR}\left(101 \mathrm{MHz}, \mathrm{CDCl}_{3}\right)$ $\delta 151.3,142.4,138.2,137.7,135.9,128.6,128.2,127.7,126.5,122.5,116.8,106.3,87.4$, 50.9, 49.5, 35.3, 31.7, 28.9, 27.9, 27.7, 22.5, 14.0; HRMS: (ESI) calcd for $\mathrm{C}_{24} \mathrm{H}_{30} \mathrm{~N}^{+}[\mathrm{M}+\mathrm{H}]^{+}$ 332.2373; found 332.2374.

The enantiomeric purity was established by HPLC analysis using a chiral column: OJ-H column, $30^{\circ} \mathrm{C}, n$-Hexane/i-Propanol $=99 / 1$ as eluent, $254 \mathrm{~nm}, 0.5 \mathrm{~mL} / \mathrm{min}$. $\mathrm{tR}=7 \mathrm{~min}$ (major), 9 min (minor).

Optical Rotation: $[\alpha]_{D^{27}}-18.0$ (c 0.4 , $\left.{ }^{i} \mathrm{PrOH}\right)$ for $91 \%$ ee. 


\section{An SHIMADZZ}

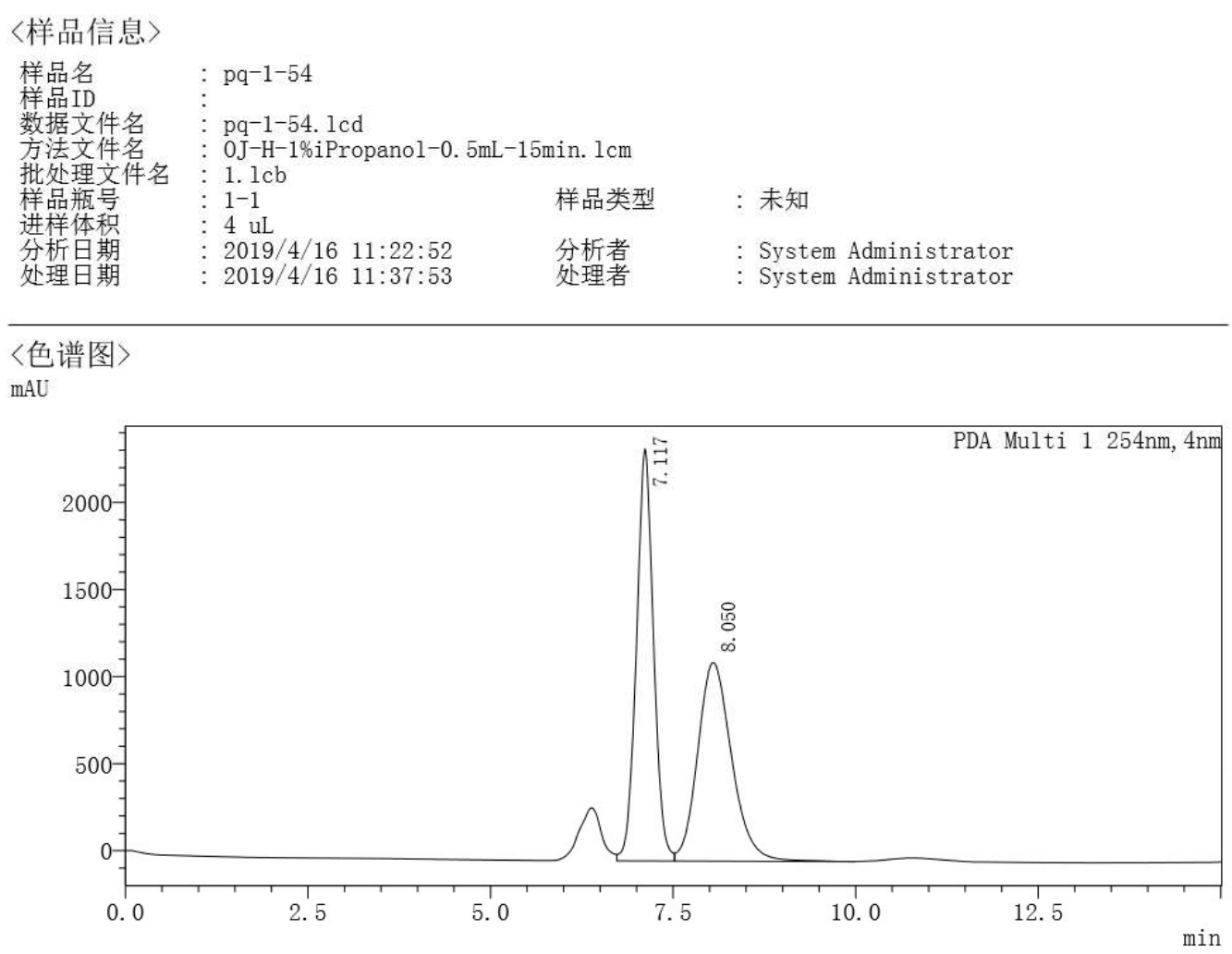

〈峰表〉

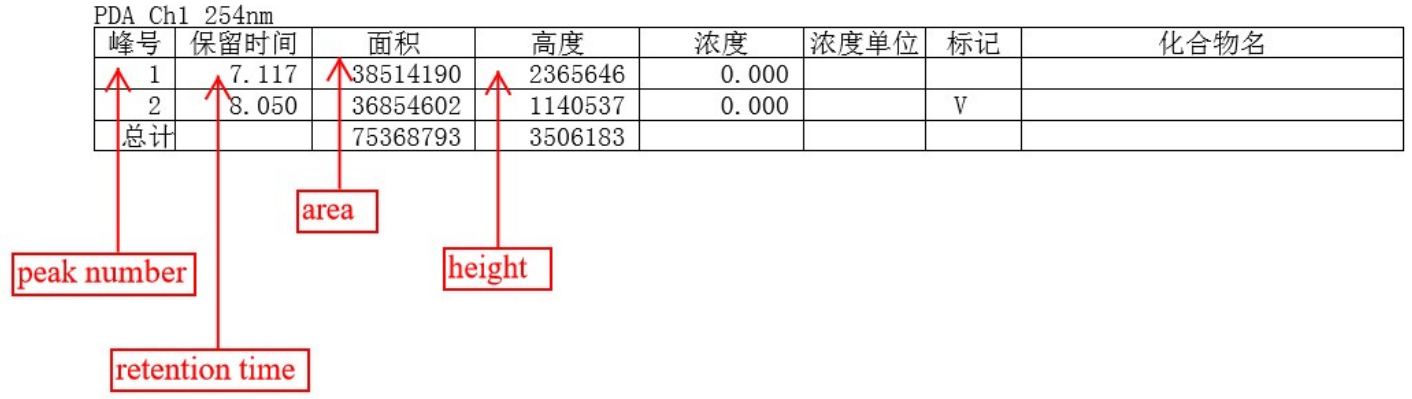




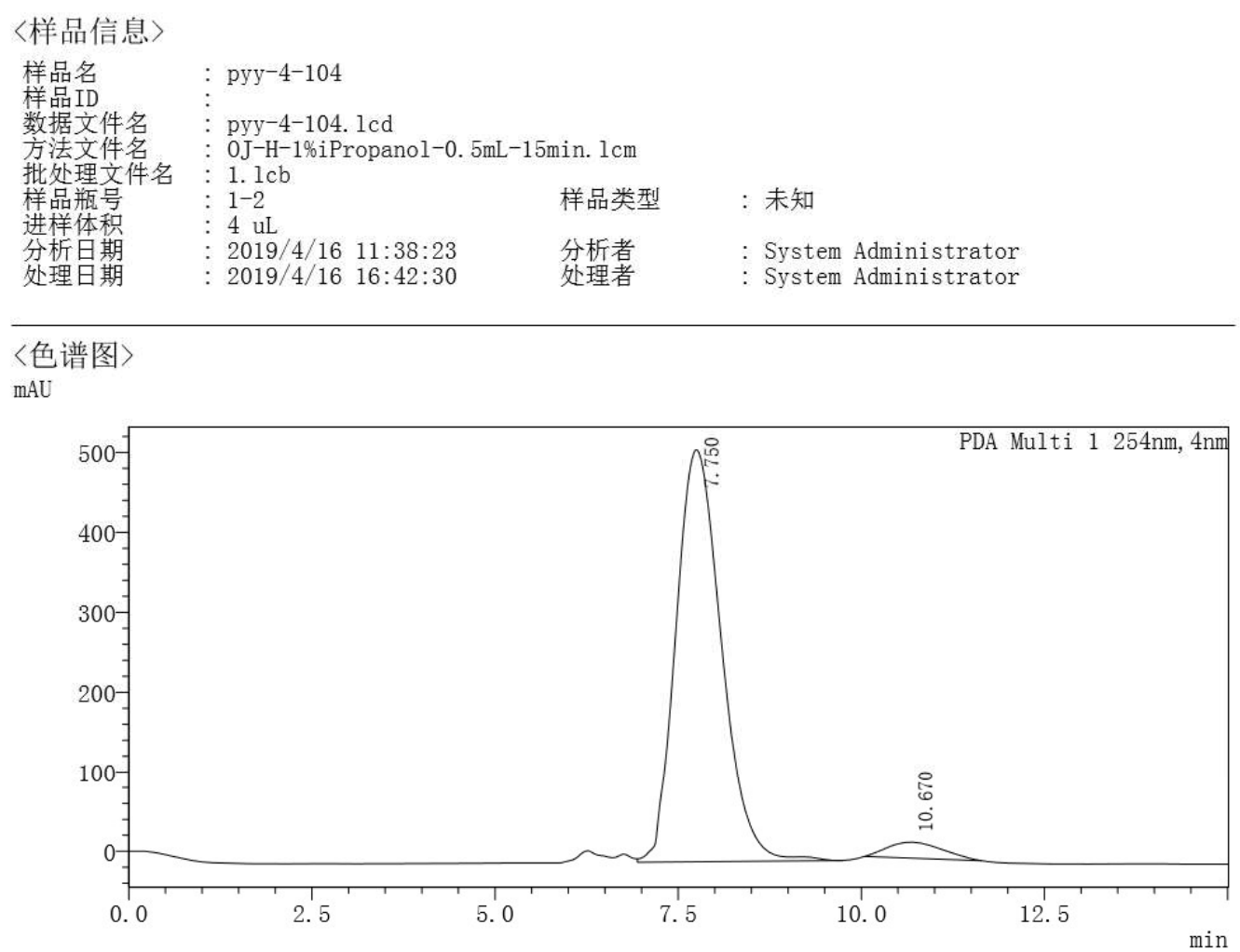

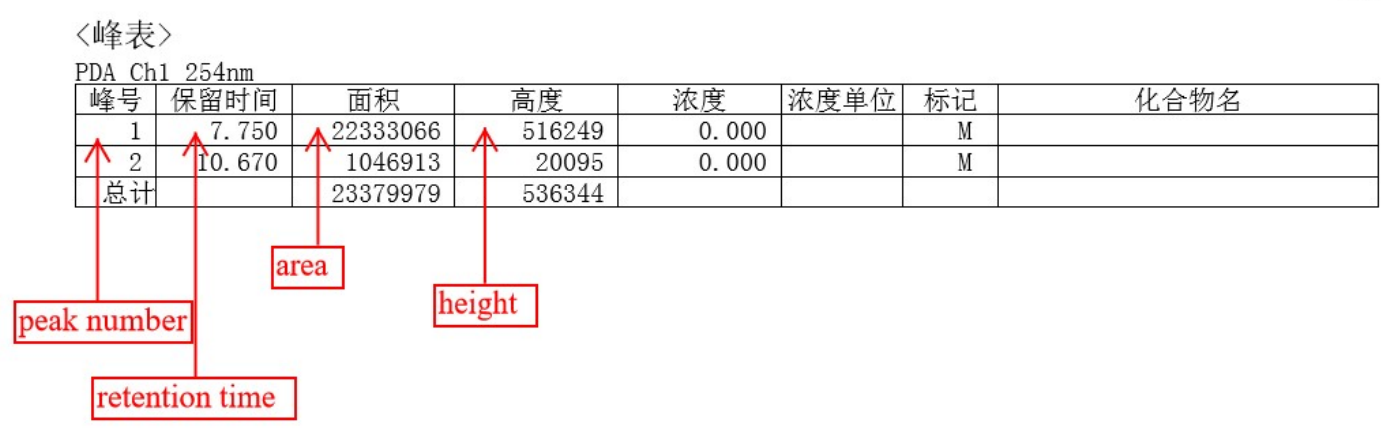


(3aR,8bS)-3-(4-methoxyphenyl)-2,4,8b-trimethyl-1,3a,4,8b-

tetrahydrocyclopenta[b]indole (8ac)

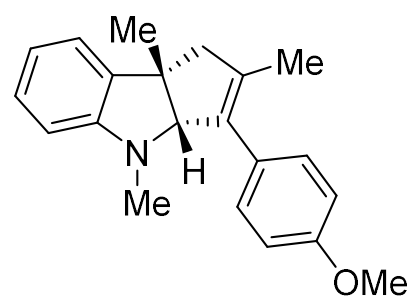

Chemical Formula: $\mathrm{C}_{21} \mathrm{H}_{23} \mathrm{NO}$

Exact Mass: 305.1780

8ac was prepared according to general procedure 2.3 using $1 \mathrm{a}$ and $7 \mathrm{c}$ and was purified by silica gel column chromatography (petroleum ether/toluene 2:1-1:99) to obtain 8ac as colorless oil (65\% yield). ${ }^{1} \mathrm{H}$ NMR $\left(400 \mathrm{MHz}, \mathrm{CDCl}_{3}\right) \delta$ 7.34-7.23 (m, 2H), 7.14-7.04 (m, 2H), 7.02-6.88 (m, 2H), 6.74-6.58 (m, 1H), $6.37(\mathrm{~d}, J=8.2 \mathrm{~Hz}, 1 \mathrm{H}), 4.49(\mathrm{~d}, J=1.3 \mathrm{~Hz}$, 1H), $3.88(\mathrm{~s}, 3 \mathrm{H}), 2.90(\mathrm{~d}, J=16.8 \mathrm{~Hz}, 1 \mathrm{H}), 2.61(\mathrm{~d}, J=16.8 \mathrm{~Hz}, 1 \mathrm{H}), 2.56(\mathrm{~s}, 3 \mathrm{H}), 1.74$ (s, 3H), 1.55 (s, 3H); ${ }^{13} \mathrm{C}$ NMR (101 MHz, $\left.\mathrm{CDCl}_{3}\right) \delta 158.2,151.2,137.7,137.2,135.4$, 130.3, 129.6, 127.8, 122.5, 116.8, 113.6, 106.3, 86.9, 55.2, 53.9, 49.4, 35.3, 28.0, 15.1; HRMS: (ESI) calcd for $\mathrm{C}_{21} \mathrm{H}_{24} \mathrm{ON}^{+}[\mathrm{M}+\mathrm{H}]^{+} 306.1852$; found 306.1848 .

The enantiomeric purity was established by HPLC analysis using a chiral column: IA column, $30{ }^{\circ} \mathrm{C}, n$-Hexane $/ \mathrm{i}$-Propanol $=97 / 3$ as eluent, $254 \mathrm{~nm}, 0.7 \mathrm{~mL} / \mathrm{min}$. $\mathrm{tR}=15 \mathrm{~min}$ (major), 16 min (minor).

Optical Rotation: $[\alpha]_{D^{27}}-55.7$ (c 0.4 , $\left.{ }^{\mathrm{P}} \mathrm{POH}\right)$ for $85 \%$ ee. 
〈样品信息〉

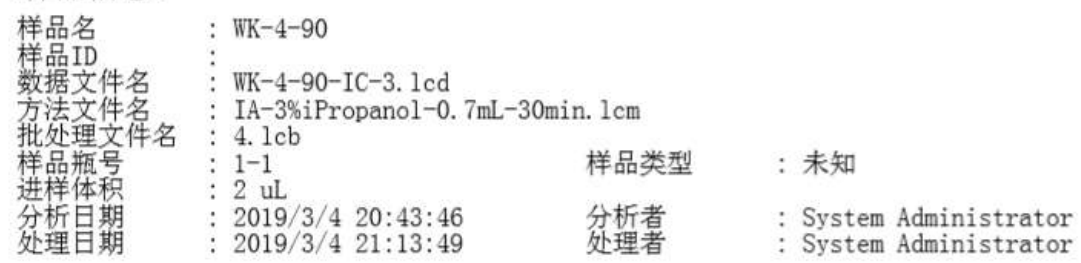

〈色谱图〉

$\mathrm{mAU}$

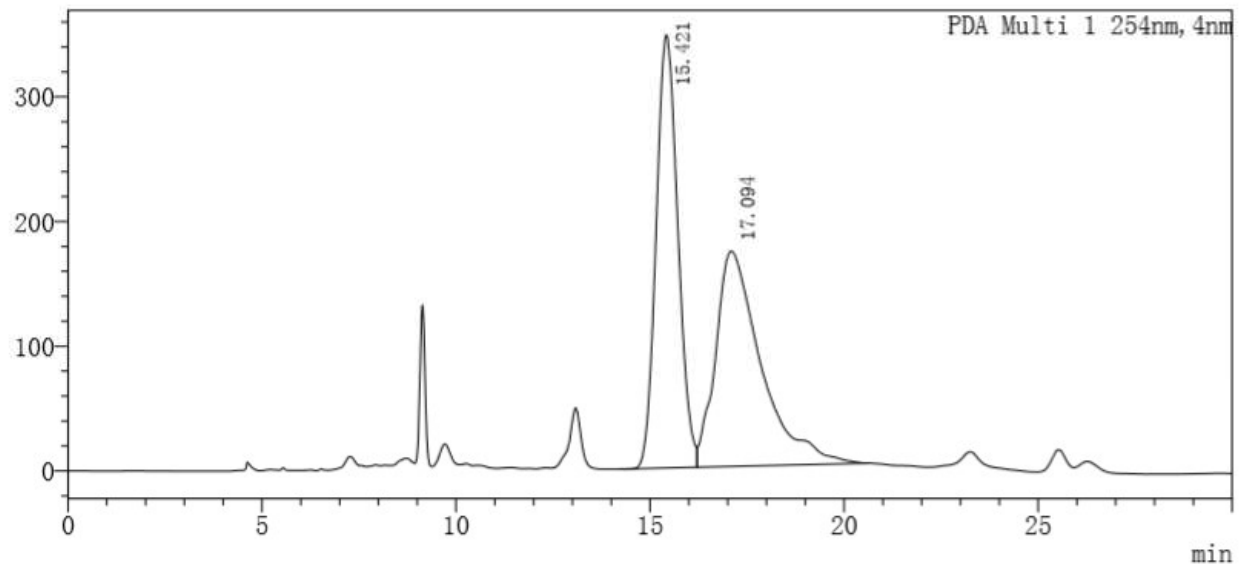

〈峰表〉

PDA Ch1 254nm

\begin{tabular}{|r|r|r|r|r|r|r|r|}
\hline 峰号 & 保留时间 & 面积 & 高度 & 浓度 & 浓度单位 & 标记 & 化合物名 \\
\hline 1 & 15.421 & 13515971 & 347323 & 0.000 & & M & \\
\hline 2 & 17.094 & 14027756 & 172541 & 0.000 & & V M & \\
\hline 总计 & & 27543727 & 519864 & & & & \\
\hline
\end{tabular}


〈样品信息〉

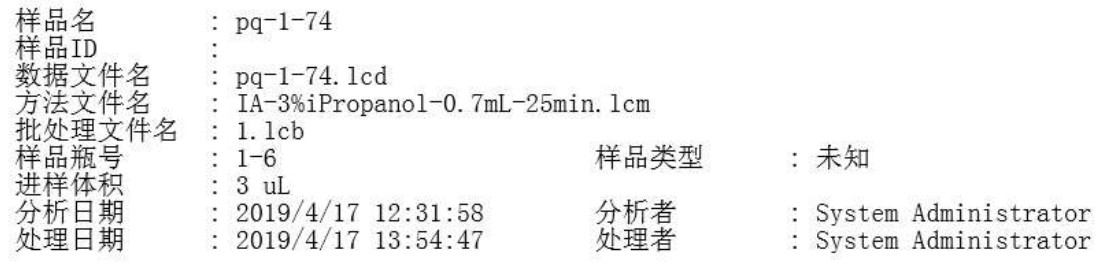

〈色谱图〉

$\mathrm{mAU}$

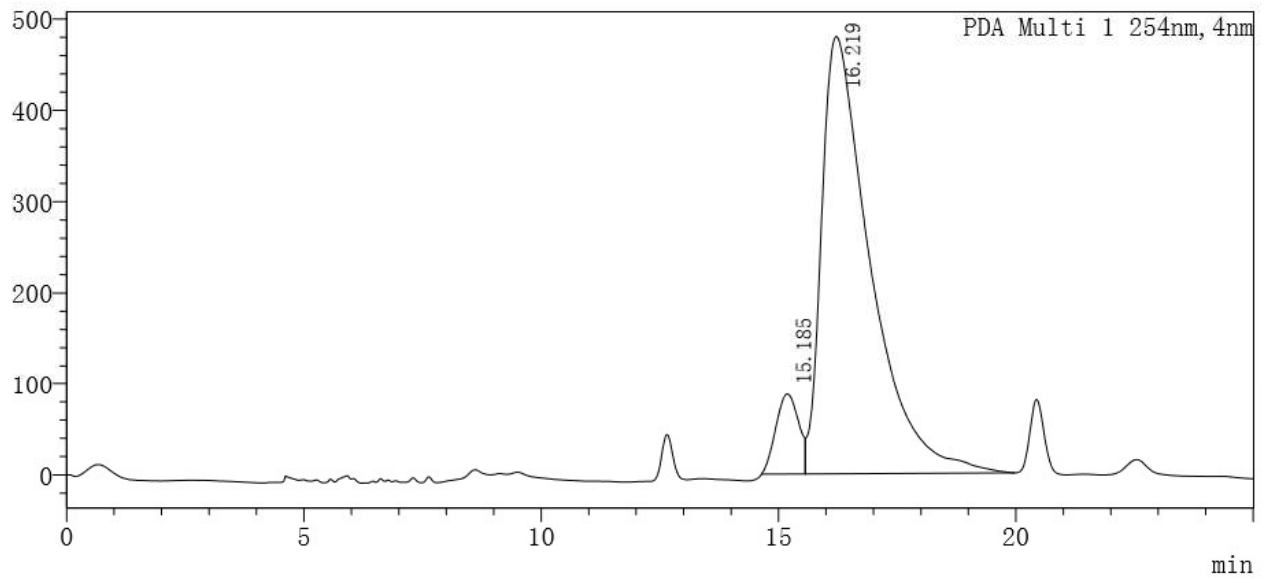

〈峰表〉

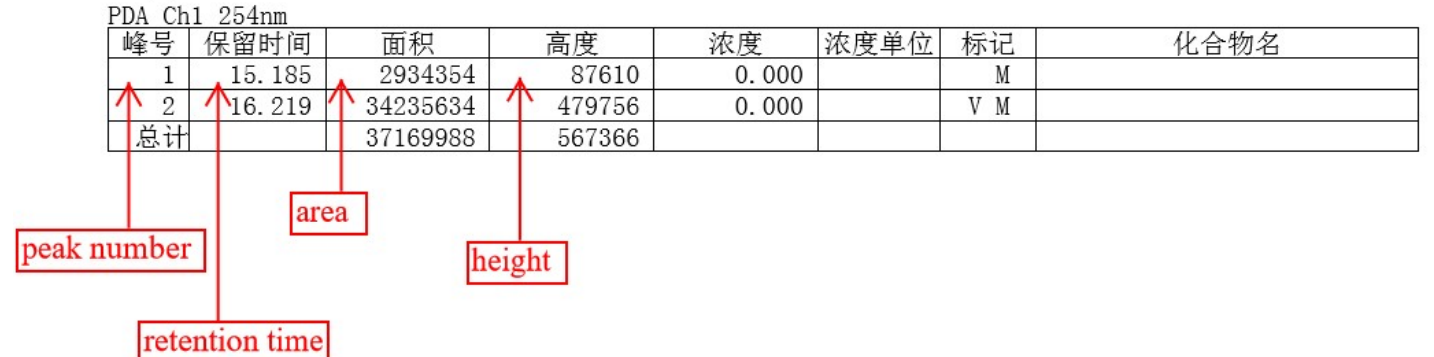

retention time 
(3aR,8bS)-2,4,8b-trimethyl-3-(p-tolyl)-1,3a,4,8b-tetrahydrocyclopenta[b]indole (8ad)

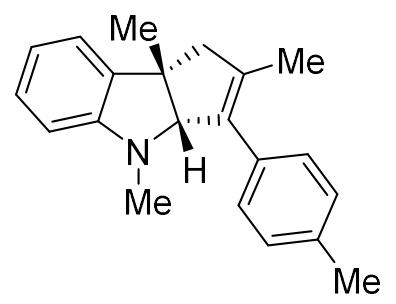

Chemical Formula: $\mathrm{C}_{21} \mathrm{H}_{23} \mathrm{~N}$

Exact Mass: 289.1830

$\mathbf{8 a d}$ was prepared according to general procedure 2.3 using $\mathbf{1 a}$ and $\mathbf{7 d}$ and was purified by silica gel column chromatography (petroleum ether/toluene 2:1-1:99) to obtain 8ad as colorless oil (45\% yield). ${ }^{1} \mathrm{H}$ NMR $\left(400 \mathrm{MHz}, \mathrm{CDCl}_{3}\right)$ ठ 7.21-7.16 (m, 4H), 7.08-7.05 (m, 2H), $6.65(\mathrm{t}, J=7.4 \mathrm{~Hz}, 1 \mathrm{H}), 6.32(\mathrm{~d}, J=8.0 \mathrm{~Hz}, 1 \mathrm{H}), 4.48(\mathrm{~s}, 1 \mathrm{H}), 2.87(\mathrm{~d}, J=16.7 \mathrm{~Hz}$, 1H), $2.57(\mathrm{~d}, J=16.8 \mathrm{~Hz}, 1 \mathrm{H}), 2.51(\mathrm{~s}, 3 \mathrm{H}), 2.38(\mathrm{~s}, 3 \mathrm{H}), 1.70(\mathrm{~s}, 3 \mathrm{H}), 1.51(\mathrm{~s}, 3 \mathrm{H}) ;{ }^{13} \mathrm{C}$ NMR $\left(100 \mathrm{MHz}, \mathrm{CDCl}_{3}\right) \delta 151.2,137.7,137.6,136.1,135.7,134.9,128.9,128.4,127.8$, 122.5, 116.8, 106.3, 86.9, 54.0, 49.4, 35.3, 28.0, 21.3, 15.1; HRMS: (ESI) calcd for $\mathrm{C}_{21} \mathrm{H}_{24} \mathrm{~N}+[\mathrm{M}+\mathrm{H}]^{+}$290.1903; found 290.1901.

The enantiomeric purity was established by HPLC analysis using a chiral column: OJ-H column, $30{ }^{\circ} \mathrm{C}, n$-Hexane $/ i$-Propanol $=95 / 5$ as eluent, $254 \mathrm{~nm}, 1 \mathrm{~mL} / \mathrm{min}$. tR $=4 \mathrm{~min}$ (major), 7 min (minor).

Optical Rotation: $[\alpha]_{D^{27}}-16.3$ (c 0.4 , $\mathrm{PrOH}$ ) for $91 \%$ ee. 


\section{SHIMADZU \\ 分析报告}

〈样品信息〉
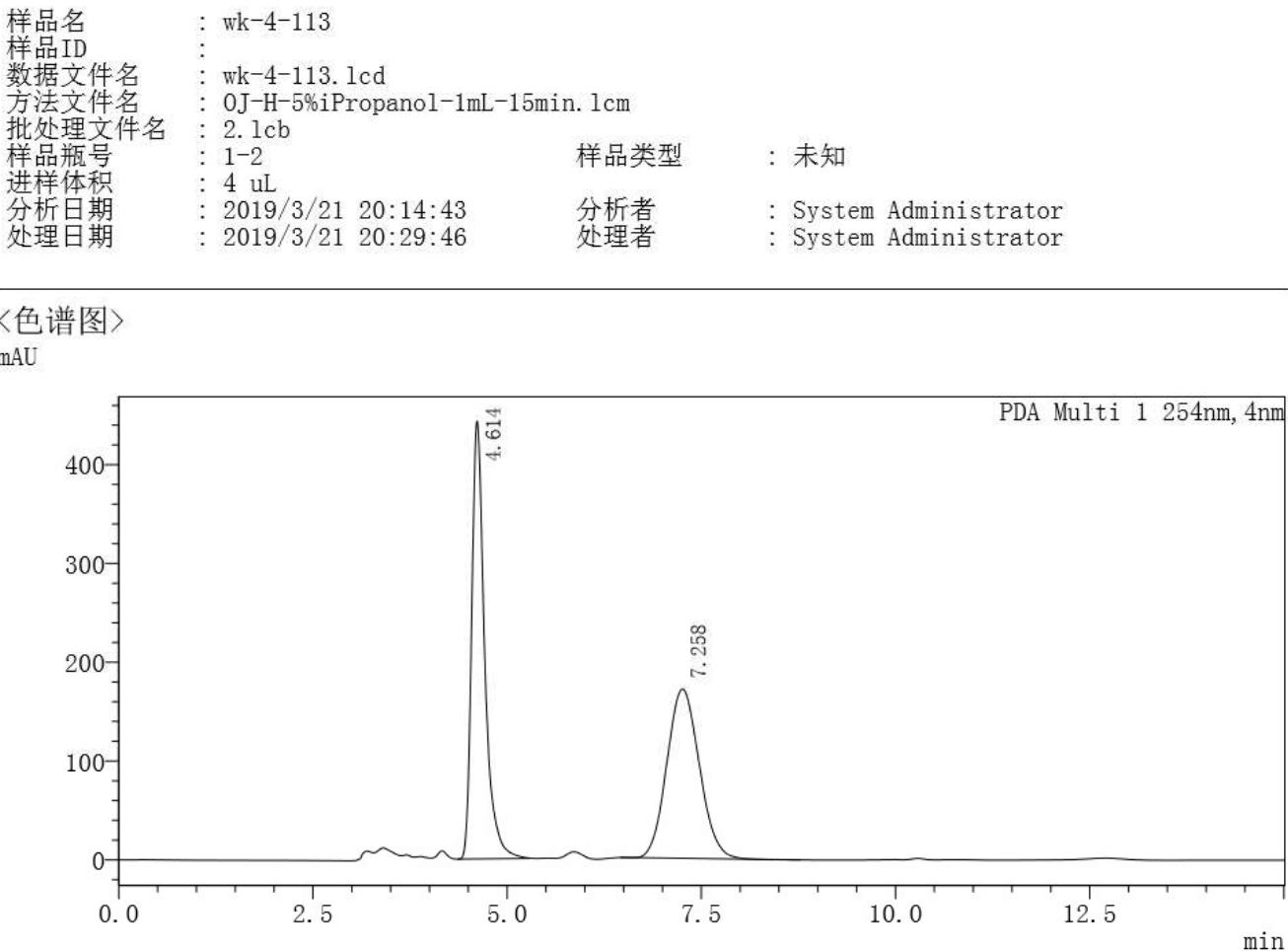

〈峰表〉

PDA Ch1 254nm

\begin{tabular}{|c|c|c|c|c|c|c|c|}
\hline \begin{tabular}{|l|} 
峰号 \\
\end{tabular} & 保留时间 & 面积 & 高度 & 浓度 & 浓度单位 & 标记 & 化合物名 \\
\hline 1 & 4.614 & 5131272 & 443275 & 0.000 & & M & \\
\hline$\sqrt{2}$ & 个. 7.258 & 4986109 & 个 171298 & 0.000 & & M & \\
\hline 总计 & & 10117381 & 614573 & & & & \\
\hline
\end{tabular}

peak number

area

retention time 


\section{in SHMADZu LabSolutions 分析报告}

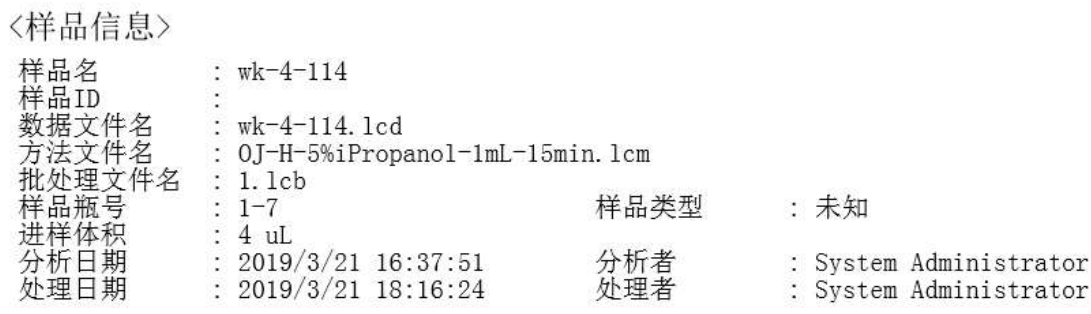

〈色谱图〉

$\mathrm{mAU}$

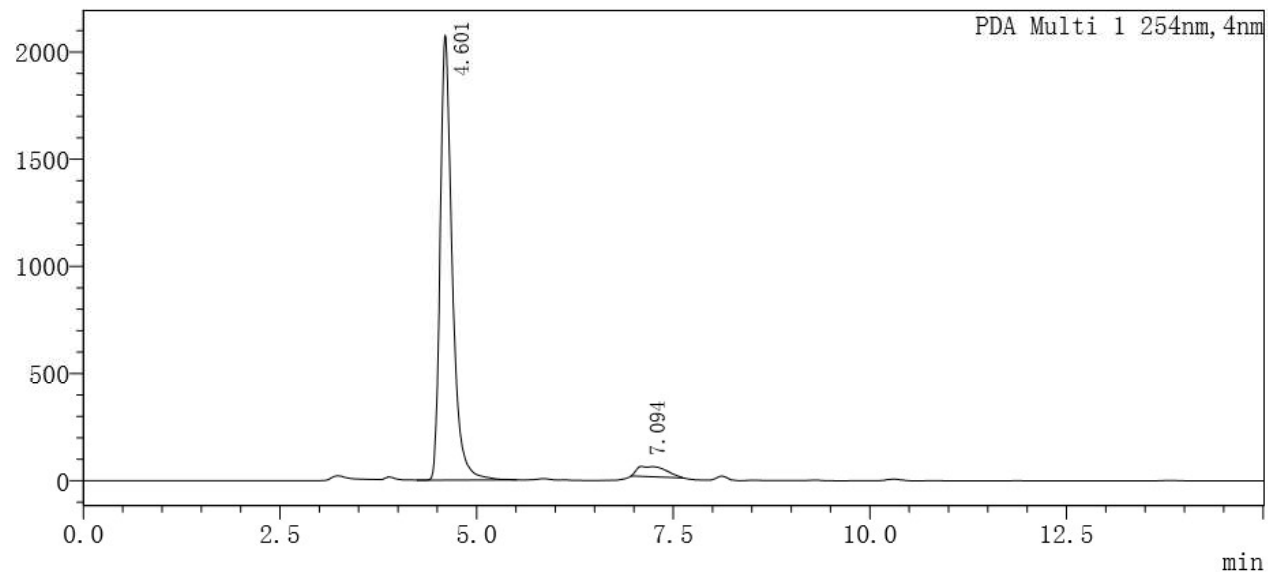

〈峰表〉

\begin{tabular}{|c|c|c|c|c|c|c|c|}
\hline 峰号 & 保留时间 & 面积 & 高度 & 浓度 & 浓度单位 & 标记 & 化合物名 \\
\hline $\begin{array}{ll}1 & 1 \\
\end{array}$ & 4.601 & 21977462 & 2076688 & 0.000 & & M & \\
\hline \begin{tabular}{|l|}
12 \\
\end{tabular} & 7.094 & 不 1216170 & 46556 & 0.000 & & M & \\
\hline 总计 & & 23193632 & 2123244 & & & & \\
\hline numbe & & & ight & & & & \\
\hline
\end{tabular}

retention time 
(3aR,8bS)-3-(4-chlorophenyl)-2,4,8b-trimethyl-1,3a,4,8b-tetrahydrocyclopenta[b]indole $(8 \mathrm{ae})$

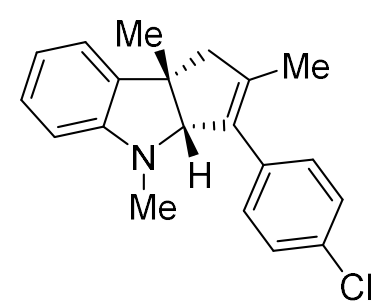

Chemical Formula: $\mathrm{C}_{20} \mathrm{H}_{20} \mathrm{CIN}$

Exact Mass: 309.1284

8ae was prepared according to general procedure 2.3 using $1 \mathrm{a}$ and $7 \mathrm{e}$ and was purified by silica gel column chromatography (petroleum ether/toluene 2:1-1:99) to obtain 8ae as colorless oil $\left(61 \%\right.$ yield). ${ }^{1} \mathrm{H}$ NMR $\left(400 \mathrm{MHz}, \mathrm{CDCl}_{3}\right) \delta 7.38(\mathrm{~d}, J=8.5 \mathrm{~Hz}, 2 \mathrm{H}), 7.32-7.22$ (m, 2H), 7.17-7.03 (m, 2H), 6.70 (td, $J=7.4,0.9 \mathrm{~Hz}, 1 \mathrm{H}), 6.37$ (d, $J=7.5 \mathrm{~Hz}, 1 \mathrm{H}), 4.49$ (s, 1H), $2.91(\mathrm{~d}, J=16.4 \mathrm{~Hz}, 1 \mathrm{H}), 2.63(\mathrm{~d}, J=16.8 \mathrm{~Hz}, 1 \mathrm{H}), 2.56(\mathrm{~s}, 3 \mathrm{H}), 1.71(\mathrm{~s}, 3 \mathrm{H})$, $1.54(\mathrm{~s}, 3 \mathrm{H}) ;{ }^{13} \mathrm{C}$ NMR $\left(101 \mathrm{MHz}, \mathrm{CDCl}_{3}\right) \delta 151.0,138.9,137.6,136.5,134.9,132.3$, 129.9, 128.5, 127.9, 122.5, 117.0, 106.5, 86.8, 53.9, 49.6, 35.5, 28.1, 15.0; HRMS: (ESI) calcd for $\mathrm{C}_{20} \mathrm{H}_{21} \mathrm{CIN}^{+}[\mathrm{M}+\mathrm{H}]^{+} 310.1357$; found 310.1358 .

The enantiomeric purity was established by HPLC analysis using a chiral column: OD-H column, $30^{\circ} \mathrm{C}, n$-Hexane $/ i$-Propanol $=99 / 1$ as eluent, $254 \mathrm{~nm}, 0.5 \mathrm{~mL} / \mathrm{min}$. $\mathrm{tR}=9 \mathrm{~min}$ (minor), 10 min (major).

Optical Rotation: $[\alpha]_{D^{27}}-14.8$ (c 0.4 , $\mathrm{PrOH}$ ) for $88 \%$ ee. 


\section{LabSolutions 分析报出}

〈样品信息〉

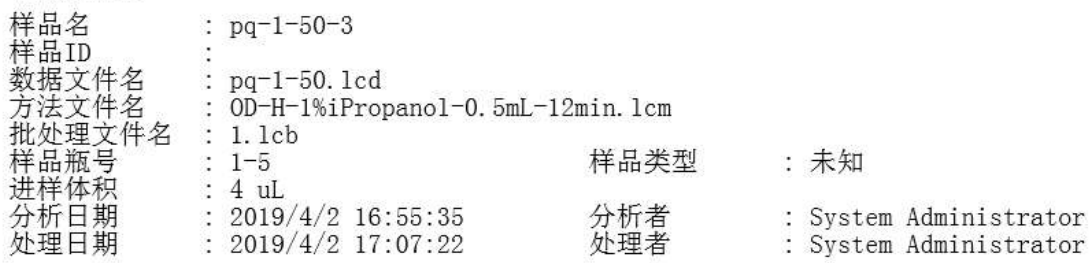

分析者 : System Administrator

处理者 : System Administrator

〈色谱图〉

$\mathrm{mAU}$

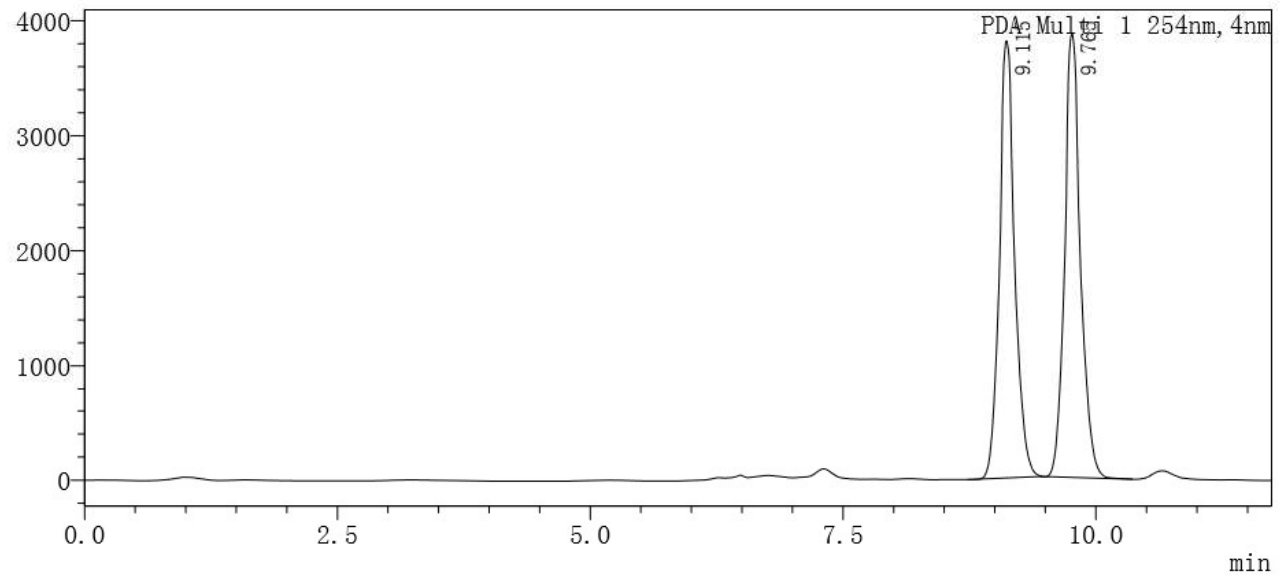

〈峰表〉

PDA Ch1 254nm \begin{tabular}{|l|l|}
\hline 峰号 & 保留时间 \\
\hline
\end{tabular}

\begin{tabular}{|c|c|c|c|c|c|c|c|}
\hline 峰号 & 保留时间 & 面积 & 高度 & 浓度 & 浓度单位 & 标记 & 化合物名 \\
\hline 1 & 9.115 & 39898071 & 3805346 & 0.000 & & M & \\
\hline 2 & 9.765 & 41854456 & 3856353 & 0.000 & & M & \\
\hline 总计 & & 81752527 & 7661698 & & & & \\
\hline
\end{tabular}

peak number

area

\section{retention time}




\section{LabSolutions 分析报出}
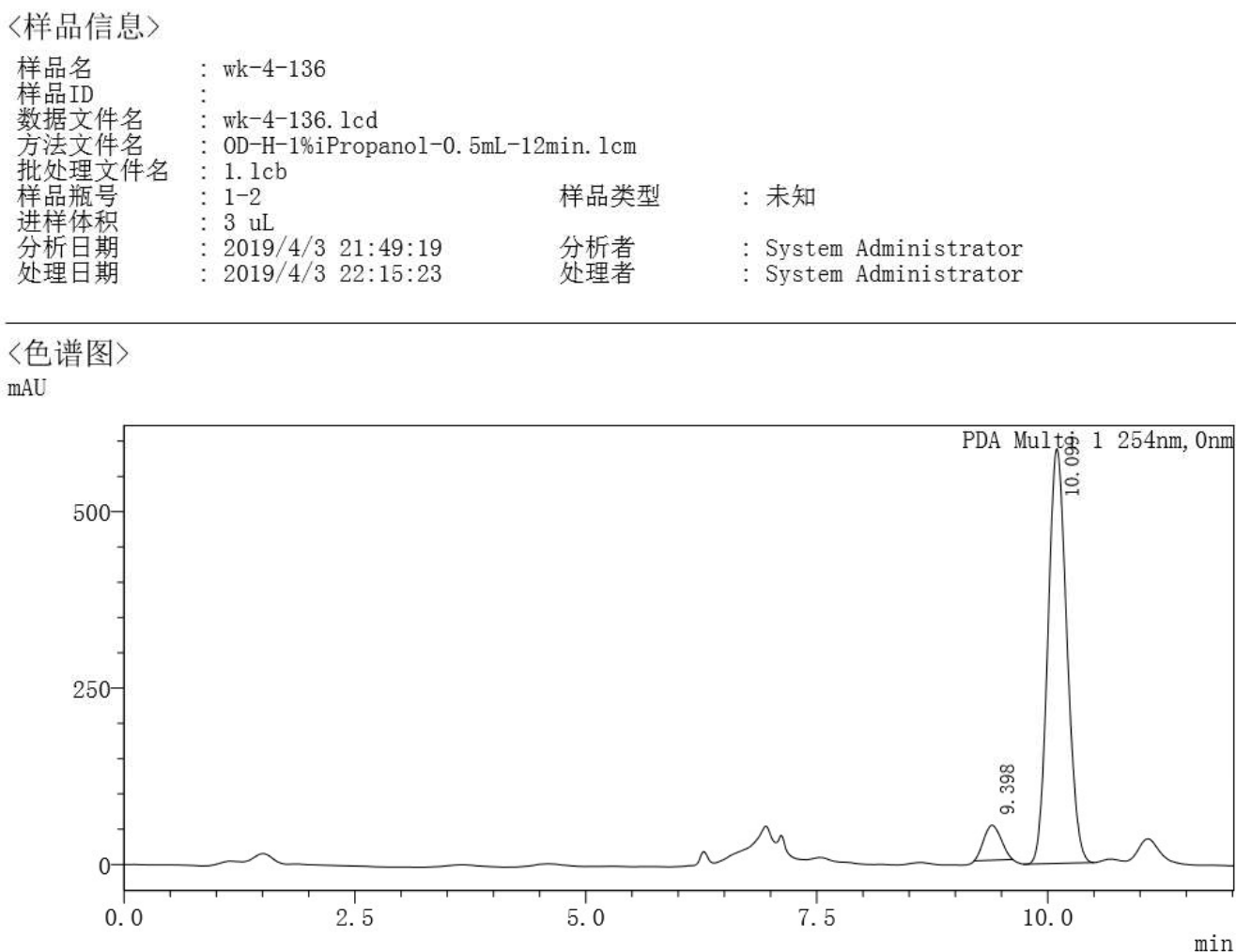

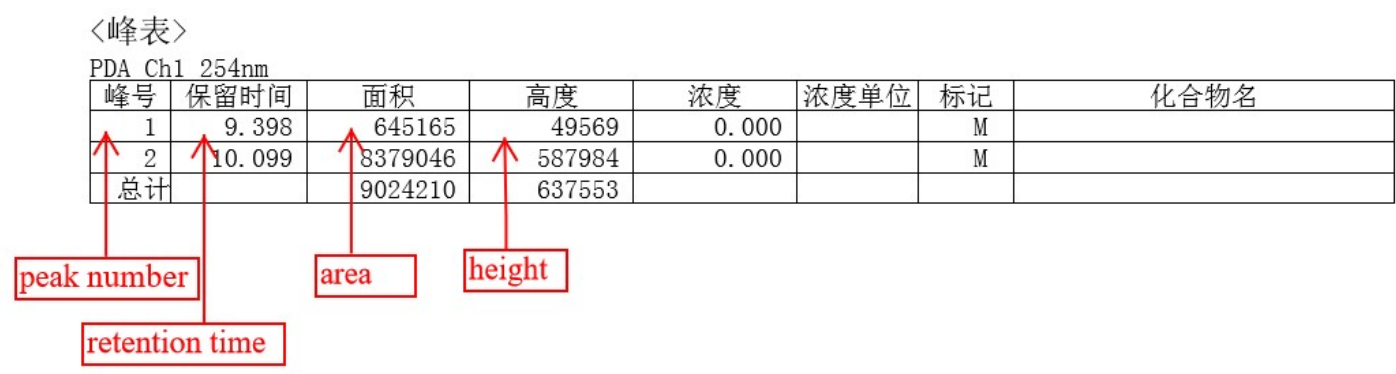


(3aR,8bS)-3-(4-fluorophenyl)-2,4,8b-trimethyl-1,3a,4,8b-tetrahydrocyclopenta[b]indole (8af)

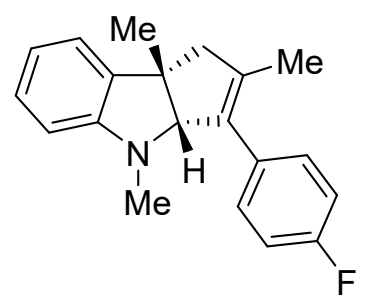

Chemical Formula: $\mathrm{C}_{20} \mathrm{H}_{20} \mathrm{FN}$

Exact Mass: 293.1580

8af was prepared according to general procedure 2.3 using $1 \mathbf{a}$ and $7 \mathrm{f}$ and was purified by silica gel column chromatography (petroleum ether/toluene 2:1-1:99) to obtain 8af as colorless oil (61\% yield). ${ }^{1} \mathrm{H}$ NMR $\left(400 \mathrm{MHz}, \mathrm{CDCl}_{3}\right) \delta$ 7.35-7.23 (m, 2H), 7.16-6.99 (m, 4H), 6.69 (td, $J=7.4,1.0 \mathrm{~Hz}, 1 \mathrm{H}), 6.36$ (dd, $J=8.2,1.0 \mathrm{~Hz}, 1 \mathrm{H}), 4.46(\mathrm{~s}, 1 \mathrm{H}), 2.89$ (d, $J$ $=16.6 \mathrm{~Hz}, 1 \mathrm{H}), 2.61(\mathrm{~d}, J=16.9 \mathrm{~Hz}, 1 \mathrm{H}), 2.54(\mathrm{~s}, 3 \mathrm{H}), 1.70(\mathrm{~s}, 3 \mathrm{H}), 1.53(\mathrm{~s}, 3 \mathrm{H}) ;{ }^{13} \mathrm{C} \mathrm{NMR}$ $\left(101 \mathrm{MHz}, \mathrm{CDCl}_{3}\right) \delta 161.7(\mathrm{~d}, J=245.5 \mathrm{~Hz}), 151.1,138.3,137.7,135.1,133.9,130.1$ (d, $J=7.7 \mathrm{~Hz}$ ), 127.9, 122.6, 117.0, 115.2 (d, $J=21.2 \mathrm{~Hz}), 106.5,87.0,53.9,49.6,35.5,28.1$, 15.1; $\left.{ }^{19} \mathrm{~F} \mathrm{NMR} \mathrm{(376} \mathrm{MHz,} \mathrm{CDCl}_{3}\right): \delta$-115.9; HRMS: (ESI) calcd for $\mathrm{C}_{20} \mathrm{H}_{21} \mathrm{FN}+[\mathrm{M}+\mathrm{H}]^{+}$ 294.1653; found 294.1656.

The enantiomeric purity was established by HPLC analysis using a chiral column: OJ-H column, $30{ }^{\circ} \mathrm{C}, n$-Hexane/i-Propanol $=95 / 5$ as eluent, $254 \mathrm{~nm}, 1 \mathrm{~mL} / \mathrm{min}$. $\mathrm{tR}=5 \mathrm{~min}$ (major), 7 min (minor).

Optical Rotation: $[\alpha]_{D^{27}}-78.0$ (c 0.4 , $\left.{ }^{\mathrm{P}} \mathrm{POH}\right)$ for $90 \%$ ee. 


\section{Lil SHMADZu}

〈样品信息〉

$\begin{array}{llll}\text { 样品名 } & \vdots \text { pq-1-49 } & \\ \text { 样品ID } & \vdots & \\ \text { 数据文件名 } & \vdots \text { pq-1-49. lcd } & \\ \text { 方法文件名 } & \vdots \text { OJ-H-5\% Propanol-1mL-15min. 1cm } & \\ \text { 批处理文件名 } & \vdots 2.1 \mathrm{cb} & & \\ \text { 样品瓶号 } & \vdots 1-6 & \text { 样品类型 } & \text { : 未知 } \\ \text { 进样体积 } & \vdots 4 \mathrm{uL} & & \\ \text { 分析旦期 } & \vdots 2019 / 4 / 215: 20: 55 & \text { 分析者 } & \text { : System Administrator } \\ \text { 处理日期 } & : 2019 / 4 / 215: 35: 57 & \text { 处理者 } & \text { : System Administrator }\end{array}$

〈色谱图〉

$\mathrm{mAU}$

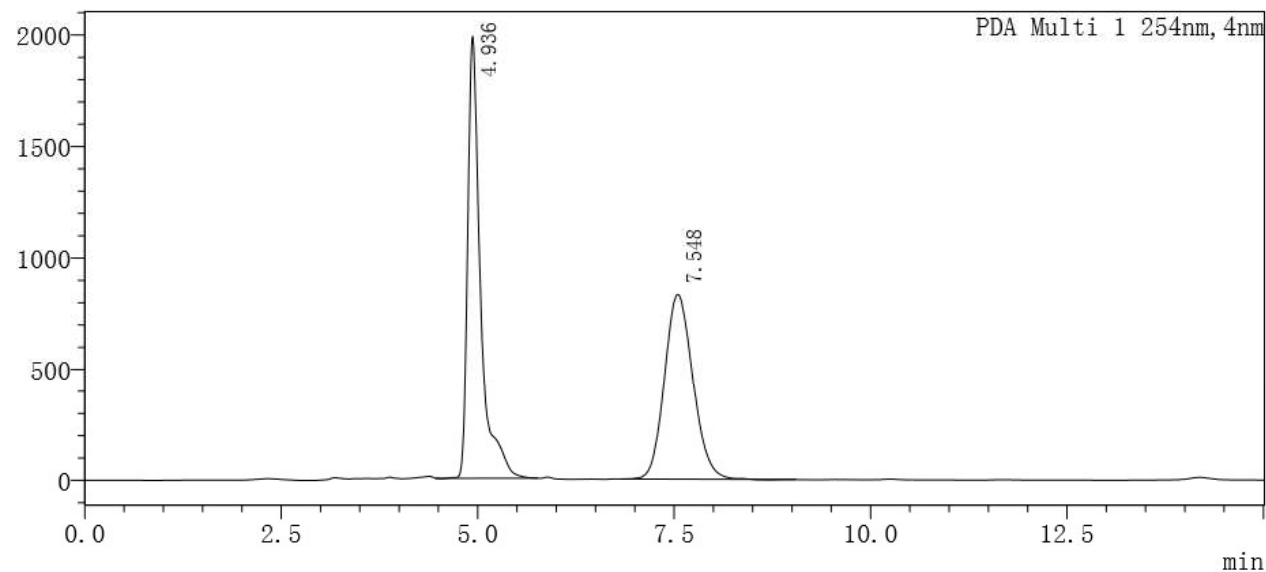

〈峰表〉

PDA Ch1 254nm

\begin{tabular}{|l|l|l|}
\hline 峰号 & 保留时间 & 面积 \\
\hline 1
\end{tabular}

\begin{tabular}{|c|c|c|c|}
\hline 峰号 & 保留时间 & 面积 & 高度 \\
\hline 1 & 4. 936 & \22498116 & 1985846 \\
\hline 2 & 木7.548 & 20964536 & 832253 \\
\hline
\end{tabular}

\begin{tabular}{|c|c|c|c|}
\hline 2 & 林 7.548 & 20964536 & \\
\hline 总计 & & 43462651 & 28180 \\
\hline
\end{tabular}

\begin{tabular}{|l|l|l|r|} 
总计 & & 43462651 & 2818099 \\
\hline
\end{tabular}

peak number

area

0.000

\begin{tabular}{c|c}
\hline 0.000 & 浓度 \\
\hline 0.000 & \\
&
\end{tabular}

height

retention time 


\section{〈样品信息〉}
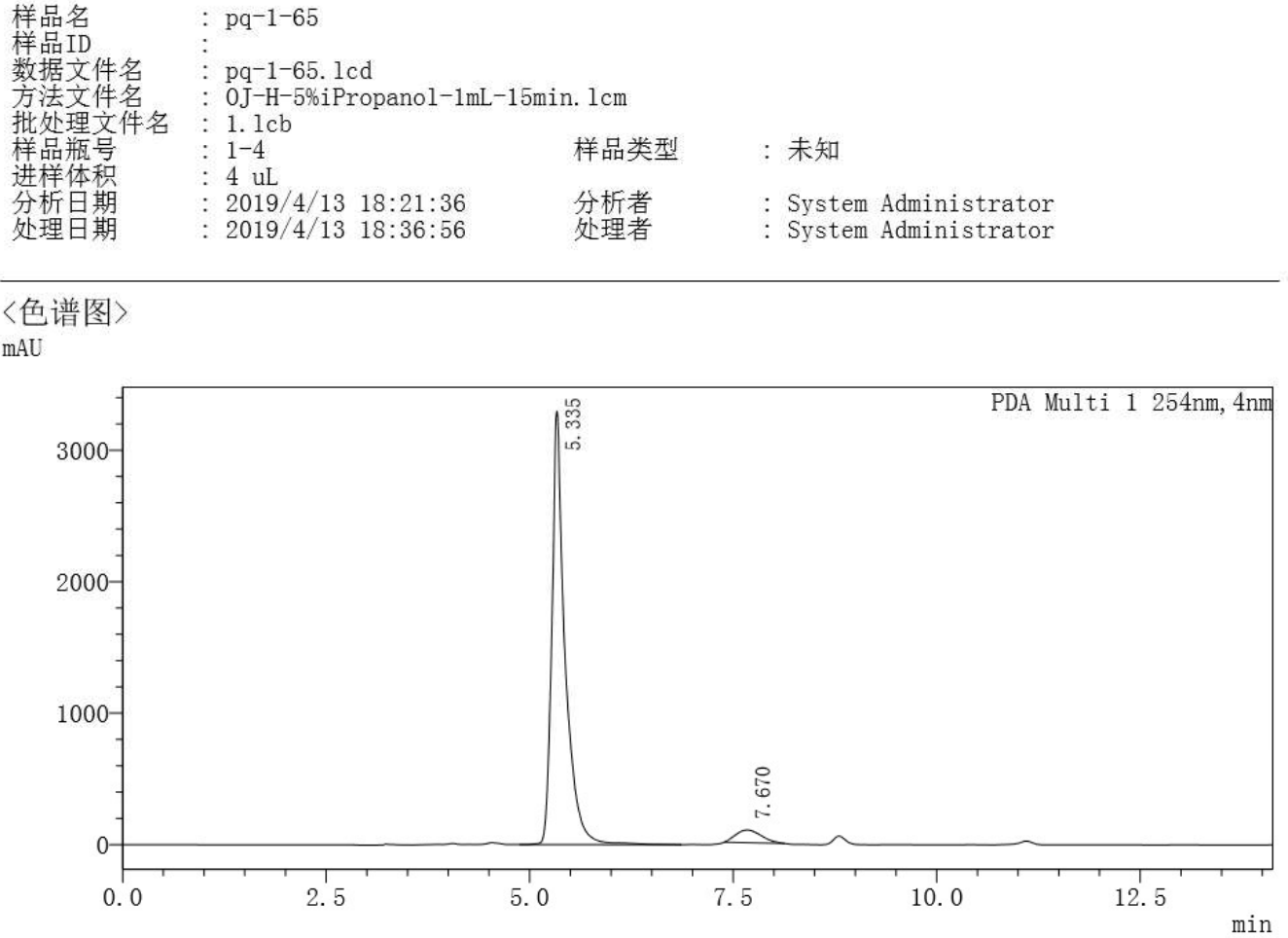

\begin{tabular}{|c|c|c|c|c|c|c|c|}
\hline 峰号 & 保留时间 & 面积 & 高度 & 浓度 & 浓度单位 & 标记 & 化合物名 \\
\hline & 5.335 & 36832790 & 3296371 & 0.000 & & M & \\
\hline 2 & 7.670 & 2087609 & 96116 & 0.000 & & M & \\
\hline 总计 & & 38920398 & 3392488 & & & & \\
\hline
\end{tabular}

retention time 
(3aR,8bS)-2,4,8b-trimethyl-3-(4-(trifluoromethyl)phenyl)-1,3a,4,8b-

tetrahydrocyclopenta[b]indole (8ag)

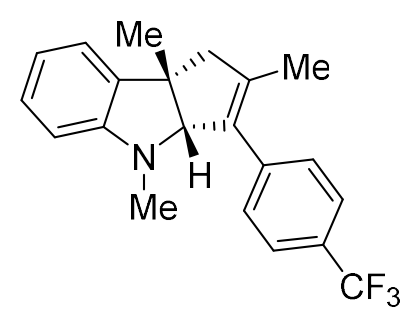

Chemical Formula: $\mathrm{C}_{21} \mathrm{H}_{20} \mathrm{~F}_{3} \mathrm{~N}$

Exact Mass: 343.1548

8ag was prepared according to general procedure 2.3 using $1 \mathrm{a}$ and $\mathbf{7 g}$ and was purified by silica gel column chromatography (petroleum ether/toluene 2:1-1:99) to obtain 8ag as colorless oil (43\% yield). ${ }^{1} \mathrm{H}$ NMR $\left(400 \mathrm{MHz}, \mathrm{CDCl}_{3}\right) \delta 7.63(\mathrm{~d}, J=7.7 \mathrm{~Hz}, 2 \mathrm{H}), 7.41(\mathrm{~d}, J$ $=7.8 \mathrm{~Hz}, 2 \mathrm{H}), 7.09(\mathrm{td}, J=6.9,1.4 \mathrm{~Hz}, 2 \mathrm{H}), 6.68(\mathrm{td}, J=7.4,1.0 \mathrm{~Hz}, 1 \mathrm{H}), 6.34(\mathrm{~d}, J=8.3$ $\mathrm{Hz}, 1 \mathrm{H}), 4.51$ (s, 1H), 2.90 (d, $J=16.7 \mathrm{~Hz}, 1 \mathrm{H}), 2.63$ (d, J = $17.1 \mathrm{~Hz}, 1 \mathrm{H}), 2.51$ (s, 3H), $1.70(\mathrm{~d}, J=1.2 \mathrm{~Hz}, 3 \mathrm{H}), 1.52(\mathrm{~s}, 3 \mathrm{H}) ;{ }^{13} \mathrm{C}$ NMR $\left(101 \mathrm{MHz}, \mathrm{CDCl}_{3}\right) \delta$ 150.9, 141.9, 139.9, 137.5, 134.9, 128.8, 127.9, 127.0 (q, $J=280.3 \mathrm{~Hz}$ ),125.2, 125.1, 122.5, 117.1, 106.5, 86.7, 53.9, 49.7, 35.5, 29.7, 28.0, 15.0; HRMS: (ESI) calcd for $\mathrm{C}_{21} \mathrm{H}_{21} \mathrm{~F}_{3} \mathrm{~N}^{+}[\mathrm{M}+\mathrm{H}]^{+}$ 344.1611 ; found 344.1621 .

The enantiomeric purity was established by HPLC analysis using a chiral column: OD-H column, $30{ }^{\circ} \mathrm{C}, n$-Hexane/i-Propanol $=98 / 2$ as eluent, $254 \mathrm{~nm}, 1 \mathrm{~mL} / \mathrm{min}$. tR $=4.2 \mathrm{~min}$ (minor), 4.6 min (major).

Optical Rotation: [a] $\mathrm{D}^{27}-2.9$ (c 0.4 , ${ }^{\mathrm{P}} \mathrm{POH}$ ) for $85 \%$ ee. 


\section{LabSolutions 分析报告}

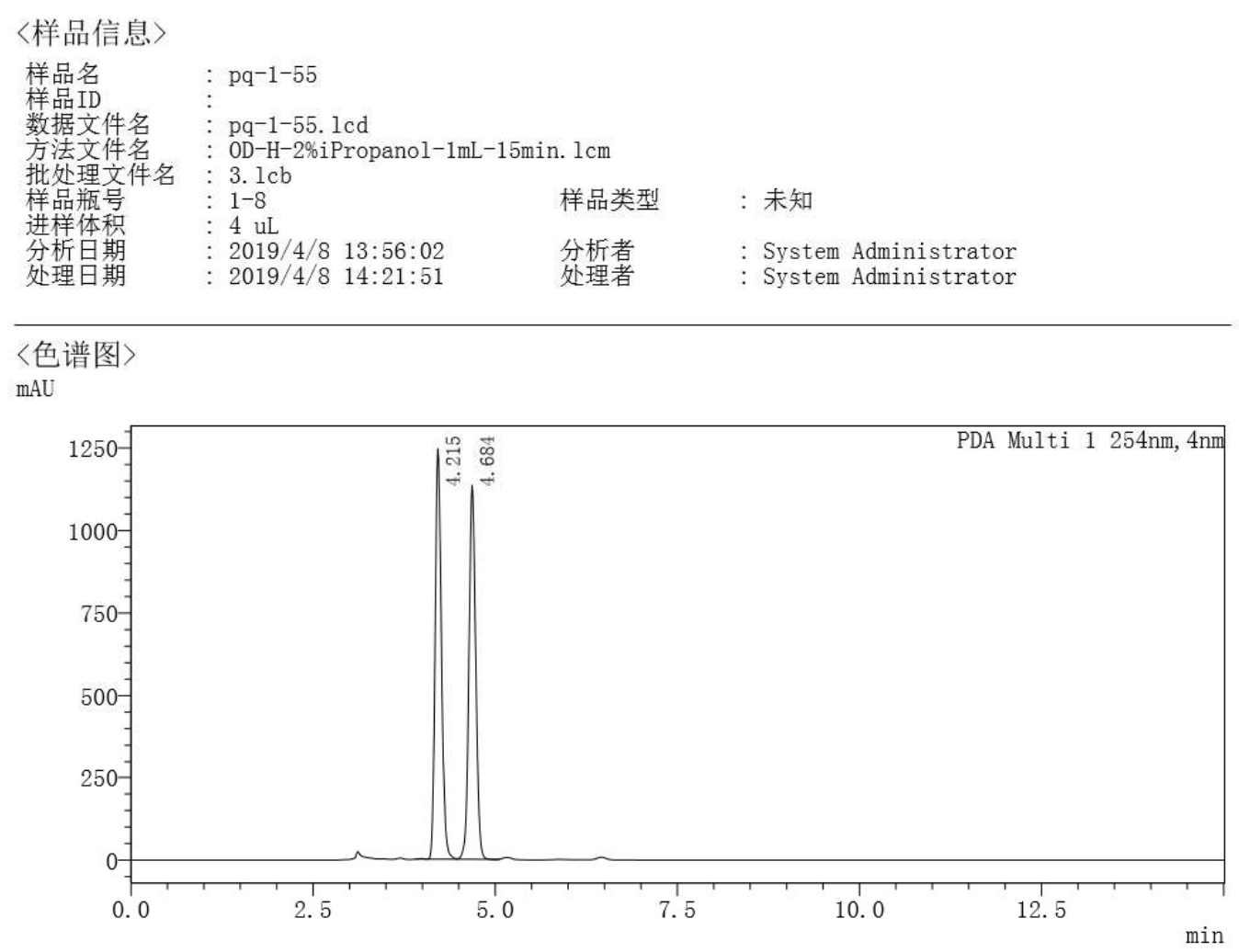

〈峰表〉

\begin{tabular}{|c|c|c|c|c|c|c|c|}
\hline 峰号 & 保留时间 & 面积 & 高度 & 浓度 & 浓度单位 & 标记 & 化合物名 \\
\hline 1 & 4.215 & 17437936 & 1245330 & 0.000 & & M & \\
\hline 2 & 4.684 & 7357853 & 1134811 & 0.000 & & V M & \\
\hline 总计 & & 14795789 & 2380141 & & & & \\
\hline
\end{tabular}

retention time 
1.11 SHMAbSolutions 分析报告

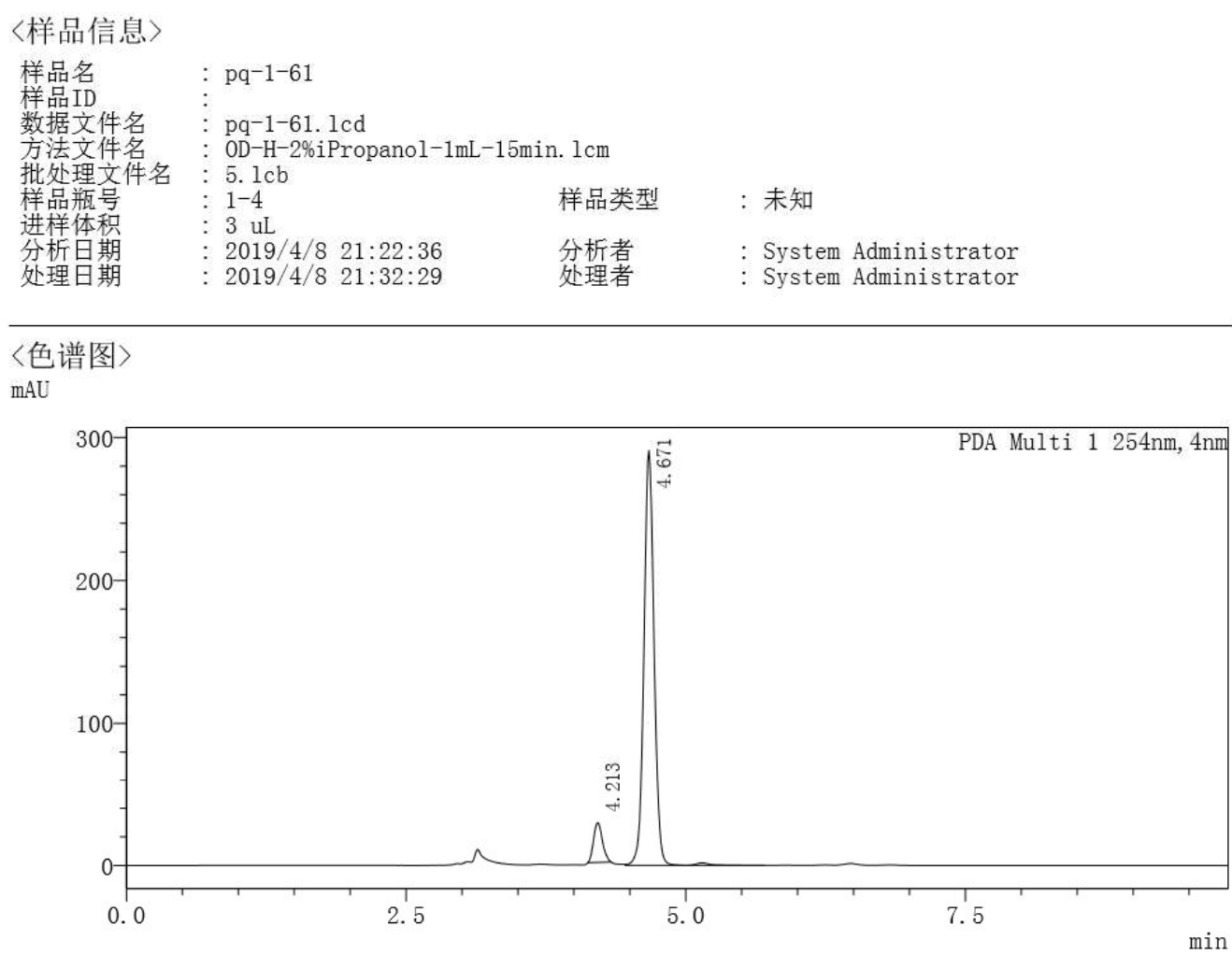

〈峰表〉

\begin{tabular}{|c|c|c|c|c|c|c|c|}
\hline $\begin{array}{l}\text { PDA CI } \\
\text { 峰号 }\end{array}$ & 保留时间 & 面积 & 高度 & 浓度 & 浓度单位 & 标记 & 化合物名 \\
\hline 1 & $\$ 4.213$ & 151670 & $\bigwedge 27879$ & 0.000 & & M & \\
\hline 2 & 4.671 & 1835440 & 290911 & 0.000 & & M & \\
\hline 总计 & & 1987110 & 318790 & & & & \\
\hline mber & & & ht & & & & \\
\hline
\end{tabular}

retention time 
(3aR,8bS)-2,4,8b-trimethyl-3-( $m$-tolyl)-1,3a,4,8b-tetrahydrocyclopenta[b]indole (8ah)

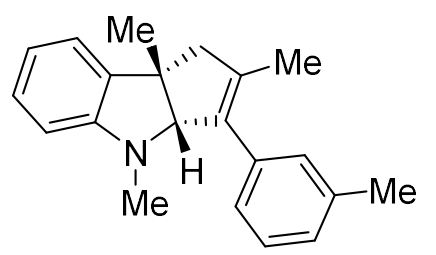

Chemical Formula: $\mathrm{C}_{21} \mathrm{H}_{23} \mathrm{~N}$

Exact Mass: 289.1830

8ah was prepared according to general procedure 2.3 using $1 \mathrm{a}$ and $7 \mathrm{~h}$ and was purified by silica gel column chromatography (petroleum ether/toluene 2:1-1:99) to obtain 8 ah as colorless oil (53\% yield). ${ }^{1} \mathrm{H}$ NMR $\left(400 \mathrm{MHz}, \mathrm{CDCl}_{3}\right) \delta$ 7.25-7.21 (m, 1H), 7.13-7.02 (m, $5 \mathrm{H}), 6.67-6.60(\mathrm{~m}, 1 \mathrm{H}), 6.34-6.28(\mathrm{~m}, 1 \mathrm{H}), 4.47(\mathrm{~s}, 1 \mathrm{H}), 2.86(\mathrm{~d}, J=16.7 \mathrm{~Hz}, 1 \mathrm{H}), 2.56$ (d, $J=16.8 \mathrm{~Hz}, 1 \mathrm{H}), 2.50(\mathrm{~s}, 3 \mathrm{H}), 2.36(\mathrm{~s}, 3 \mathrm{H}), 1.69(\mathrm{~s}, 3 \mathrm{H}), 1.50(\mathrm{~s}, 3 \mathrm{H}) ;{ }^{13} \mathrm{C}$ NMR $(100$ $\left.\mathrm{MHz}, \mathrm{CDCl}_{3}\right) \delta 151.3,138.0,137.9,137.8,136.0,129.3,128.2,127.9,127.4,125.7$, 122.6, 116.9, 106.4, 87.0, 54.1, 49.5, 35.3, 28.1, 21.6, 15.2; HRMS: (ESI) calcd for $\mathrm{C}_{21} \mathrm{H}_{24} \mathrm{~N}^{+}[\mathrm{M}+\mathrm{H}]^{+} 290.1903$; found 290.1921 .

The enantiomeric purity was established by HPLC analysis using a chiral column: OJ-H column, $30^{\circ} \mathrm{C}, n$-Hexane/i-Propanol $=95 / 5$ as eluent, $254 \mathrm{~nm}, 1 \mathrm{~mL} / \mathrm{min}$. tR $=4 \mathrm{~min}$ (major), 5 min (minor).

Optical Rotation: $[\alpha]_{D}{ }^{27}-65.4$ (c 0.4 , $\left.{ }^{i} \mathrm{PrOH}\right)$ for $87 \%$ ee. 


\section{LabSolutions 分析报告}

〈样品信息〉
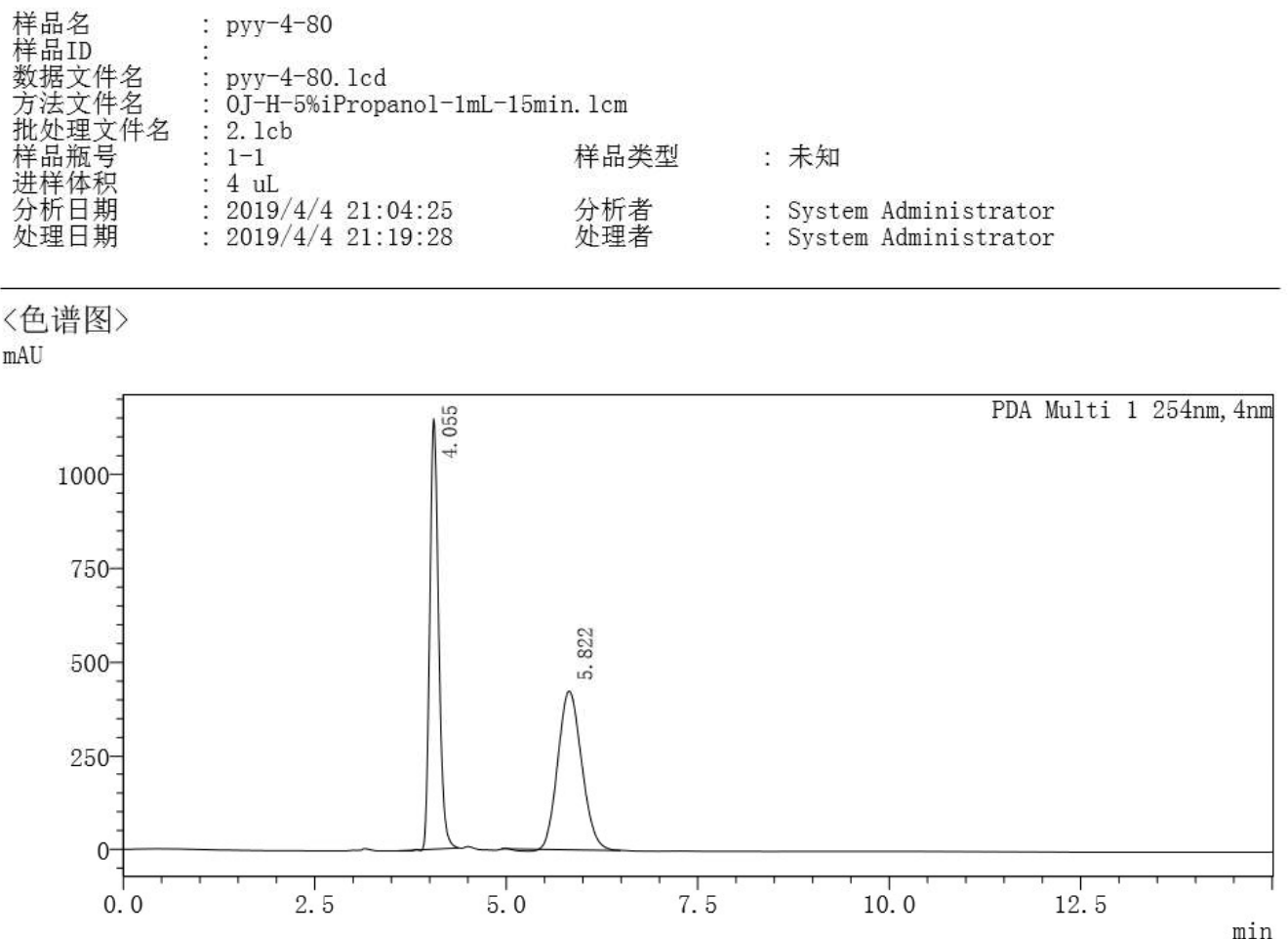

〈峰表〉

\begin{tabular}{|c|c|c|c|c|c|c|c|}
\hline 峰号 & 保留时间 & 面积 & 高度 & 浓度 & 浓度单位 & 标记 & 化合物名 \\
\hline 1 & 4.055 & 爪9206254 & 1147858 & 0.000 & & M & \\
\hline$\uparrow_{2}$ & 估.822 & 9101414 & 424915 & 0.000 & & $\mathrm{M}$ & \\
\hline 总计 & & 18307668 & 1572774 & & & & \\
\hline numbe & & $\frac{1}{\text { area }}$ & height & & & & \\
\hline
\end{tabular}

retention time 


\section{[il SHMADZZ LabSolutions 分析报告}

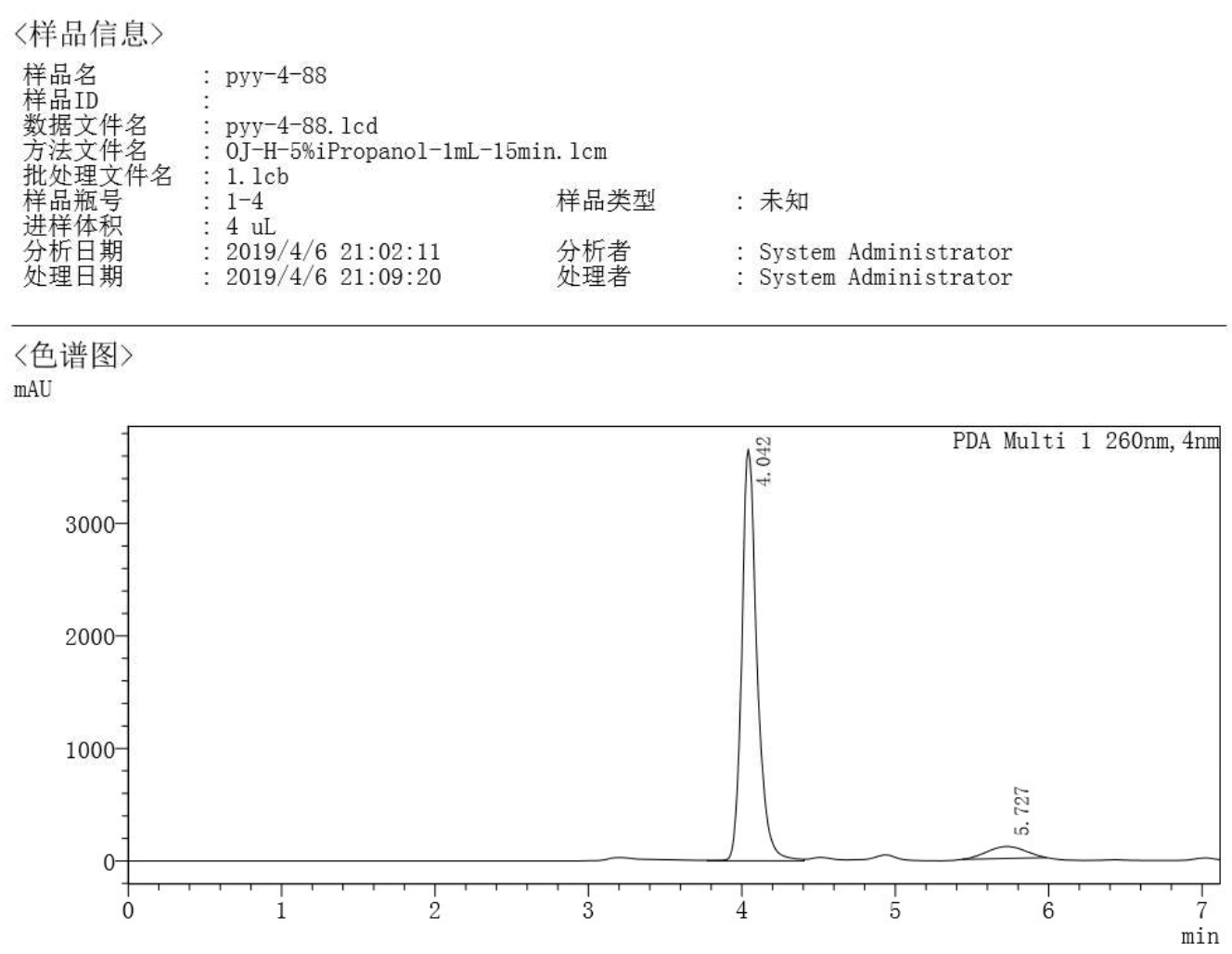

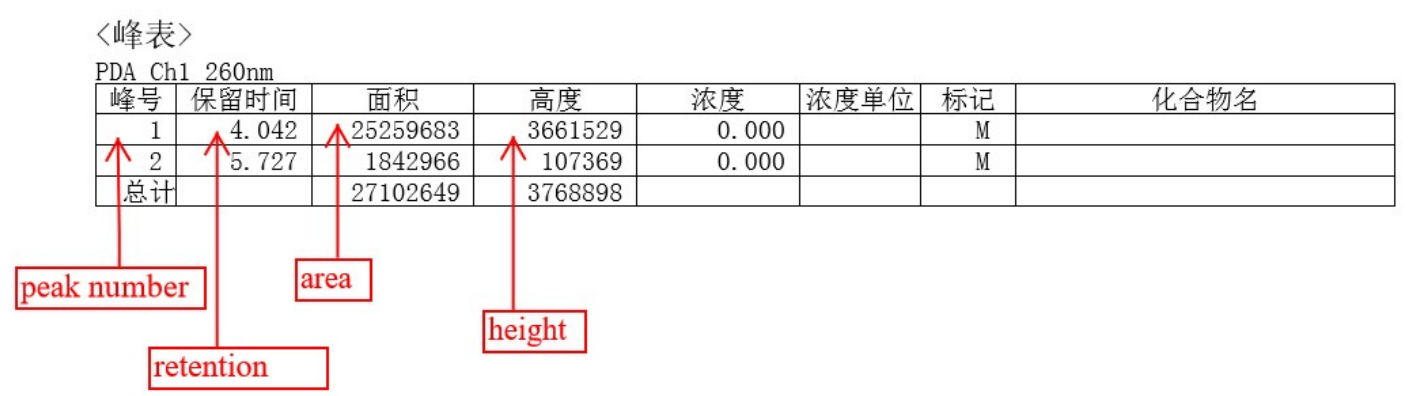


(3aR,8bS)-3-(dibenzo[b, $d]$ thiophen-2-yl)-2,4,8b-trimethyl-1,3a,4,8b-

tetrahydrocyclopenta[b]indole (8ai)

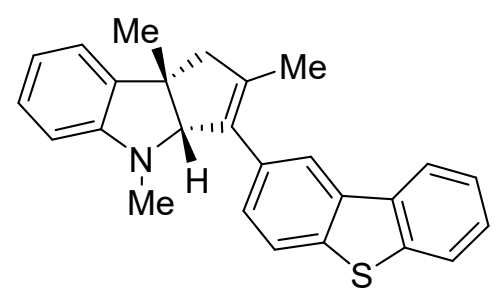

Chemical Formula: $\mathrm{C}_{26} \mathrm{H}_{23} \mathrm{NS}$

Exact Mass: 381.1551

8ai was prepared according to general procedure 2.3 using $1 \mathbf{a}$ and $\mathbf{7} \mathbf{i}$ and was purified by silica gel column chromatography (petroleum ether/toluene 2:1-1:99) to obtain 8ai as colorless oil (45\% yield). ${ }^{1} \mathrm{H}$ NMR $\left(400 \mathrm{MHz}, \mathrm{CDCl}_{3}\right) \delta 8.23-8.15(\mathrm{~m}, 1 \mathrm{H}), 8.11(\mathrm{~d}, J=1.0$ $\mathrm{Hz}, 1 \mathrm{H}), 7.96-7.85(\mathrm{~m}, 2 \mathrm{H}), 7.55-7.48(\mathrm{~m}, 2 \mathrm{H}), 7.45(\mathrm{dd}, J=8.2,1.6 \mathrm{~Hz}, 1 \mathrm{H}), 7.18-7.08$ $(\mathrm{m}, 2 \mathrm{H}), 6.77-6.65(\mathrm{~m}, 1 \mathrm{H}), 6.37(\mathrm{~d}, J=7.7 \mathrm{~Hz}, 1 \mathrm{H}), 4.63(\mathrm{~s}, 1 \mathrm{H}), 2.98(\mathrm{~d}, J=16.6 \mathrm{~Hz}$, $1 \mathrm{H}), 2.69(\mathrm{~d}, J=16.8 \mathrm{~Hz}, 1 \mathrm{H}), 2.54(\mathrm{~s}, 3 \mathrm{H}), 1.80(\mathrm{~s}, 3 \mathrm{H}), 1.61(\mathrm{~s}, 3 \mathrm{H}) ;{ }^{13} \mathrm{C} N M R(101 \mathrm{MHz}$, $\left.\mathrm{CDCl}_{3}\right) \delta 151.1,139.8,138.4,137.7,135.9,135.7,135.5,134.4,127.9,127.3,126.8$, $124.4,122.9,122.60,122.58,121.7,121.6,116.9,106.5,87.2,53.9,49.6,35.5,28.1$, 15.1; HRMS: (ESI) calcd for $\mathrm{C}_{26} \mathrm{H}_{24} \mathrm{NS}^{+}[\mathrm{M}+\mathrm{H}]^{+} 382.1624$; found 382.1631 .

The enantiomeric purity was established by HPLC analysis using a chiral column: AD-H column, $30^{\circ} \mathrm{C}, n$-Hexane $/ i-P r o p a n o l=98 / 2$ as eluent, $254 \mathrm{~nm}, 0.5 \mathrm{~mL} / \mathrm{min}$. $\mathrm{tR}=7 \mathrm{~min}$ (minor), 9 min (major).

Optical Rotation: $[\alpha]_{D^{27}}-86.5$ (c 0.4 , $\mathrm{PrOH}$ ) for $87 \%$ ee. 


\section{LabSolutions 分析报告}

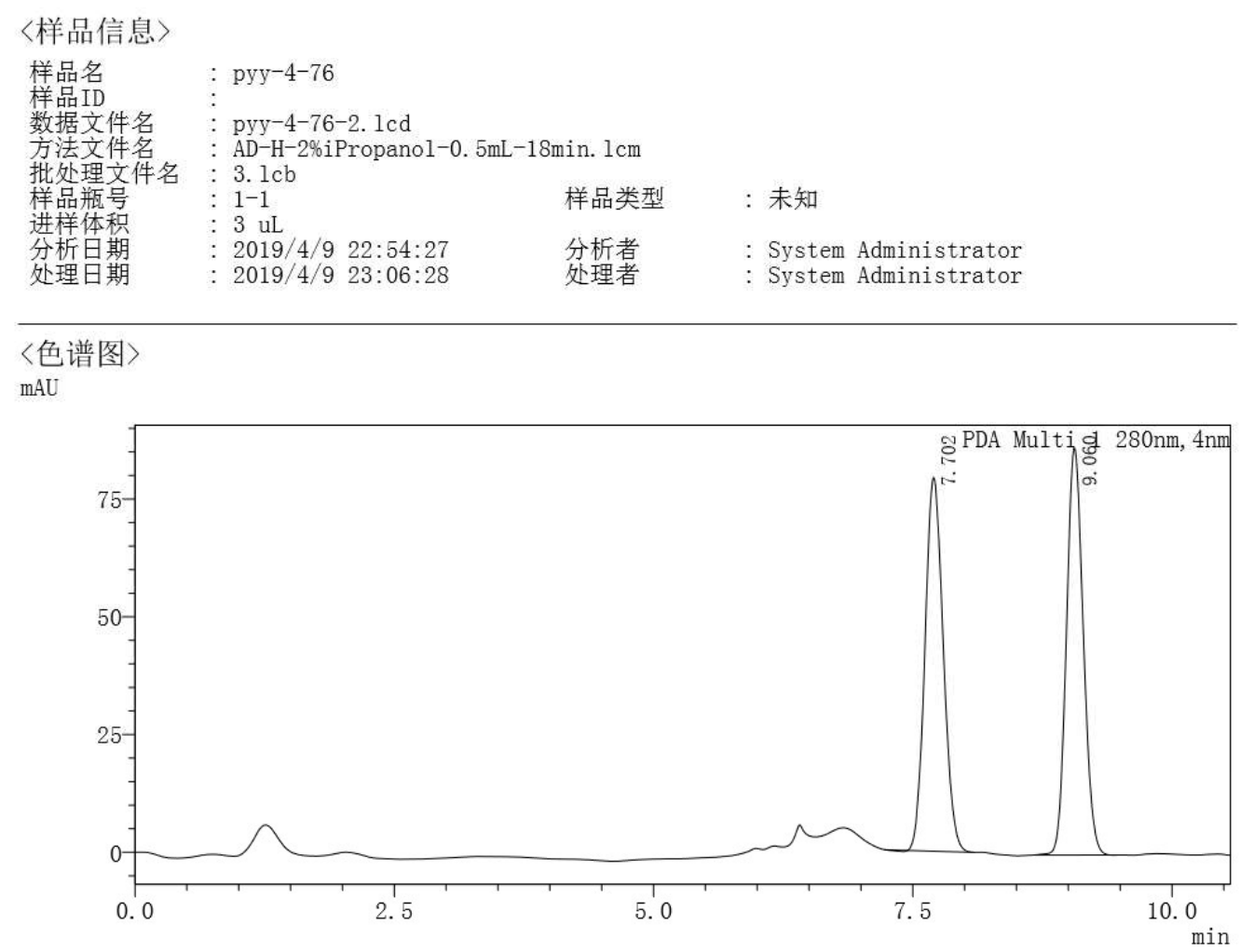

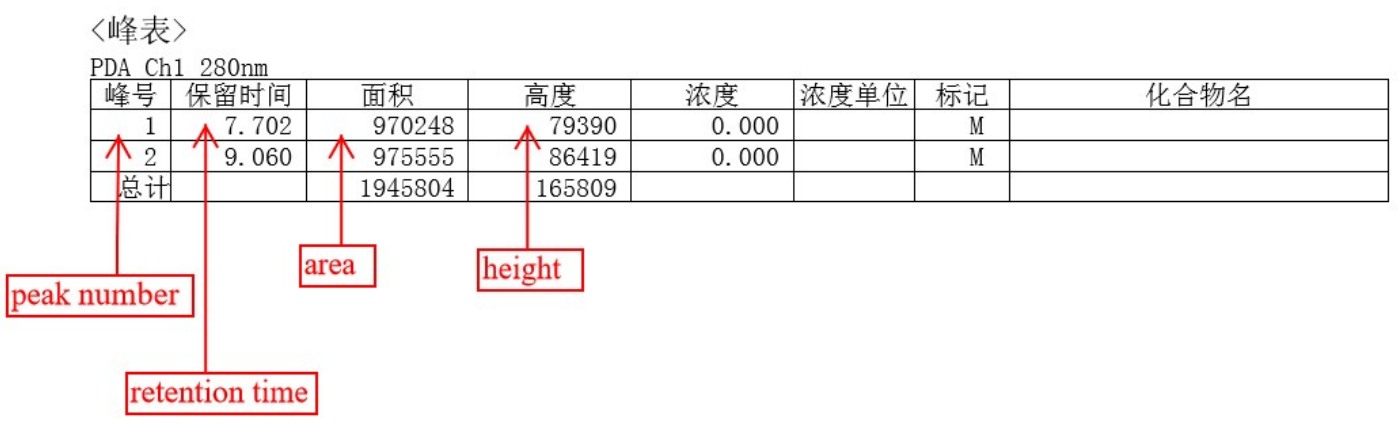




\section{LabSolutions 分析报告}

〈样品信息〉

样品名
样品ID
数据文件名
方法文件名
批处理文件名
样品瓶号
进样体积
分析日期
处理日期

pq-1-64

pq-1-64-2. $1 \mathrm{~cd}$

AD-H-2\%iPropanol-0.5mL-18min. $1 \mathrm{~cm}$

$2.1 \mathrm{cb}$

: $1-1$

样品类型

: 未知

: 2019/4/9 22:41:31

分析者

: System Administrator

2019/4/9 22:53:00 处理者

: System Administrator

〈色谱图〉

$\mathrm{mAU}$

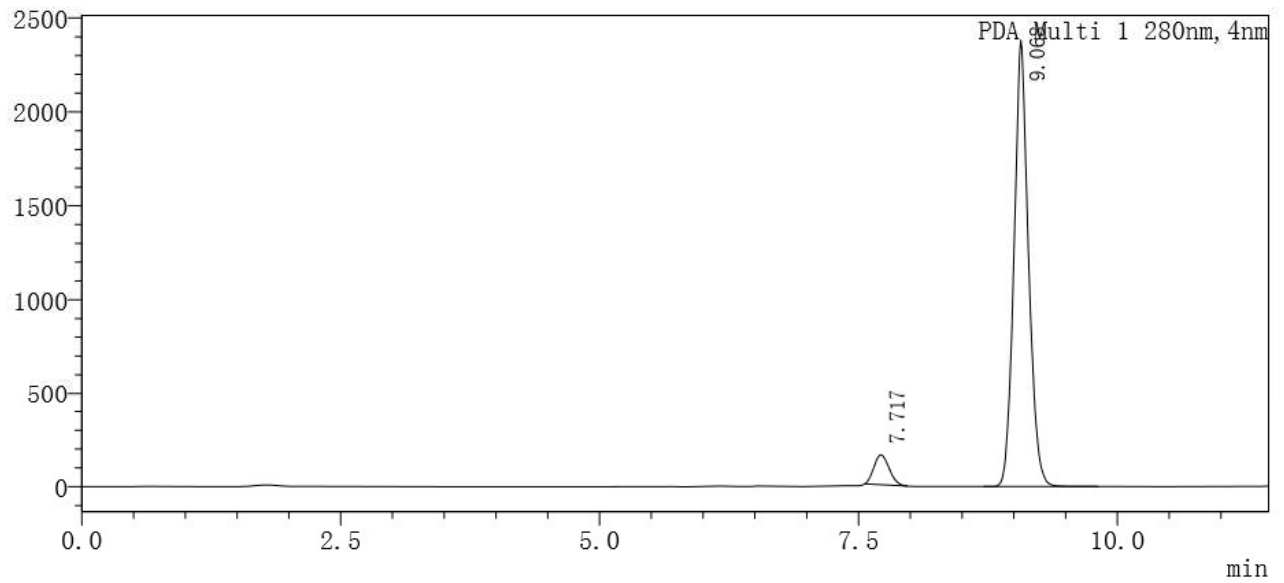

〈峰表〉

\begin{tabular}{|c|c|c|c|c|c|c|c|}
\hline \begin{tabular}{|l|} 
峰号 \\
\end{tabular} & 保留时间 & 面积 & 高度 & 浓度 & 浓度单位 & 标记 & 化合物名 \\
\hline 1 & 7.717 & 1618513 & 158240 & 0.000 & & M & \\
\hline \begin{tabular}{|l|} 
木 2 \\
\end{tabular} & 9.068 & 23059175 & 2381615 & 0.000 & & M & \\
\hline 总计 & & 24677688 & 2539855 & & & & \\
\hline imbe & & & ight & & & & \\
\hline
\end{tabular}

retention time 
(3aR,8bS)-3-(dibenzo[b,d]furan-2-yl)-2,4,8b-trimethyl-1,3a,4,8b-

tetrahydrocyclopenta[b]indole (8aj)

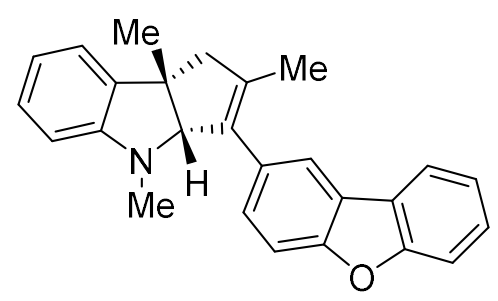

Chemical Formula: $\mathrm{C}_{26} \mathrm{H}_{23} \mathrm{NO}$

Exact Mass: 365.1780

8aj was prepared according to general procedure 2.3 using $\mathbf{1 a}$ and $\mathbf{7 j}$ and was purified by silica gel column chromatography (petroleum ether/toluene 2:1-1:99) to obtain 8aj as white solid (58\% yield). ${ }^{1} \mathrm{H}$ NMR $\left(400 \mathrm{MHz}, \mathrm{CDCl}_{3}\right) \delta$ 7.97-7.93 $(\mathrm{m}, 1 \mathrm{H})$, 7.87-7.84 (m, $1 \mathrm{H})$, 7.62-7.54 (m, 2H), 7.50-7.44 (m, 1H), 7.41-7.32 (m, 2H), 7.12-7.04 (m, 2H), 6.70$6.63(\mathrm{~m}, 1 \mathrm{H}), 6.32(\mathrm{~d}, J=7.7 \mathrm{~Hz}, 1 \mathrm{H}), 4.55(\mathrm{~s}, 1 \mathrm{H}), 2.92(\mathrm{~d}, J=16.6 \mathrm{~Hz}, 1 \mathrm{H}), 2.64(\mathrm{~d}, J$ $=16.8 \mathrm{~Hz}, 1 \mathrm{H}), 2.48(\mathrm{~s}, 3 \mathrm{H}), 1.73(\mathrm{~s}, 3 \mathrm{H}), 1.55(\mathrm{~s}, 3 \mathrm{H}) ;{ }^{13} \mathrm{C} \mathrm{NMR}\left(100 \mathrm{MHz}, \mathrm{CDCl}_{3}\right) \delta$ $156.5,155.0,151.1,137.9,137.7,135.9,132.7,127.8,127.7,127.2,124.23,124.17$, $122.7,122.5,120.7,120.6,116.8,111.7,111.4,106.3,87.3,53.8,49.5,35.3,28.1,15.0$; HRMS: (ESI) calcd for $\mathrm{C}_{26} \mathrm{H}_{24} \mathrm{NO}^{+}[\mathrm{M}+\mathrm{H}]^{+} 366.1852$; found 366.1846 .

The enantiomeric purity was established by HPLC analysis using a chiral column: AD-H column, $30{ }^{\circ} \mathrm{C}, n$-Hexane/i-Propanol $=98 / 2$ as eluent, $254 \mathrm{~nm}, 0.5 \mathrm{~mL} / \mathrm{min}$. $\mathrm{tR}=7.5 \mathrm{~min}$ (minor), 7.9 min (major).

Optical Rotation: $[\alpha]_{D^{27}}-79.7$ (c 0.4 , $\left.{ }^{\mathrm{P}} \mathrm{POH}\right)$ for $88 \%$ ee. 


\section{LabSolutions 分析报告}

〈样品信息〉
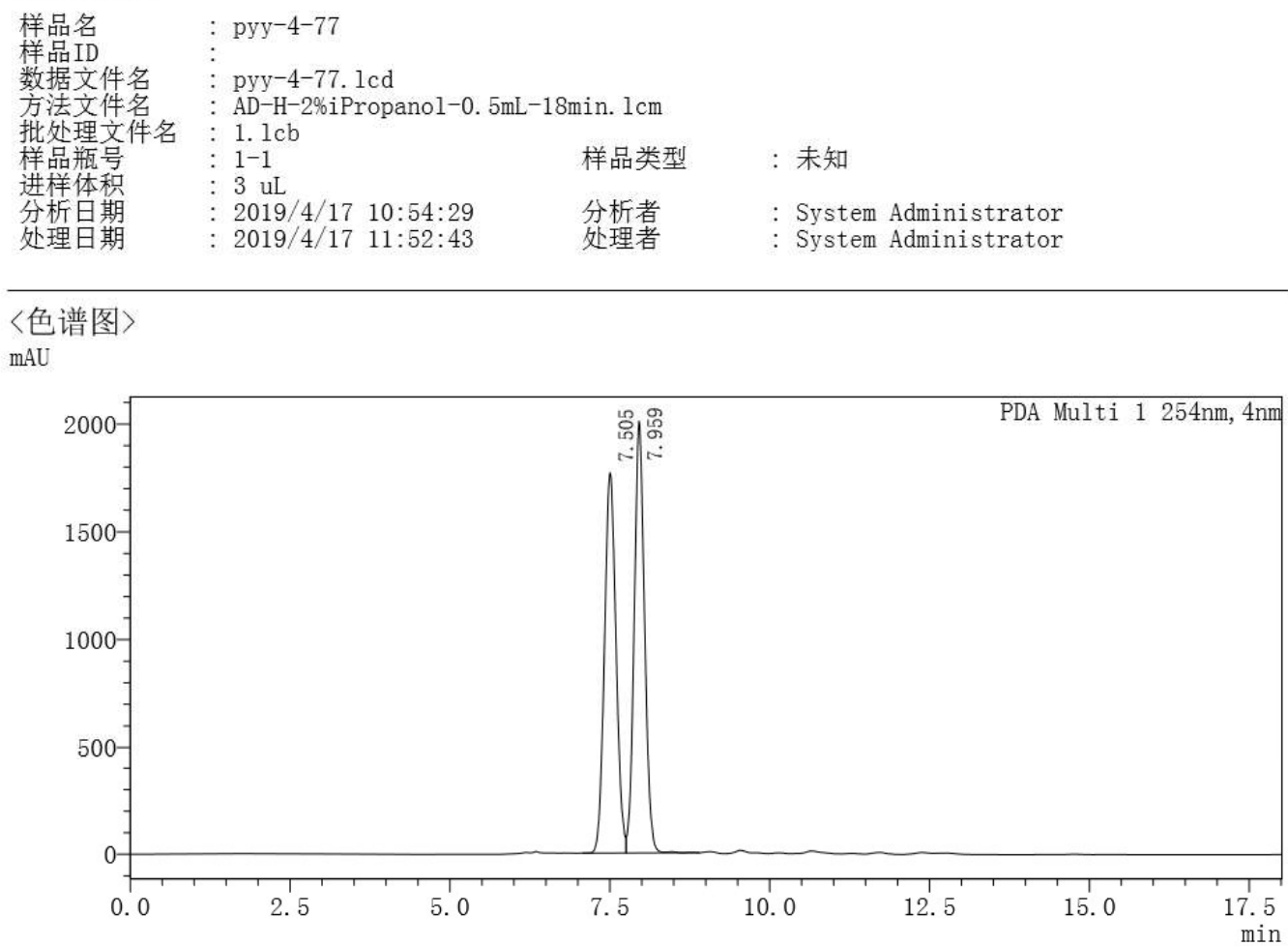

〈峰表〉

PDA Ch1 $254 \mathrm{~nm}$ 峰号

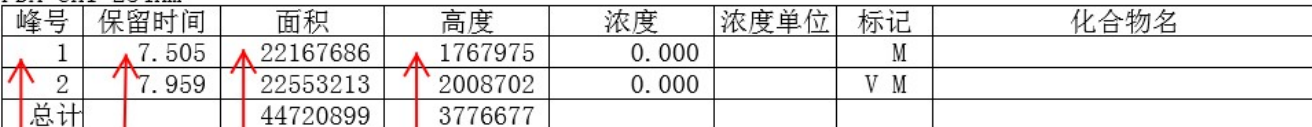

retention time 


\section{LabSolutions 分析报告}

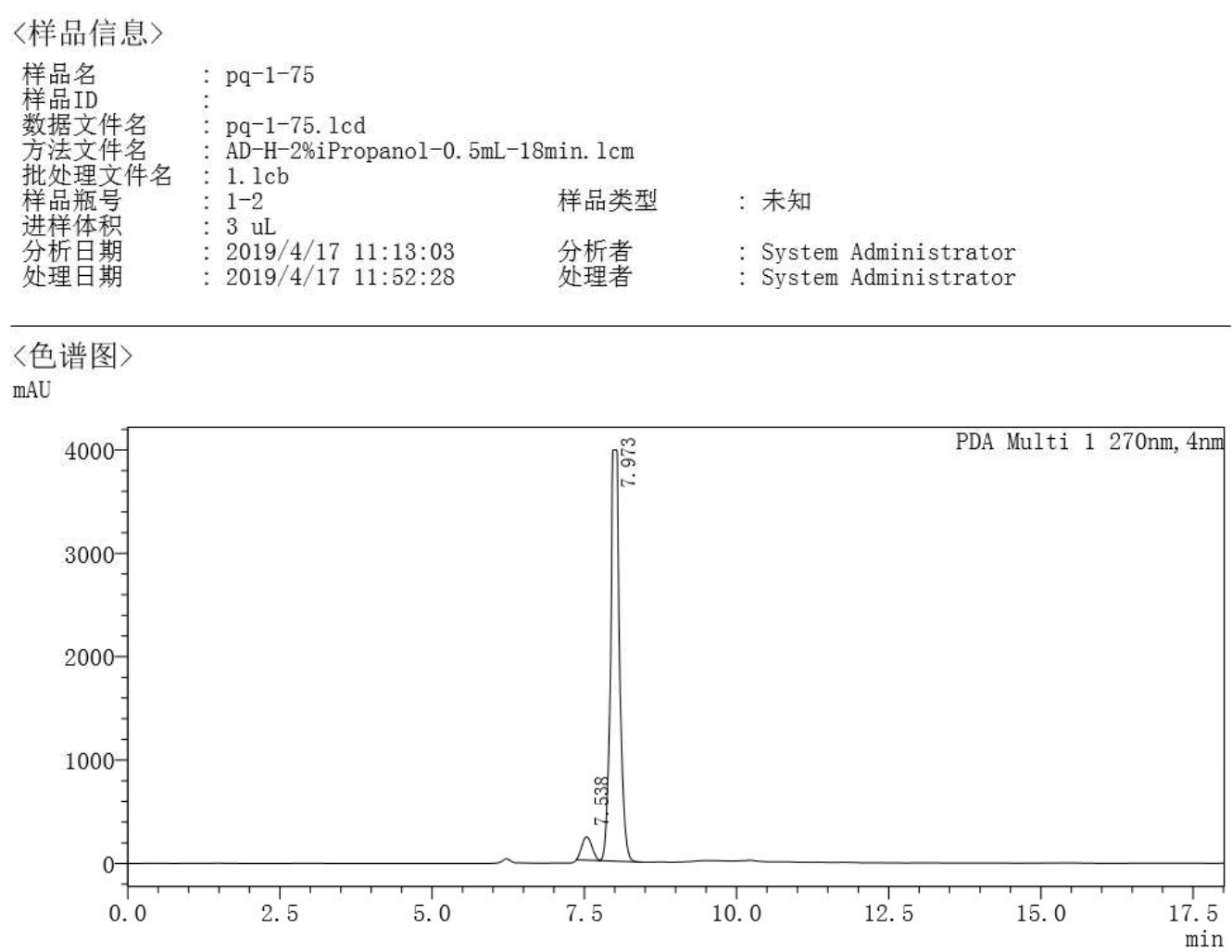

〈峰表〉

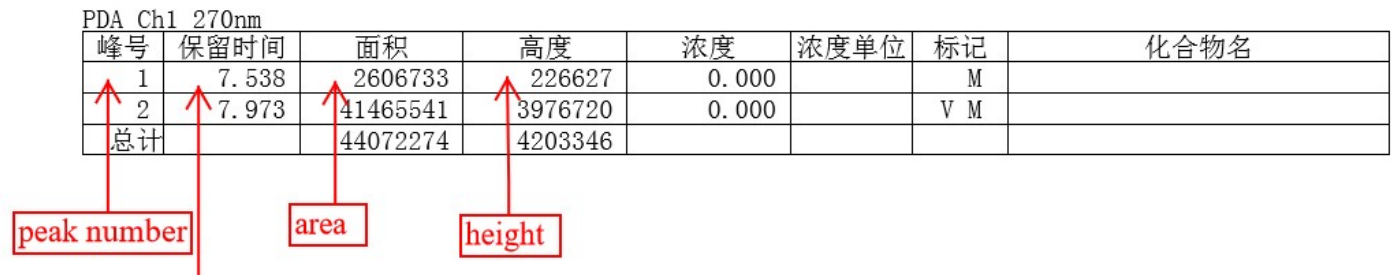

retention time 
(3aR,8bS)-2,4,8b-trimethyl-3-(1-methyl-1H-indol-5-yl)-1,3a,4,8b-

tetrahydrocyclopenta[b]indole (8ak)

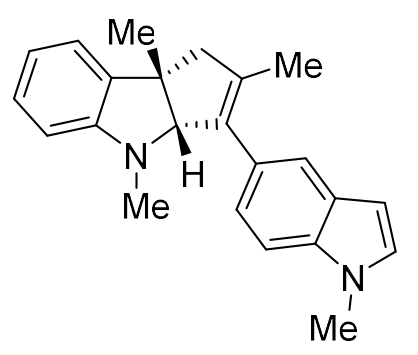

Chemical Formula: $\mathrm{C}_{23} \mathrm{H}_{24} \mathrm{~N}_{2}$

Exact Mass: 328.1939

8ak was prepared according to general procedure 2.3 using $\mathbf{1 a}$ and $7 \mathbf{k}$ and was purified by silica gel column chromatography (petroleum ether/toluene 2:1-1:99) to obtain 8ak as white solid (68\% yield). ${ }^{1} \mathrm{H}$ NMR $\left(400 \mathrm{MHz}, \mathrm{CDCl}_{3}\right) \delta 7.58-7.55(\mathrm{~m}, 1 \mathrm{H}), 7.35(\mathrm{~d}, J=8.4$ $\mathrm{Hz}, 1 \mathrm{H}), 7.21(\mathrm{dd}, J=8.4,1.5 \mathrm{~Hz}, 1 \mathrm{H}), 7.14-7.06(\mathrm{~m}, 3 \mathrm{H}), 6.71-6.64(\mathrm{~m}, 1 \mathrm{H}), 6.50(\mathrm{dd}, J$ $=3.1,0.8 \mathrm{~Hz}, 1 \mathrm{H}), 6.33(\mathrm{~d}, J=7.7 \mathrm{~Hz}, 1 \mathrm{H}), 4.58(\mathrm{~s}, 1 \mathrm{H}), 3.83(\mathrm{~s}, 3 \mathrm{H}), 2.92(\mathrm{dt}, J=16.6$, $1.5 \mathrm{~Hz}, 1 \mathrm{H}), 2.62(\mathrm{~d}, J=16.8 \mathrm{~Hz}, 1 \mathrm{H}), 2.49(\mathrm{~s}, 3 \mathrm{H}), 1.75(\mathrm{~d}, J=1.1 \mathrm{~Hz}, 3 \mathrm{H}), 1.57(\mathrm{~s}, 3 \mathrm{H})$; ${ }^{13} \mathrm{C}$ NMR $\left(100 \mathrm{MHz}, \mathrm{CDCl}_{3}\right) \delta 151.3,136.7,136.4,135.6,129.0,128.4,127.6,122.5$, 122.4, 120.7, 116.6, 108.8, 106.1, 101.0, 87.3, 53.9, 49.3, 35.1, 32.8, 28.0, 15.1; HRMS: (ESI) calcd for $\mathrm{C}_{23} \mathrm{H}_{25} \mathrm{~N}_{2}+[\mathrm{M}+\mathrm{H}]^{+} 329.2012$; found 329.1997.

The enantiomeric purity was established by HPLC analysis using a chiral column: AD-H column, $30{ }^{\circ} \mathrm{C}, n$-Hexane/i-Propanol $=97 / 3$ as eluent, $254 \mathrm{~nm}, 1 \mathrm{~mL} / \mathrm{min}$. $\mathrm{tR}=4 \mathrm{~min}$ (minor), 6 min (major).

Optical Rotation: $[\alpha]^{23}-134.2$ (c $\left.0.1,{ }^{i} \mathrm{PrOH}\right)$ for $88 \%$ ee. 


\section{1]IIIIMADSolutions 分析报告}

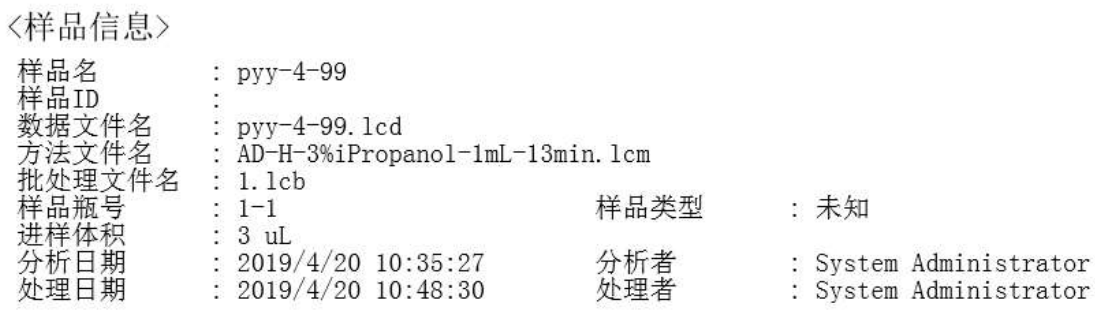

〈色谱图〉

$\mathrm{mAU}$

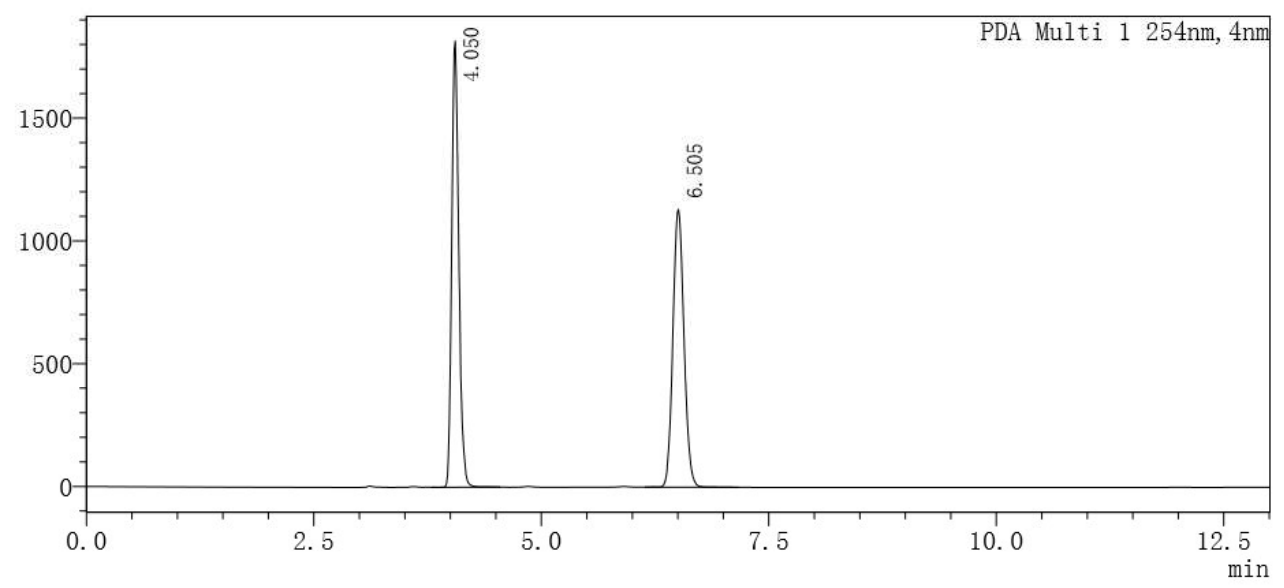

〈峰表〉

\begin{tabular}{|c|c|c|c|c|c|c|c|}
\hline 峰号 & 保留时间 & 面积 & 高度 & 浓度 & 浓度单位 & 标记 & 化合物名 \\
\hline 1 & $\uparrow 4.050$ & 9743856 & 1817916 & 0.000 & & M & \\
\hline 2 & 6.505 & 9585539 & 朴 1132248 & 0.000 & & M & \\
\hline 总计 & & 19329395 & 2950164 & & & & \\
\hline
\end{tabular}

retention time 


\section{SHMMADZu LabSolutions 分析报告}

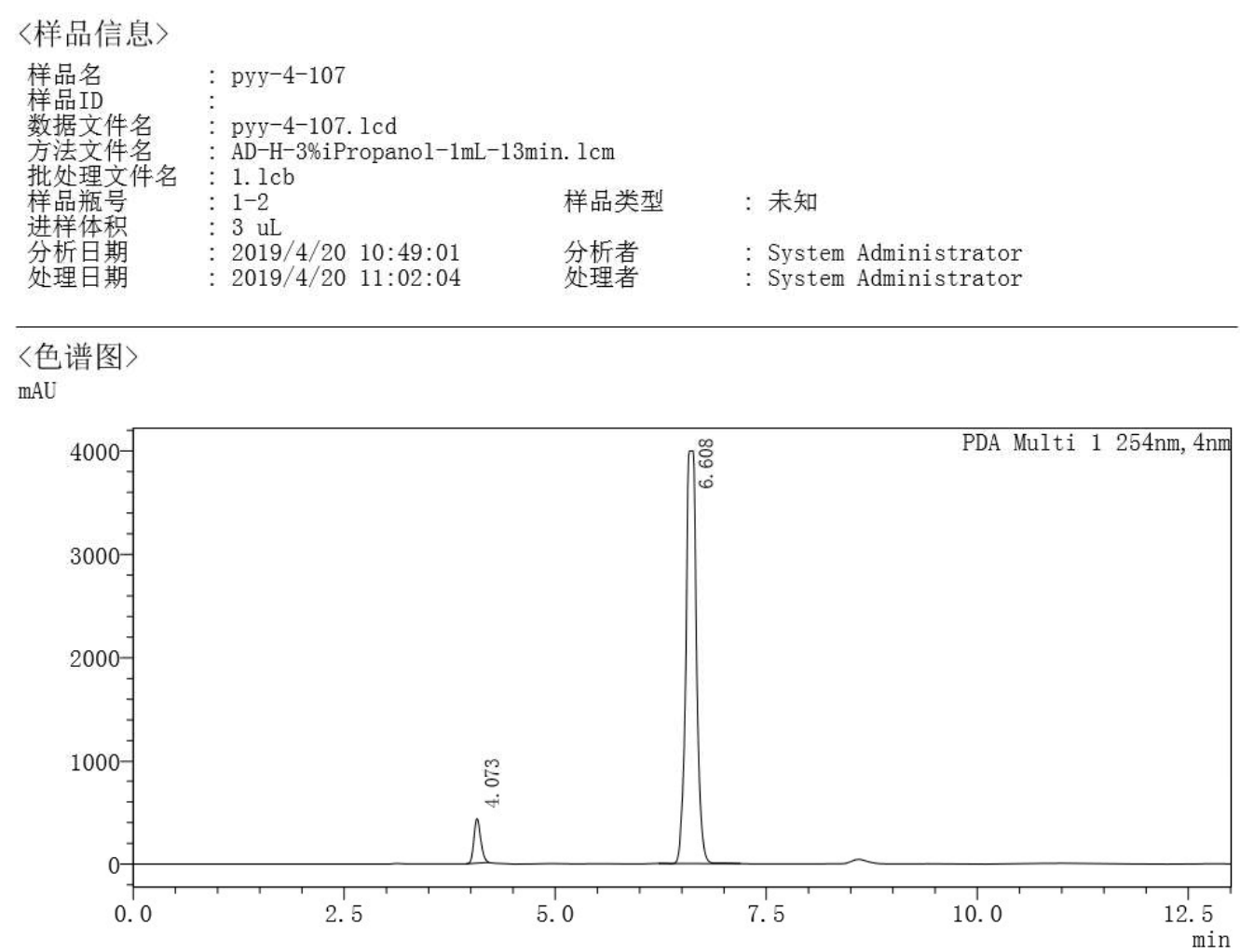

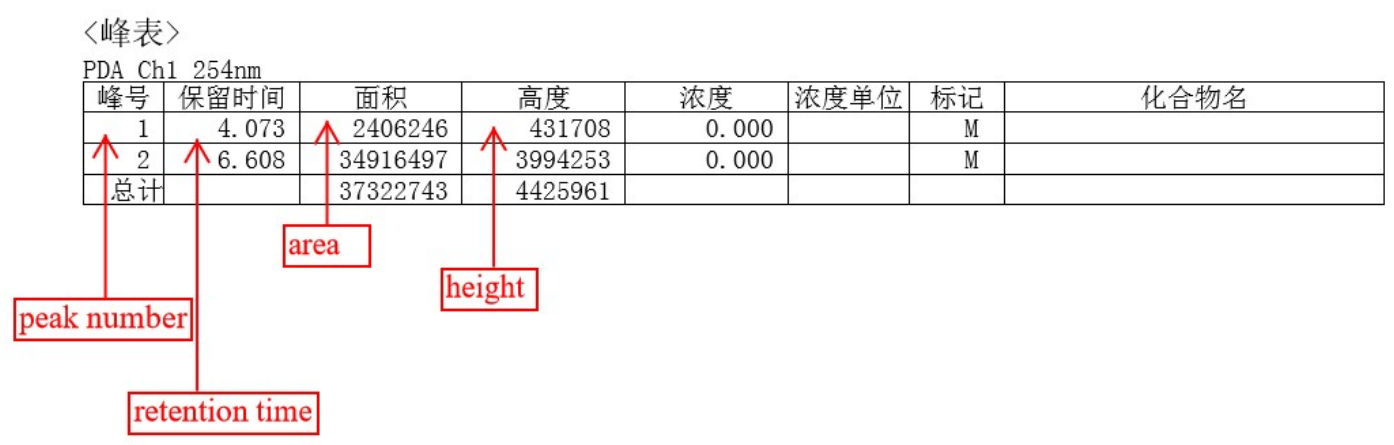


(3aR,8bS)-4,8b-dimethyl-3-(naphthalen-2-yl)-2-pentyl-1,3a,4,8b-

tetrahydrocyclopenta[b]indole (8al)

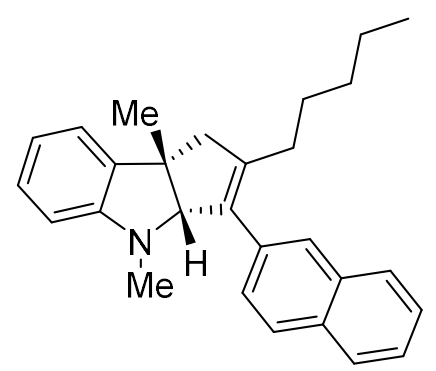

Chemical Formula: $\mathrm{C}_{28} \mathrm{H}_{31} \mathrm{~N}$

Exact Mass: 381.2457

8al was prepared according to general procedure 2.3 using $1 \mathrm{a}$ and $7 \mathrm{I}$ and was purified by silica gel column chromatography (petroleum ether/toluene 2:1-1:99) to obtain 8al as colorless oil (53\% yield). ${ }^{1} \mathrm{H}$ NMR $\left(400 \mathrm{MHz}, \mathrm{CDCl}_{3}\right) \delta$ 7.92-7.78 (m, 3H), 7.75-7.68 (m, 1H), 7.55-7.40 (m, 3H), 7.15-7.04 (m, 2H), $6.68(\mathrm{td}, J=7.4,1.0 \mathrm{~Hz}, 1 \mathrm{H}), 6.33(\mathrm{~d}, J=7.7$ $\mathrm{Hz}, 1 \mathrm{H}), 4.57(\mathrm{~d}, J=1.6 \mathrm{~Hz}, 1 \mathrm{H}), 2.90(\mathrm{~d}, J=16.6 \mathrm{~Hz}, 1 \mathrm{H}), 2.67(\mathrm{~d}, J=16.6 \mathrm{~Hz}, 1 \mathrm{H})$, $2.45(\mathrm{~s}, 3 \mathrm{H}), 2.23-2.06(\mathrm{~m}, 2 \mathrm{H}), 1.56(\mathrm{~s}, 3 \mathrm{H}), 1.47-1.32(\mathrm{~m}, 2 \mathrm{H}), 1.25-1.10(\mathrm{~m}, 4 \mathrm{H}), 0.82$ $(\mathrm{t}, J=6.9 \mathrm{~Hz}, 3 \mathrm{H}) ;{ }^{13} \mathrm{C} \mathrm{NMR}\left(101 \mathrm{MHz}, \mathrm{CDCl}_{3}\right) \delta 151.3,143.0,137.7,135.9,135.8,133.4$, $132.3,127.9,127.8,127.8,127.7,127.3,127.0,126.0,125.6,122.5,116.8,106.3,87.3$, 51.0, 49.6, 35.3, 31.7, 29.0, 28.0, 27.7, 22.5, 14.0; HRMS: (ESI) calcd for $\mathrm{C}_{28} \mathrm{H}_{32} \mathrm{~N}^{+}$ $[\mathrm{M}+\mathrm{H}]^{+}$382.2529; found 382.2532.

The enantiomeric purity was established by HPLC analysis using a chiral column: AD-H column, $30^{\circ} \mathrm{C}, n$-Hexane $/ i-$ Propanol $=98 / 2$ as eluent, $254 \mathrm{~nm}, 0.5 \mathrm{~mL} / \mathrm{min}$. tR $=6 \mathrm{~min}$ (minor), 11 min (major).

Optical Rotation: $[\alpha]_{D^{27}}-93.7$ (c 0.4, ${ }^{\mathrm{PrOH}}$ ) for $91 \%$ ee. 


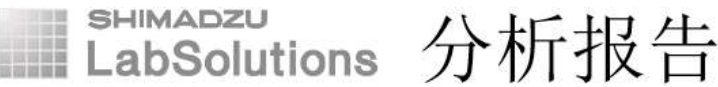

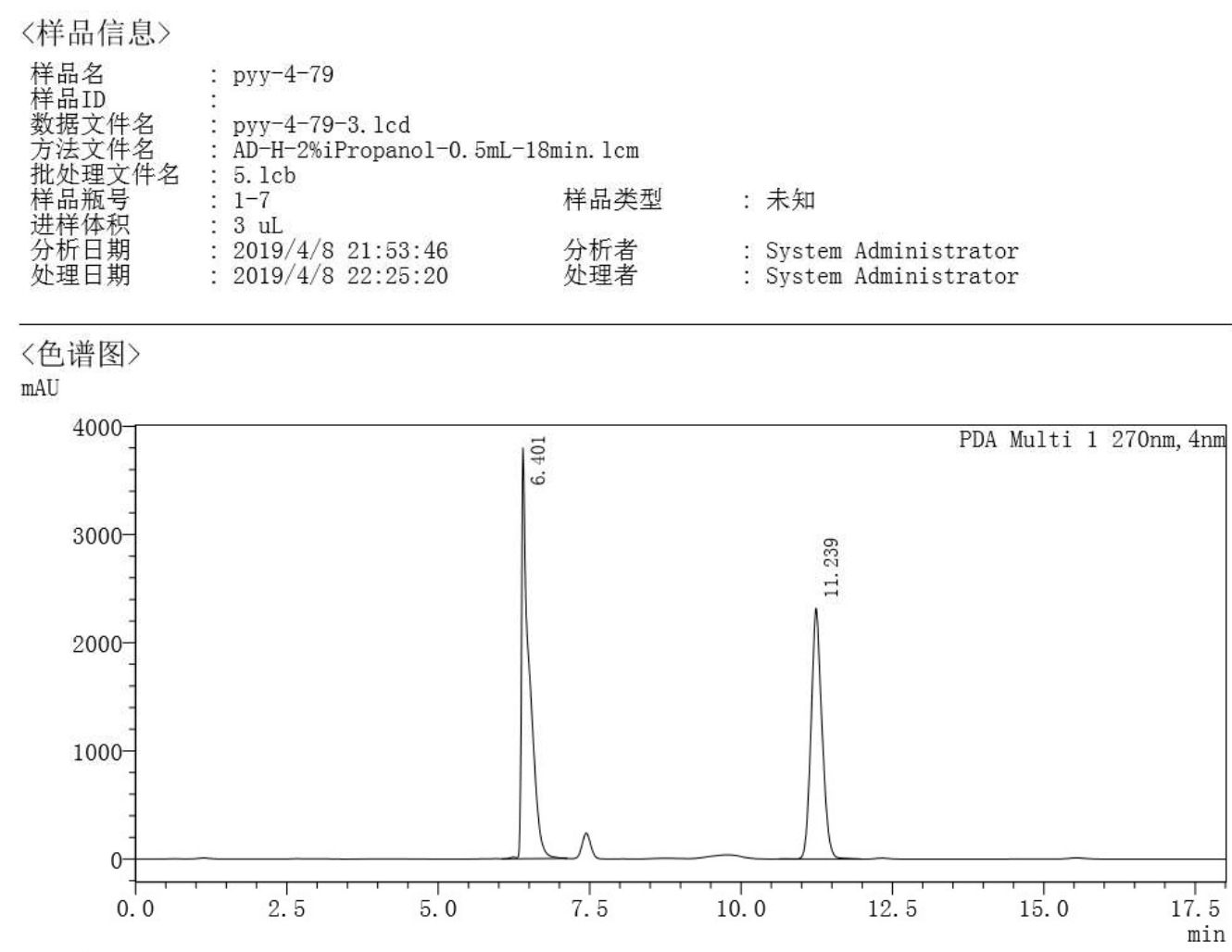

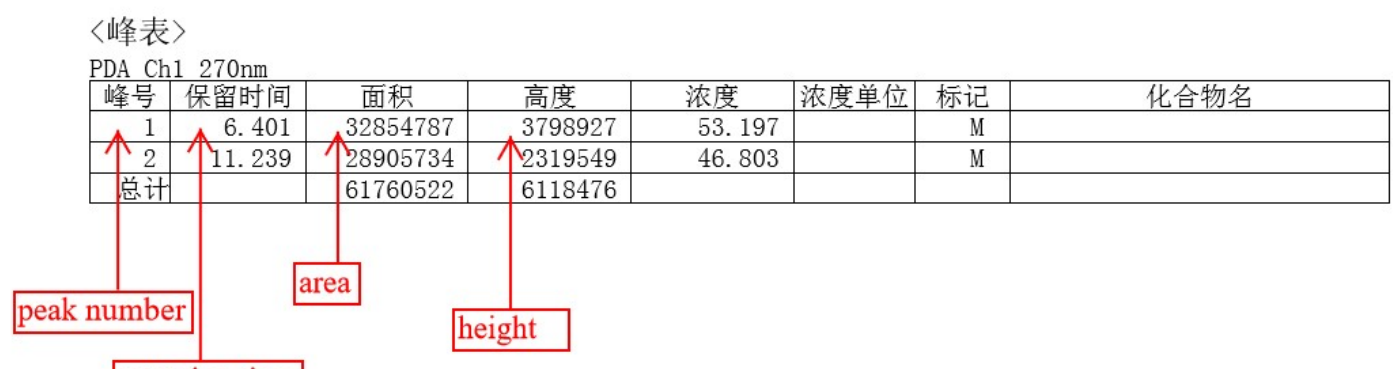

retention time 
In LabSolutions 分析报告

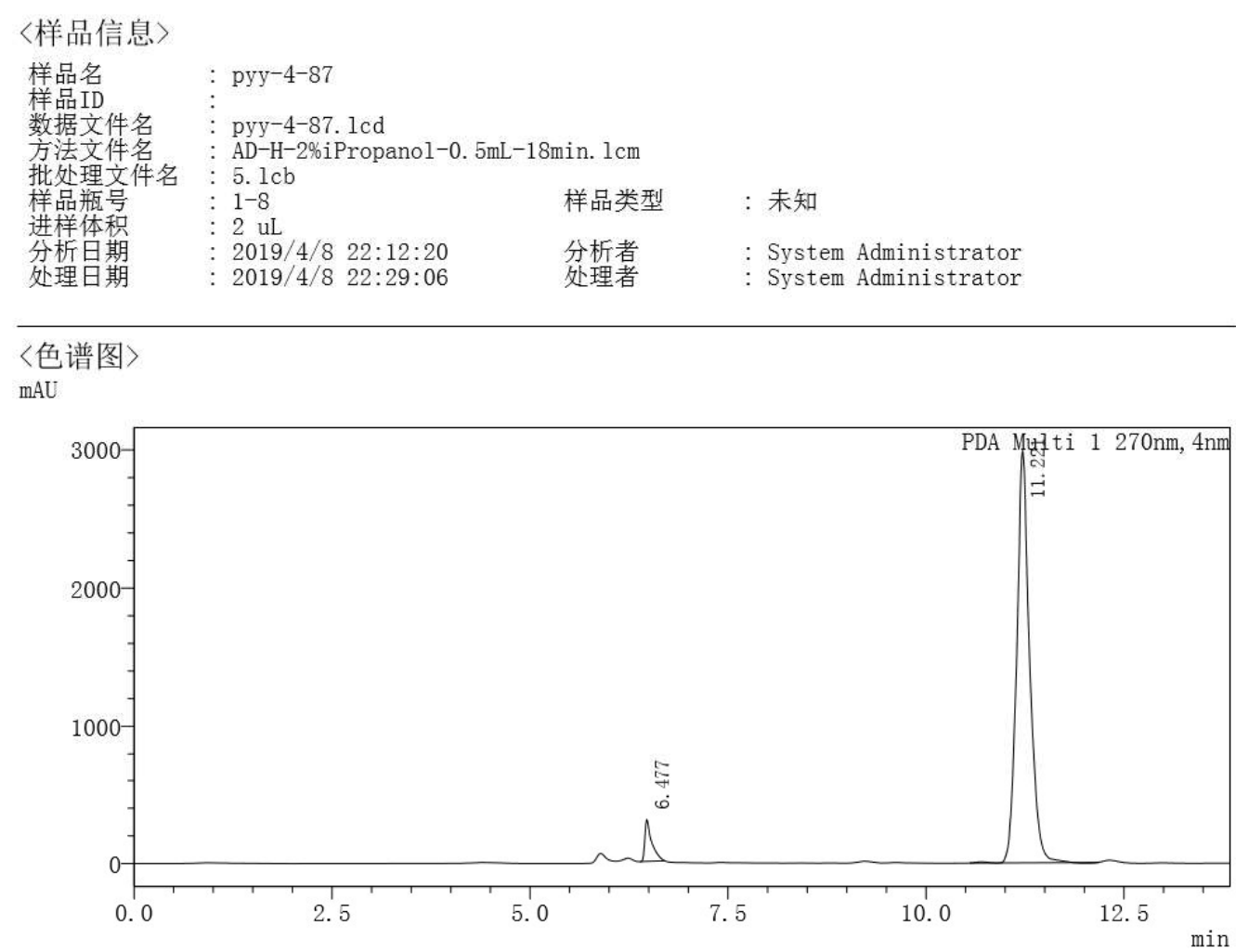

〈峰表〉

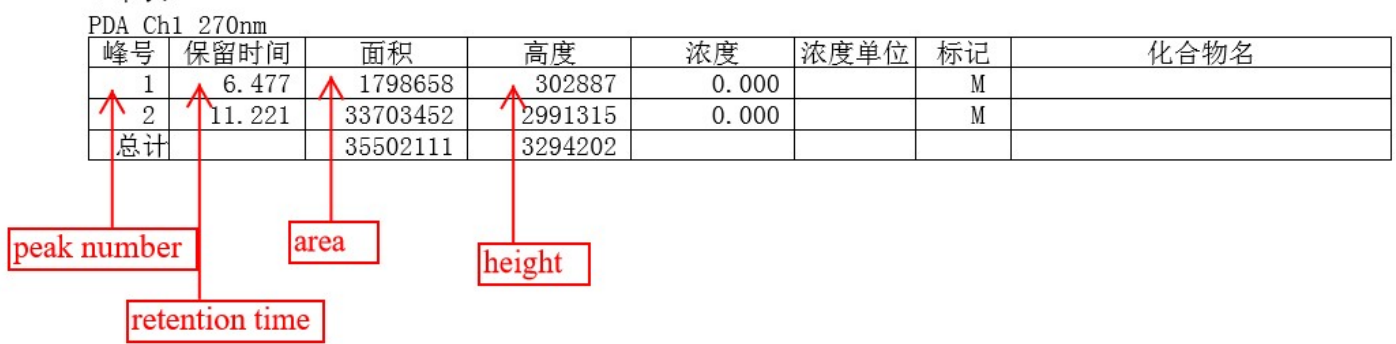


(3aR,8bS)-3-(benzo[b]thiophen-5-yl)-2,4,8b-trimethyl-1,3a,4,8b-

tetrahydrocyclopenta[b]indole (8am)

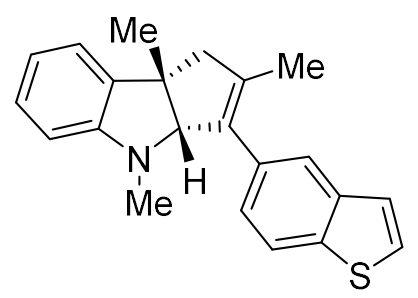

Chemical Formula: $\mathrm{C}_{22} \mathrm{H}_{21} \mathrm{NS}$

Exact Mass: 331.1395

8am was prepared according to general procedure 2.3 using $1 \mathrm{a}$ and $7 \mathrm{~m}$ and was purified by silica gel column chromatography (petroleum ether/toluene 2:1-1:99) to obtain 8am as white solid (56\% yield). ${ }^{1} \mathrm{H}$ NMR $\left(400 \mathrm{MHz}, \mathrm{CDCl}_{3}\right) \delta 7.88(\mathrm{dt}, J=8.4,0.8 \mathrm{~Hz}, 1 \mathrm{H}), 7.74$ (d, $J=1.5 \mathrm{~Hz}, 1 \mathrm{H}), 7.46(\mathrm{~d}, J=5.4 \mathrm{~Hz}, 1 \mathrm{H}), 7.37-7.28(\mathrm{~m}, 2 \mathrm{H}), 7.14-7.06(\mathrm{~m}, 2 \mathrm{H}), 6.68$ (td, $J=7.3,1.0 \mathrm{~Hz}, 1 \mathrm{H}), 6.33(\mathrm{~d}, J=7.6 \mathrm{~Hz}, 1 \mathrm{H}), 4.56(\mathrm{~s}, 1 \mathrm{H}), 2.92(\mathrm{~d}, J=16.7 \mathrm{~Hz}, 1 \mathrm{H})$, $2.63(\mathrm{~d}, J=16.6 \mathrm{~Hz}, 1 \mathrm{H}), 2.49$ (s, 3H), $1.73(\mathrm{~d}, J=1.2 \mathrm{~Hz}, 3 \mathrm{H}), 1.55(\mathrm{~s}, 3 \mathrm{H}) ;{ }^{13} \mathrm{C}$ NMR $\left(101 \mathrm{MHz}, \mathrm{CDCl}_{3}\right) \delta 151.3,139.9,138.2,138.1,137.8,136.0,134.3,127.9,126.7,125.2$, 124.0, 123.6, 122.6, 122.3, 117.0, 106.4 87.3, 54.1, 49.6, 35.4, 28.2, 15.2; HRMS: (ESI) calcd for $\mathrm{C}_{22} \mathrm{H}_{22} \mathrm{NS}^{+}[\mathrm{M}+\mathrm{H}]^{+} 332.1461$; found 332.1468 .

The enantiomeric purity was established by HPLC analysis using a chiral column: AD-H column, $30{ }^{\circ} \mathrm{C}, n$-Hexane $/ i$-Propanol $=98 / 2$ as eluent, $254 \mathrm{~nm}, 0.5 \mathrm{~mL} / \mathrm{min}$. tR $=7 \mathrm{~min}$ (minor), 9 min (major).

Optical Rotation: $[\alpha]_{D^{26}}-0.2$ (c 0.4 , ${ }^{\mathrm{P}} \mathrm{POH}$ ) for $84 \%$ ee. 
1]11 LHMabSolutions 分析报告

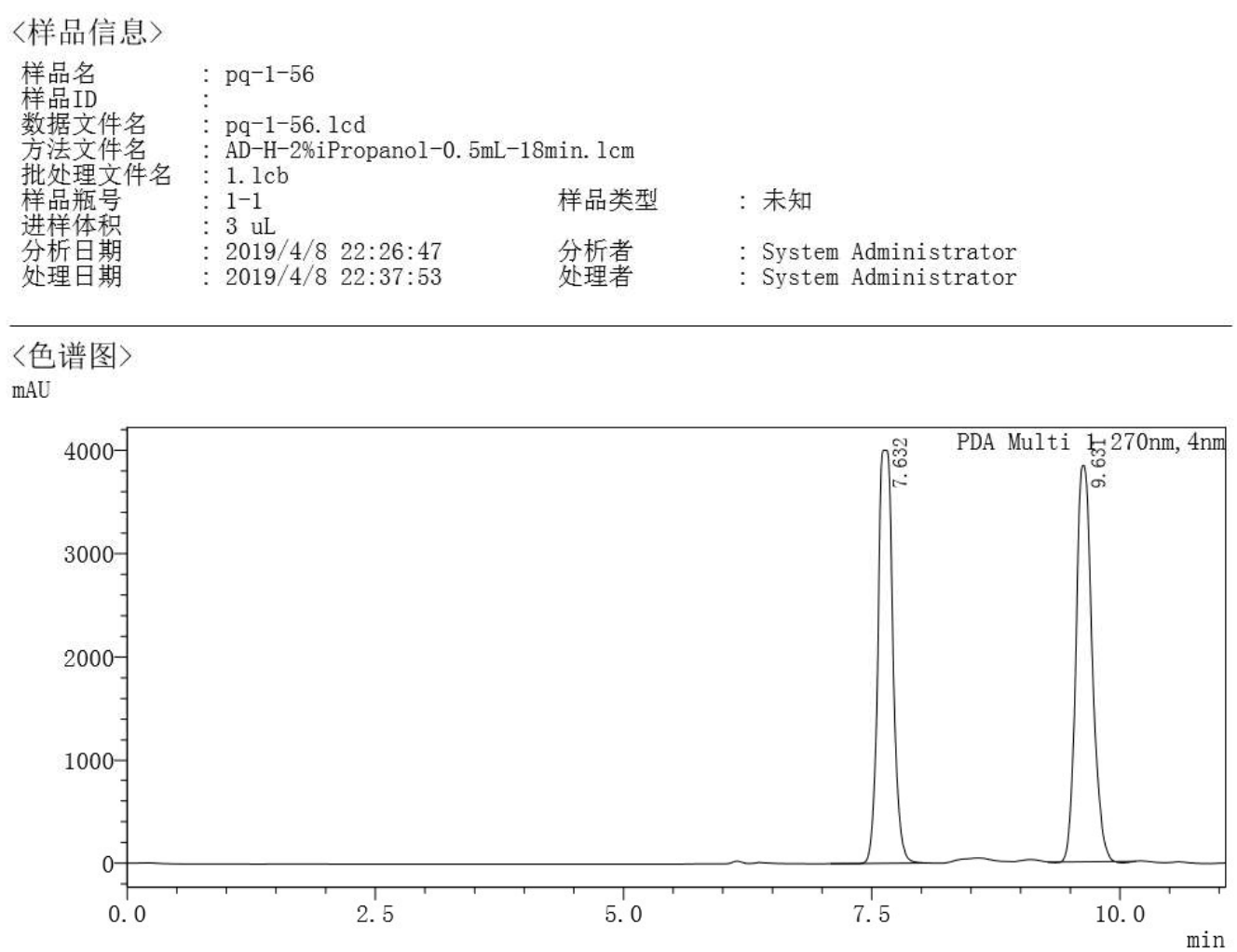

〈峰表〉

\begin{tabular}{|c|c|c|c|c|c|c|c|}
\hline 峰号 & 保留时间 & 面积 & 高度 & 浓度 & 浓度单位 & 标记 & 化合物名 \\
\hline 1 & 7.632 & 41500150 & 4001679 & 0.000 & & M & \\
\hline 仡 & 9.631 & 木 43348483 & 个 3841188 & 0.000 & & M & \\
\hline \begin{tabular}{|l} 
总计 \\
\end{tabular} & & 84848633 & 7842867 & & & & \\
\hline
\end{tabular}

retention time 


\section{LabSolutions 分析报告}

〈样品信息〉
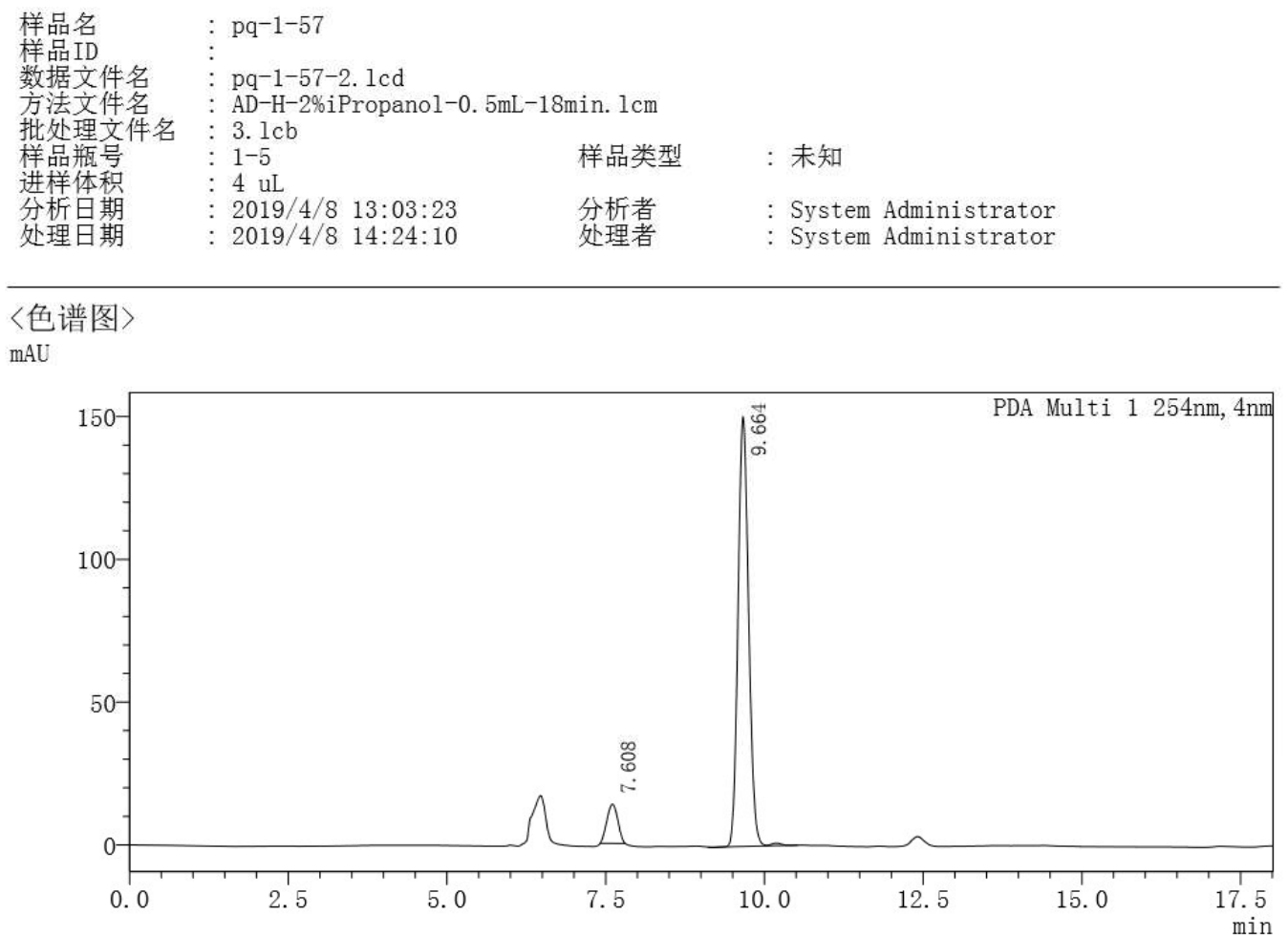

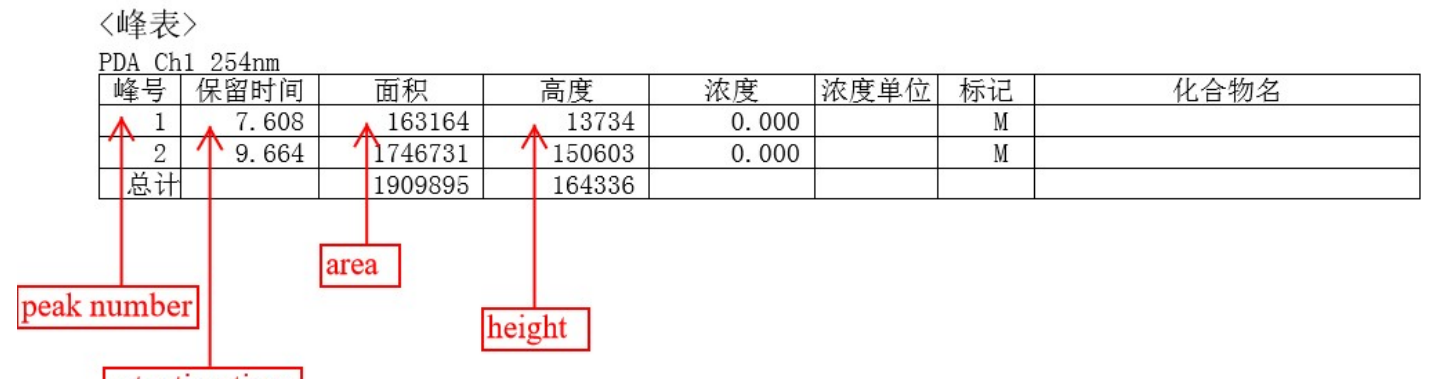

retention time 
(3aR,8bS)-4,8b-dimethyl-2,3-diphenyl-1,3a,4,8b-tetrahydrocyclopenta[b]indole (8an)

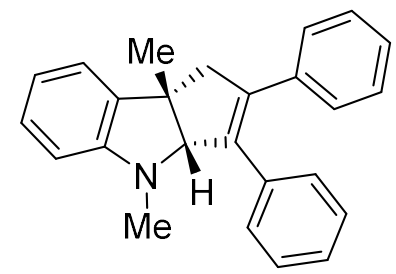

Chemical Formula: $\mathrm{C}_{25} \mathrm{H}_{23} \mathrm{~N}$

Exact Mass: 337.1830

8an was prepared according to general procedure 2.3 using $1 \mathbf{a}$ and $\mathbf{7 n}$ and was purified by silica gel column chromatography (petroleum ether/toluene 2:1-1:99) to obtain 8an as colorless oil (26\% yield). ${ }^{1} \mathrm{H}$ NMR $\left(400 \mathrm{MHz}, \mathrm{CDCl}_{3}\right) \delta$ 7.47-7.26 $(\mathrm{m}, 5 \mathrm{H}), 7.25-7.02(\mathrm{~m}$, $7 \mathrm{H}), 6.75(\mathrm{td}, J=7.4,1.0 \mathrm{~Hz}, 1 \mathrm{H}), 6.43(\mathrm{~d}, J=7.7 \mathrm{~Hz}, 1 \mathrm{H}), 4.59(\mathrm{~d}, J=1.9 \mathrm{~Hz}, 1 \mathrm{H}), 3.45$ (dd, $J=16.4,2.1 \mathrm{~Hz}, 1 \mathrm{H}), 2.92(\mathrm{~d}, J=16.4 \mathrm{~Hz}, 1 \mathrm{H}), 2.55(\mathrm{~s}, 3 \mathrm{H}), 1.63(\mathrm{~s}, 3 \mathrm{H}) ;{ }^{13} \mathrm{C} N M R$ (101 MHz, CDCl $)$ ס 151.6, 139.2, 138.1, 137.7, 137.4, 137.0, 128.9, 128.5, 128.1, 127.9, 127.0, 126.9, 122.5, 117.2, 106.8, 88.6, 52.5, 49.5, 35.9, 27.8; HRMS: (ESI) calcd for $\mathrm{C}_{25} \mathrm{H}_{24} \mathrm{~N}^{+}[\mathrm{M}+\mathrm{H}]^{+} 338.1903$; found 338.1909.

The enantiomeric purity was established by HPLC analysis using a chiral column: OJ-H column, $30{ }^{\circ} \mathrm{C}, n$-Hexane/i-Propanol $=95 / 5$ as eluent, $254 \mathrm{~nm}, 1 \mathrm{~mL} / \mathrm{min}$. tR $=4 \mathrm{~min}$ (major), 8 min (minor).

Optical Rotation: $[\alpha]_{D^{27}}+1.4$ (c 0.4 , ${ }^{i} \mathrm{PrOH}$ ) for $90 \%$ ee. 


\section{LabSolutions 分析报告}

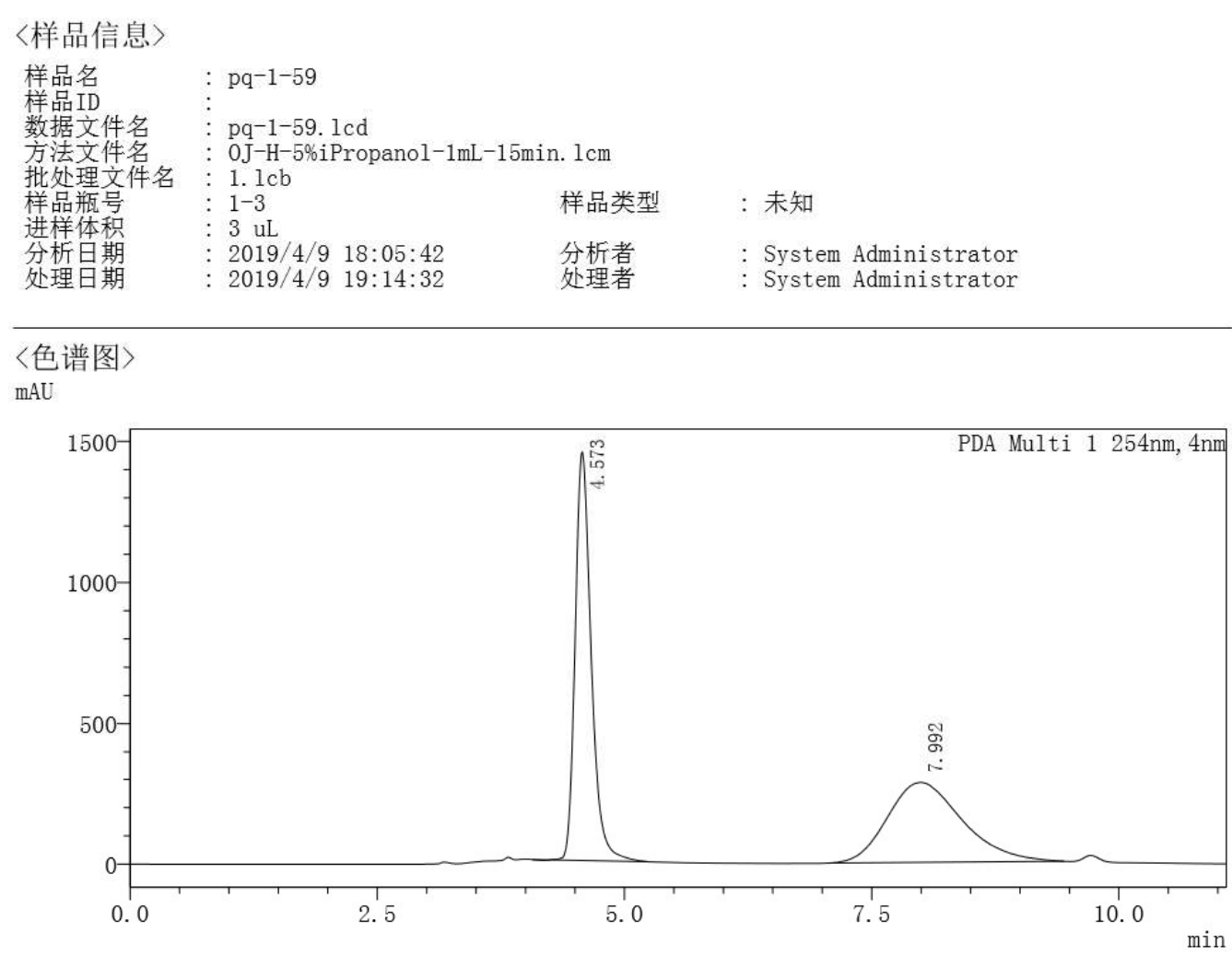

〈峰表〉

\begin{tabular}{|c|c|c|c|c|c|c|c|}
\hline 峰号 & 保留时间 & 面积 & 高度 & 浓度 & 浓度单位 & 标记 & 化合物名 \\
\hline 1 & 4.573 & 16334610 & 1449845 & 0.000 & & M & \\
\hline 木 2 & 7.992 & 木14839134 & 木 282924 & 0.000 & & M & \\
\hline 总计 & & 31173744 & 1732769 & & & & \\
\hline num & & & & & & & \\
\hline
\end{tabular}

retention time 


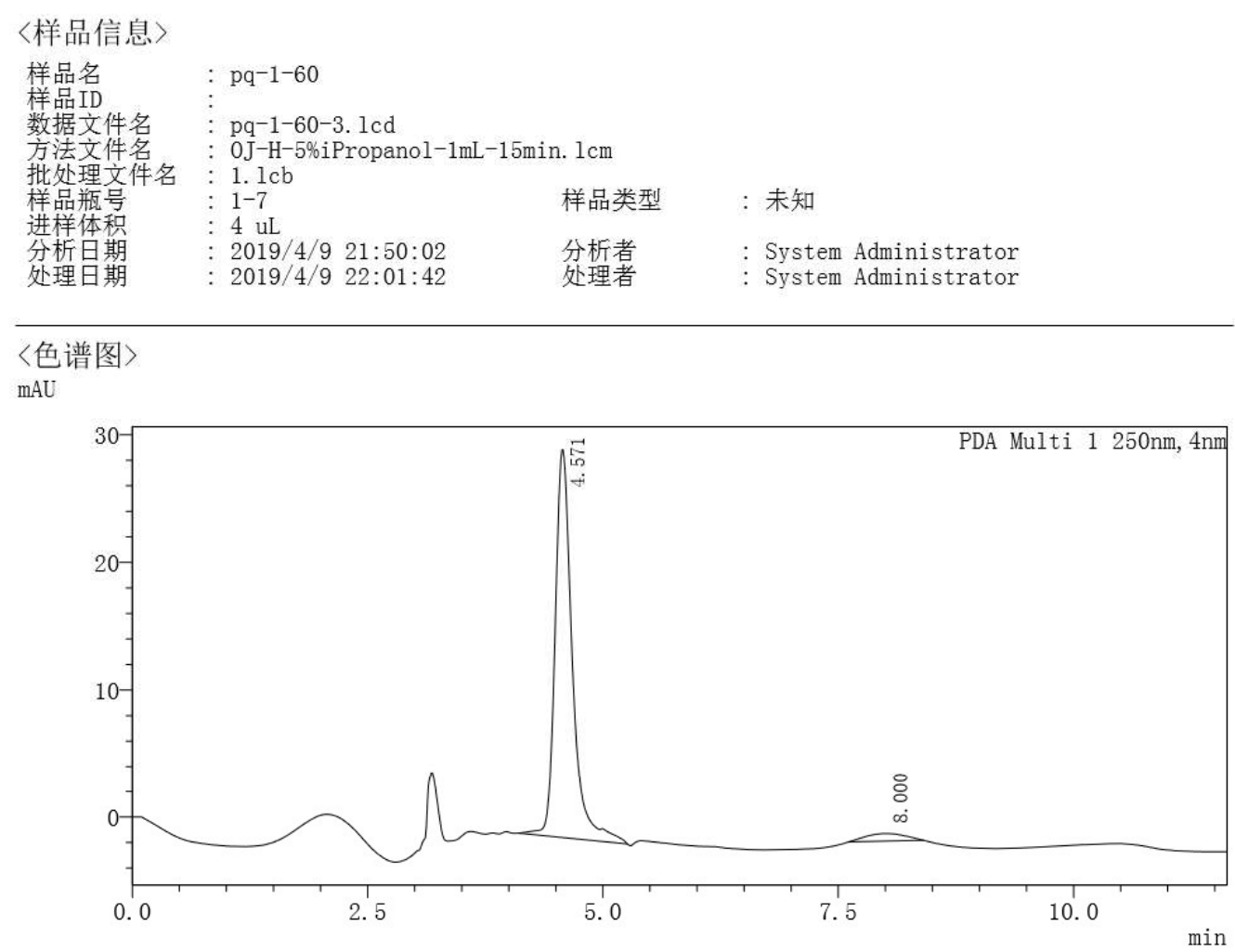

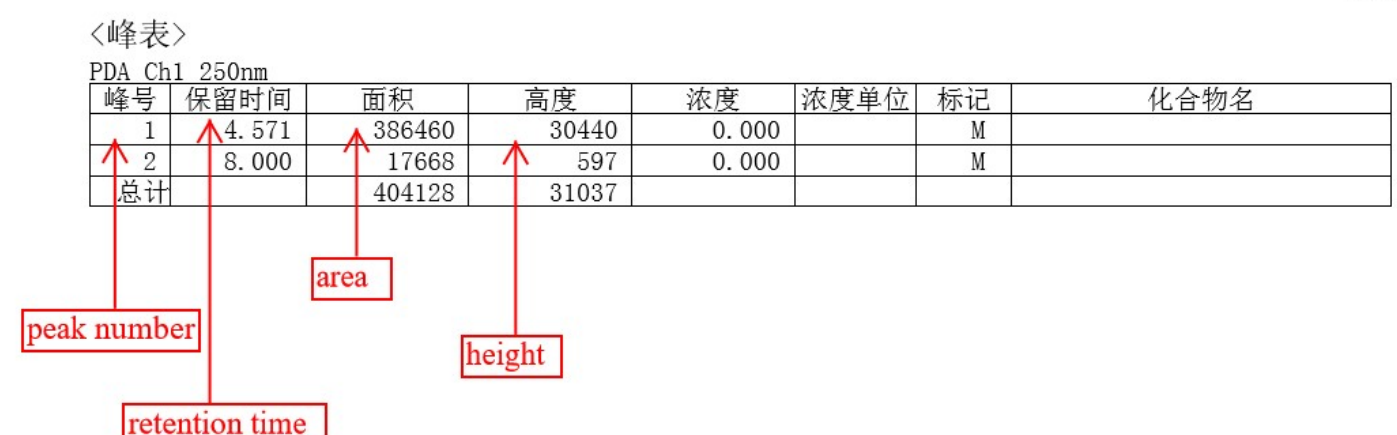


(3aR,8bS)-4,8b-dimethyl-2,3-dipropyl-1,3a,4, 8b-tetrahydrocyclopenta[b]indole (8ao)

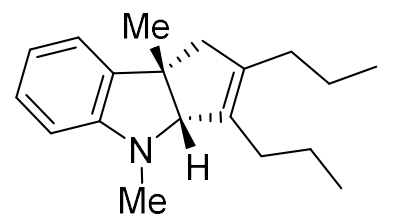

Chemical Formula: $\mathrm{C}_{19} \mathrm{H}_{27} \mathrm{~N}$

Exact Mass: 269.2143

8ao was prepared according to general procedure 2.3 using $1 \mathrm{a}$ and 70 and was purified by silica gel column chromatography (petroleum ether/toluene 2:1-1:99) to obtain 8ao as colorless oil $\left(40 \%\right.$ yield). ${ }^{1} \mathrm{H}$ NMR $\left(400 \mathrm{MHz}, \mathrm{CDCl}_{3}\right) \delta$ 7.11-7.04 $(\mathrm{m}, 1 \mathrm{H}), 7.03-6.99(\mathrm{~m}$, 1H), 6.69-6.61 (m, 1H), 6.43 (d, J = 7.8 Hz, 1H), 3.95 (s, 1H), 2.97 (s, 3H), 2.59 (d, J = $16.2 \mathrm{~Hz}, 1 \mathrm{H}), 2.43(\mathrm{~d}, J=16.1 \mathrm{~Hz}, 1 \mathrm{H}), 2.30-2.17(\mathrm{~m}, 1 \mathrm{H}), 2.16-2.01(\mathrm{~m}, 2 \mathrm{H}), 1.99-1.88$ (m, 1H), 1.69-1.54 (m, 1H), 1.48-1.29 (m, 3H), $1.38(\mathrm{~s}, 3 \mathrm{H}), 0.94(\mathrm{t}, J=7.3 \mathrm{~Hz}, 3 \mathrm{H}), 0.86$ $(\mathrm{t}, J=7.4 \mathrm{~Hz}, 3 \mathrm{H}) ;{ }^{13} \mathrm{C} \mathrm{NMR}\left(101 \mathrm{MHz}, \mathrm{CDCl}_{3}\right) \delta 151.8,139.1,138.4,135.4,127.6,122.5$, $117.3,107.4,85.7,50.3,49.3,37.2,30.5,29.0,28.6,21.24,21.20,14.22,14.17$; HRMS: (ESI) calcd for $\mathrm{C}_{19} \mathrm{H}_{28} \mathrm{~N}^{+}[\mathrm{M}+\mathrm{H}]^{+} 270.2216$; found 270.2219 .

The enantiomeric purity was established by HPLC analysis using a chiral column: OJ-H column, $30^{\circ} \mathrm{C}, n$-Hexane/i-Propanol $=95 / 5$ as eluent, $254 \mathrm{~nm}, 1 \mathrm{~mL} / \mathrm{min}$. $\mathrm{tR}=3 \mathrm{~min}$ (major), 4 min (minor).

Optical Rotation: $[\alpha]_{D^{28}}-4.0$ (c $\left.0.4,{ }^{i} \mathrm{PrOH}\right)$ for $90 \%$ ee. 


\section{Labsolutions 分析报告}

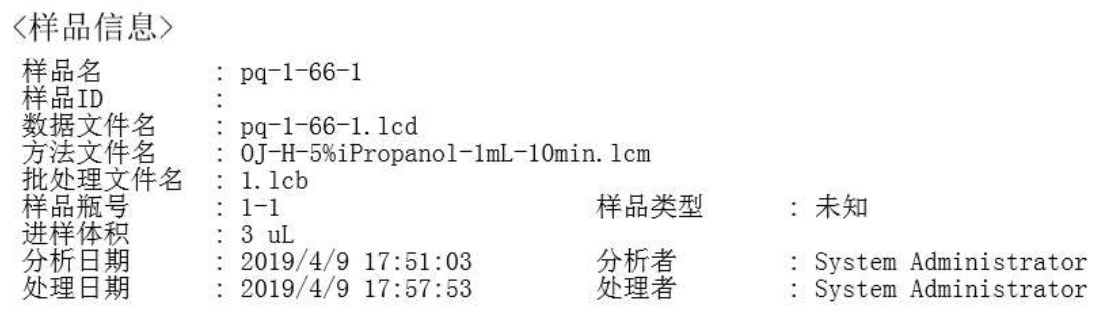

〈色谱图〉

$\mathrm{mAU}$

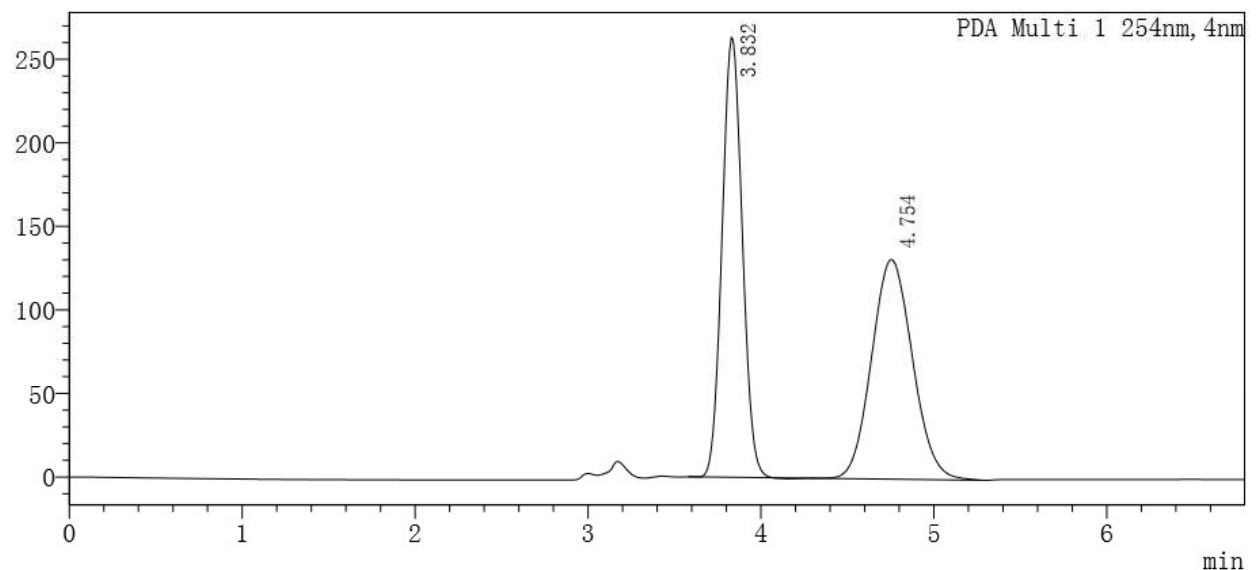

〈峰表〉

\begin{tabular}{|c|c|c|c|c|c|c|c|}
\hline 峰号 & 保留时间 & 面积 & 高度 & 浓度 & 浓度单位 & 标记 & 化合物名 \\
\hline 1 & 3.832 & 2134111 & 263569 & 0.000 & & M & \\
\hline$\uparrow 2$ & 4.754 & 2161224 & 乐 131490 & 0.000 & & M & \\
\hline 总计 & & 4295335 & 395059 & & & & \\
\hline mber & & rea & height & & & & \\
\hline
\end{tabular}

retention time 


\section{LabSolutions 分析报告}

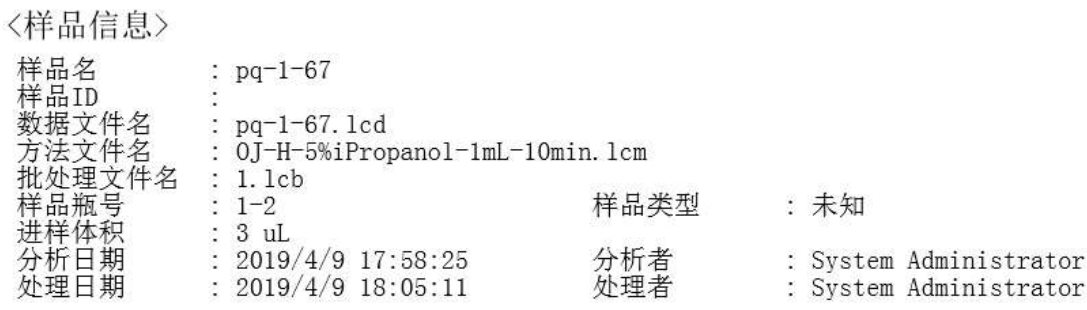

〈色谱图〉

$\mathrm{mAU}$

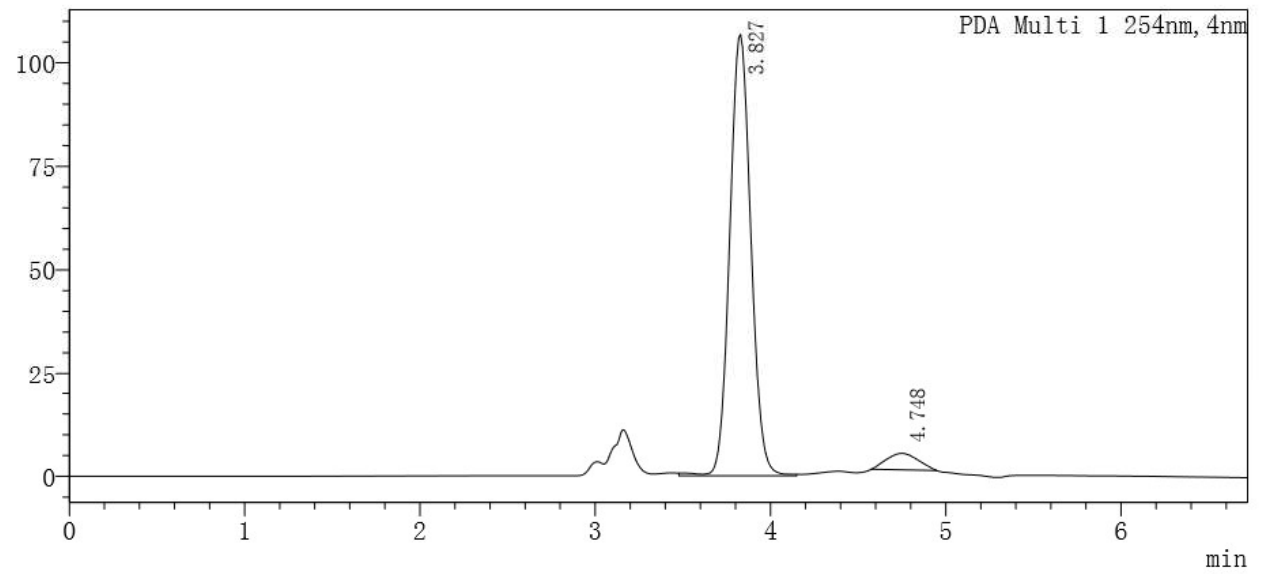

〈峰表〉

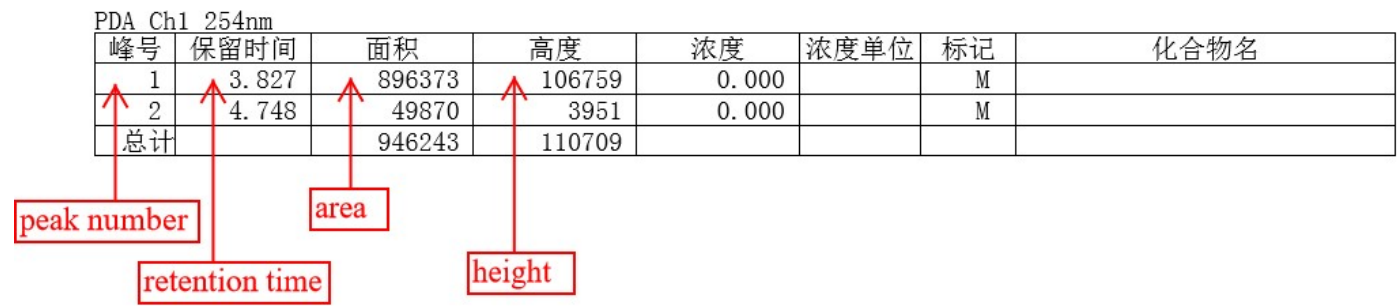




\section{Crystallographic data}

\subsection{Compound 3ka}

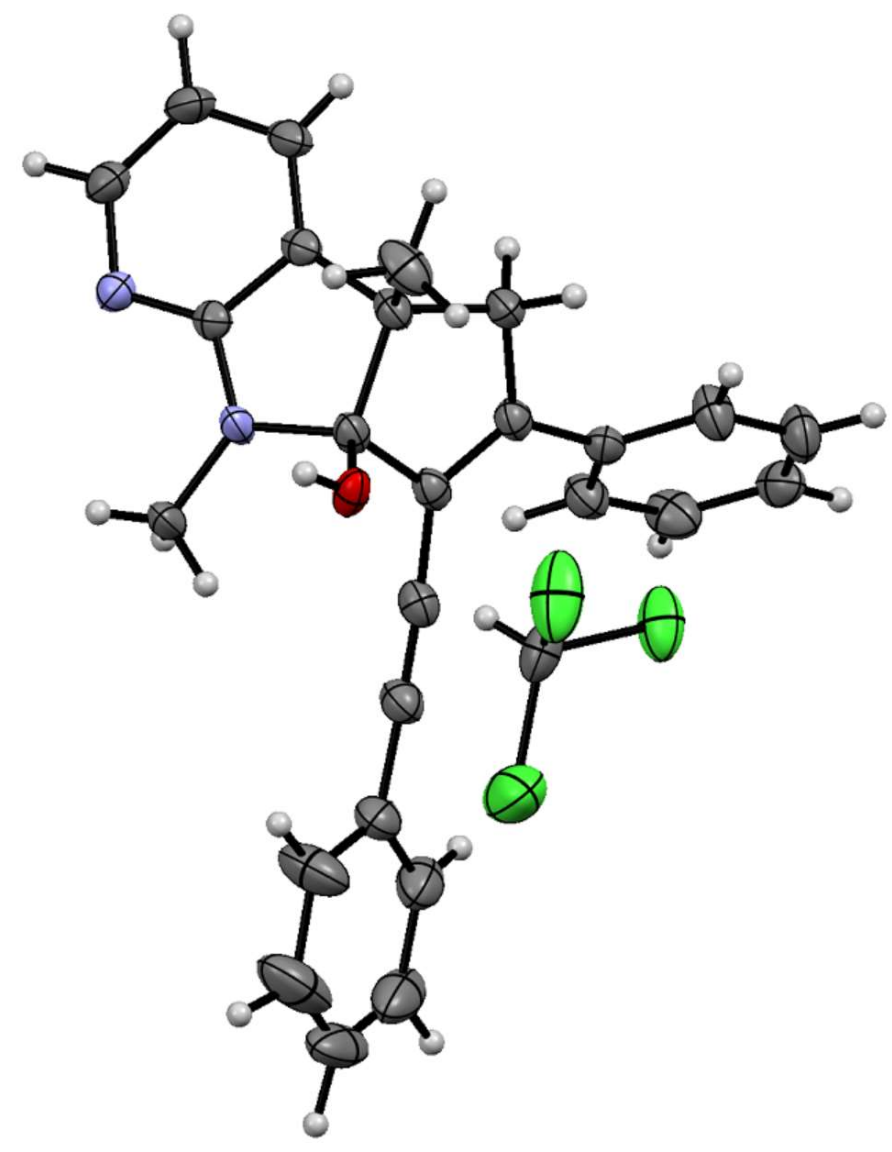

Bond precision:

C-C $=0.0051 \AA$

Wavelength $=1.54184$

Cell:

$$
a=16.9269(5)
$$$$
b=16.7619(5)
$$

alpha $=90$

beta $=91.738(2)$

$$
\text { gamma }=90
$$

Temperature:

$296 \mathrm{~K}$

Calculated

Volume

Space group

Hall group

Moiety formula

Sum formula
2512.18(14)

C c

C - $2 \mathrm{yc}$

$\mathrm{C} 26 \mathrm{H}_{2} 2 \mathrm{~N}_{2} \mathrm{O}, \mathrm{CH} \mathrm{Cl}_{3}$

$\mathrm{C} 27 \mathrm{H} 23 \mathrm{Cl}_{3} \mathrm{~N}_{2} \mathrm{O}$
Reported

2512.18(14)

$\mathrm{C} \mathrm{C}$

C -2yc 


$\begin{array}{lll}\mathrm{Mr} & 497.82 & 1991.29 \\ \mathrm{Dx}, \mathrm{g} \mathrm{cm}-3 & 1.316 & 1.316 \\ \mathrm{Z} & 4 & 1 \\ \mathrm{Mu}(\mathrm{mm}-1) & 3.473 & 3.473 \\ \mathrm{~F} 000 & 1032.0 & 1032.0 \\ \text { F000' } & 1038.68 & \\ \text { h, k, Imax } & 19,19,10 & 19,19,10 \\ \text { Nref } & 4279[2144] & 2977 \\ \text { Tmin, Tmax } & 0.692,0.757 & 0.729,0.788 \\ \text { Tmin' } & 0.510 & \end{array}$

Correction method $=$ \# Reported T Limits: $T \min =0.729 \quad$ Tmax $=0.788$

AbsCorr $=$ MULTI-SCAN

Data completeness $=1.39 / 0.70$

Theta $(\max )=65.043$

$\mathrm{R}$ (reflections) $=0.0315(2960)$

$\mathrm{wR} 2($ reflections $)=0.0813(2977)$

$S=1.071$

Npar $=391$

CCDC 1870707 (3ka) contain the supplementary crystallographic data for this paper. These data can be obtained free of charge from The Cambridge Crystallographic Data Centre. 
7.2 Compound 8aa (racemic)

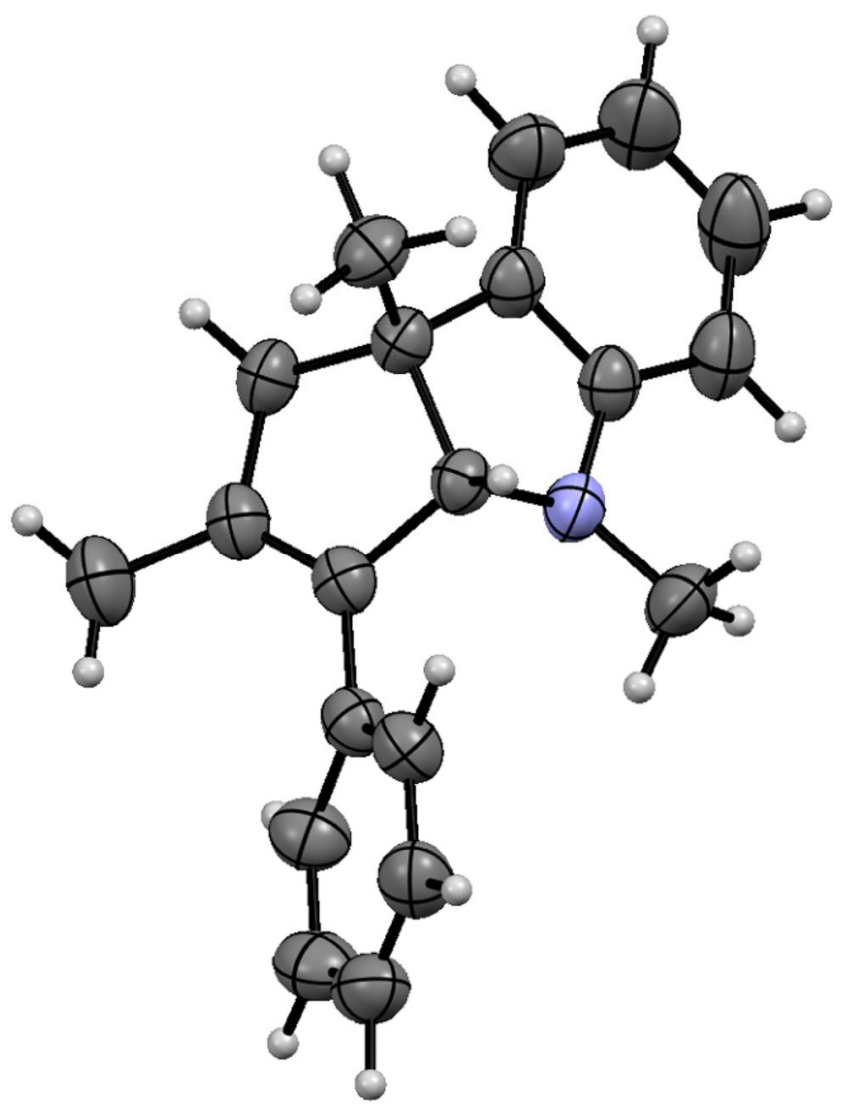

\begin{tabular}{llcr} 
Bond precision: & $\mathrm{C}-\mathrm{C}=0.0017 \AA$ & \multicolumn{2}{c}{ Wavelength=0.71073 } \\
Cell: & $\mathrm{a}=10.3039(5)$ & $\mathrm{b}=11.9306(18)$ & $\mathrm{c}=12.985(2)$ \\
& alpha $=90$ & beta $=90.816(4)$ & Reported \\
Temperature: & $296 \mathrm{~K}$ & $1596.1(4)$ & \\
& Calculated & $\mathrm{P} 21 / \mathrm{c}$ & \\
Volume & $1596.1(4)$ & $-\mathrm{P} 2 \mathrm{ybc}$ & \\
Space group & $\mathrm{P} 21 / \mathrm{C}$ & \\
Hall group & $-\mathrm{P} 2 \mathrm{ybc}$ & $\mathrm{C} 20 \mathrm{H} 21 \mathrm{~N}$ \\
Moiety formula & $\mathrm{C} 20 \mathrm{H} 21 \mathrm{~N}$ & 275.38 \\
Sum formula & $\mathrm{C} 20 \mathrm{H} 21 \mathrm{~N}$ & 1.146
\end{tabular}




$\begin{array}{lll}\text { Z } & 4 & 4 \\ \text { Mu (mm-1) } & 0.066 & 0.066 \\ \text { F000 } & 592.0 & 592.0 \\ \text { F000' } & 592.20 & \\ \text { h, k, Imax } & 13,15,16 & 13,15,16 \\ \text { Nref } & 3694 & 3683 \\ \text { Tmin, Tmax } & 0.992,0.995 & 0.729,0.788 \\ \text { Tmin' } & 0.988 & \end{array}$

Correction method $=$ \# Reported T Limits: Tmin $=0.729 \quad$ Tmax $=0.788$

AbsCorr $=$ MULTI-SCAN

Data completeness $=0.997 \quad$ Theta $(\max )=27.572$

$R($ reflections $)=0.0420(2807) \quad w R 2($ reflections $)=0.1327(3683)$

$S=1.278 \quad$ Npar $=274$

CCDC 1878651 (8aa) contain the supplementary crystallographic data for this paper. These data can be obtained free of charge from The Cambridge Crystallographic Data Centre. 
7.3 Compound $(3 R, 8 S)-8$ aa

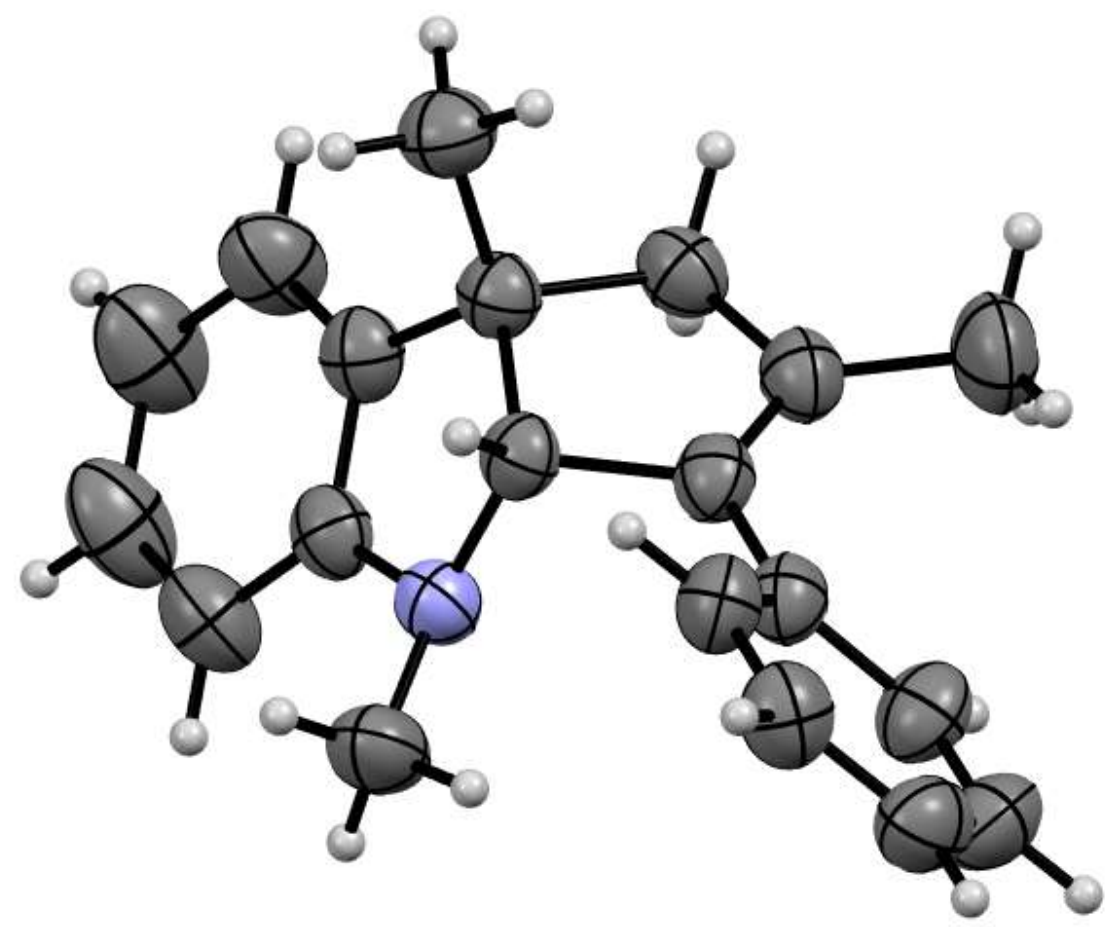

CCDC 1912176 (8aa) contain the supplementary crystallographic data for this paper. These data can be obtained free of charge from The Cambridge Crystallographic Data Centre. 
Table S2. Crystal data and structure refinement for cu_190423B_0m.

Identification code

Empirical formula

Formula weight

Temperature

Wavelength

Crystal system

Space group

Unit cell dimensions

Volume

Z

Density (calculated)

Absorption coefficient

$\mathrm{F}(000)$

Crystal size

Theta range for data collection

Index ranges

Reflections collected

Independent reflections

Completeness to theta $=67.679^{\circ}$

Absorption correction

Max. and min. transmission

Refinement method

Data / restraints / parameters

Goodness-of-fit on $\mathrm{F}^{2}$

Final $R$ indices [l>2sigma(l)]

$\mathrm{R}$ indices (all data)

Extinction coefficient

Largest diff. peak and hole cu_190423b_0m

$\mathrm{C} 20 \mathrm{H} 21 \mathrm{~N}$

275.38

296(2) K

$1.54178 \AA$

Monoclinic

P $121 / \mathrm{c} 1$

$a=10.2763(6) \AA \quad=90^{\circ}$.

$\mathrm{b}=11.8965(6) \AA \quad=90.785(3)^{\circ}$.

$\mathrm{c}=12.9403(7) \AA \quad=90^{\circ}$.

$1581.83(15) \AA^{3}$

4

$1.156 \mathrm{Mg} / \mathrm{m}^{3}$

$0.502 \mathrm{~mm}^{-1}$

592

$0.1 \times 0.1 \times 0.1 \mathrm{~mm}^{3}$

4.303 to $70.255^{\circ}$.

$-11<=\mathrm{h}<=12,-14<=\mathrm{k}<=14,-15<=\mid<=15$

16635

$2814[R$ (int $)=0.0500]$

$96.8 \%$

Semi-empirical from equivalents

0.7533 and 0.6113

Full-matrix least-squares on $\mathrm{F}^{2}$

2814 / 0 / 194

1.124

$\mathrm{R} 1=0.0561, w R 2=0.1374$

$\mathrm{R} 1=0.0604, w R 2=0.1411$

$0.0063(6)$

0.187 and -0.176 e. $\AA^{-3}$ 
Table S3. Atomic coordinates $\left(\times 10^{4}\right)$ and equivalent isotropic displacement parameters $\left(\AA^{2} \times 10^{3}\right)$ for cu_190423B_0m. U(eq) is defined as one third of the trace of the orthogonalized $\mathrm{Uij}^{\mathrm{ij}}$ tensor.

\begin{tabular}{|c|c|c|c|c|}
\hline & $x$ & $y$ & $z$ & $U(e q)$ \\
\hline$C(1)$ & $434(2)$ & $12601(2)$ & $-279(2)$ & $76(1)$ \\
\hline$C(2)$ & $1480(2)$ & $11917(2)$ & $237(2)$ & $51(1)$ \\
\hline$C(3)$ & $1884(2)$ & $12204(2)$ & $1324(2)$ & $53(1)$ \\
\hline$C(4)$ & $3149(2)$ & $11522(2)$ & $1519(1)$ & $45(1)$ \\
\hline$C(5)$ & $3118(2)$ & $10617(2)$ & $659(1)$ & $43(1)$ \\
\hline$C(6)$ & $2142(2)$ & $11042(2)$ & $-138(1)$ & $46(1)$ \\
\hline$C(7)$ & $2003(2)$ & $10511(2)$ & $-1168(2)$ & $48(1)$ \\
\hline$C(8)$ & $3092(2)$ & $10241(2)$ & $-1740(2)$ & $53(1)$ \\
\hline$C(9)$ & $2980(2)$ & $9755(2)$ & $-2710(2)$ & $60(1)$ \\
\hline$C(10)$ & $1761(2)$ & $9532(2)$ & $-3126(2)$ & $63(1)$ \\
\hline$C(11)$ & $677(2)$ & $9787(2)$ & $-2569(2)$ & $66(1)$ \\
\hline$C(12)$ & $785(2)$ & $10262(2)$ & $-1601(2)$ & $60(1)$ \\
\hline$C(13)$ & $4335(2)$ & $12283(2)$ & $1444(2)$ & $58(1)$ \\
\hline$C(14)$ & $3138(2)$ & $10825(2)$ & $2487(2)$ & $48(1)$ \\
\hline$C(15)$ & $2896(2)$ & $9704(2)$ & $2231(2)$ & $48(1)$ \\
\hline$C(16)$ & $2835(2)$ & $8892(2)$ & 2996(2) & $64(1)$ \\
\hline$C(17)$ & $3015(3)$ & $9224(2)$ & $4016(2)$ & $79(1)$ \\
\hline$C(18)$ & $3252(3)$ & $10322(2)$ & $4269(2)$ & $83(1)$ \\
\hline$C(19)$ & $3307(3)$ & 11133(2) & $3503(2)$ & $67(1)$ \\
\hline$C(20)$ & $3052(2)$ & $8519(2)$ & $694(2)$ & $65(1)$ \\
\hline $\mathrm{N}(1)$ & $2710(2)$ & $9569(1)$ & $1172(1)$ & $49(1)$ \\
\hline
\end{tabular}


Table S4. Bond lengths $[\AA]$ and angles $\left[^{\circ}\right]$ for cu_190423B_0m.

\begin{tabular}{|c|c|}
\hline$C(1)-C(2)$ & $1.498(3)$ \\
\hline$C(2)-C(3)$ & $1.501(3)$ \\
\hline$C(2)-C(6)$ & $1.338(3)$ \\
\hline$C(3)-C(4)$ & $1.550(3)$ \\
\hline$C(4)-C(5)$ & $1.549(3)$ \\
\hline$C(4)-C(13)$ & $1.522(3)$ \\
\hline$C(4)-C(14)$ & $1.502(3)$ \\
\hline$C(5)-C(6)$ & $1.515(2)$ \\
\hline$C(5)-N(1)$ & $1.477(2)$ \\
\hline$C(6)-C(7)$ & $1.479(3)$ \\
\hline$C(7)-C(8)$ & $1.389(3)$ \\
\hline$C(7)-C(12)$ & $1.396(3)$ \\
\hline $\mathrm{C}(8)-\mathrm{C}(9)$ & $1.384(3)$ \\
\hline$C(9)-C(10)$ & $1.382(3)$ \\
\hline$C(10)-C(11)$ & $1.369(3)$ \\
\hline$C(11)-C(12)$ & $1.378(3)$ \\
\hline$C(14)-C(15)$ & $1.396(3)$ \\
\hline$C(14)-C(19)$ & $1.374(3)$ \\
\hline$C(15)-C(16)$ & $1.385(3)$ \\
\hline$C(15)-N(1)$ & $1.390(3)$ \\
\hline$C(16)-C(17)$ & $1.388(3)$ \\
\hline$C(17)-C(18)$ & $1.368(4)$ \\
\hline$C(18)-C(19)$ & $1.384(3)$ \\
\hline$C(20)-N(1)$ & $1.440(3)$ \\
\hline$C(1)-C(2)-C(3)$ & $118.82(19)$ \\
\hline$C(6)-C(2)-C(1)$ & $128.8(2)$ \\
\hline$C(6)-C(2)-C(3)$ & $112.43(17)$ \\
\hline$C(2)-C(3)-C(4)$ & $104.72(15)$ \\
\hline$C(5)-C(4)-C(3)$ & $103.76(15)$ \\
\hline$C(13)-C(4)-C(3)$ & $110.39(17)$ \\
\hline$C(13)-C(4)-C(5)$ & $112.12(15)$ \\
\hline$C(14)-C(4)-C(3)$ & $114.12(15)$ \\
\hline$C(14)-C(4)-C(5)$ & $102.46(15)$ \\
\hline
\end{tabular}




$\begin{array}{ll}\mathrm{C}(14)-\mathrm{C}(4)-\mathrm{C}(13) & 113.35(16) \\ \mathrm{C}(6)-\mathrm{C}(5)-\mathrm{C}(4) & 105.33(14) \\ \mathrm{N}(1)-\mathrm{C}(5)-\mathrm{C}(4) & 105.46(14) \\ \mathrm{N}(1)-\mathrm{C}(5)-\mathrm{C}(6) & 113.48(15) \\ \mathrm{C}(2)-\mathrm{C}(6)-\mathrm{C}(5) & 110.35(17) \\ \mathrm{C}(2)-\mathrm{C}(6)-\mathrm{C}(7) & 127.98(17) \\ \mathrm{C}(7)-\mathrm{C}(6)-\mathrm{C}(5) & 121.68(16) \\ \mathrm{C}(8)-\mathrm{C}(7)-\mathrm{C}(6) & 120.75(17) \\ \mathrm{C}(8)-\mathrm{C}(7)-\mathrm{C}(12) & 117.43(19) \\ \mathrm{C}(12)-\mathrm{C}(7)-\mathrm{C}(6) & 121.82(18) \\ \mathrm{C}(9)-\mathrm{C}(8)-\mathrm{C}(7) & 121.50(19) \\ \mathrm{C}(10)-\mathrm{C}(9)-\mathrm{C}(8) & 119.8(2) \\ \mathrm{C}(11)-\mathrm{C}(10)-\mathrm{C}(9) & 119.5(2) \\ \mathrm{C}(10)-\mathrm{C}(11)-\mathrm{C}(12) & 120.9(2) \\ \mathrm{C}(11)-\mathrm{C}(12)-\mathrm{C}(7) & 120.9(2) \\ \mathrm{C}(15)-\mathrm{C}(14)-\mathrm{C}(4) & 109.43(16) \\ \mathrm{C}(19)-\mathrm{C}(14)-\mathrm{C}(4) & 130.48(19) \\ \mathrm{C}(19)-\mathrm{C}(14)-\mathrm{C}(15) & 120.08(19) \\ \mathrm{C}(16)-\mathrm{C}(15)-\mathrm{C}(14) & 120.44(19) \\ \mathrm{C}(16)-\mathrm{C}(15)-\mathrm{N}(1) & 128.05(19) \\ \mathrm{N}(1)-\mathrm{C}(15)-\mathrm{C}(14) & 111.49(16) \\ \mathrm{C}(15)-\mathrm{C}(16)-\mathrm{C}(17) & 118.3(2) \\ \mathrm{C}(18)-\mathrm{C}(17)-\mathrm{C}(16) & 121.3(2) \\ \mathrm{C}(17)-\mathrm{C}(18)-\mathrm{C}(19) & 120.2(2) \\ \mathrm{C}(14)-\mathrm{C}(19)-\mathrm{C}(18) & 119.6(2) \\ \mathrm{C}(15)-\mathrm{N}(1)-\mathrm{C}(5) & 108.04(15) \\ \mathrm{C}(15)-\mathrm{N}(1)-\mathrm{C}(20) & 119.59(17) \\ \mathrm{C}(20)-\mathrm{N}(1)-\mathrm{C}(5) & 117.80(16) \\ & \end{array}$

Symmetry transformations used to generate equivalent atoms: 
Table S5. Anisotropic displacement parameters $\left(\AA^{2} \times 10^{3}\right)$ for cu_190423B_0m. The anisotropic displacement factor exponent takes the form: $-2 \quad{ }^{2}\left[h^{2} a^{* 2} U^{11}+\ldots+2 h k a^{*} b^{*} U^{12}\right]$

\begin{tabular}{|c|c|c|c|c|c|c|}
\hline & $U^{11}$ & $U^{22}$ & $U^{33}$ & $U^{23}$ & $U^{13}$ & $U^{12}$ \\
\hline$C(1)$ & $64(2)$ & $78(2)$ & $85(2)$ & $7(1)$ & $-11(1)$ & $23(1)$ \\
\hline$C(2)$ & $45(1)$ & $52(1)$ & $56(1)$ & $7(1)$ & $0(1)$ & $4(1)$ \\
\hline$C(3)$ & $53(1)$ & $46(1)$ & $58(1)$ & $-1(1)$ & $4(1)$ & $8(1)$ \\
\hline$C(4)$ & $43(1)$ & $46(1)$ & $47(1)$ & $-1(1)$ & $2(1)$ & $2(1)$ \\
\hline$C(5)$ & $37(1)$ & $48(1)$ & $46(1)$ & $-1(1)$ & $2(1)$ & $3(1)$ \\
\hline$C(6)$ & $39(1)$ & $51(1)$ & $48(1)$ & $5(1)$ & $-1(1)$ & $-1(1)$ \\
\hline$C(7)$ & $44(1)$ & $52(1)$ & $47(1)$ & $7(1)$ & $-1(1)$ & $0(1)$ \\
\hline$C(8)$ & $48(1)$ & $61(1)$ & $51(1)$ & $3(1)$ & $-4(1)$ & $2(1)$ \\
\hline$C(9)$ & $62(1)$ & $66(1)$ & $51(1)$ & $2(1)$ & $5(1)$ & $8(1)$ \\
\hline$C(10)$ & $75(2)$ & $62(1)$ & $52(1)$ & $-2(1)$ & $-7(1)$ & $-5(1)$ \\
\hline$C(11)$ & $60(1)$ & $76(2)$ & $61(1)$ & $-2(1)$ & $-10(1)$ & $-14(1)$ \\
\hline$C(12)$ & $45(1)$ & $72(1)$ & $61(1)$ & $1(1)$ & $1(1)$ & $-6(1)$ \\
\hline$C(13)$ & $56(1)$ & $55(1)$ & $64(1)$ & $-2(1)$ & $0(1)$ & $-7(1)$ \\
\hline$C(14)$ & $47(1)$ & $53(1)$ & $45(1)$ & $0(1)$ & $4(1)$ & $8(1)$ \\
\hline$C(15)$ & $42(1)$ & $50(1)$ & $51(1)$ & $4(1)$ & $5(1)$ & $7(1)$ \\
\hline$C(16)$ & $74(2)$ & $54(1)$ & $63(1)$ & $9(1)$ & $11(1)$ & $12(1)$ \\
\hline$C(17)$ & $111(2)$ & $73(2)$ & $54(1)$ & $18(1)$ & $20(1)$ & $23(1)$ \\
\hline$C(18)$ & $122(2)$ & $82(2)$ & $44(1)$ & $2(1)$ & $11(1)$ & $20(2)$ \\
\hline$C(19)$ & $89(2)$ & $60(1)$ & $52(1)$ & $-6(1)$ & $5(1)$ & $10(1)$ \\
\hline$C(20)$ & $80(2)$ & $48(1)$ & $68(1)$ & $-7(1)$ & $-4(1)$ & $0(1)$ \\
\hline $\mathrm{N}(1)$ & $53(1)$ & $44(1)$ & $51(1)$ & $-1(1)$ & $-2(1)$ & $3(1)$ \\
\hline
\end{tabular}


Table S6. Hydrogen coordinates $\left(x 10^{4}\right)$ and isotropic displacement parameters $\left(\AA^{2} \times 10^{3}\right)$ for cu_190423B_0m.

\begin{tabular}{|c|c|c|c|c|}
\hline & $x$ & $y$ & $z$ & $\mathrm{U}(\mathrm{eq})$ \\
\hline$H(1 A)$ & -389 & 12435 & 29 & 114 \\
\hline $\mathrm{H}(1 \mathrm{~B})$ & 395 & 12421 & -1002 & 114 \\
\hline$H(1 C)$ & 625 & 13386 & -195 & 114 \\
\hline $\mathrm{H}(3 \mathrm{~A})$ & 1216 & 11988 & 1808 & 63 \\
\hline $\mathrm{H}(3 \mathrm{~B})$ & 2047 & 13004 & 1393 & 63 \\
\hline $\mathrm{H}(5)$ & 3979 & 10529 & 353 & 52 \\
\hline $\mathrm{H}(8)$ & 3916 & 10389 & -1467 & 64 \\
\hline $\mathrm{H}(9)$ & 3722 & 9580 & -3080 & 72 \\
\hline $\mathrm{H}(10)$ & 1679 & 9211 & -3778 & 76 \\
\hline $\mathrm{H}(11)$ & -143 & 9638 & -2849 & 79 \\
\hline $\mathrm{H}(12)$ & 37 & 10418 & -1231 & 71 \\
\hline$H(13 A)$ & 4332 & 12648 & 782 & 87 \\
\hline$H(13 B)$ & 5112 & 11842 & 1523 & 87 \\
\hline$H(13 C)$ & 4308 & 12840 & 1981 & 87 \\
\hline$H(16)$ & 2677 & 8144 & 2830 & 76 \\
\hline $\mathrm{H}(17)$ & 2975 & 8689 & 4538 & 95 \\
\hline $\mathrm{H}(18)$ & 3376 & 10525 & 4957 & 99 \\
\hline $\mathrm{H}(19)$ & 3457 & 11881 & 3676 & 80 \\
\hline $\mathrm{H}(20 \mathrm{~A})$ & 2796 & 8535 & -22 & 97 \\
\hline $\mathrm{H}(20 \mathrm{~B})$ & 2612 & 7914 & 1034 & 97 \\
\hline $\mathrm{H}(20 \mathrm{C})$ & 3975 & 8408 & 750 & 97 \\
\hline
\end{tabular}

Table S7. Torsion angles [ $\left.{ }^{\circ}\right]$ for cu_190423B_0m. 


\section{Copies of the ${ }^{1} \mathrm{H},{ }^{13} \mathrm{C}$ and ${ }^{19} \mathrm{~F}$ NMR spectra}

\section{3aa}

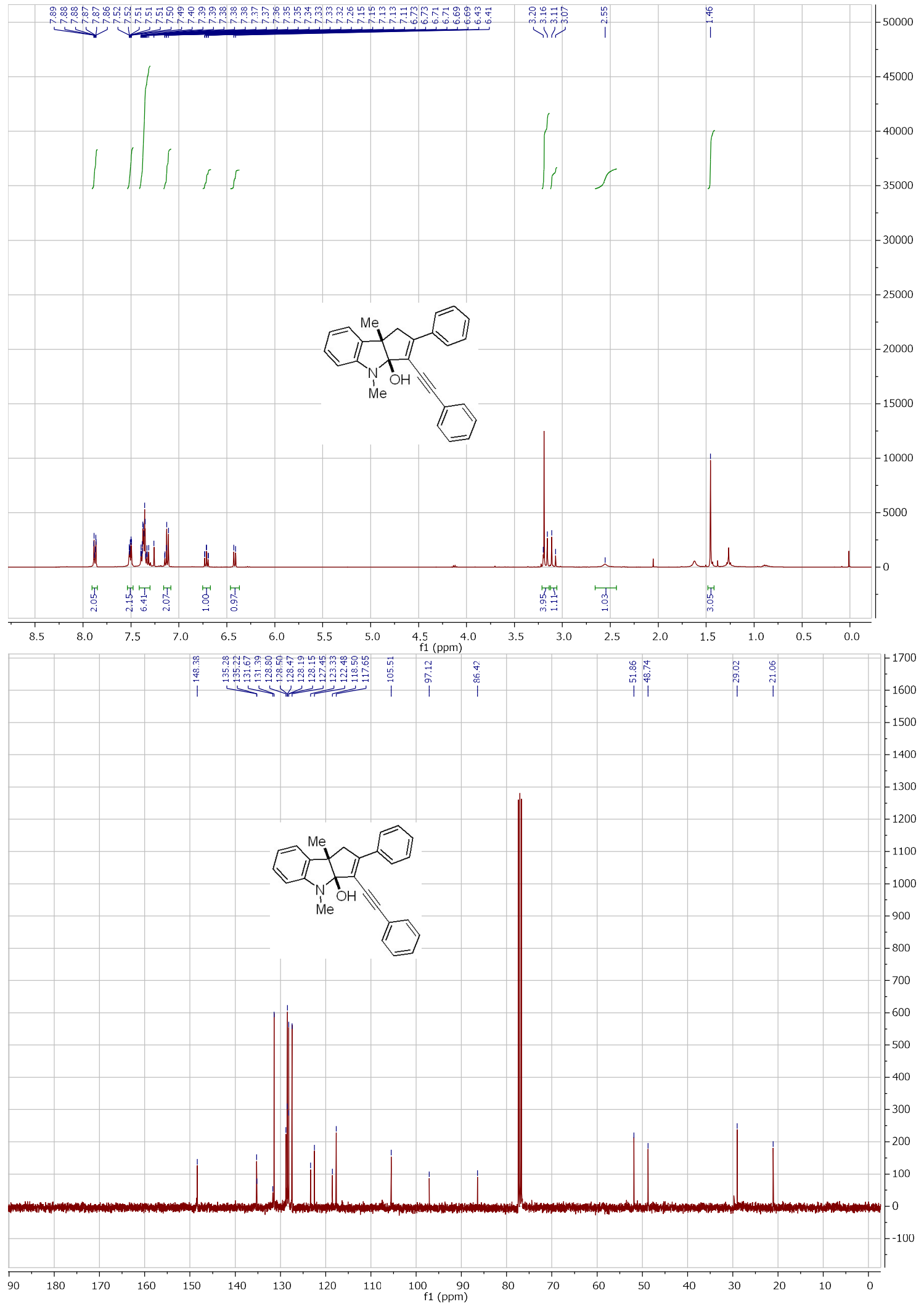




\section{$3 b a$}

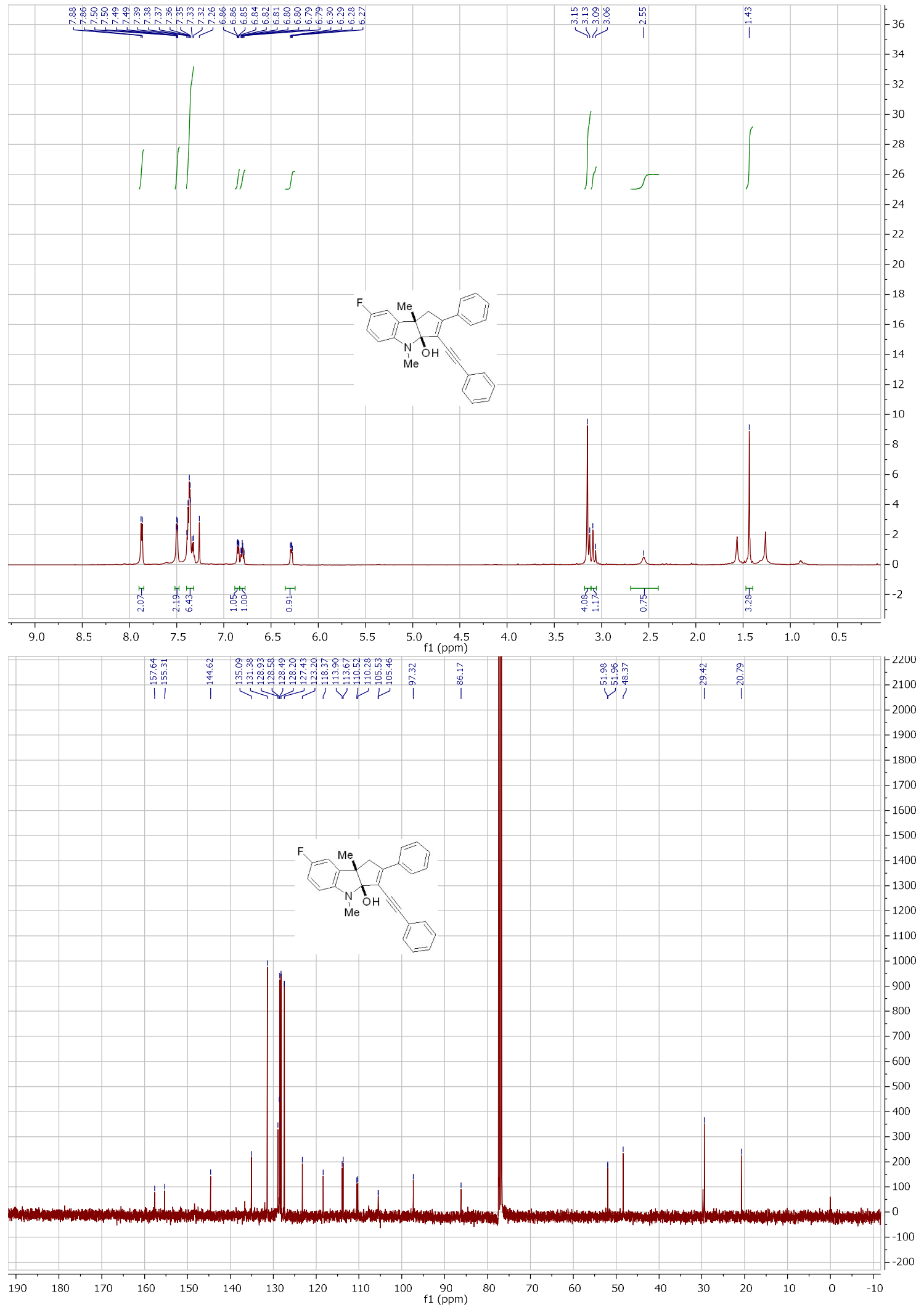




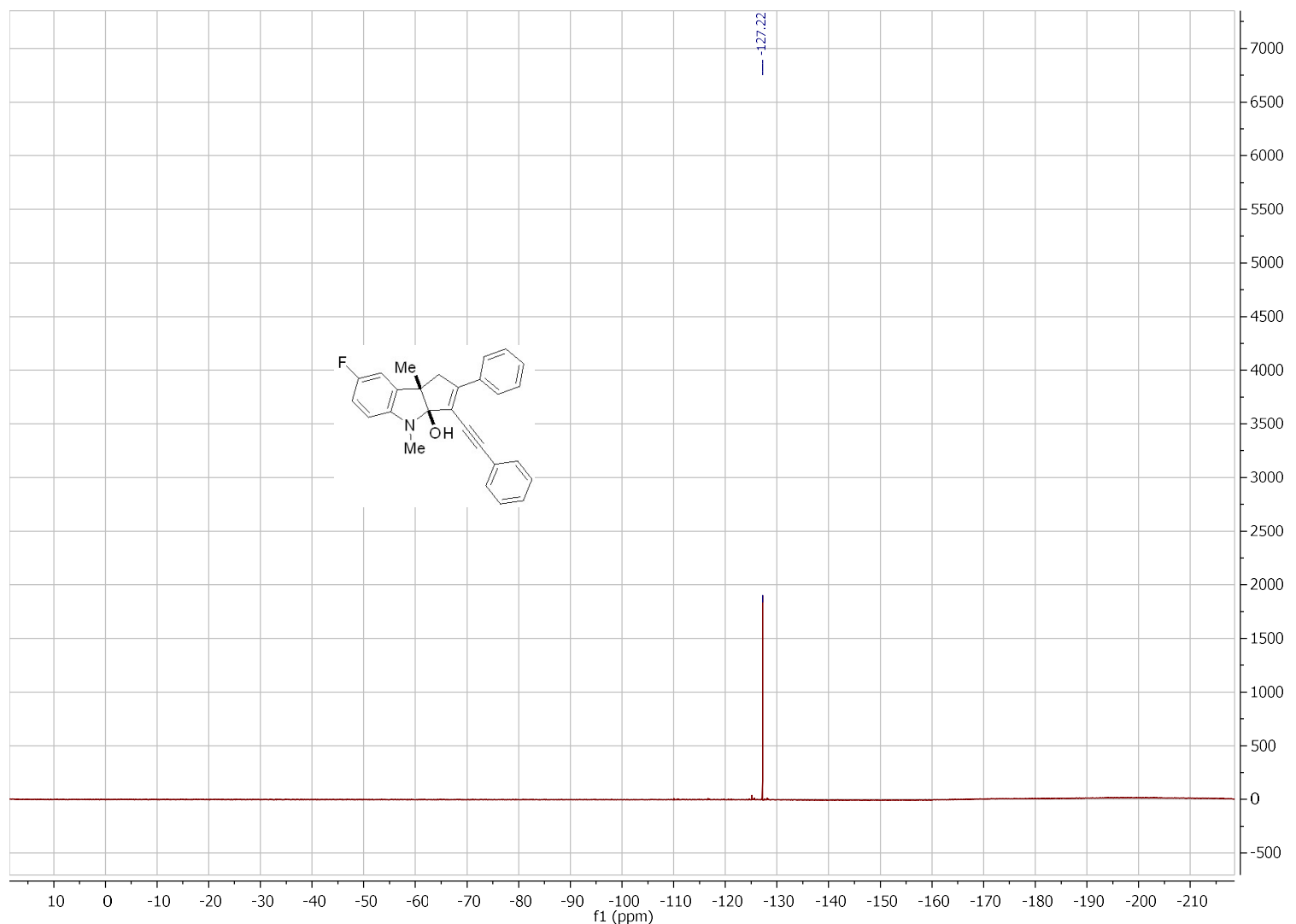




\section{3ca}
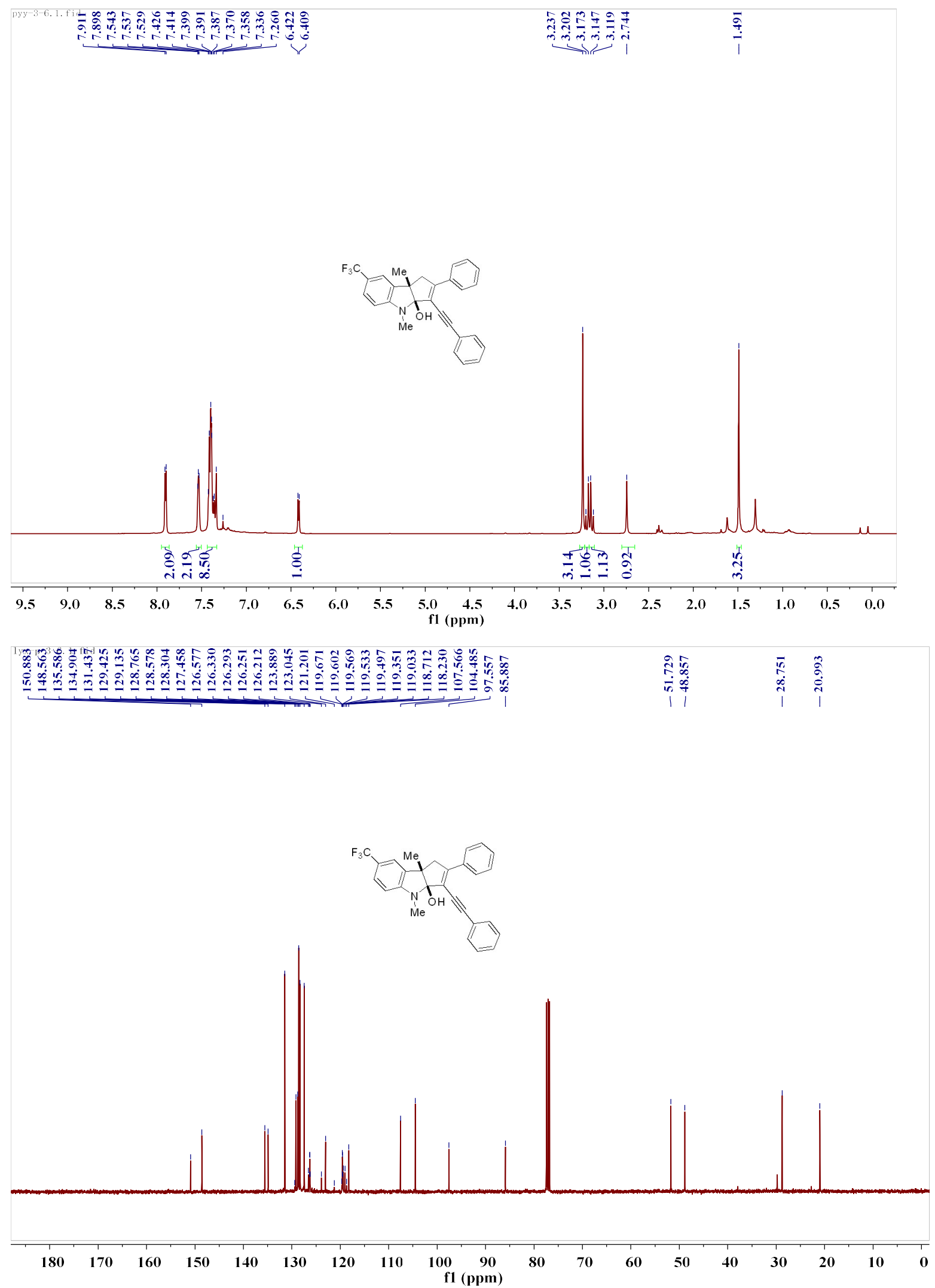


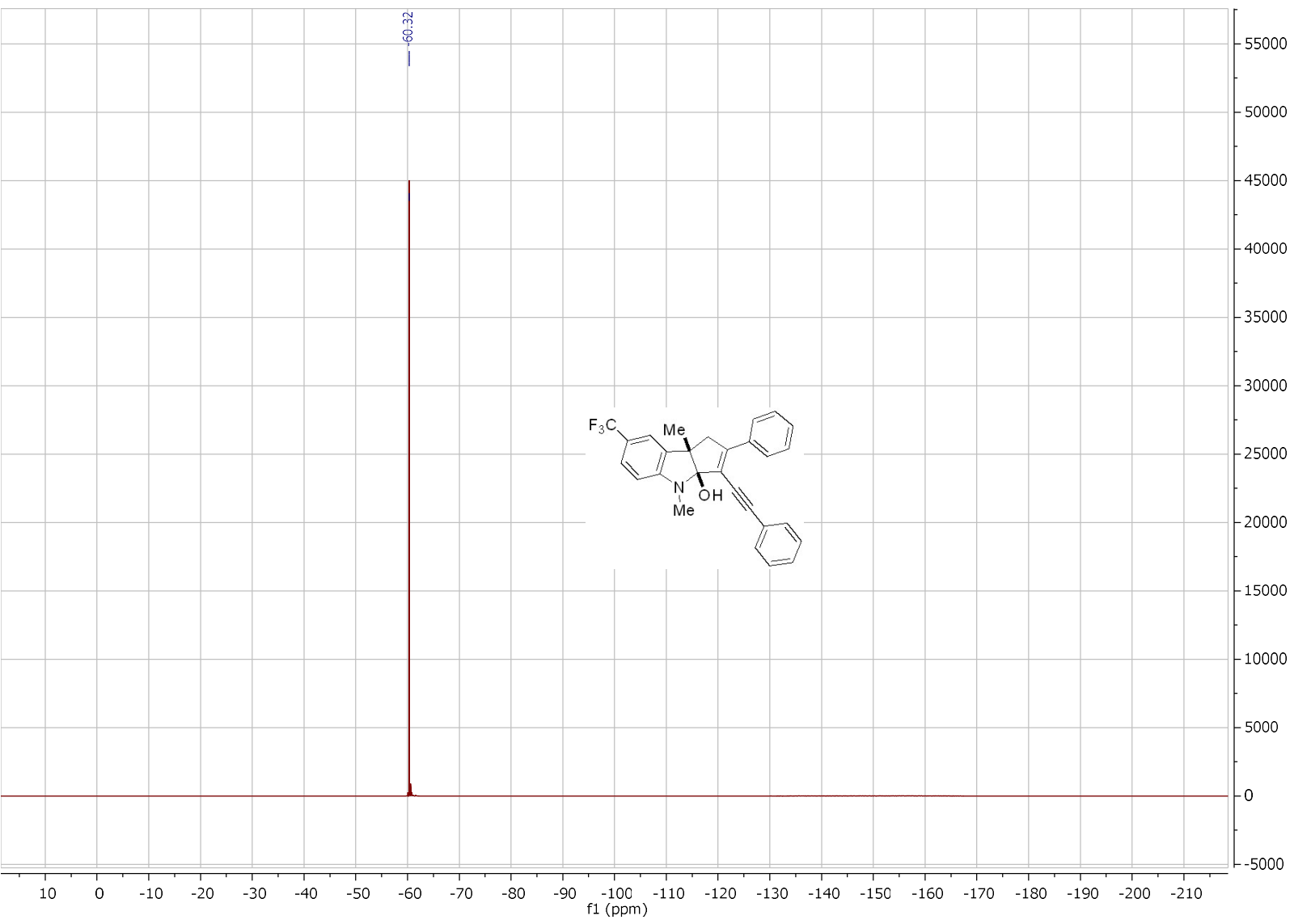


3da

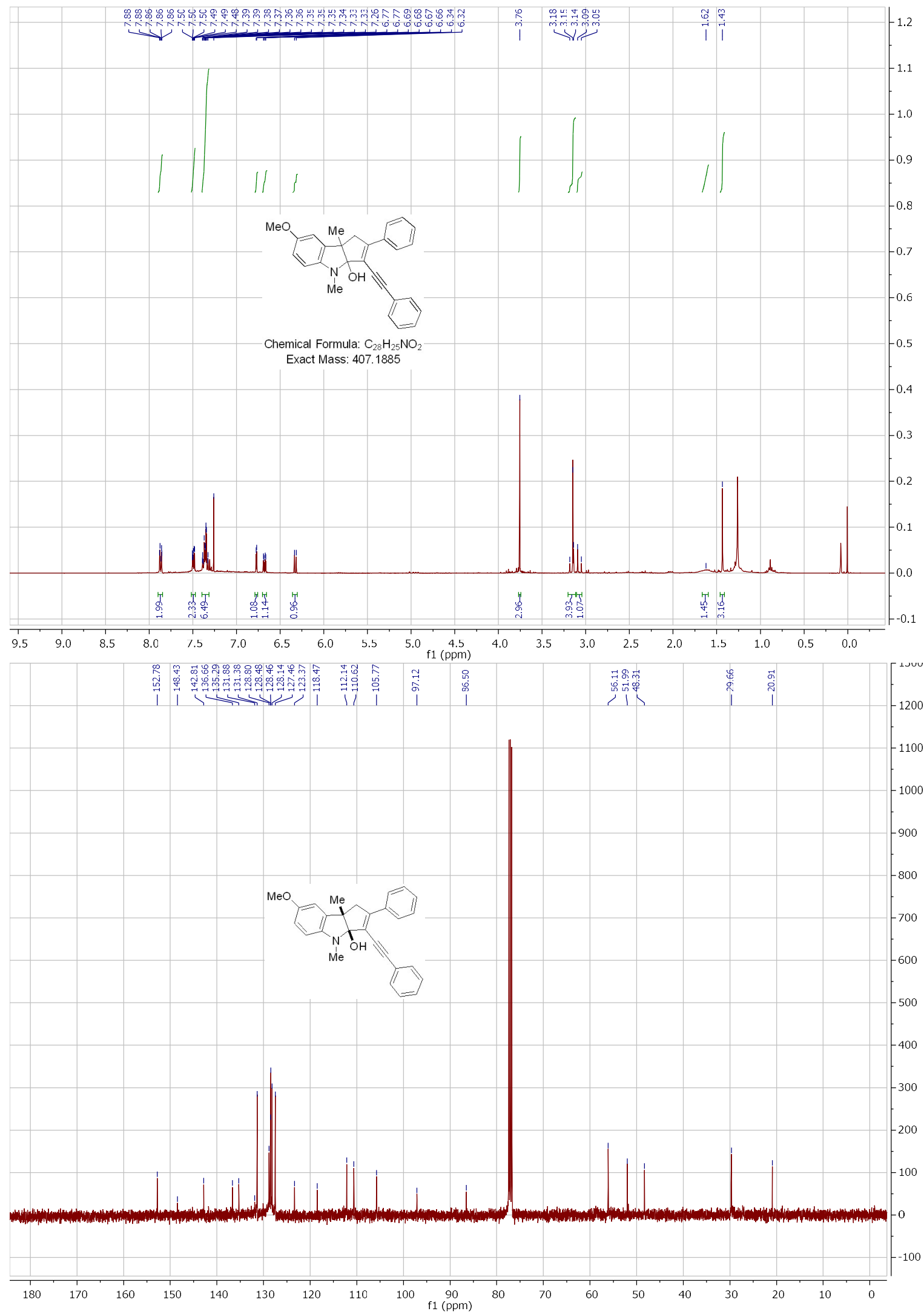


$3 f a$

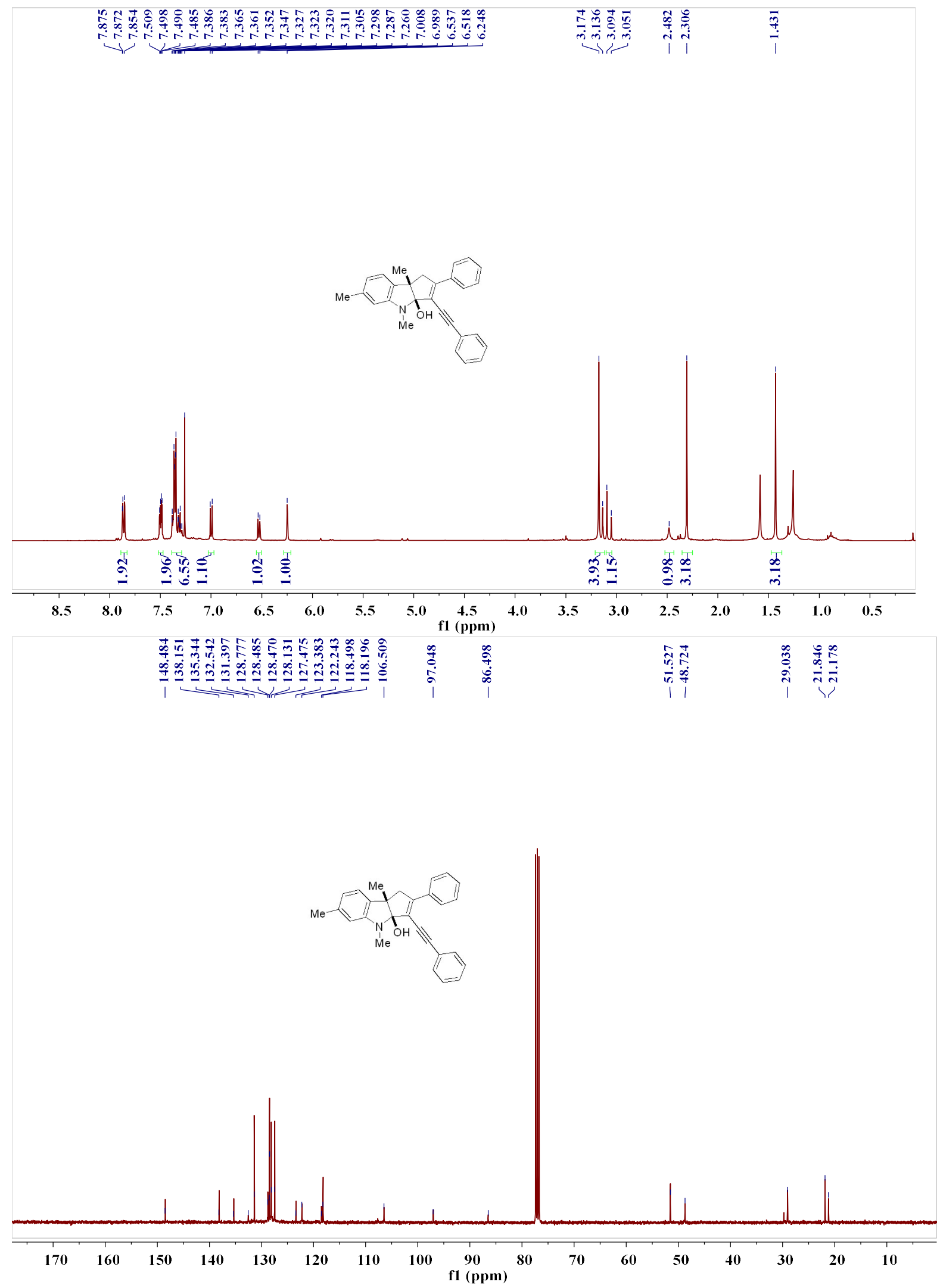




\section{$3 g a$}
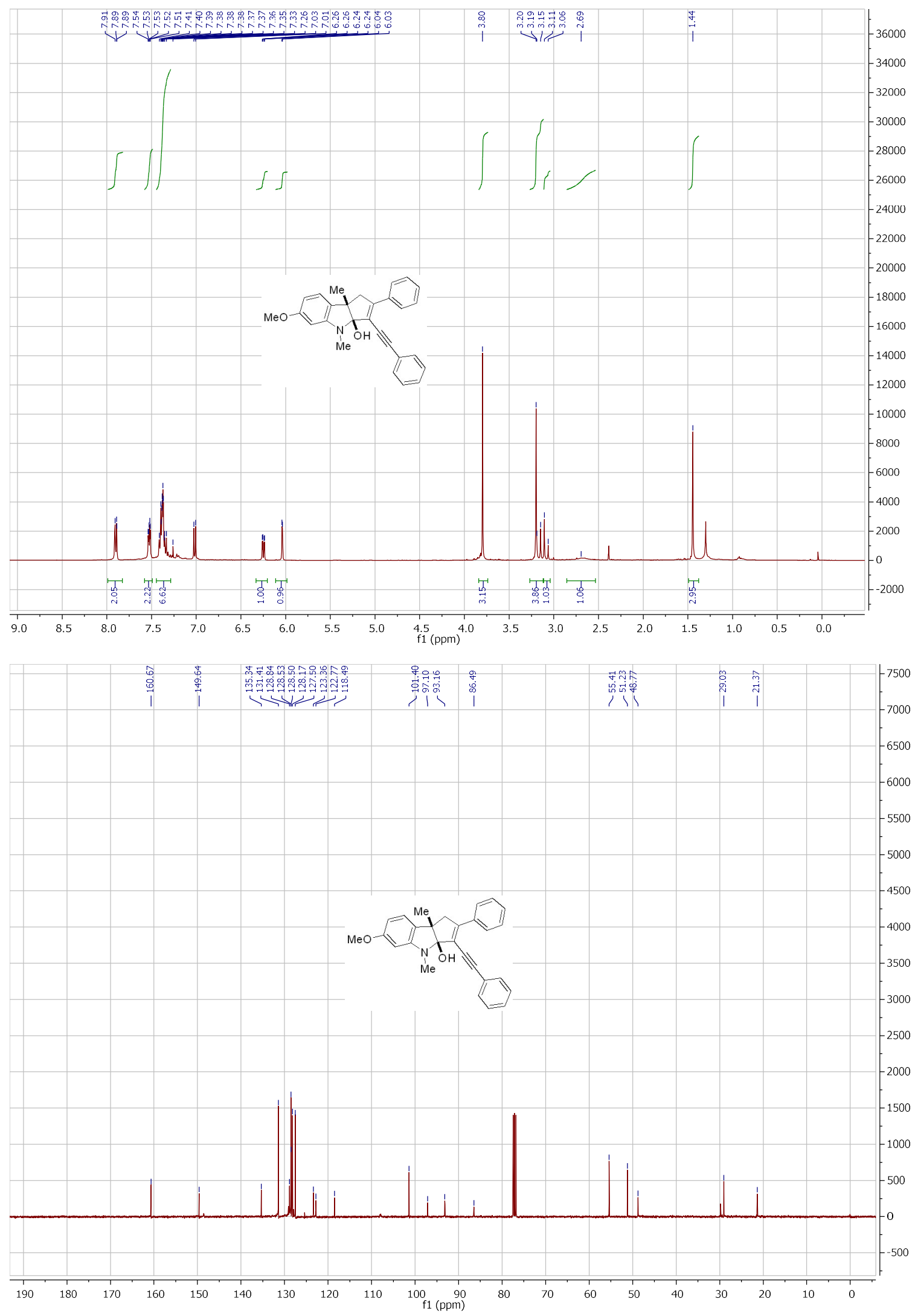
3ha

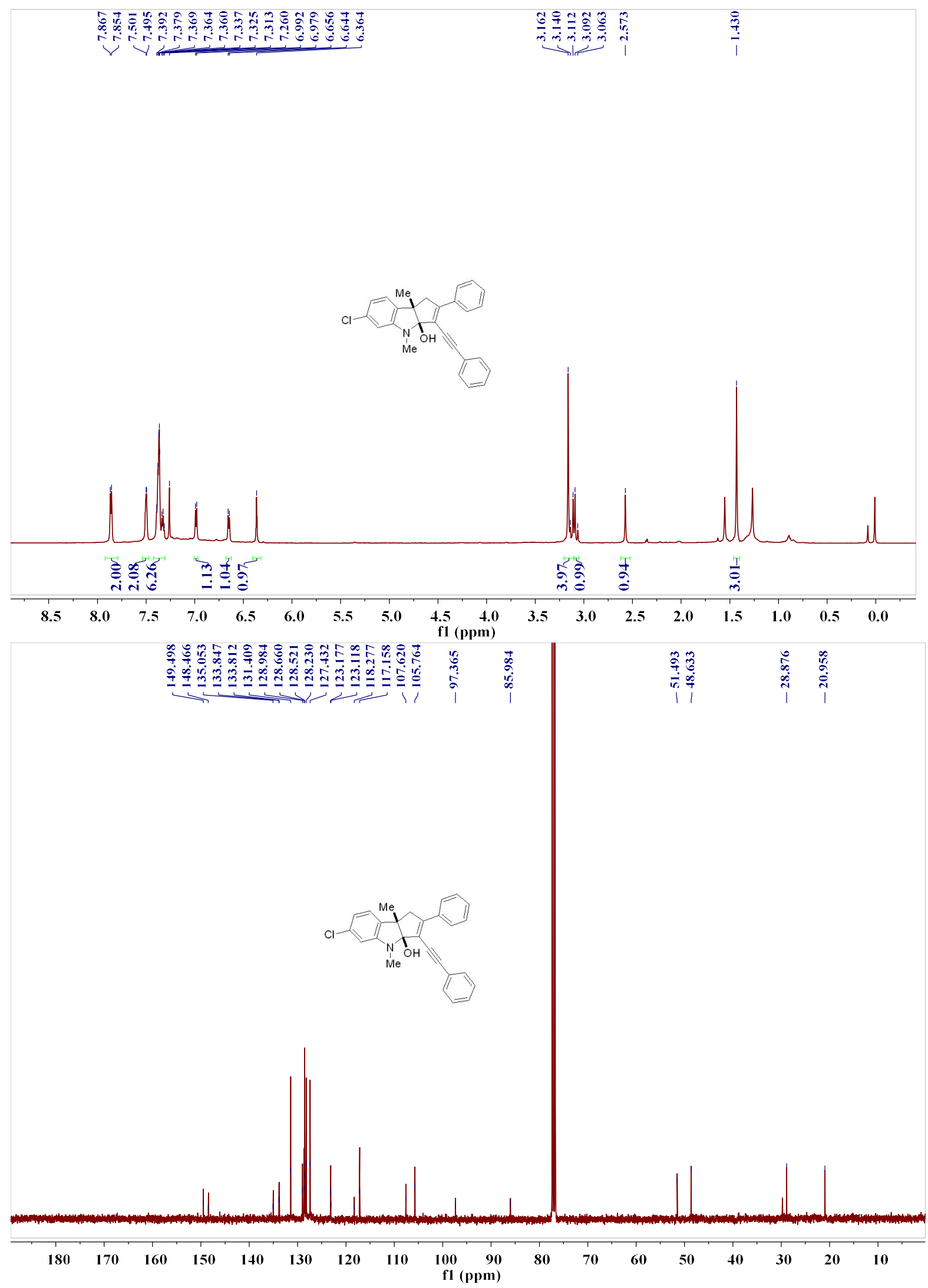




\section{3 ia}

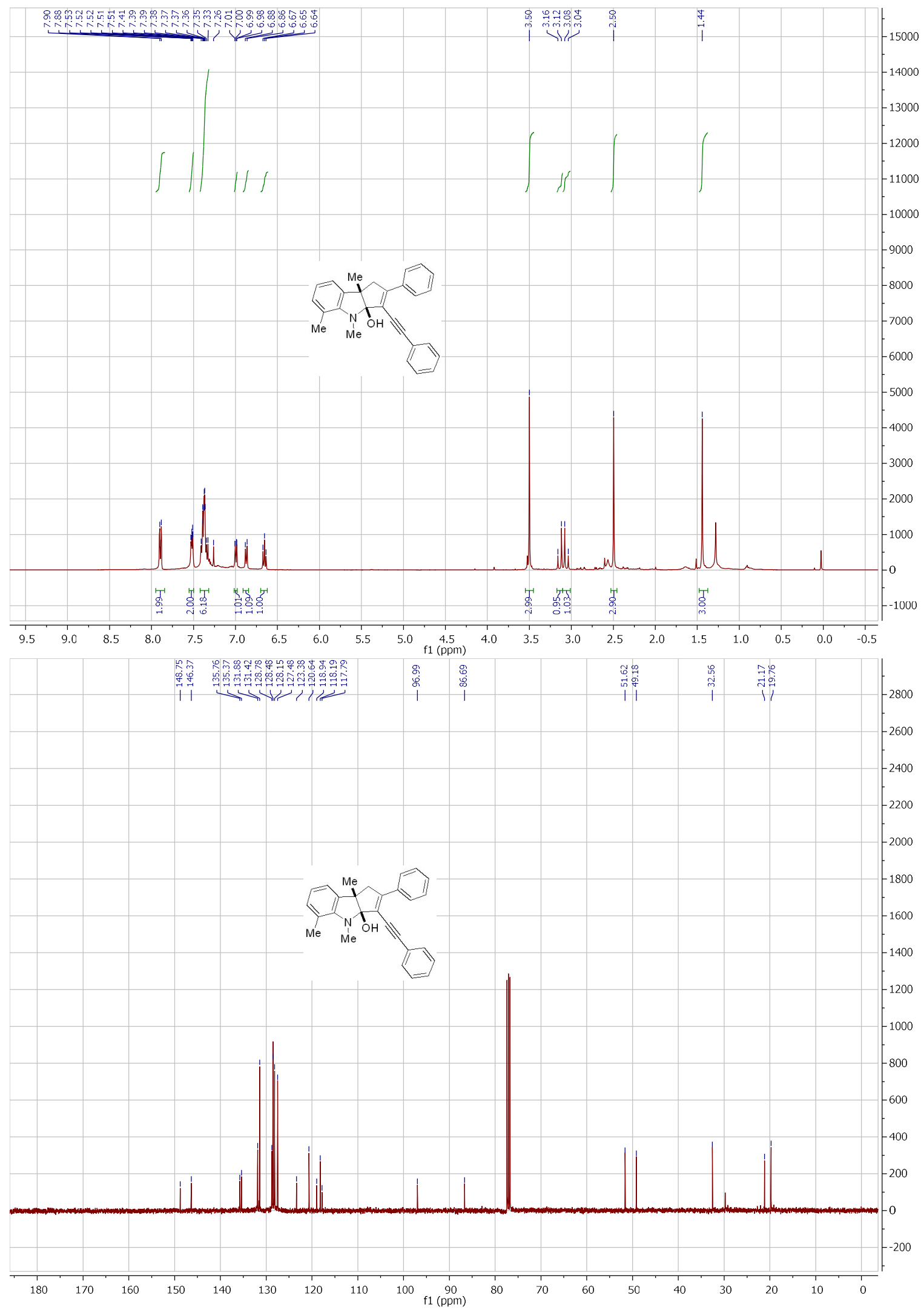


3ja
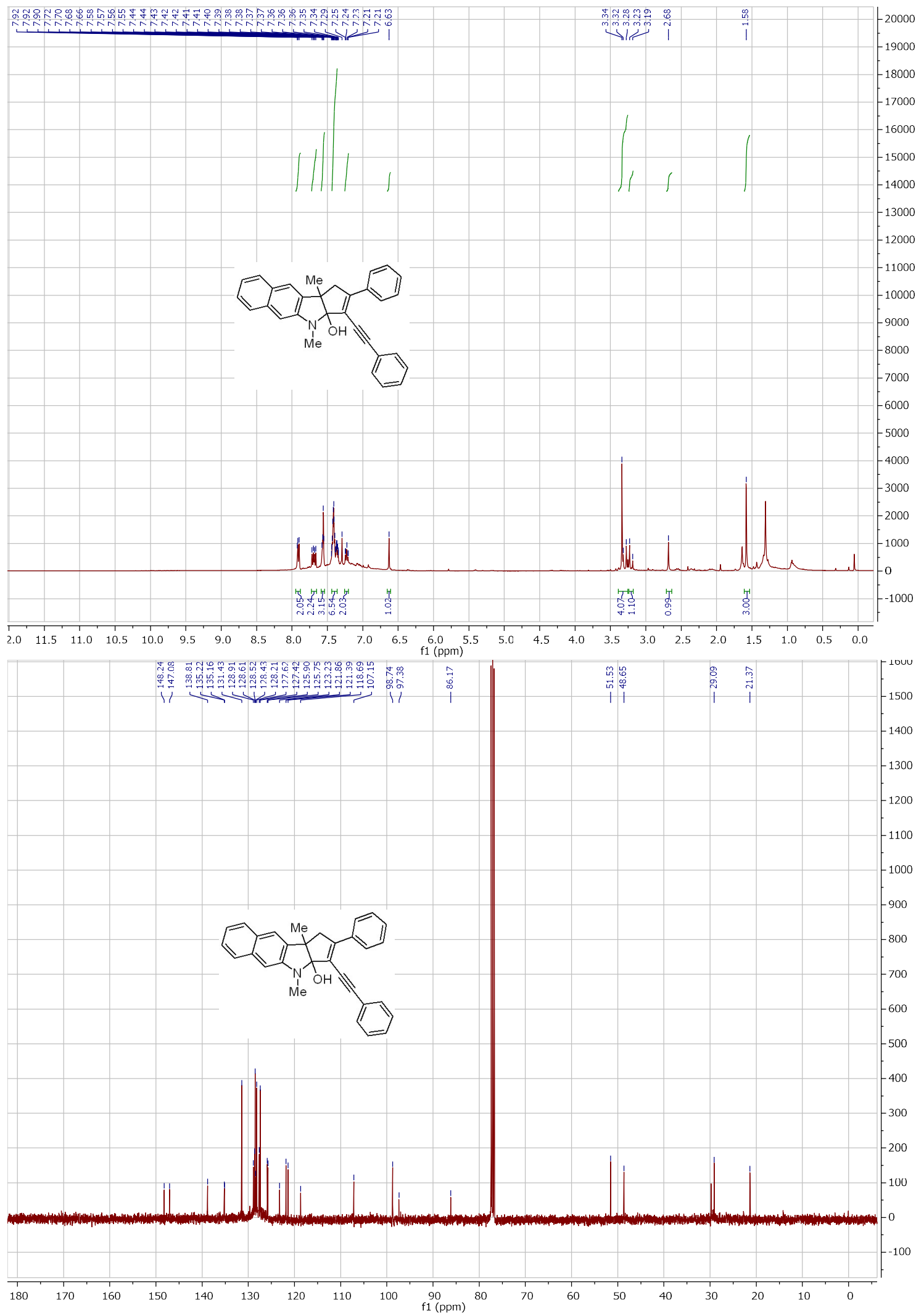
3ka

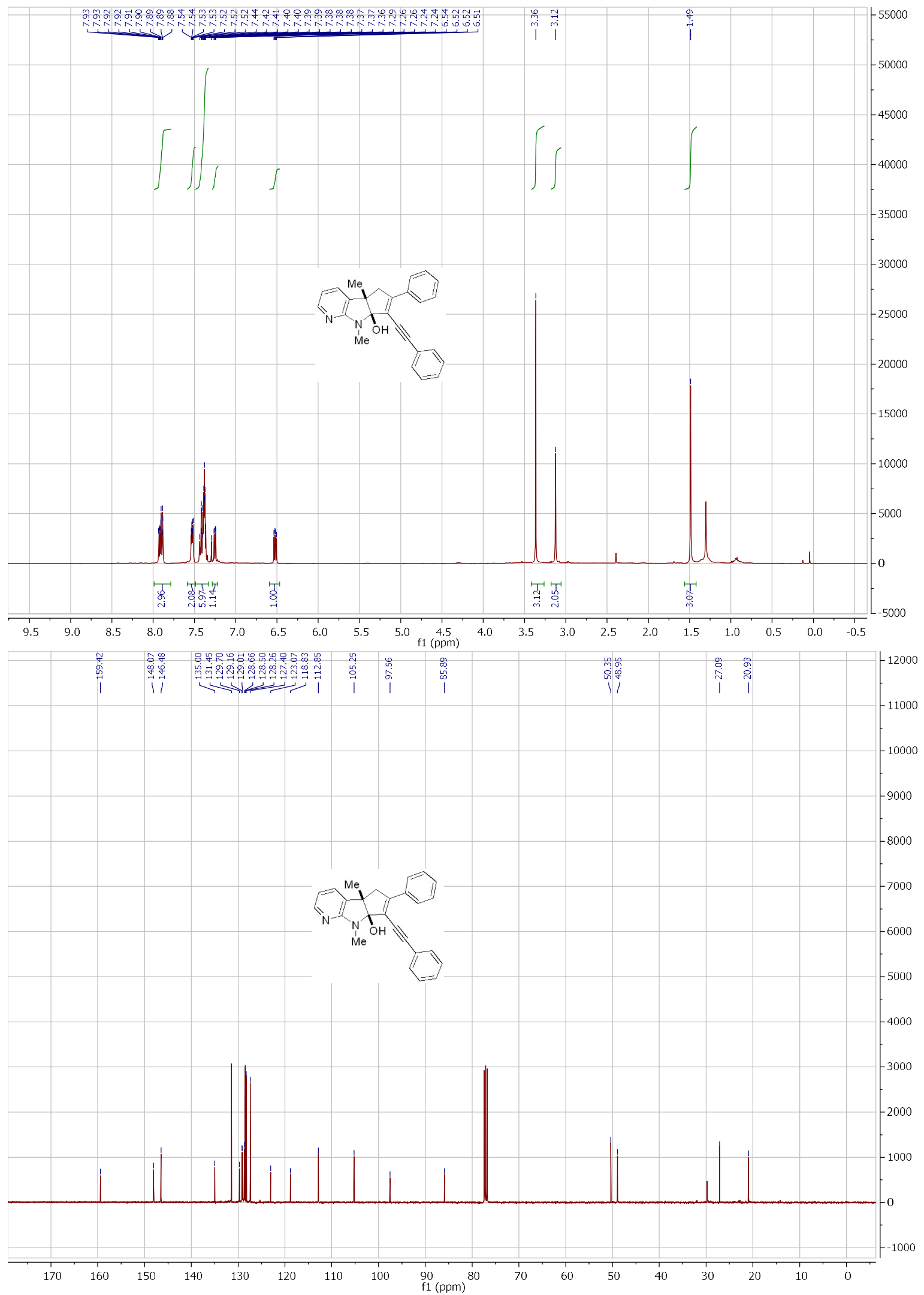




\section{3ma}

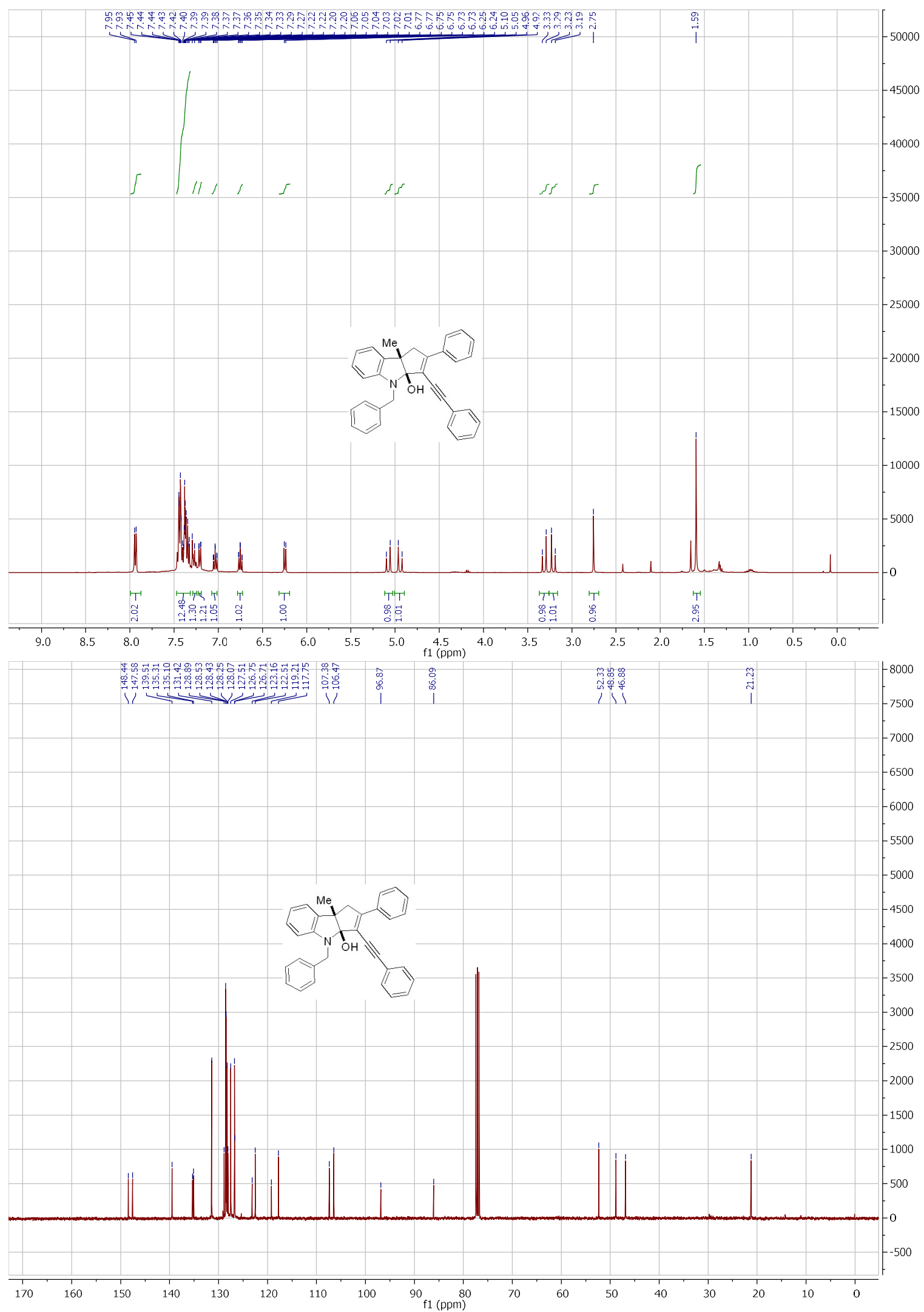




\section{3na}

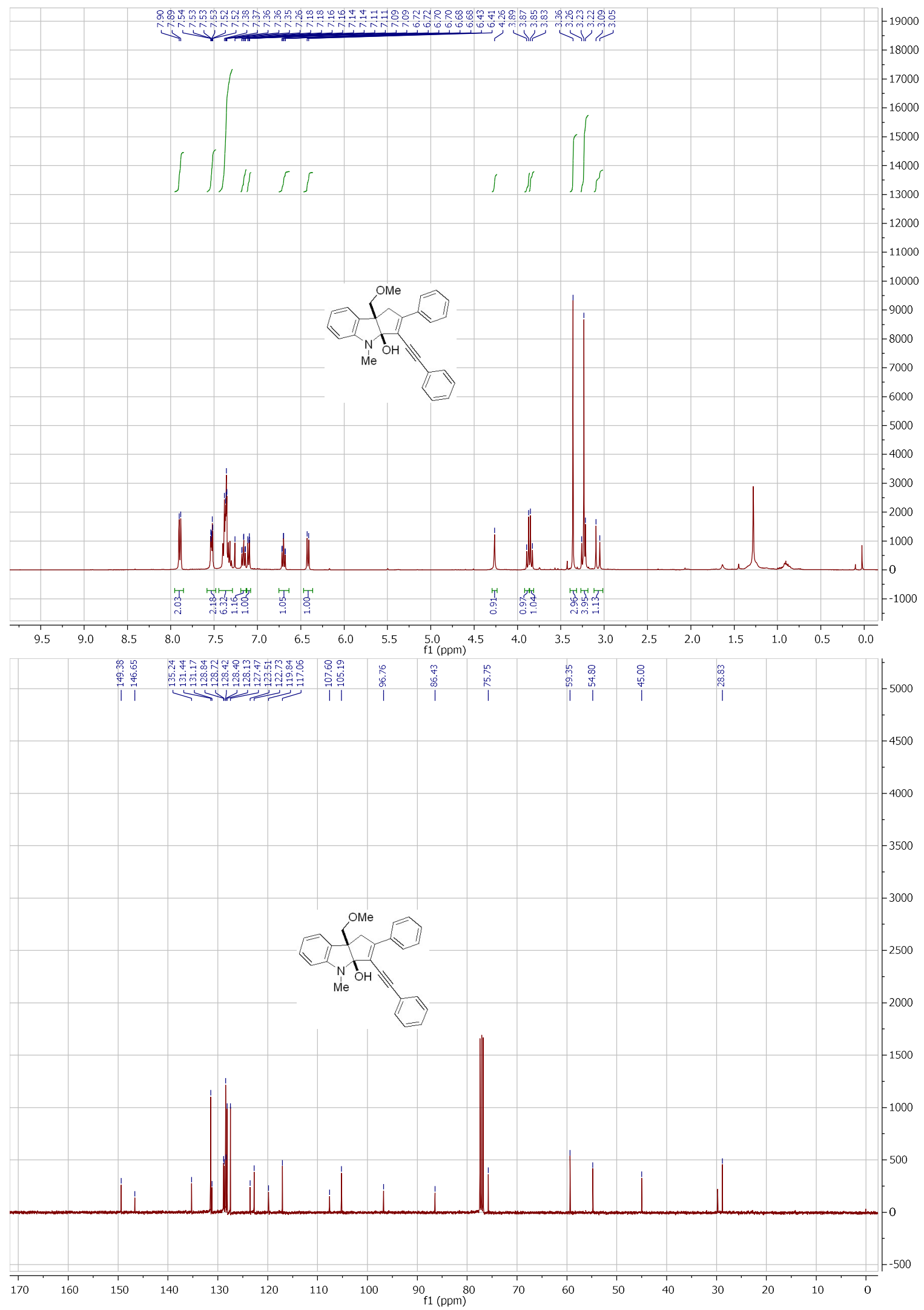


$30 a$

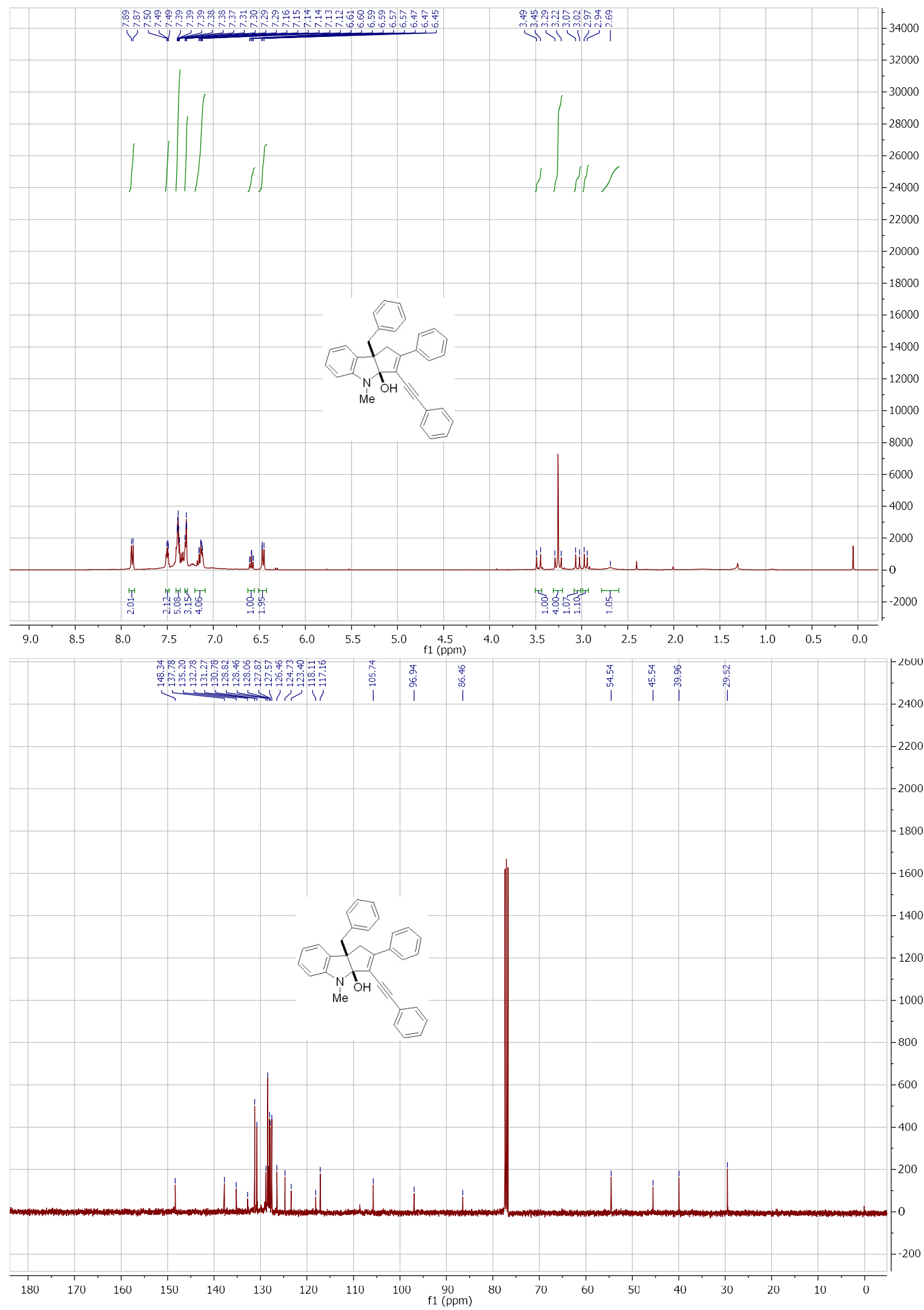




\section{$3 p a$}
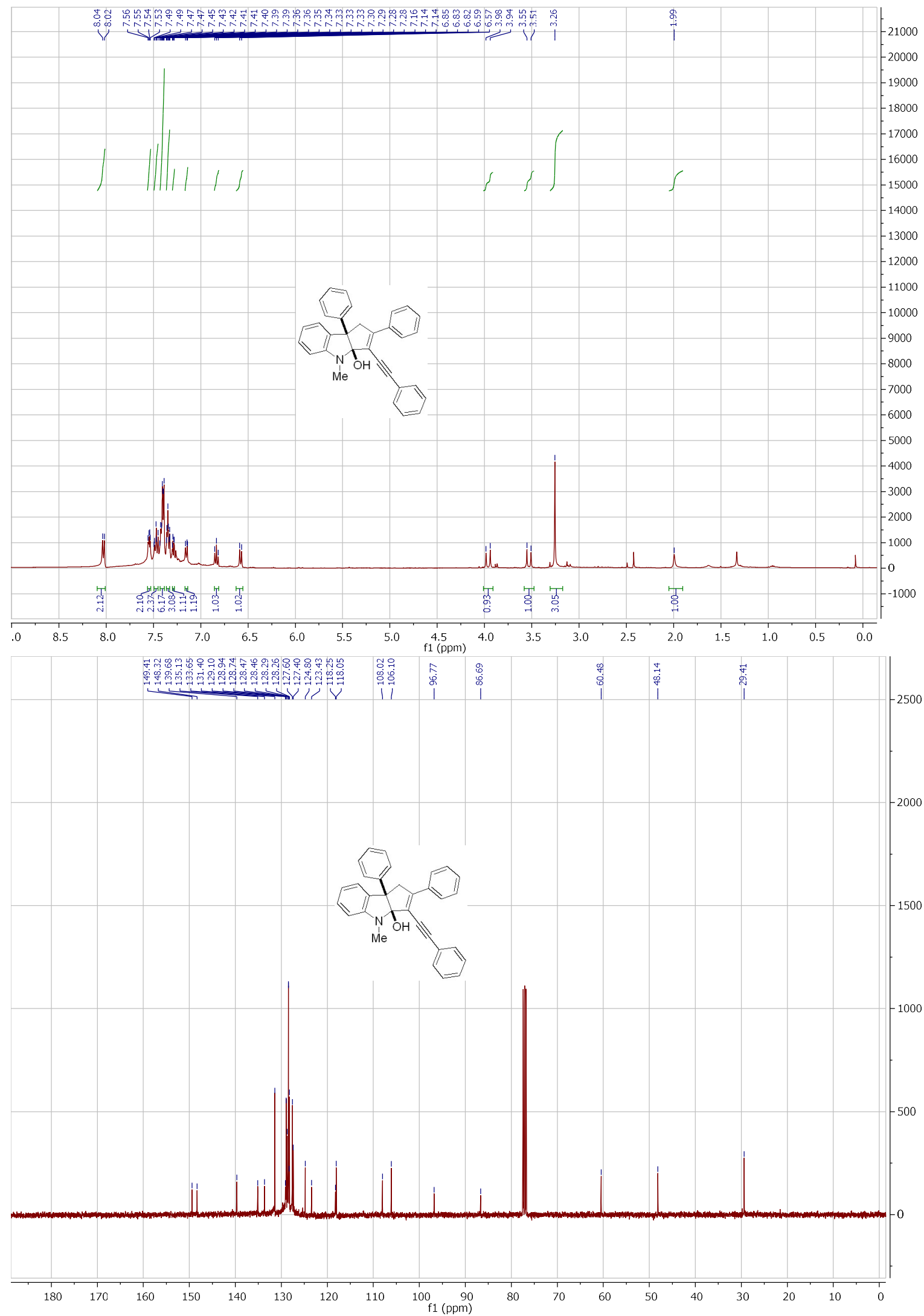
$3 a b$

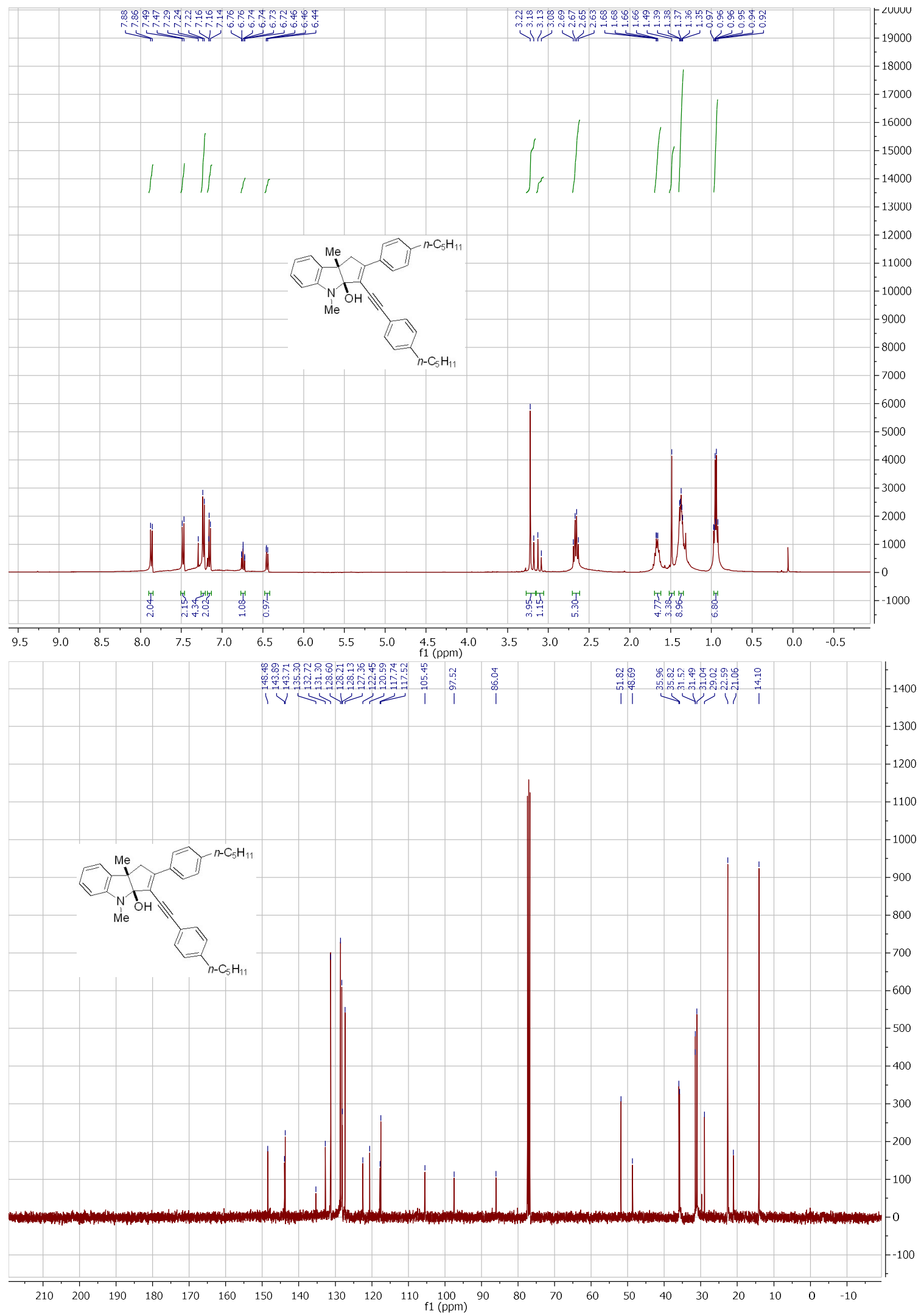




\section{$3 a c$}
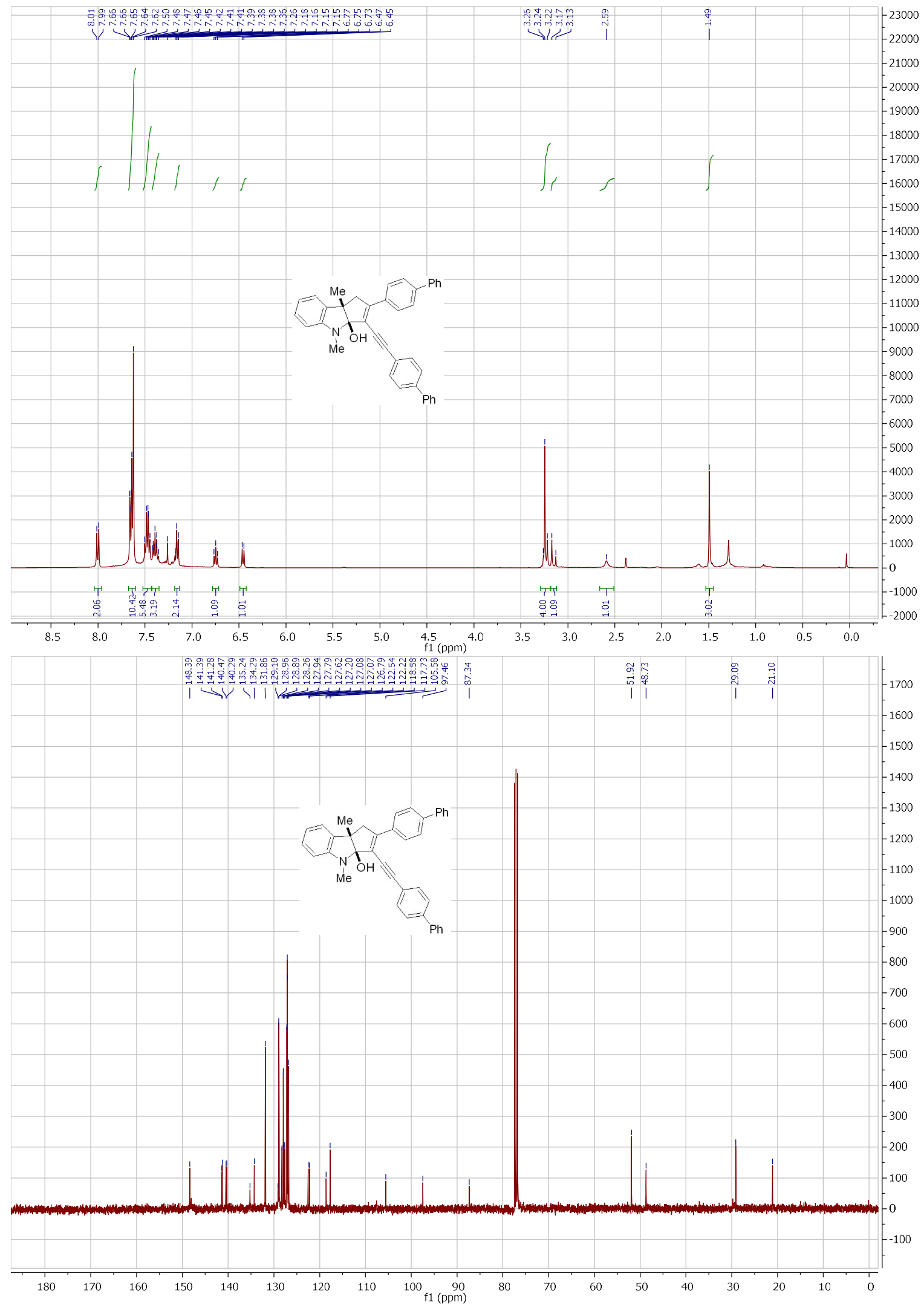


\section{3ad}

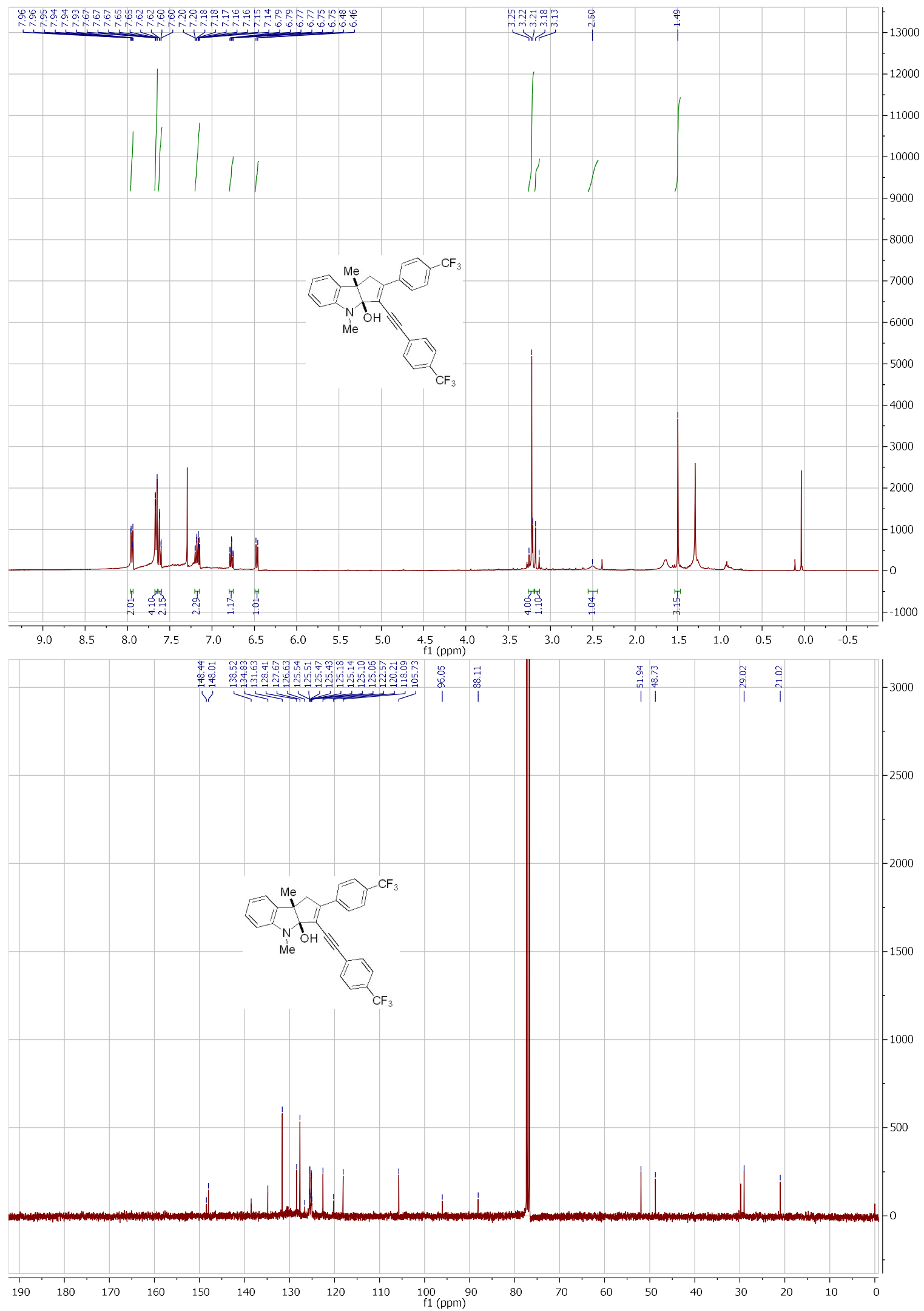




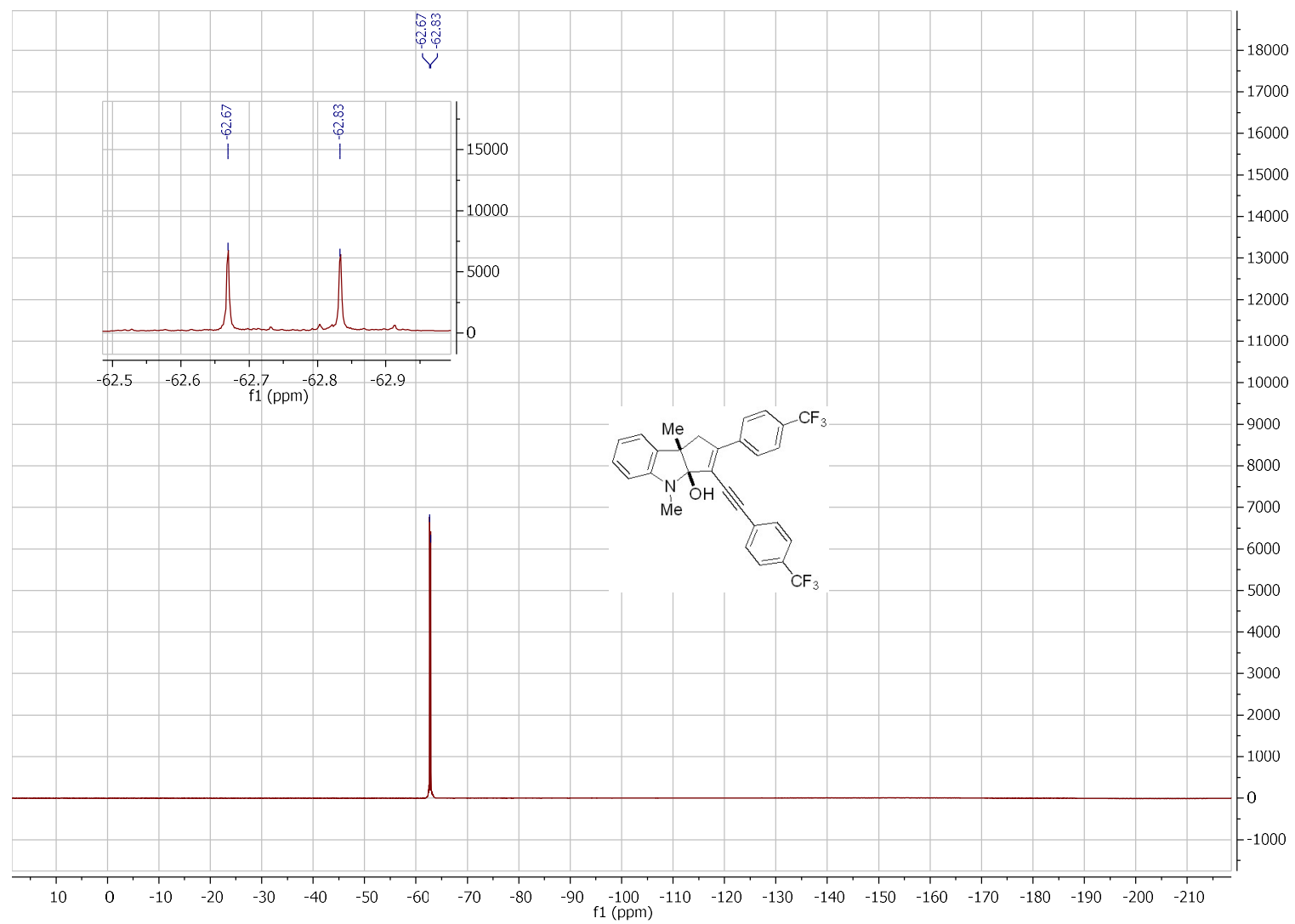




\section{$3 a e$}
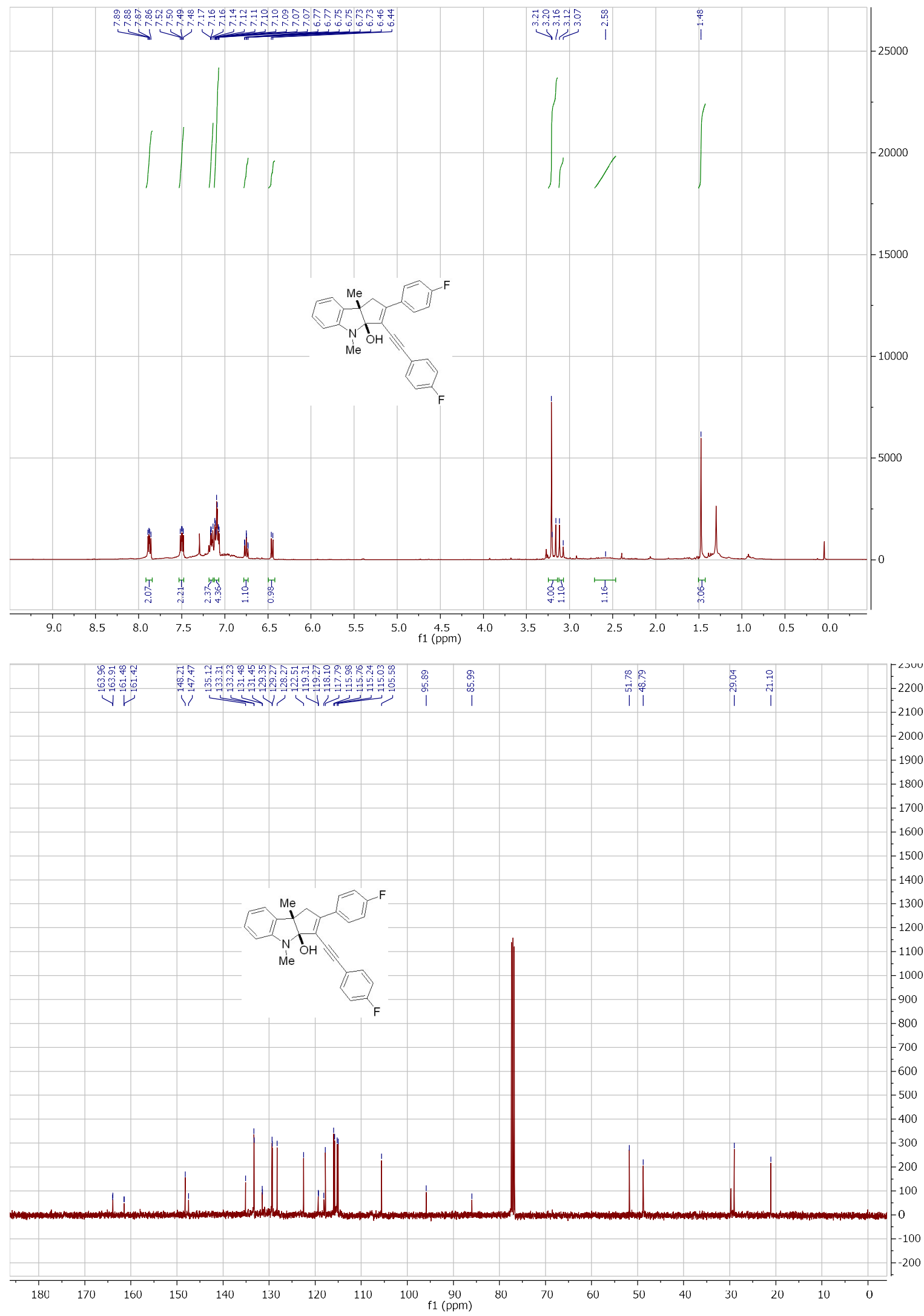


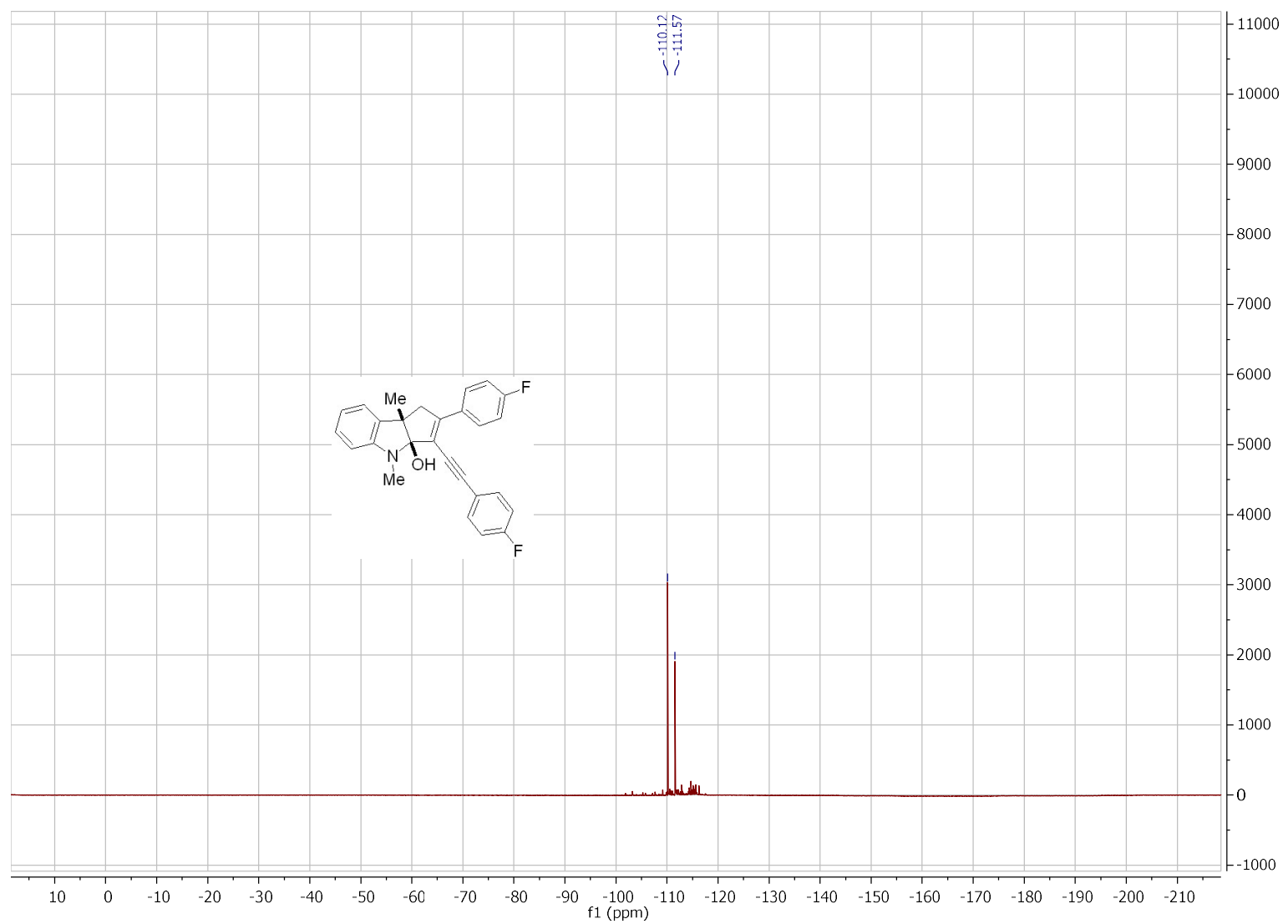


3af

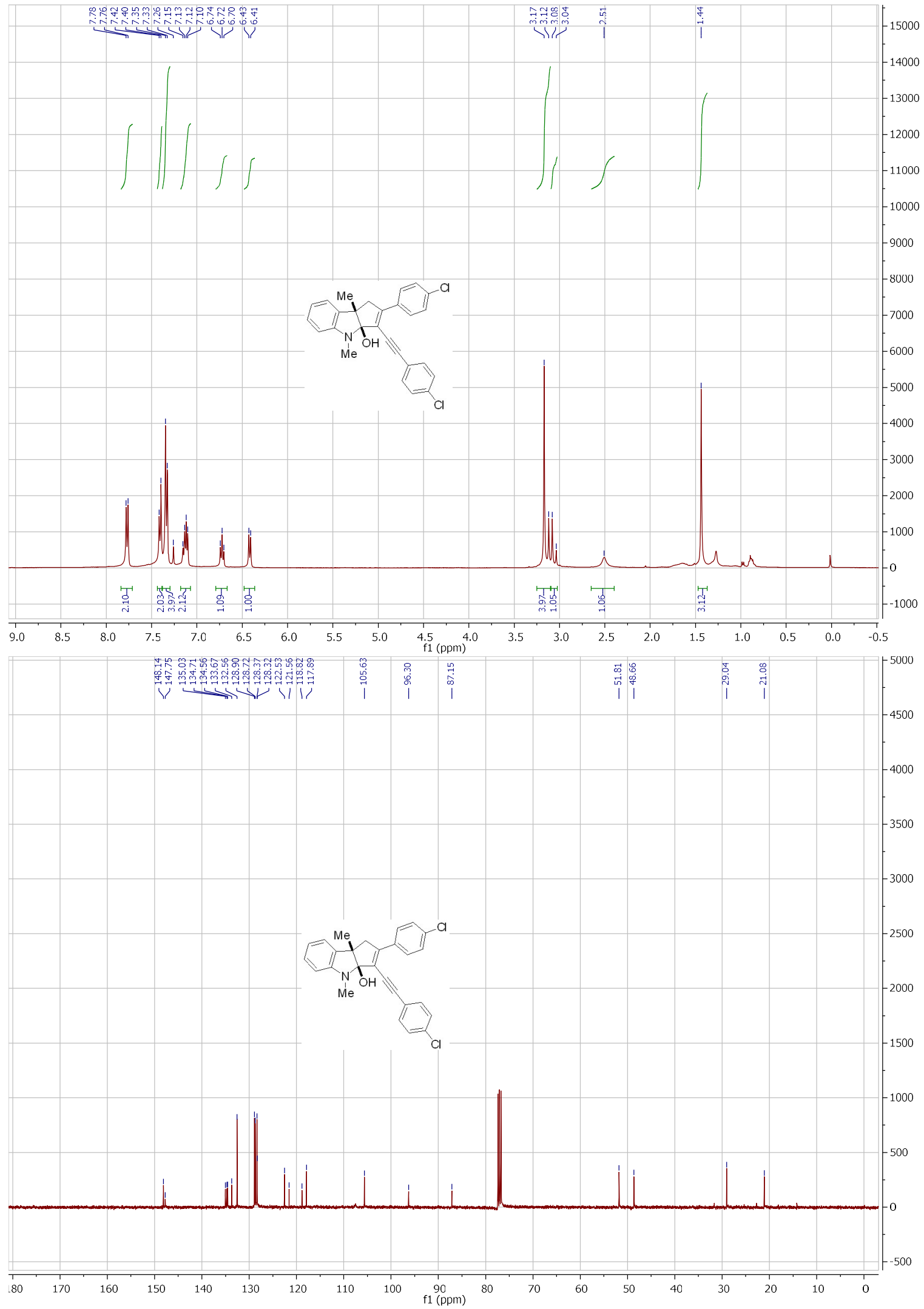


3ag

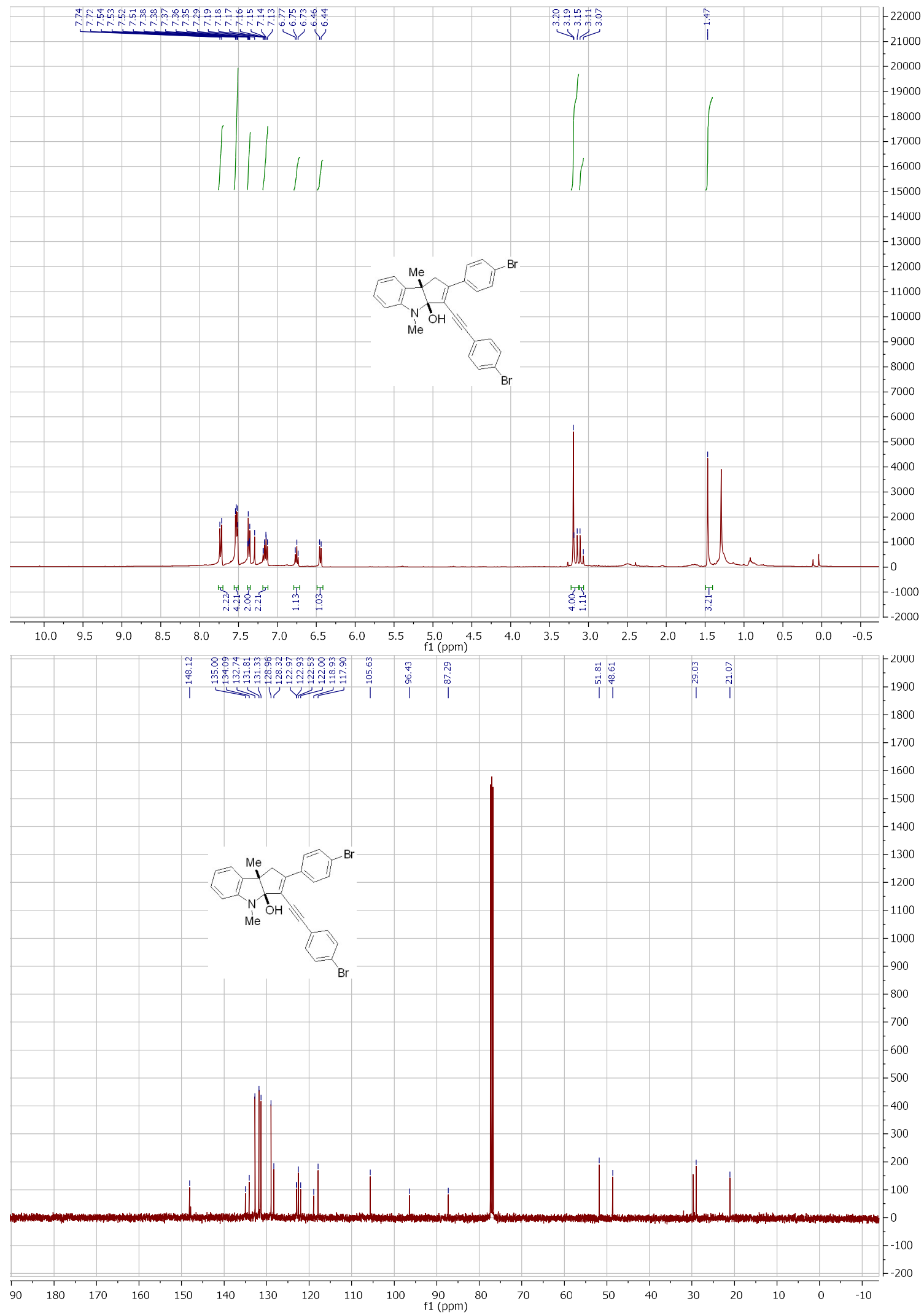


3ah

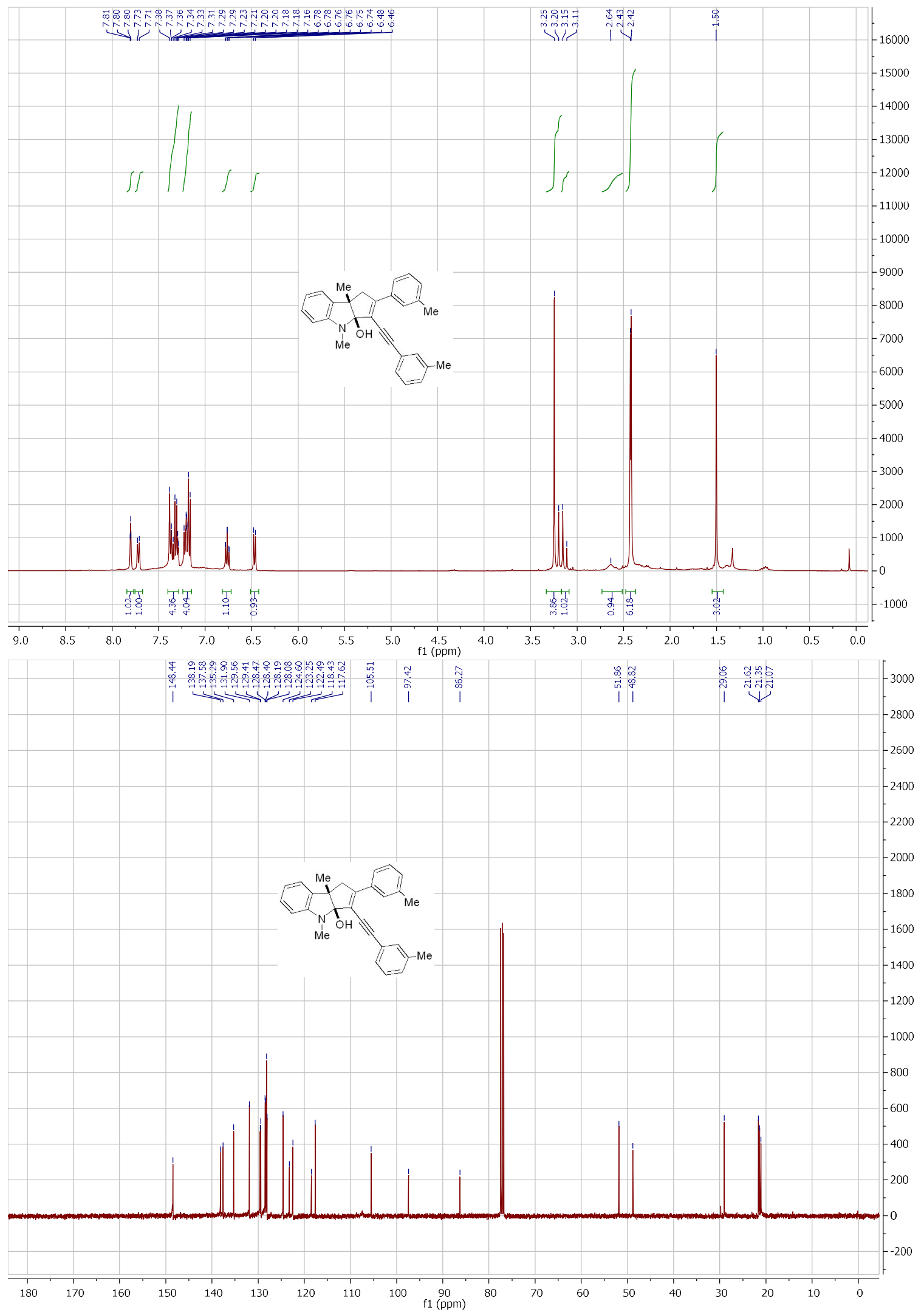


3ai
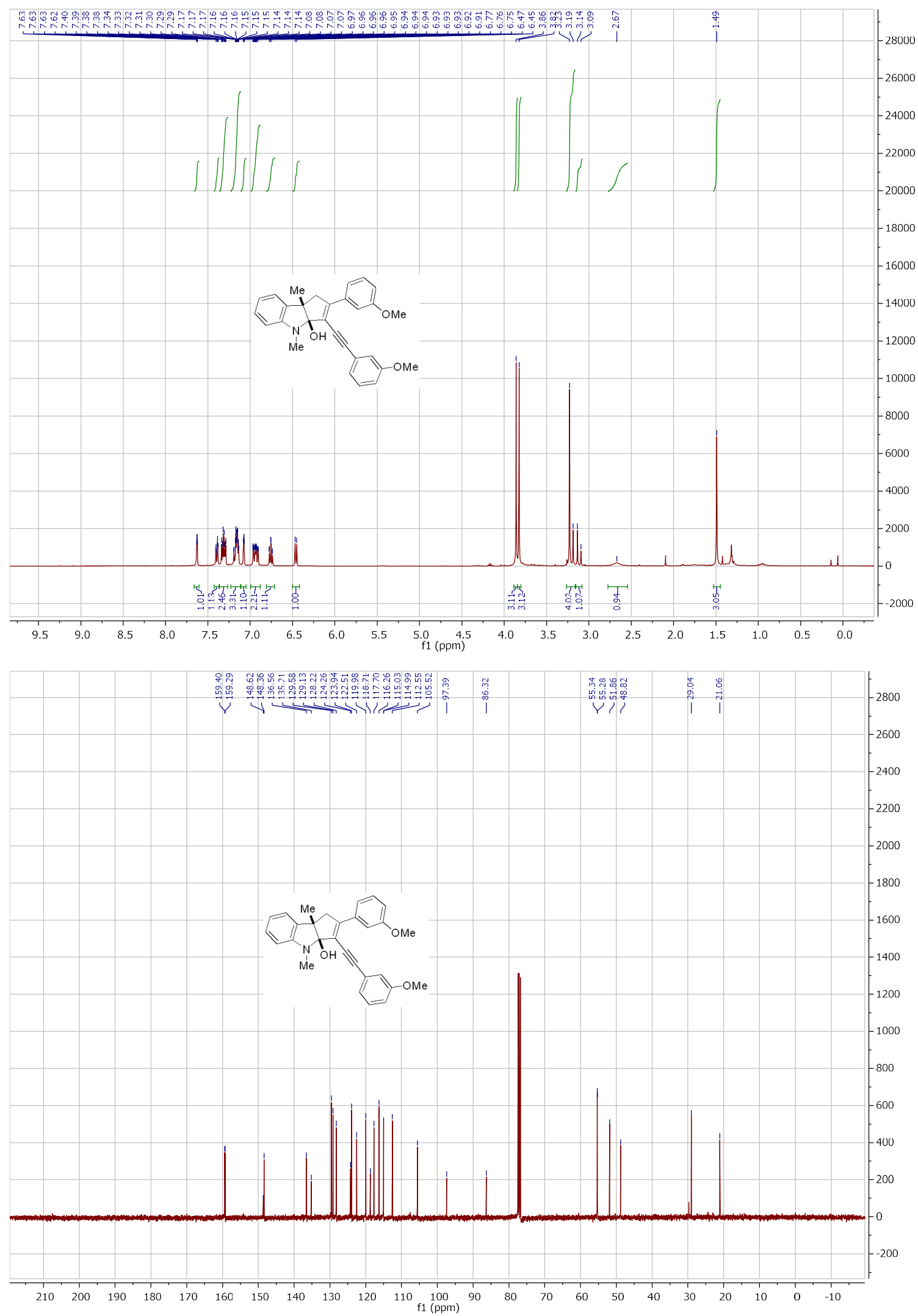
3aj
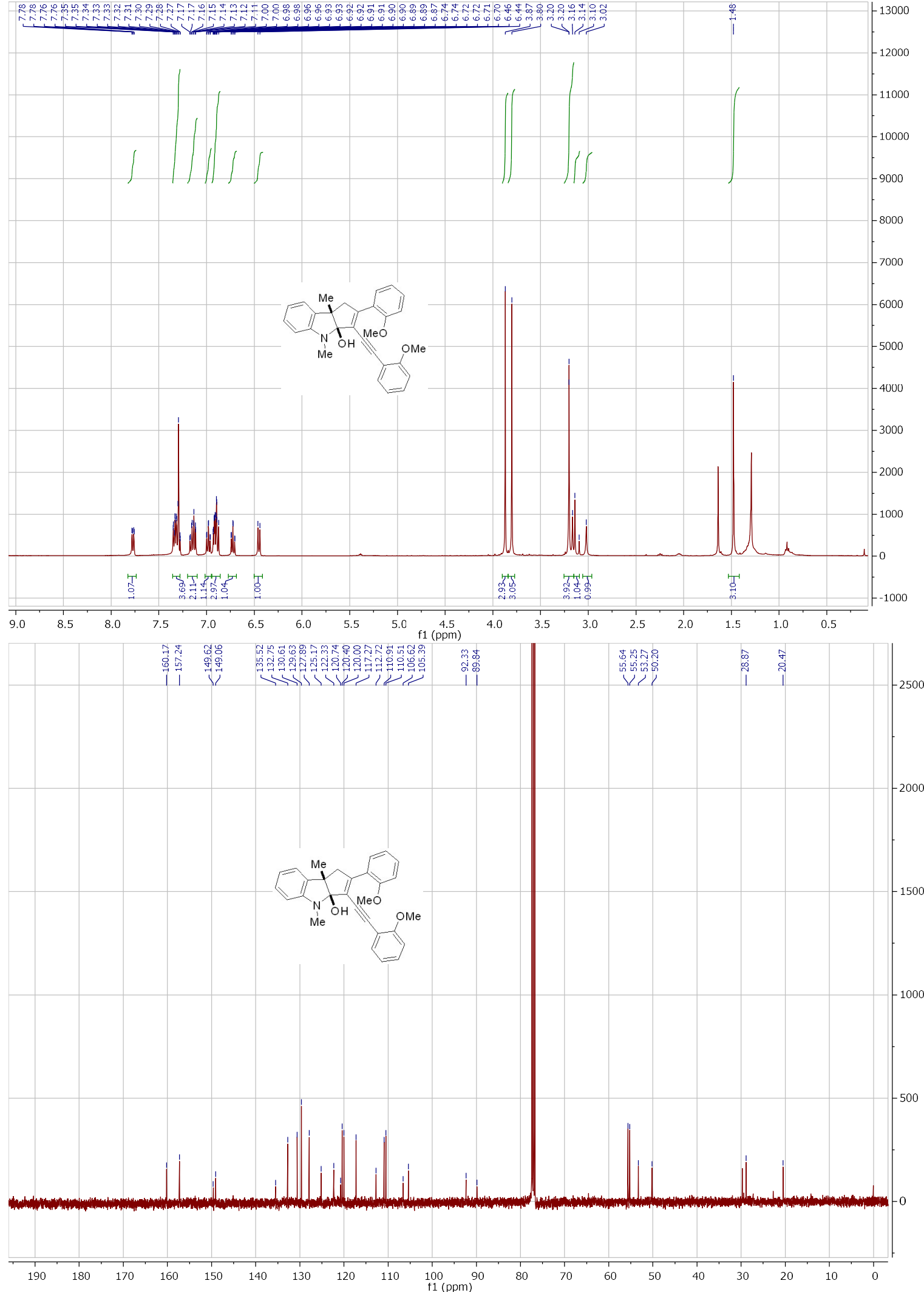


\section{3ak}

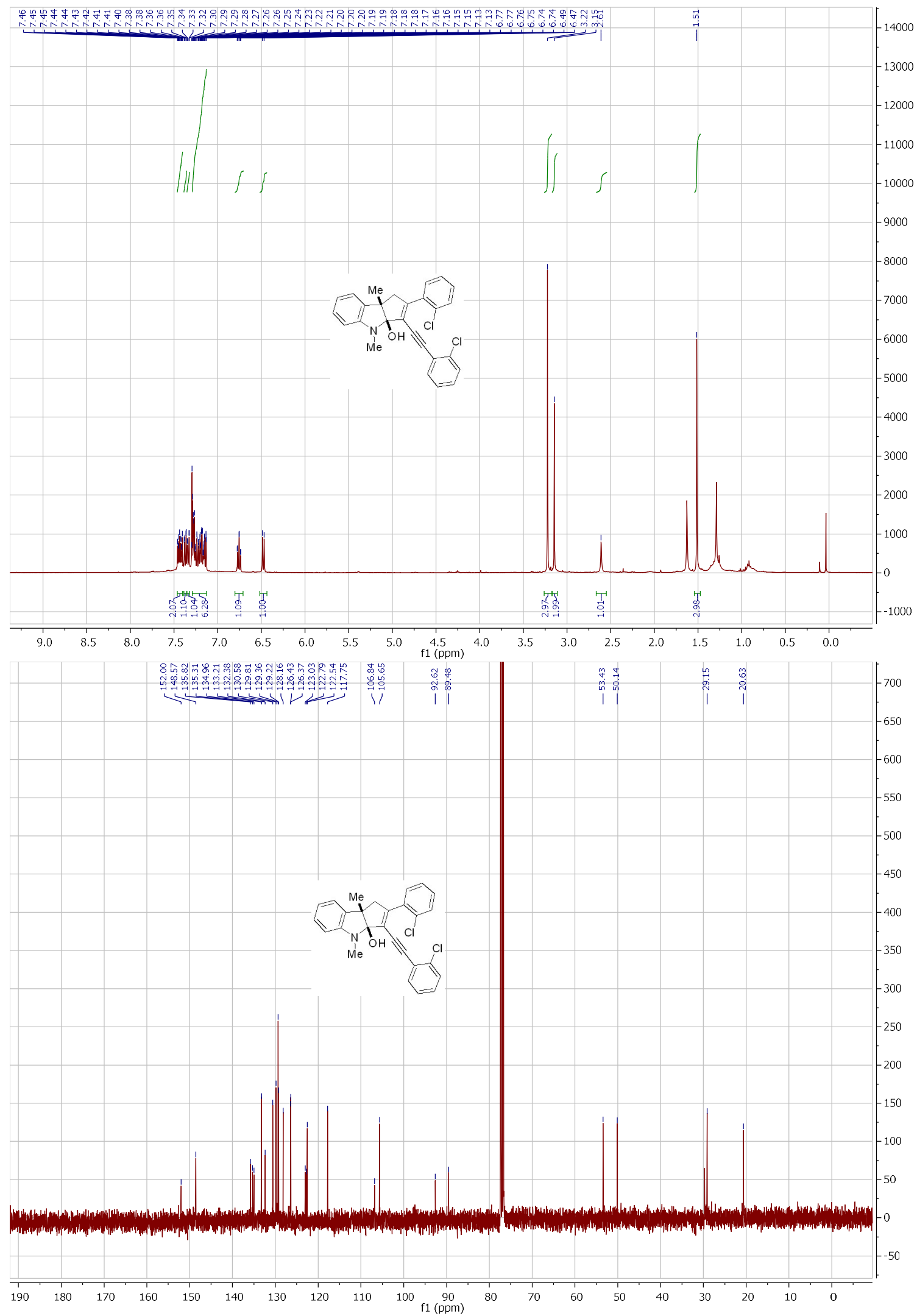


3al

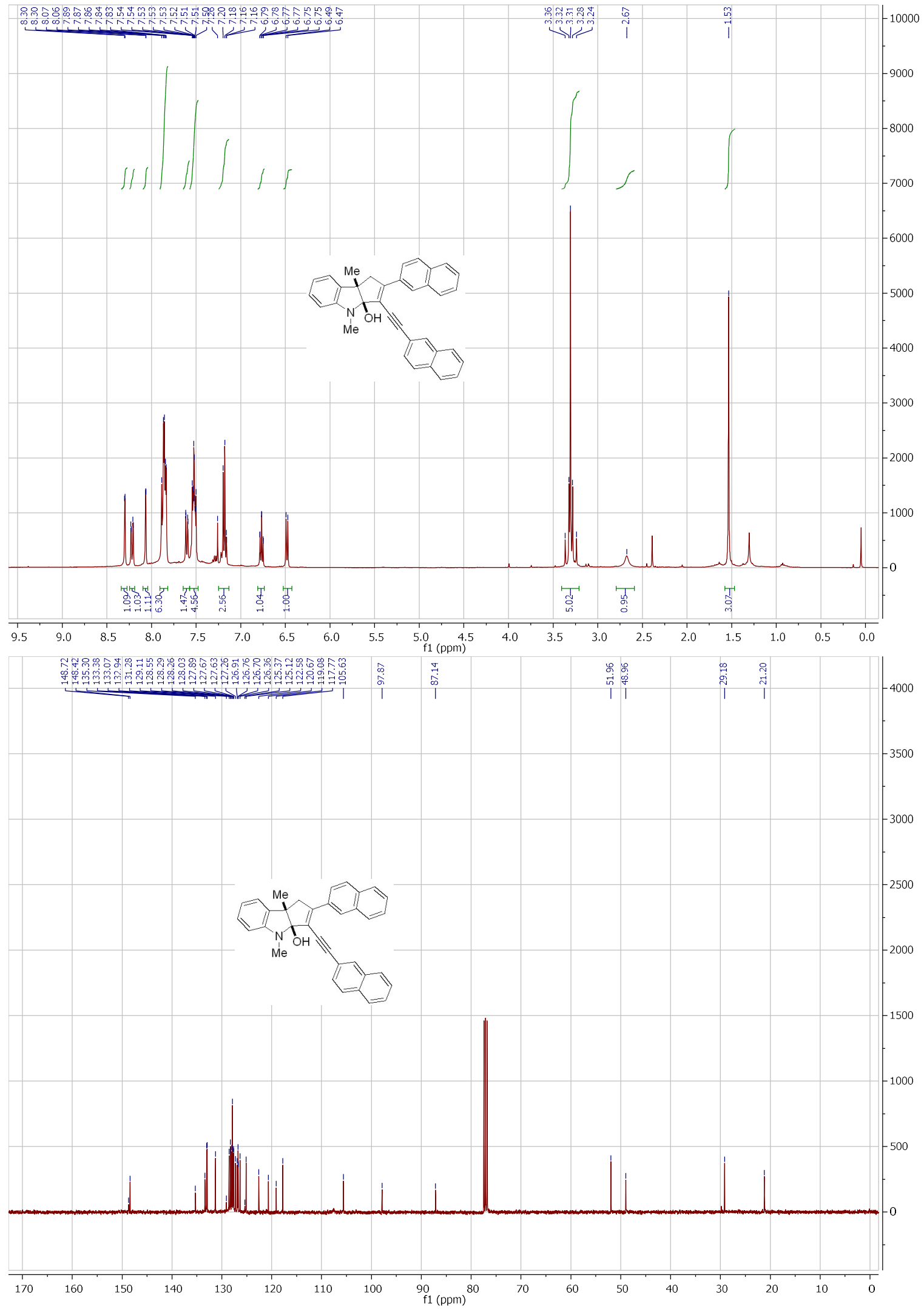




\section{3am}
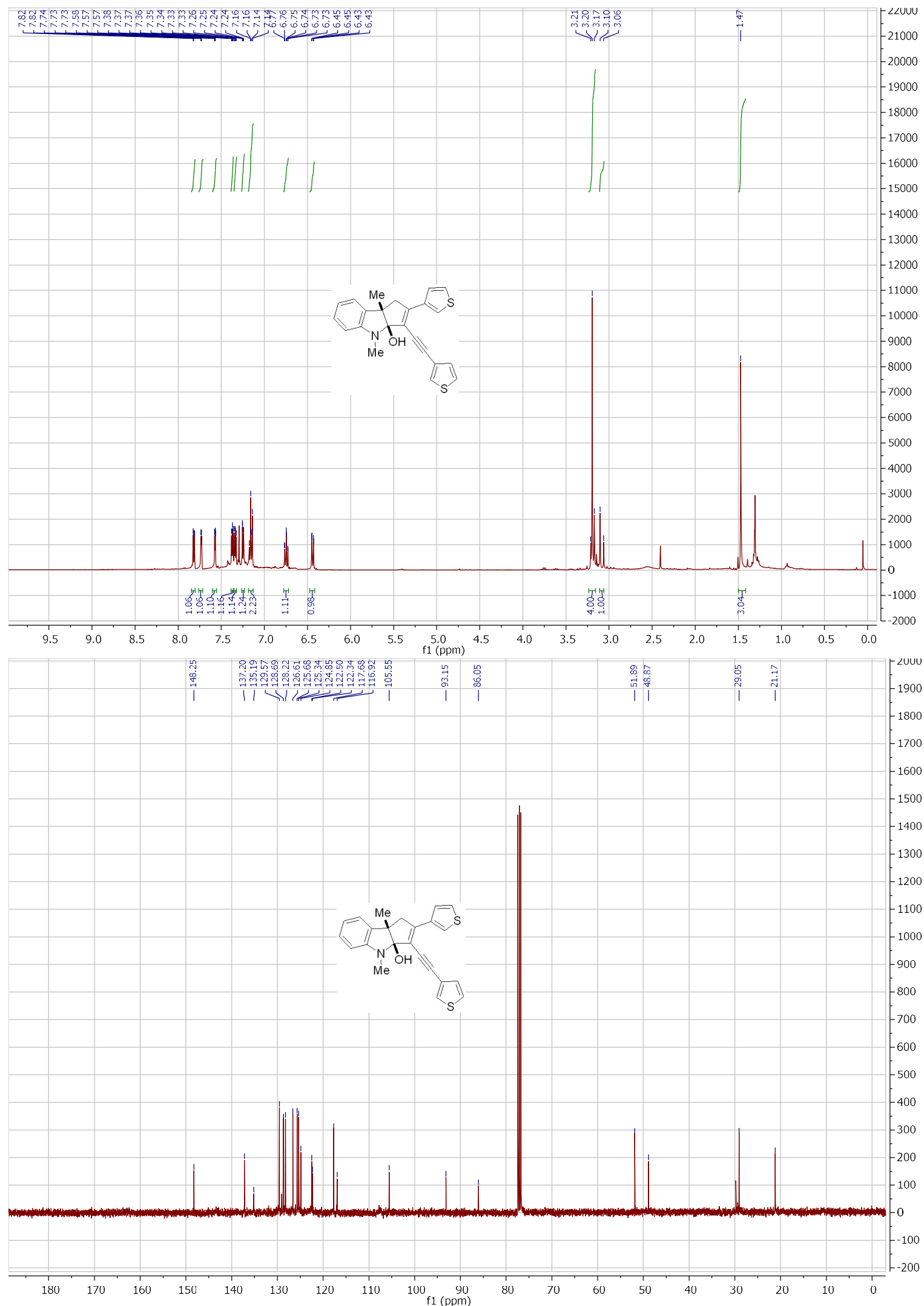


\section{3an}

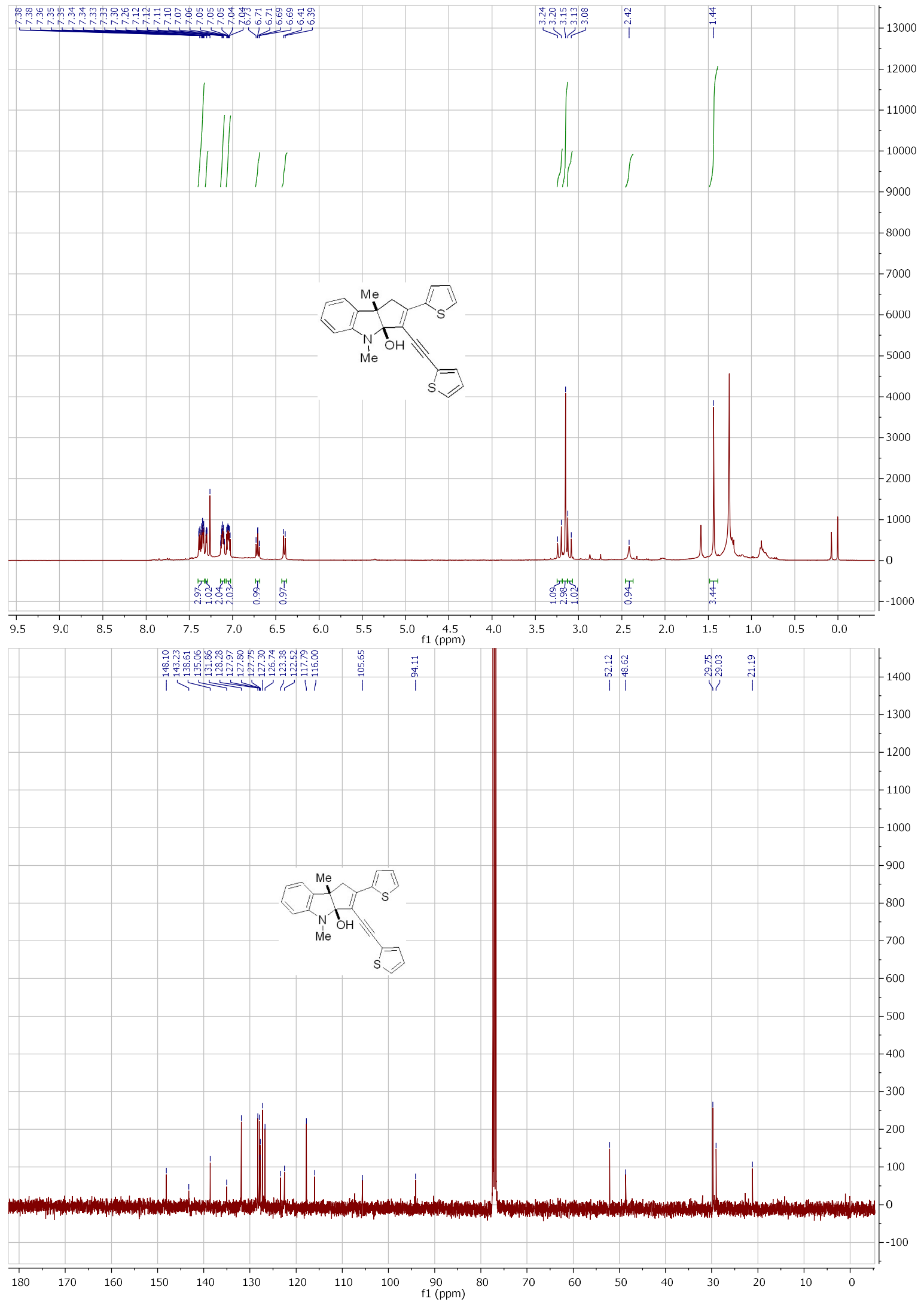




\section{$3 a o$}
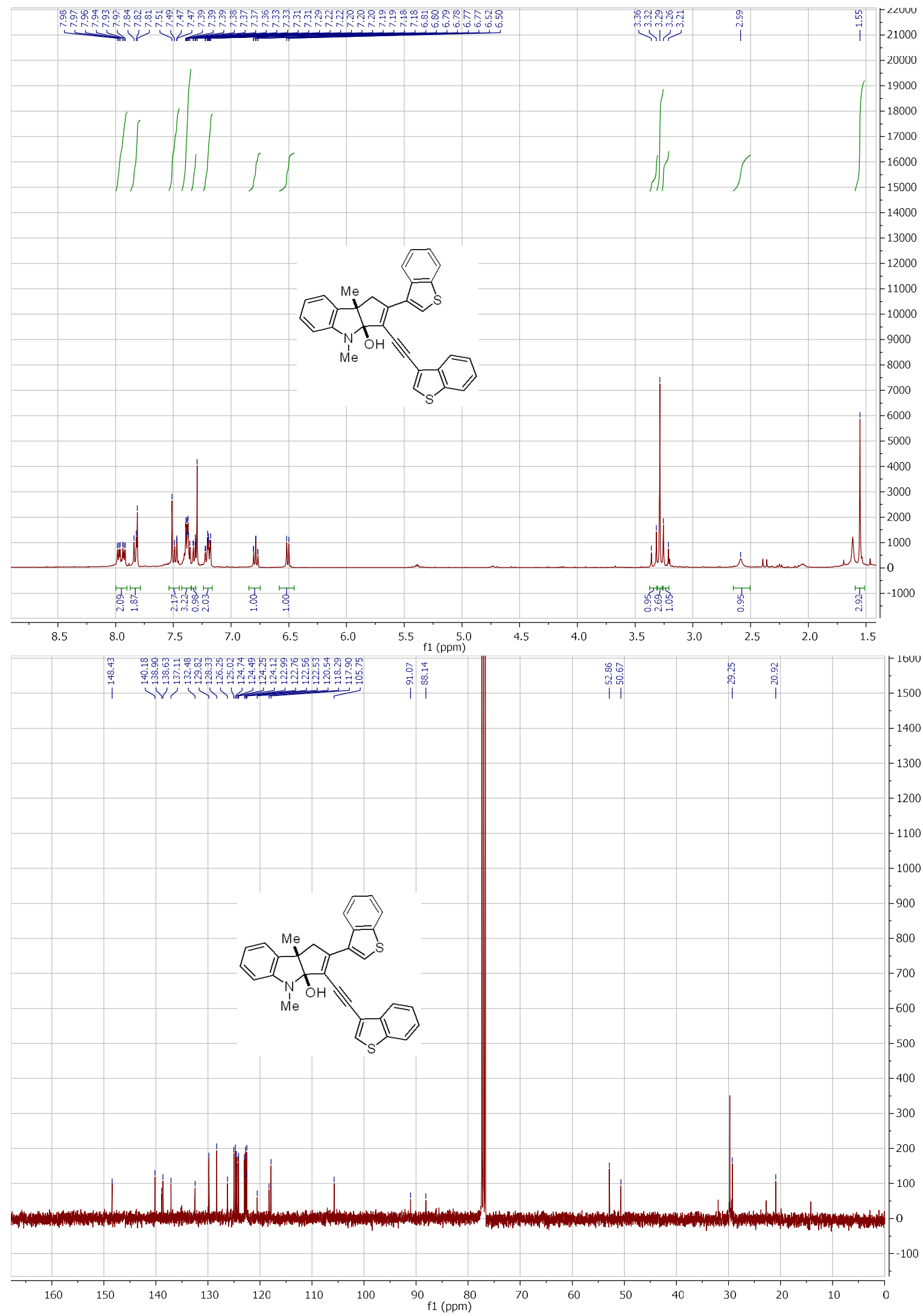


\section{$8 a a$}
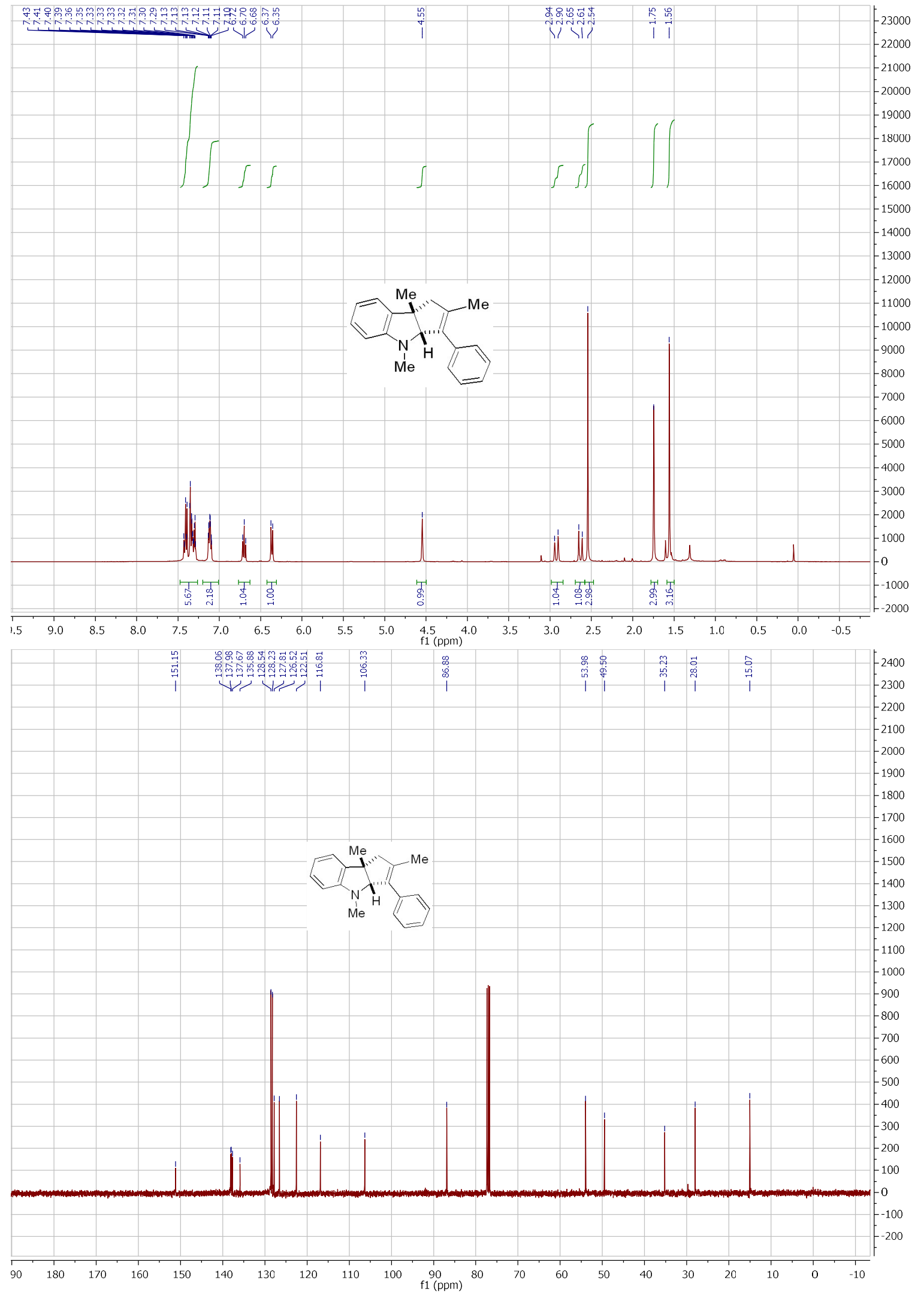


\section{$8 b a$}
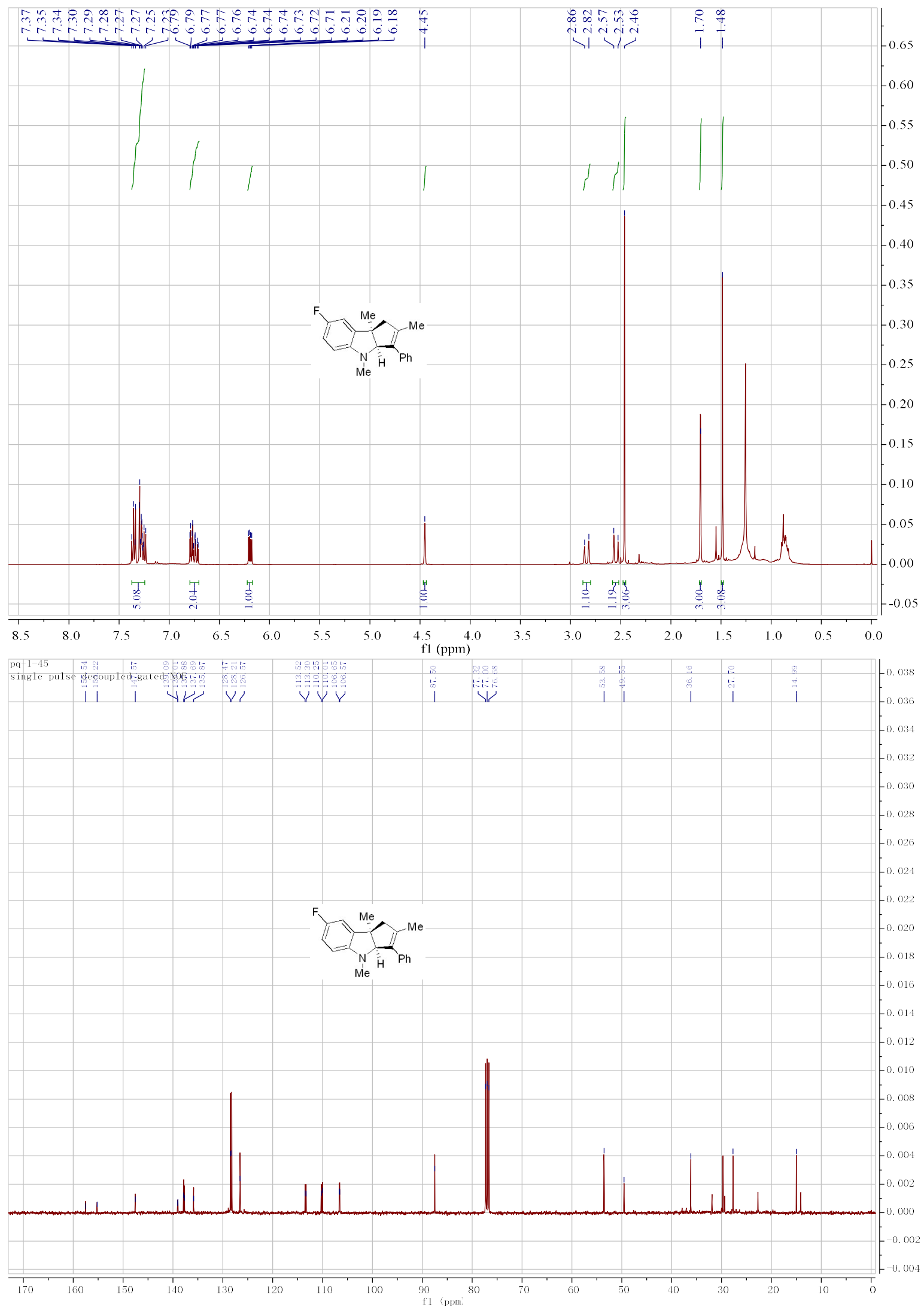


\section{$8 \mathrm{ca}$}

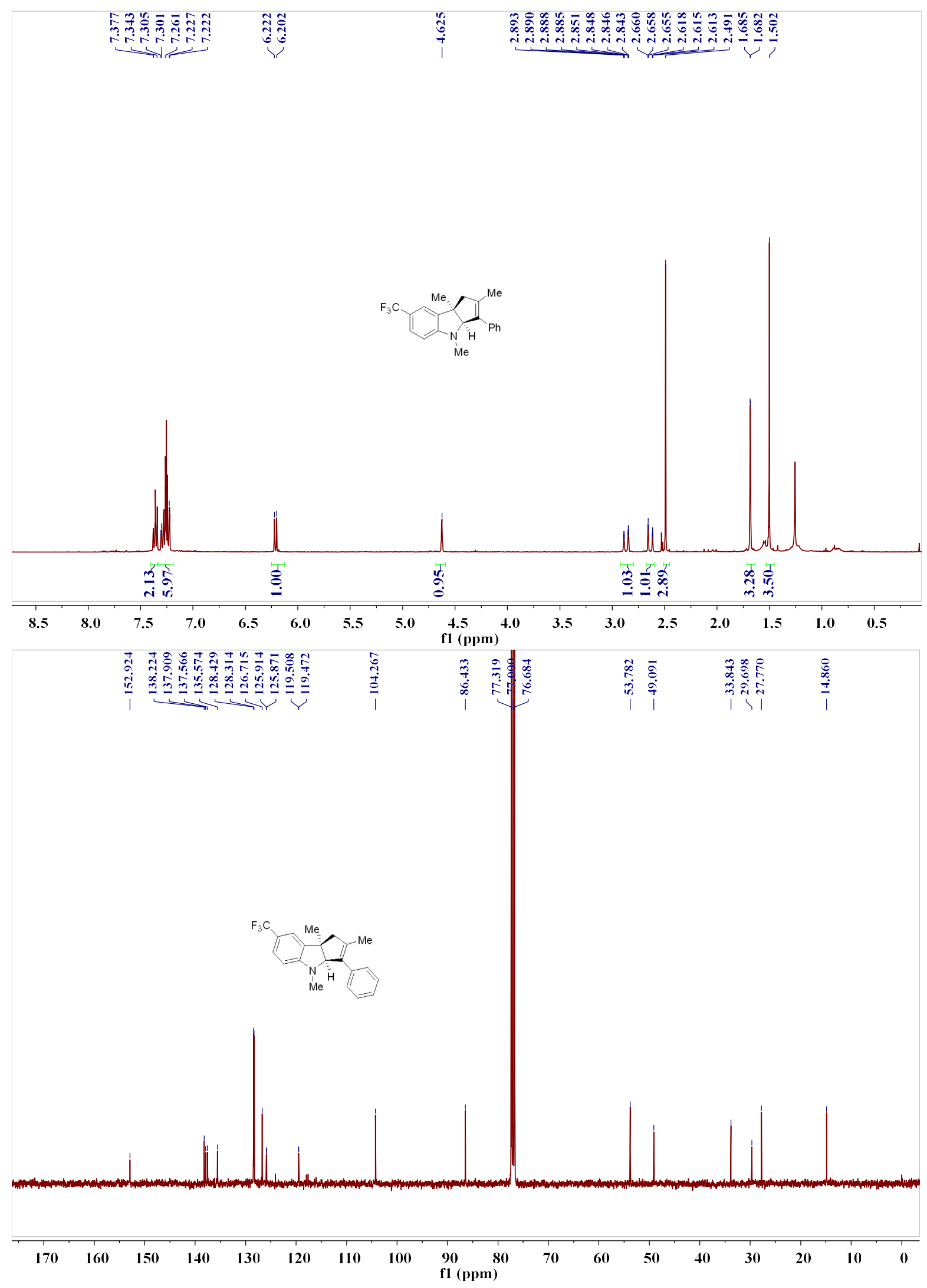




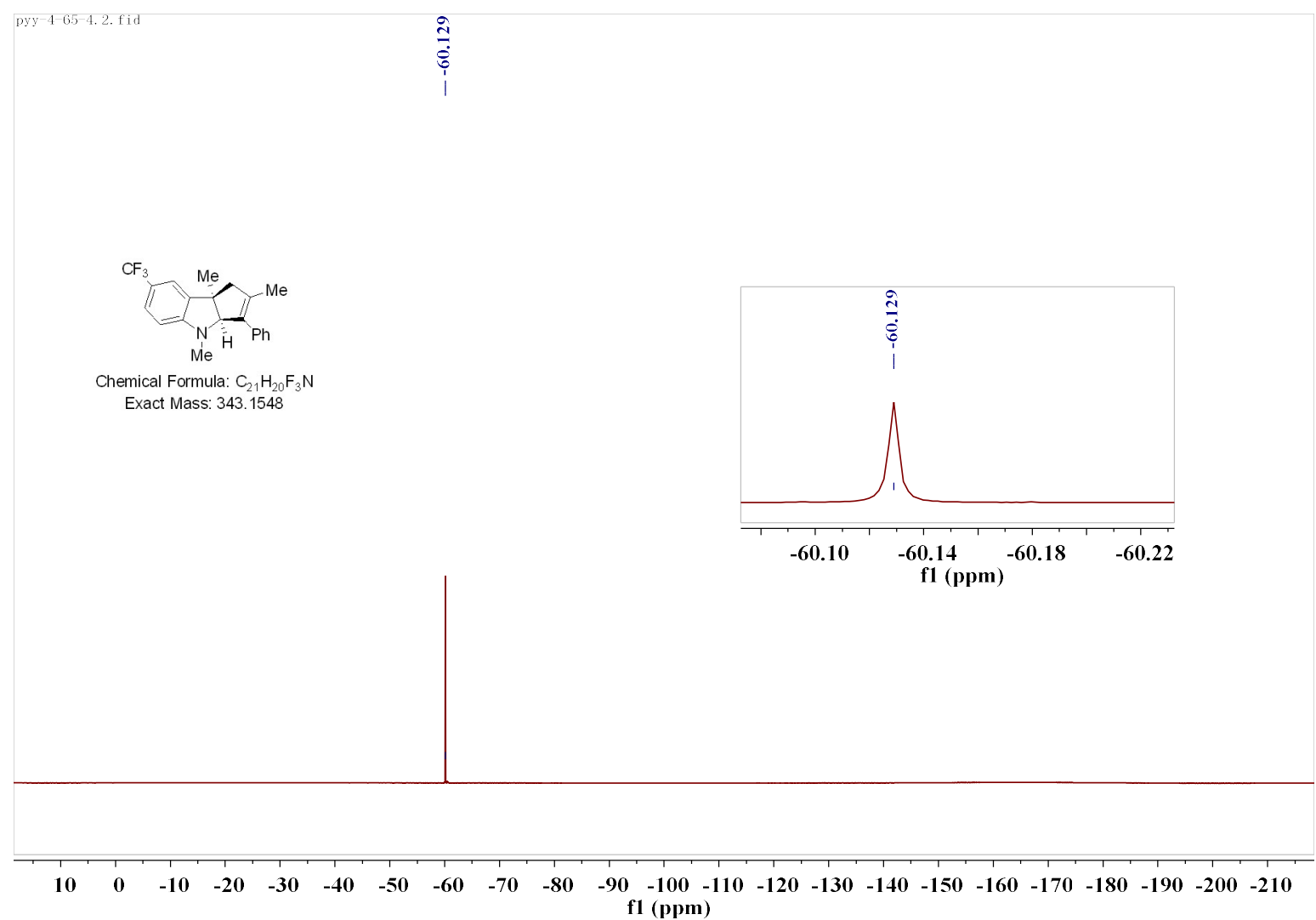




\section{8da}
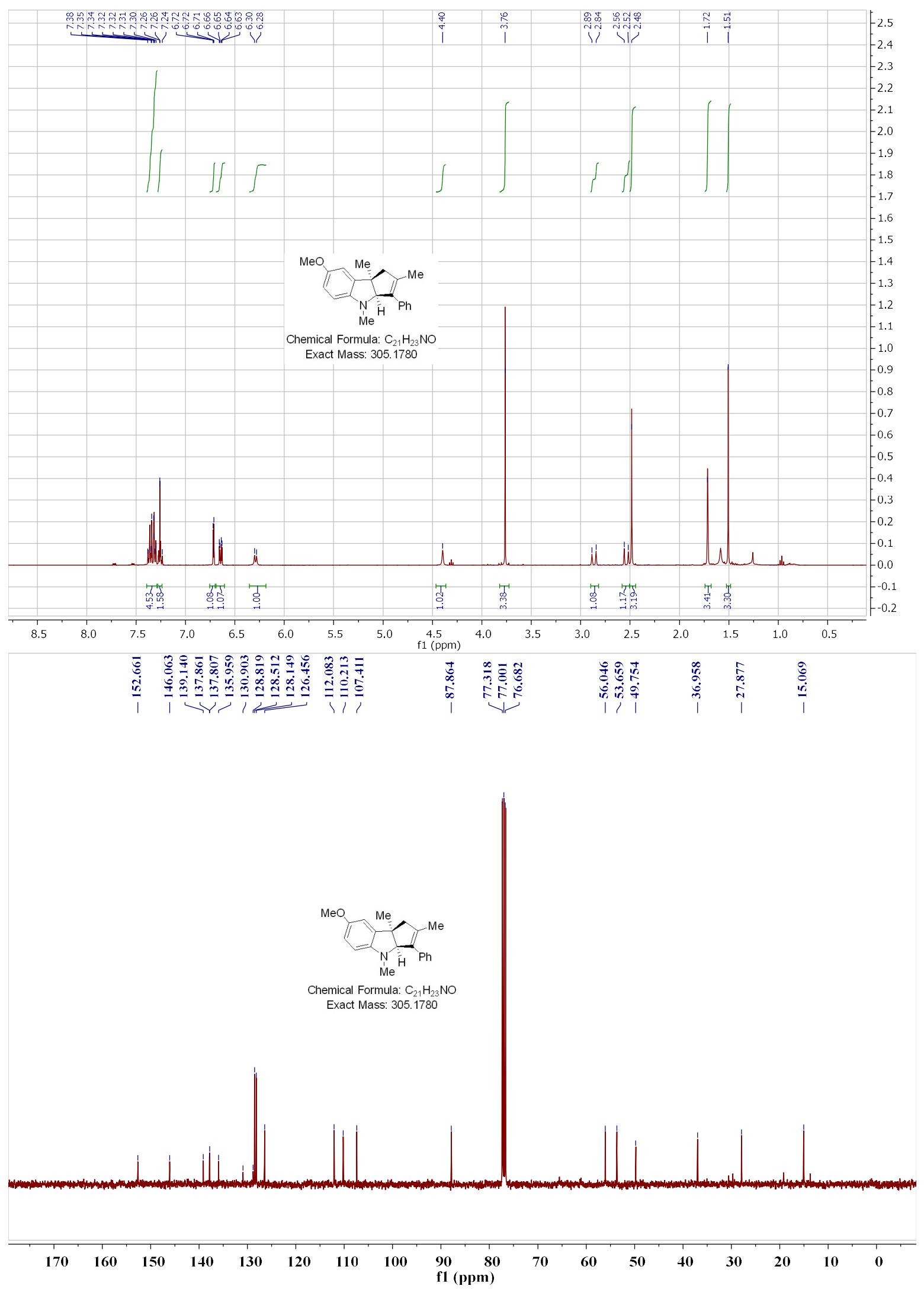


\section{8ea}

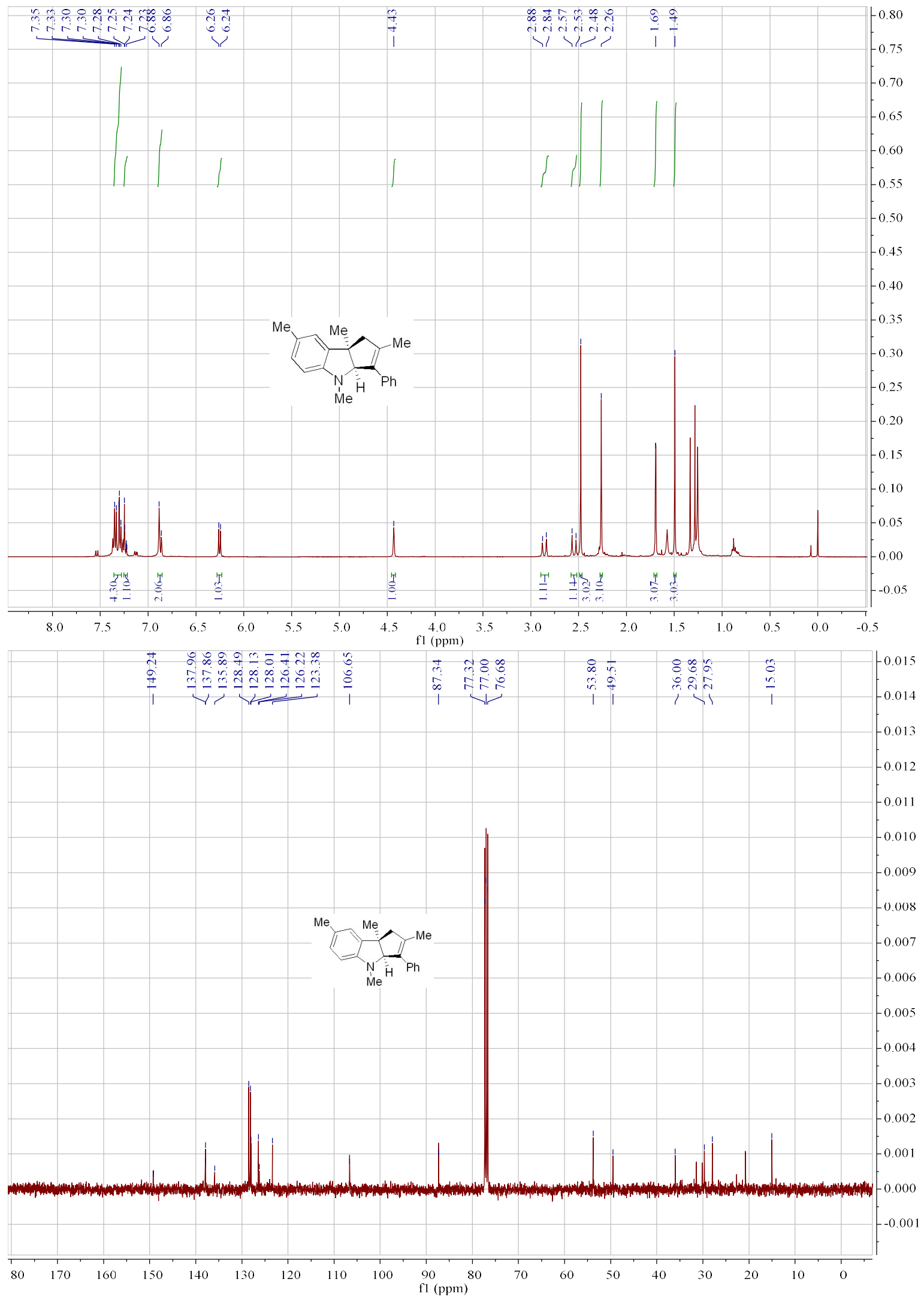


$8 f a$

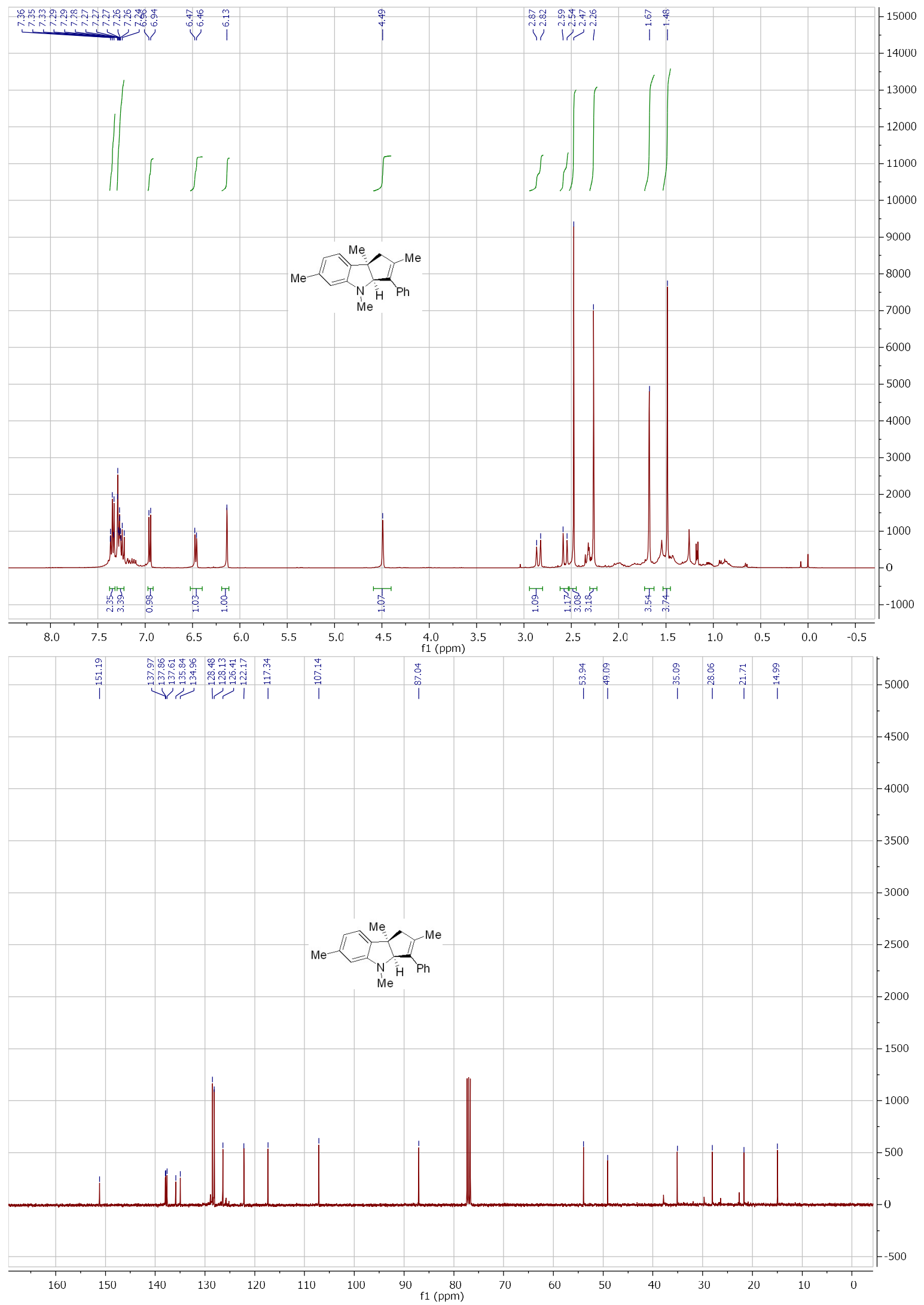




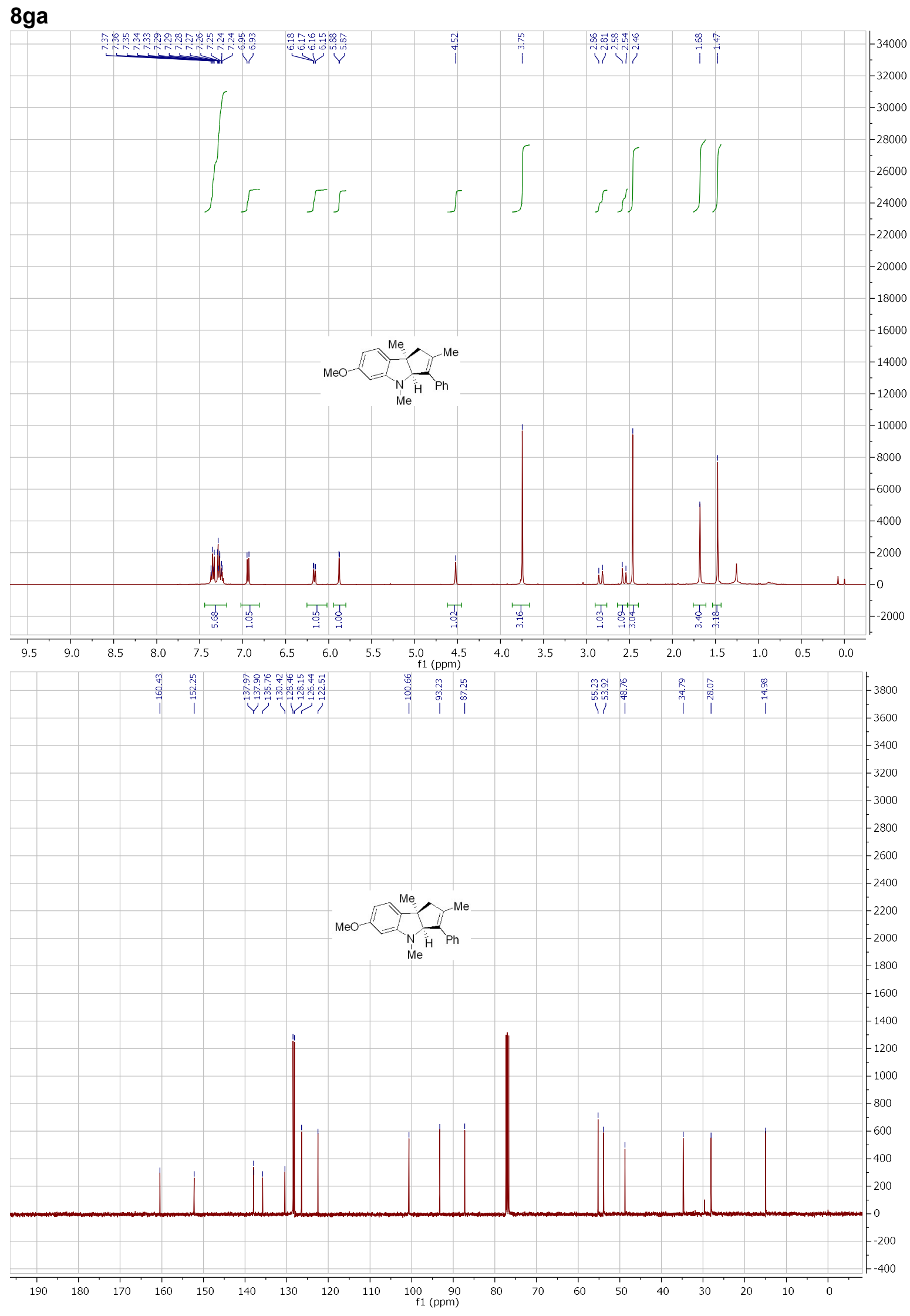




\section{8ha}

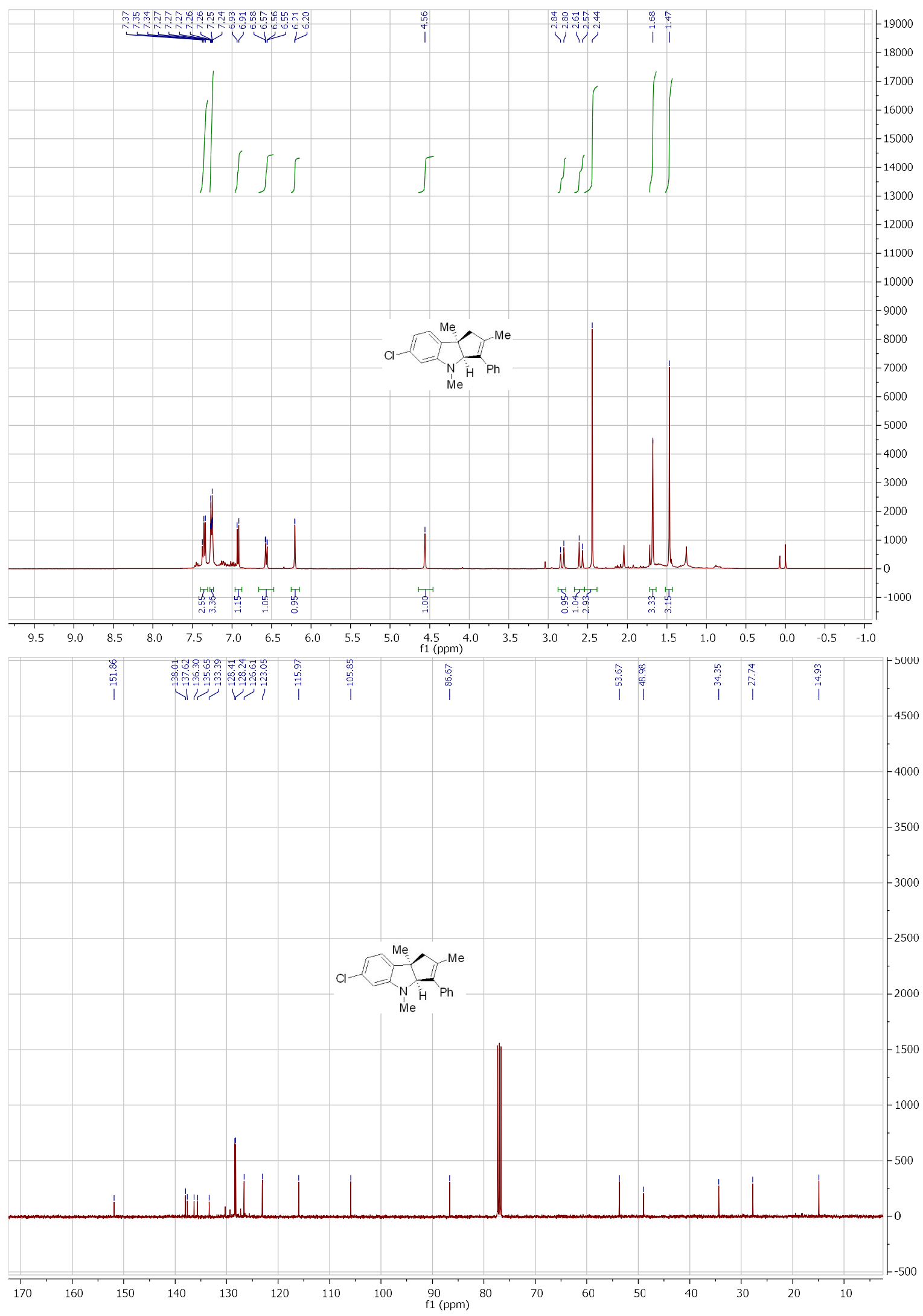




\section{8ja}
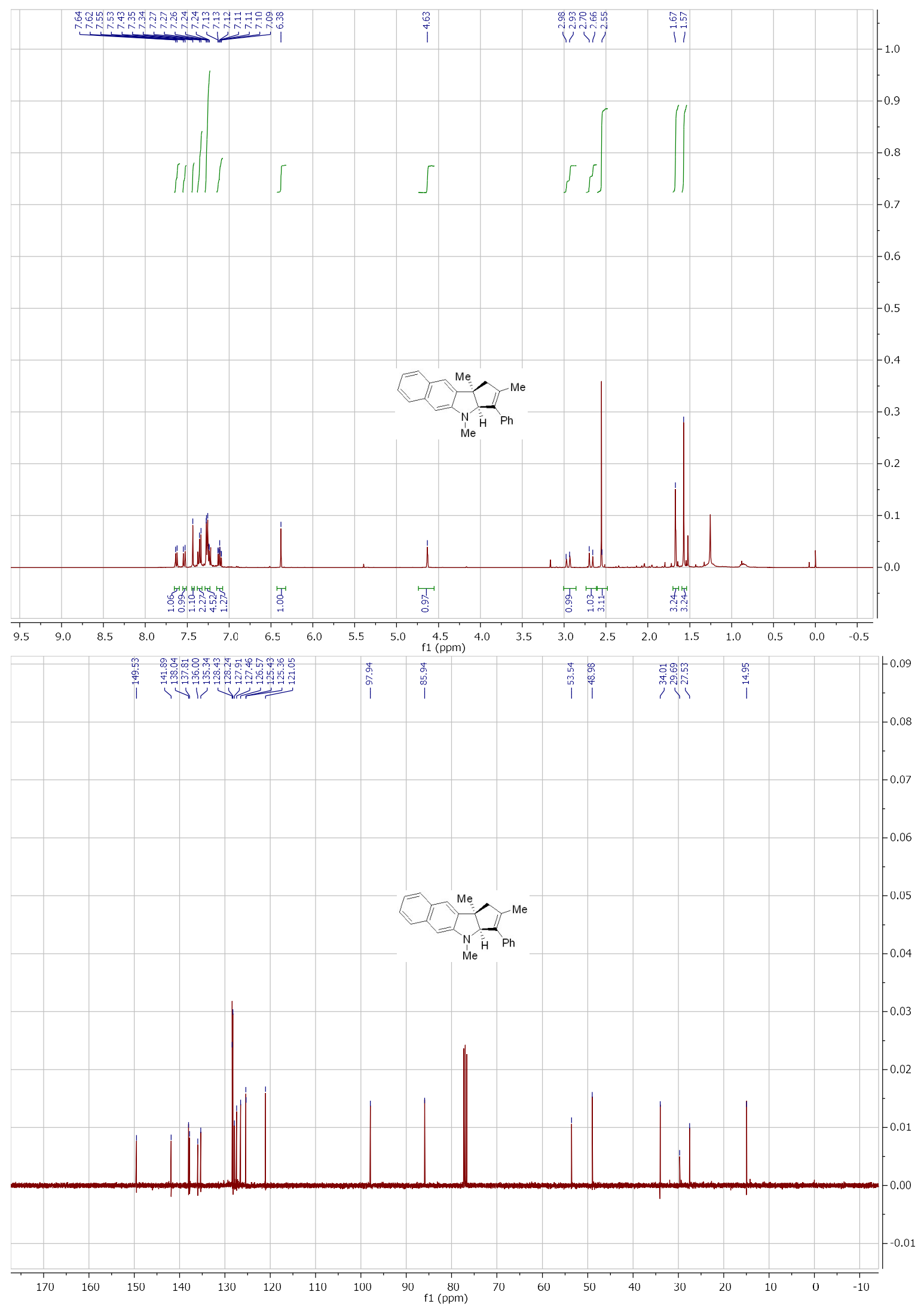
$81 a$
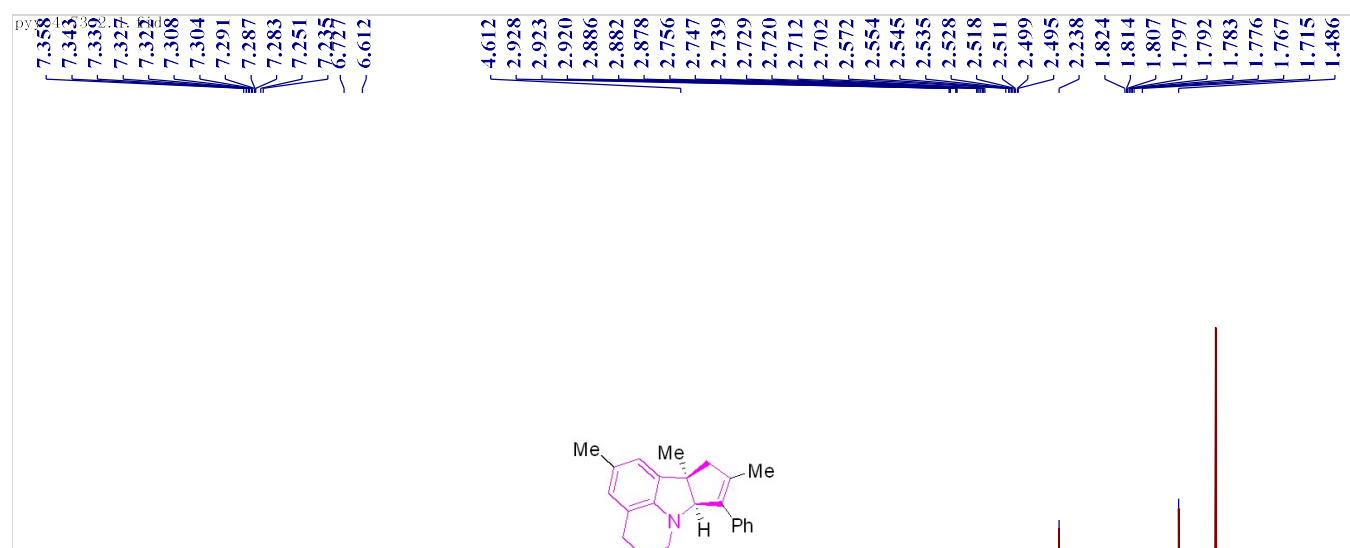

Chemical Formula: $\mathrm{C}_{23} \mathrm{H}_{25} \mathrm{~N}$ Exact Mass: 315.1987
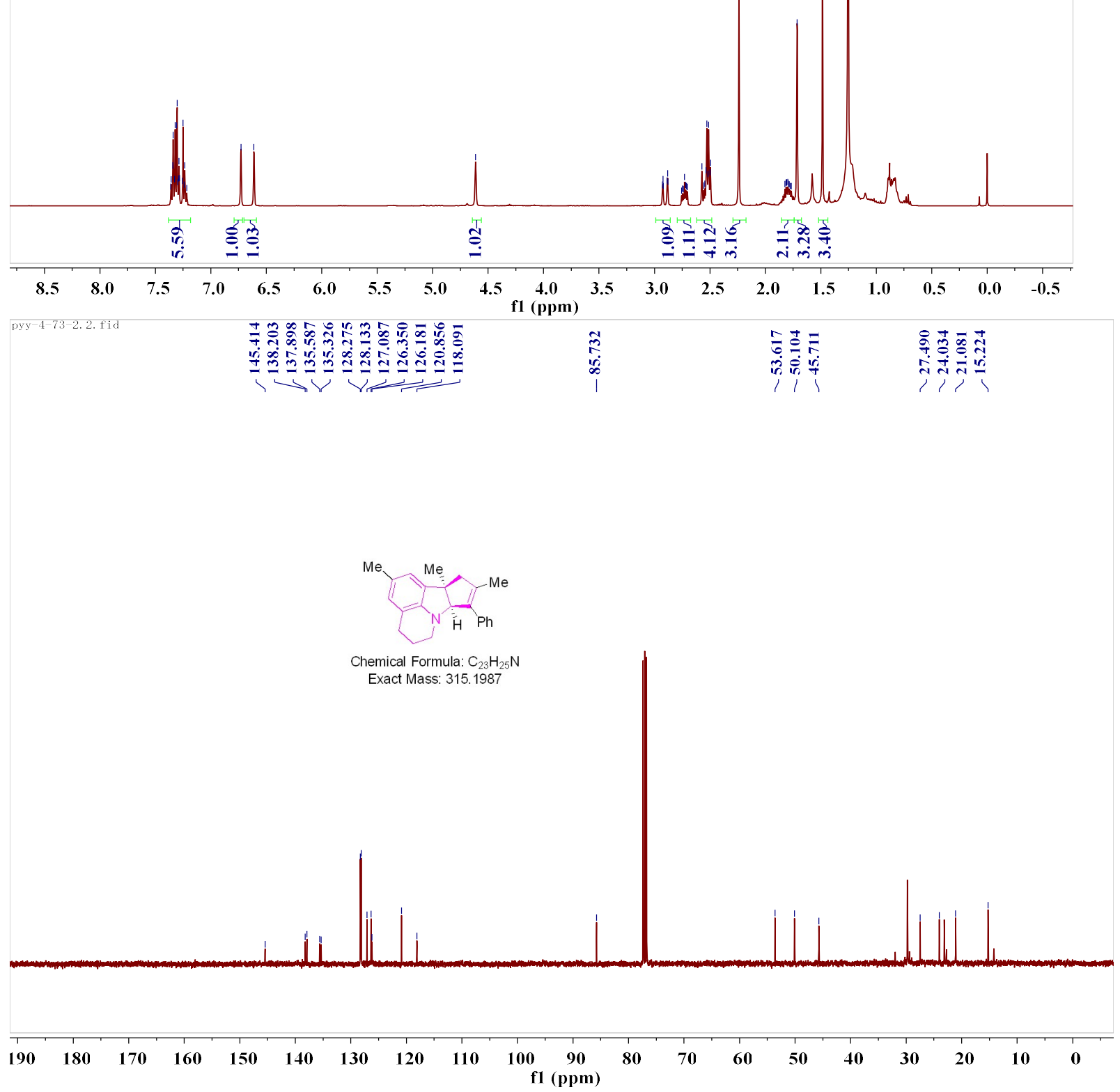


\section{8ma}

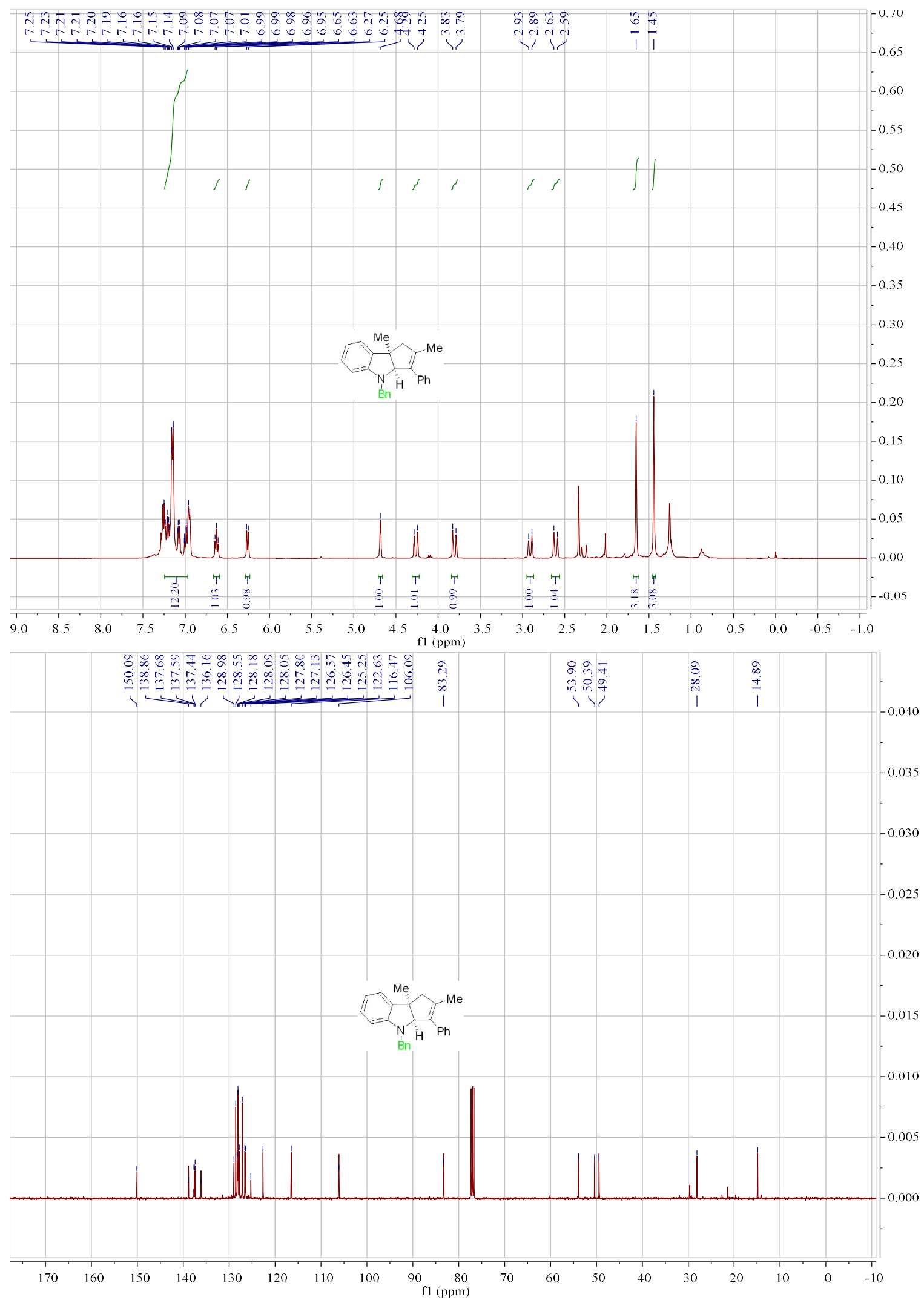




\section{$80 a$}
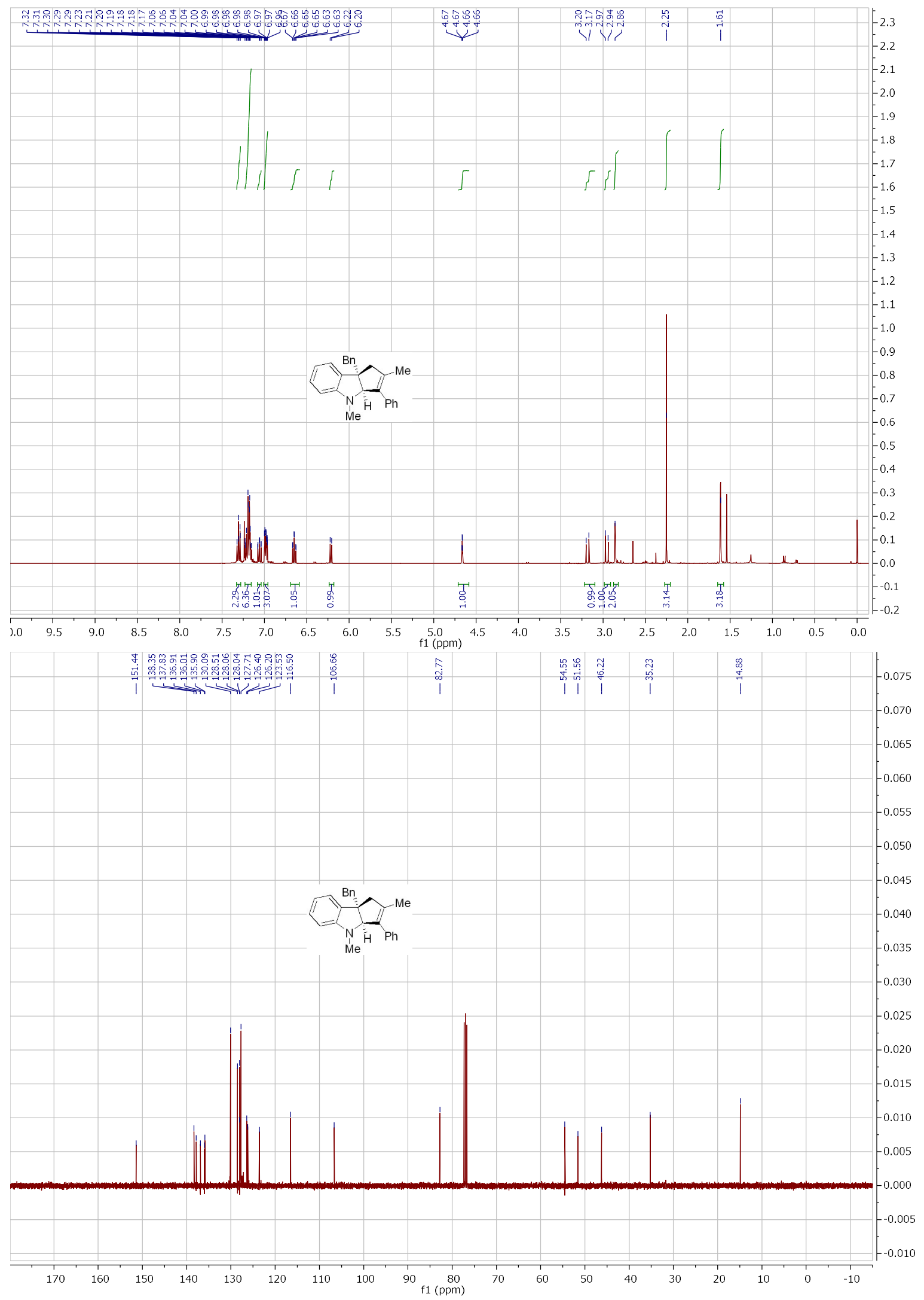
8pa
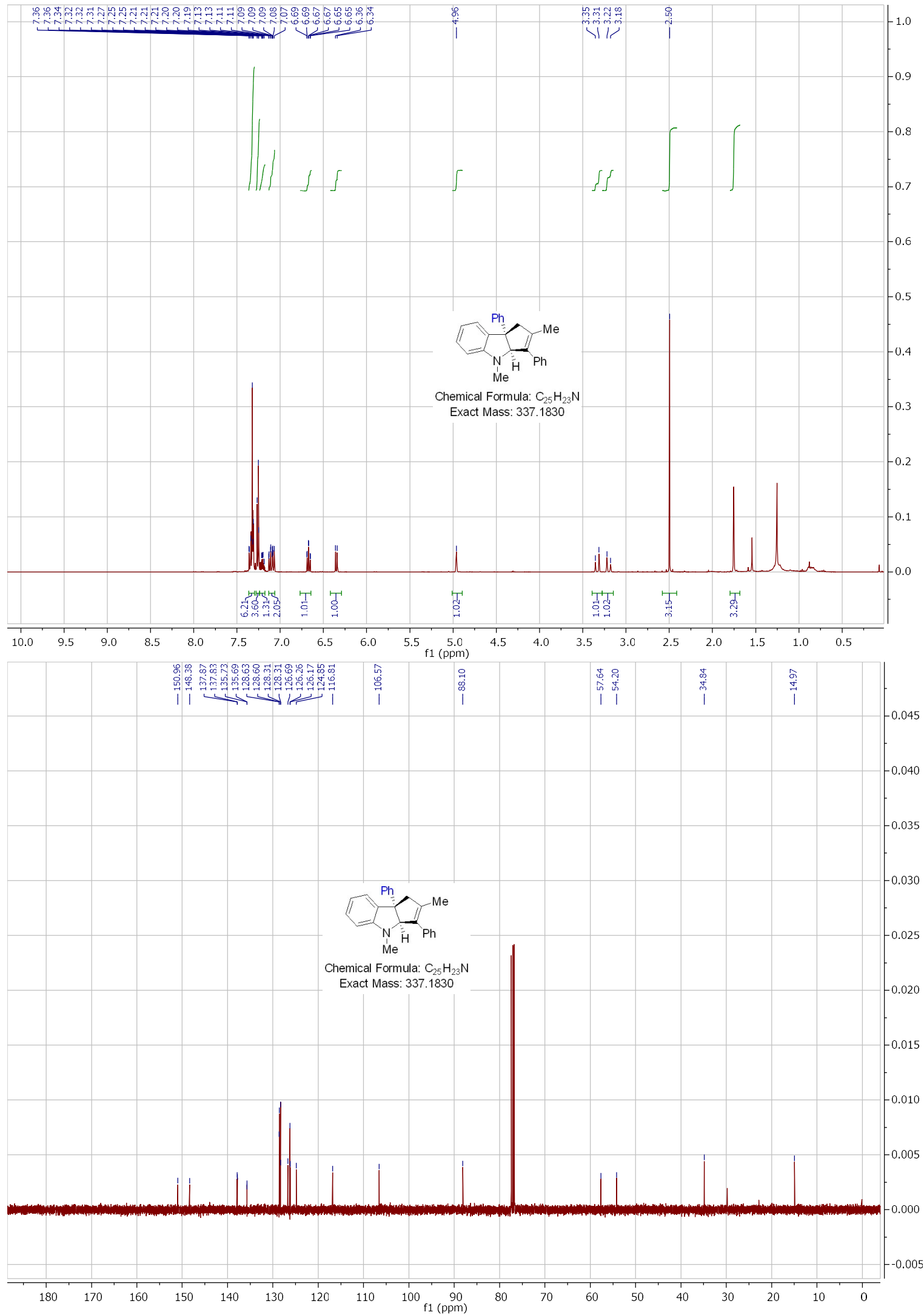


\section{$8 q a$}
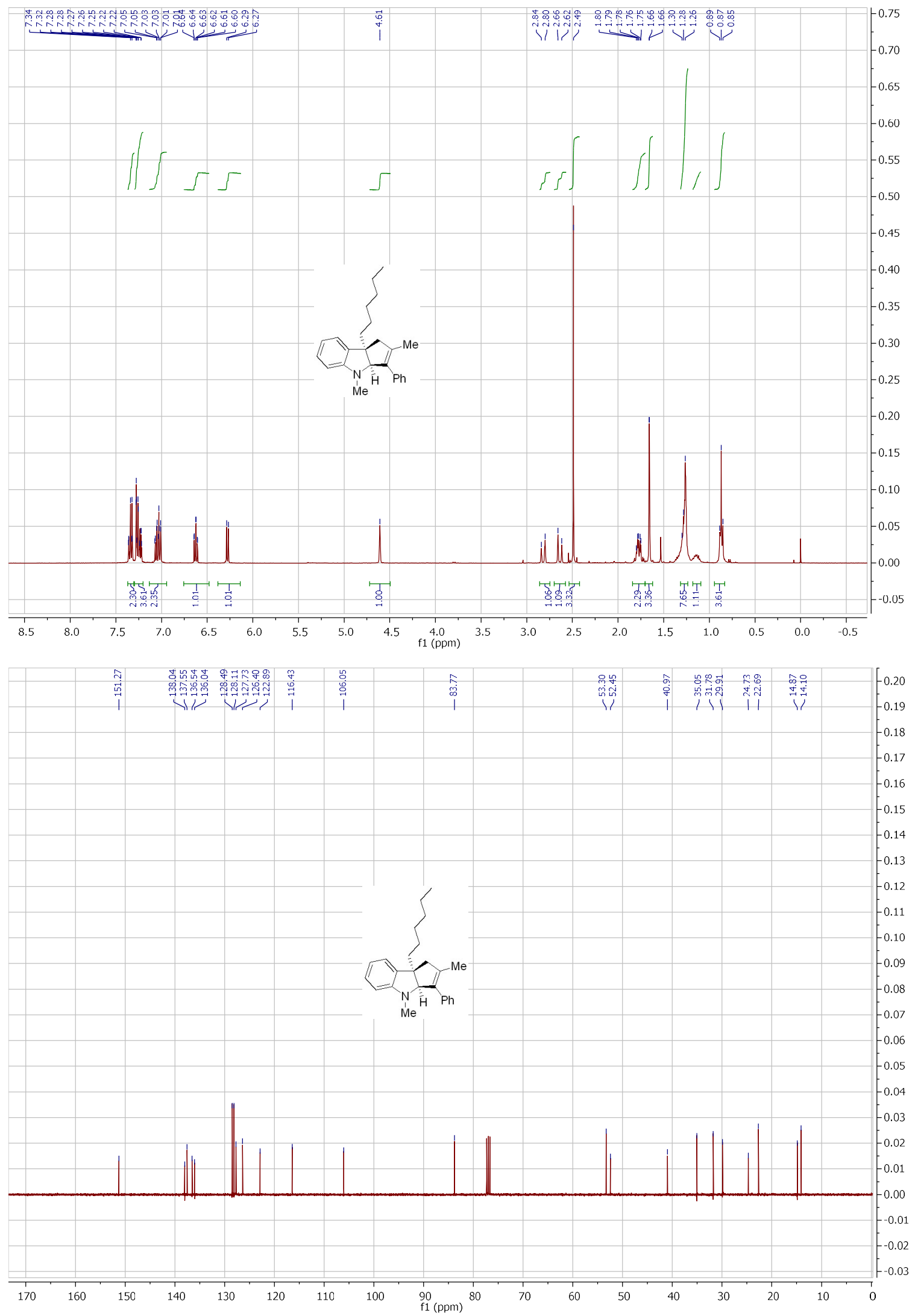


\section{$8 a b$}

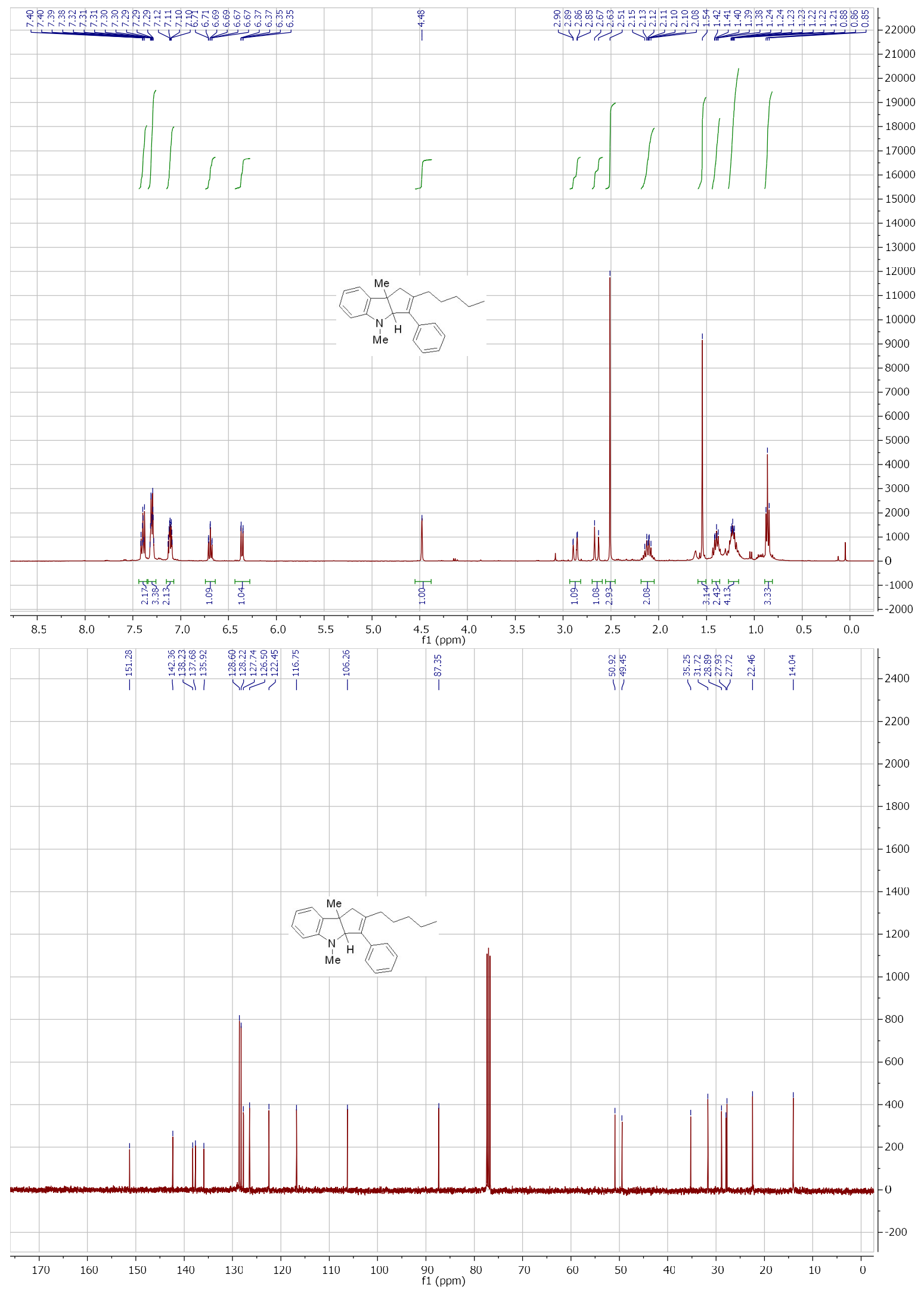




\section{$8 a c$}

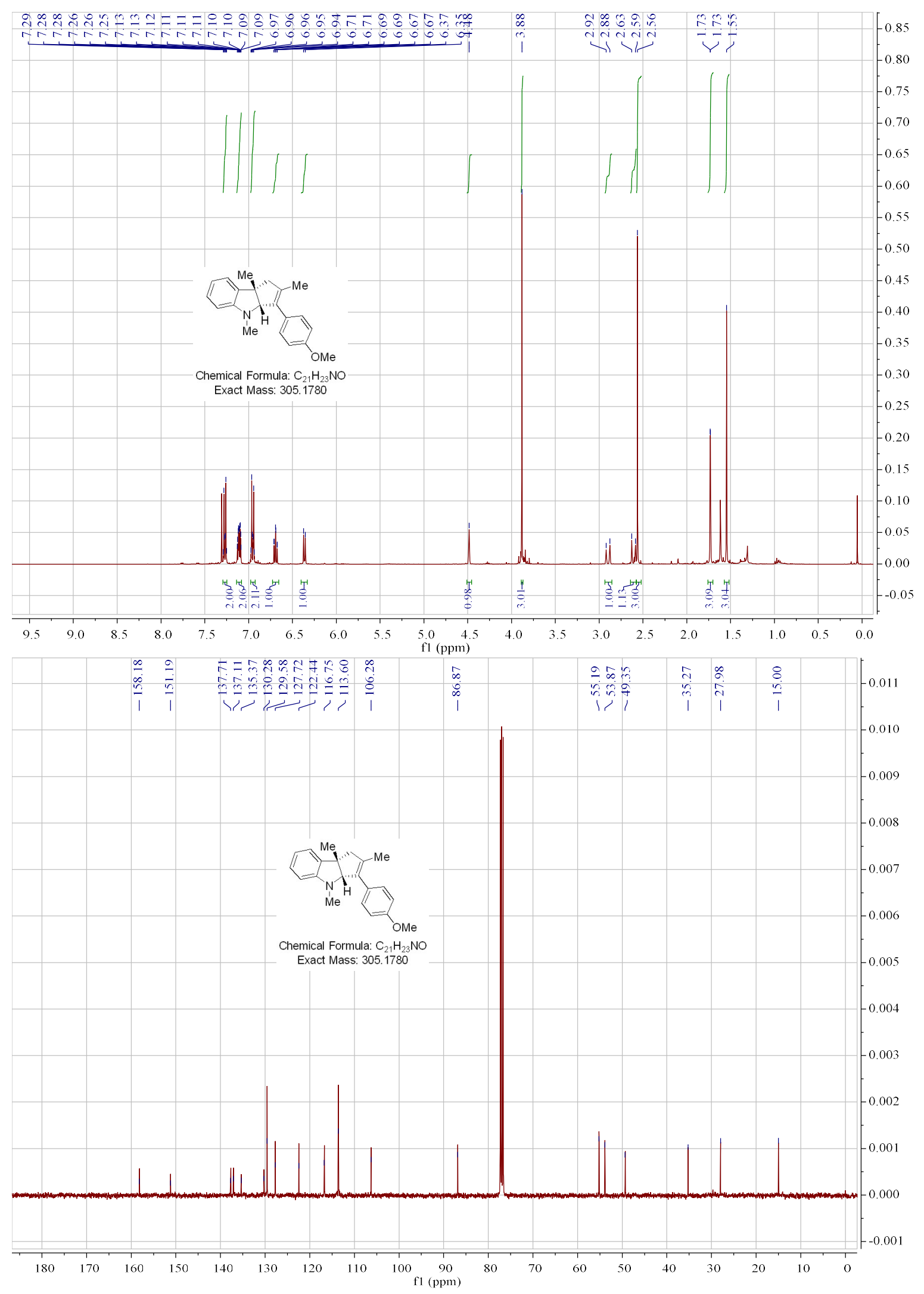




\section{8ad}
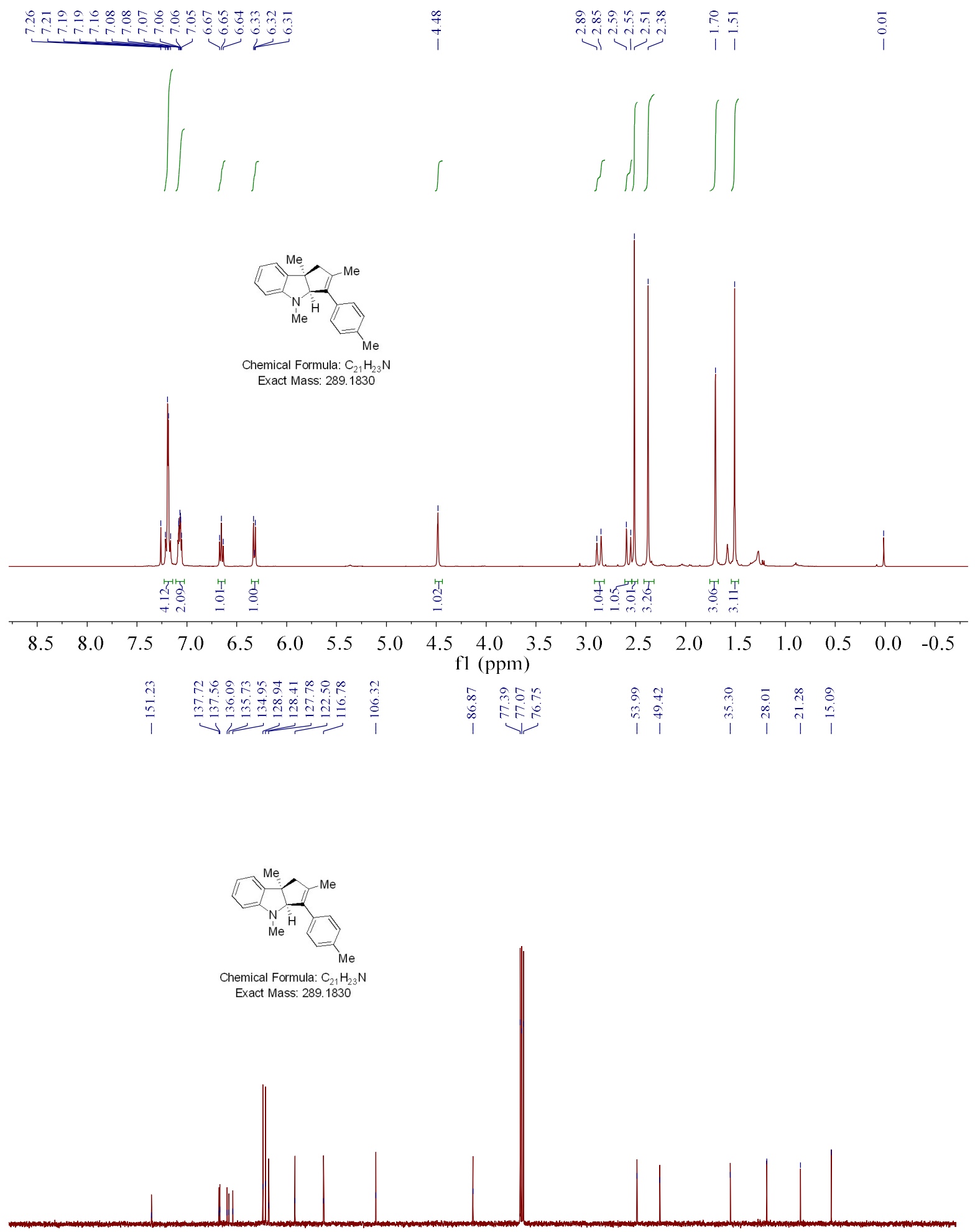

$\begin{array}{llllllllllllllllll}170 & 160 & 150 & 140 & 130 & 120 & 110 & 100 & \begin{array}{l}90 \\ \mathrm{fl}(\mathrm{ppm})\end{array} & \begin{array}{ll}80 \\ 70\end{array} & 60 & 50 & 40 & 30 & 20 & 10 & 0\end{array}$ 


\section{$8 a e$}
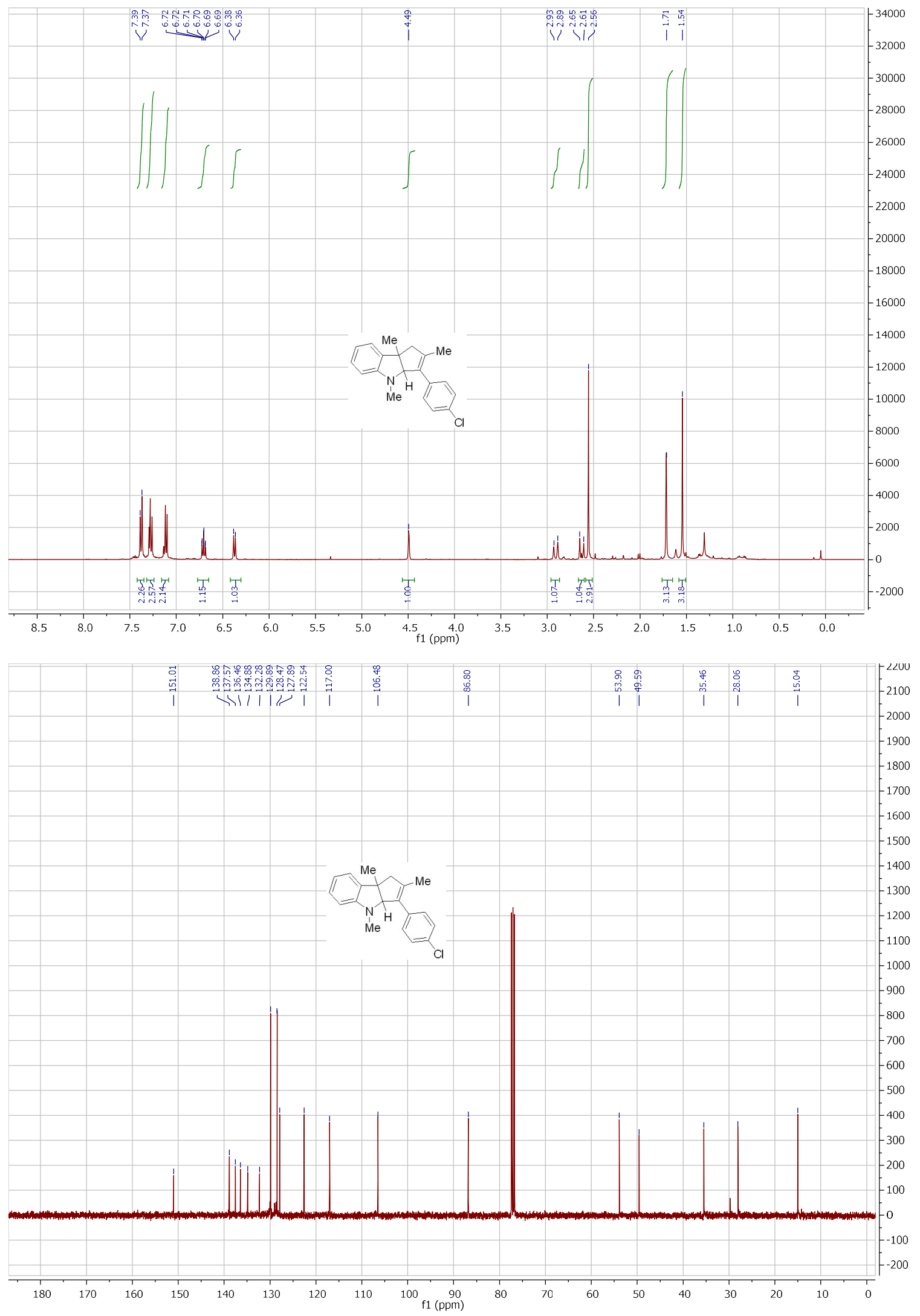


\section{8af}
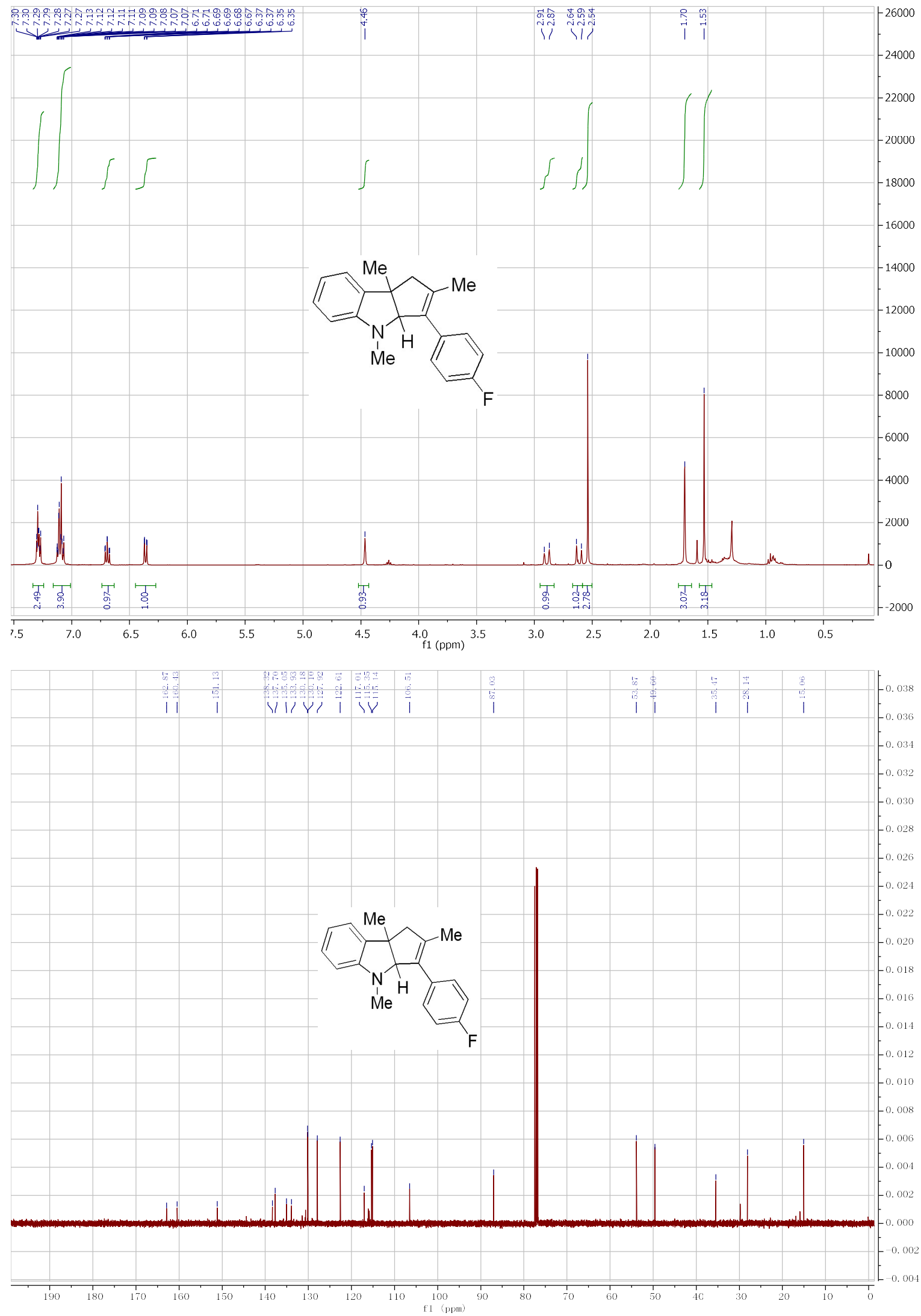


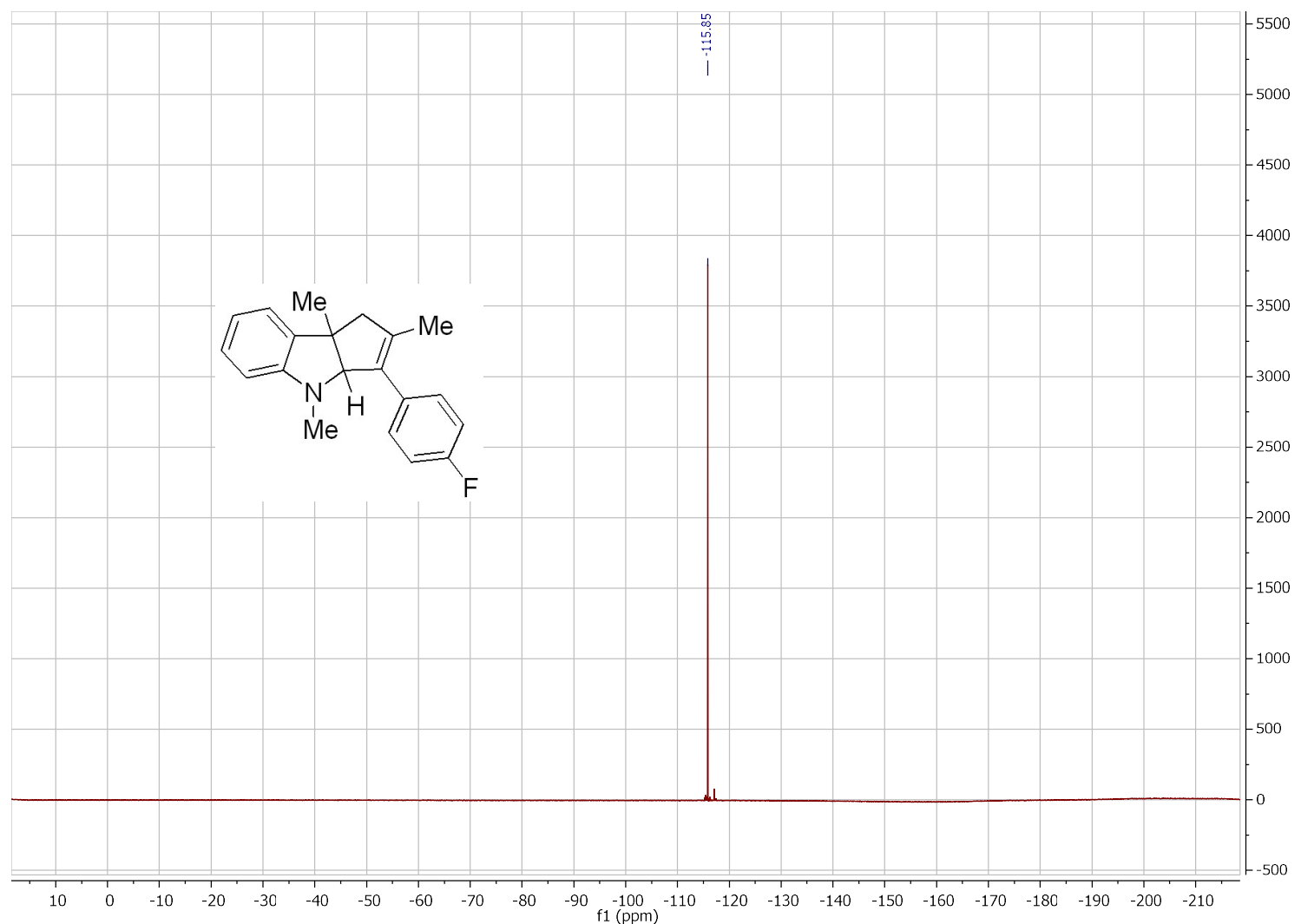


$8 a g$
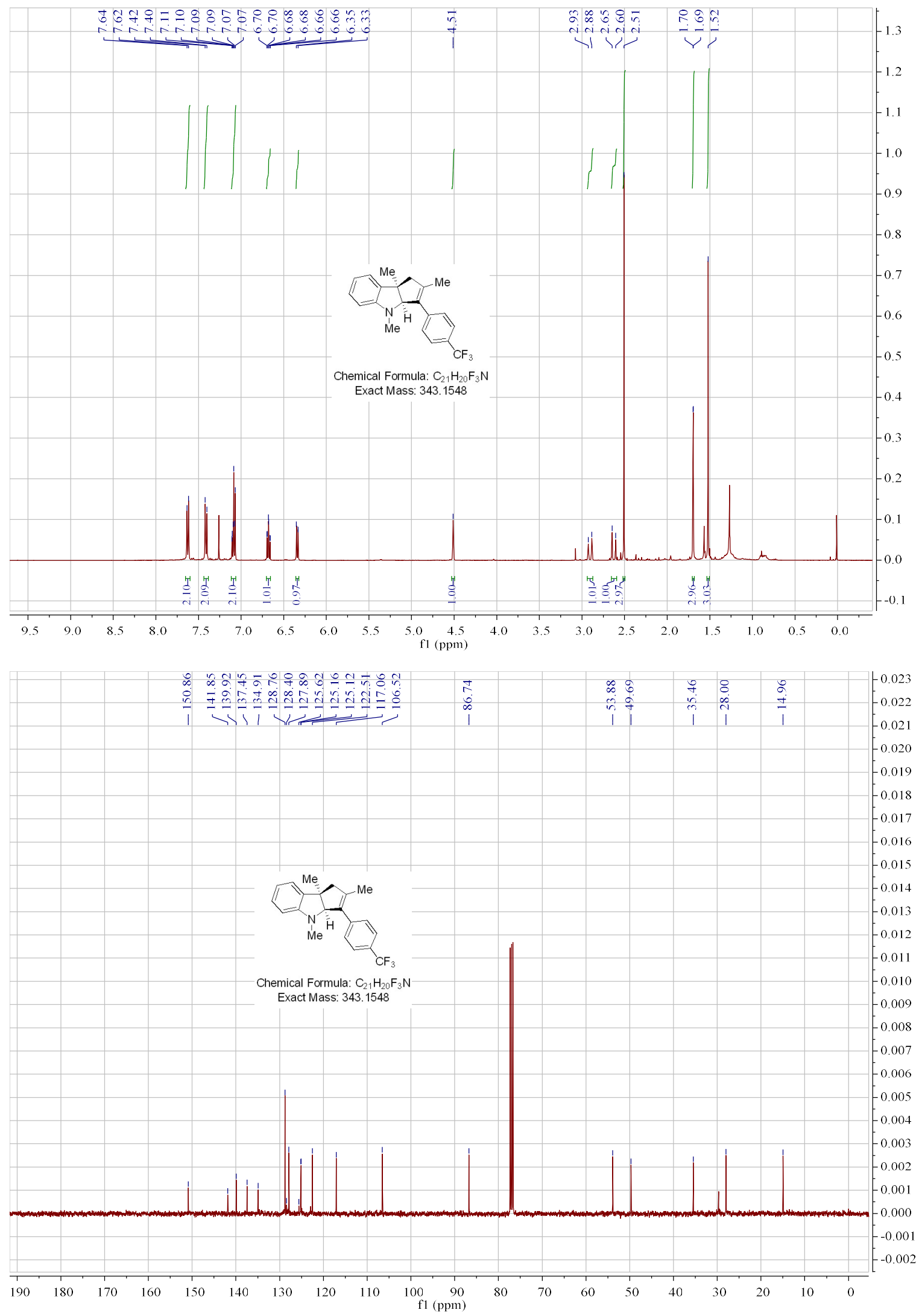


\section{8ah}

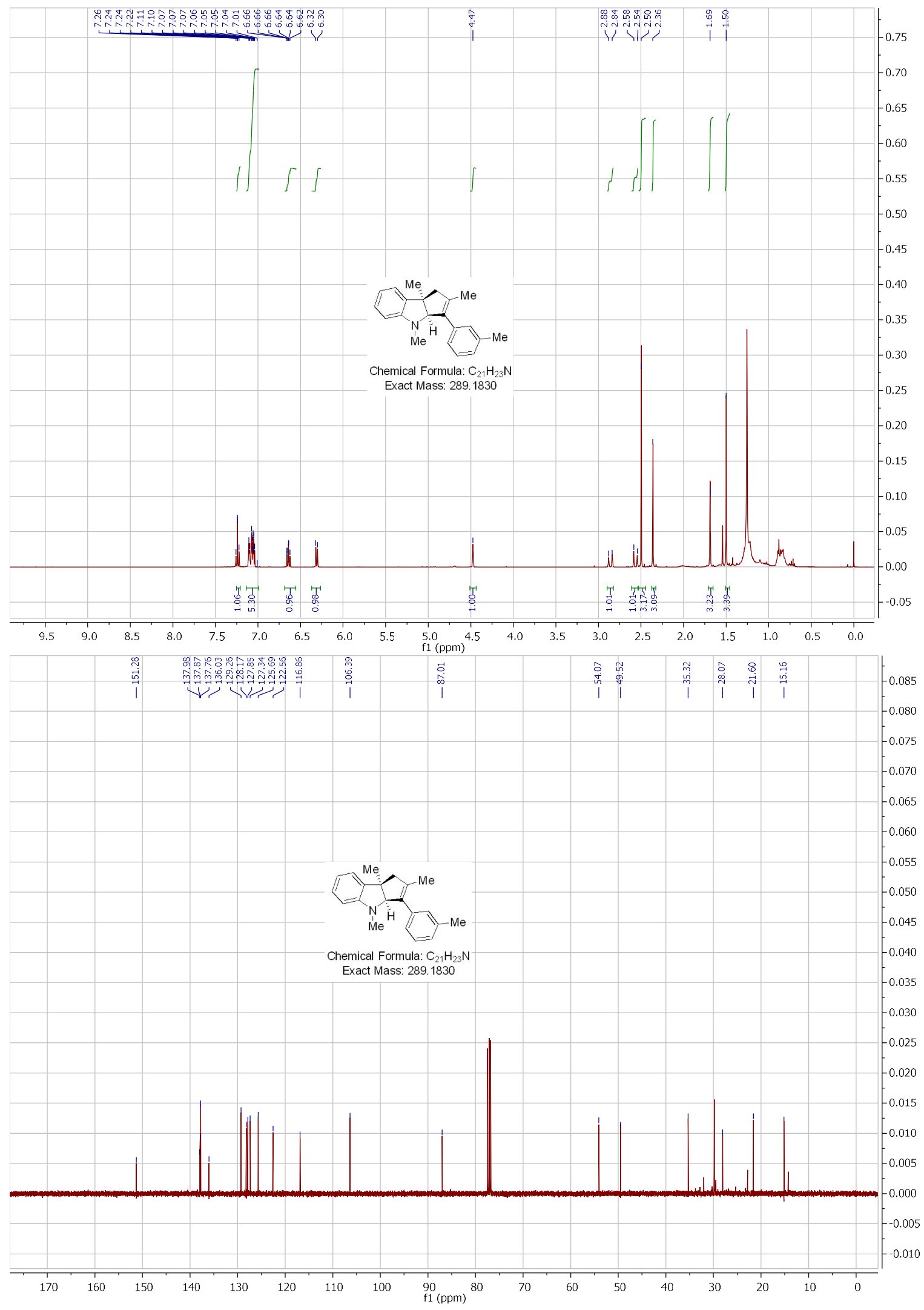




\section{8ai}
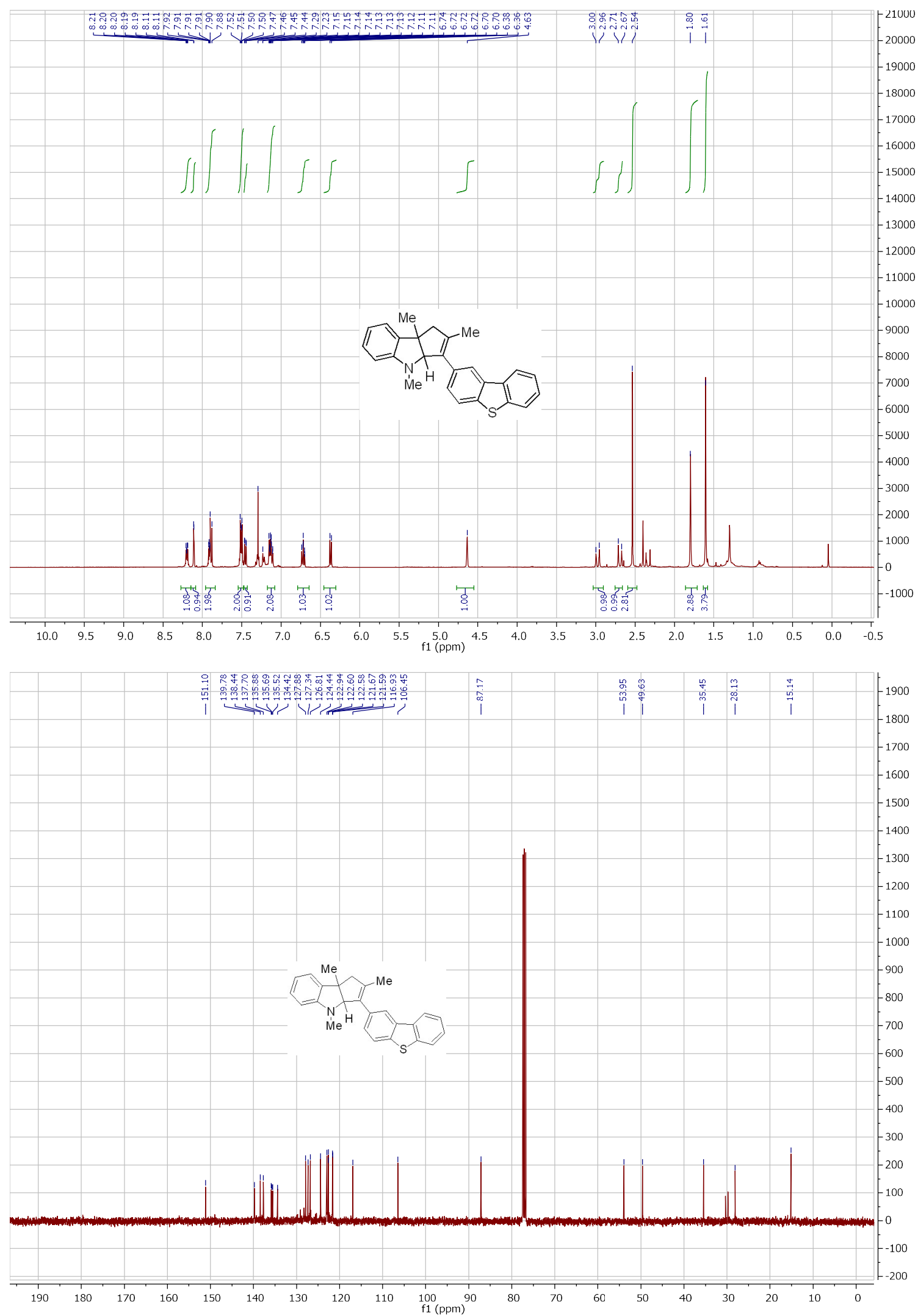
8aj

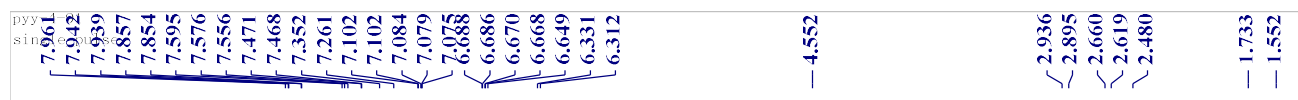
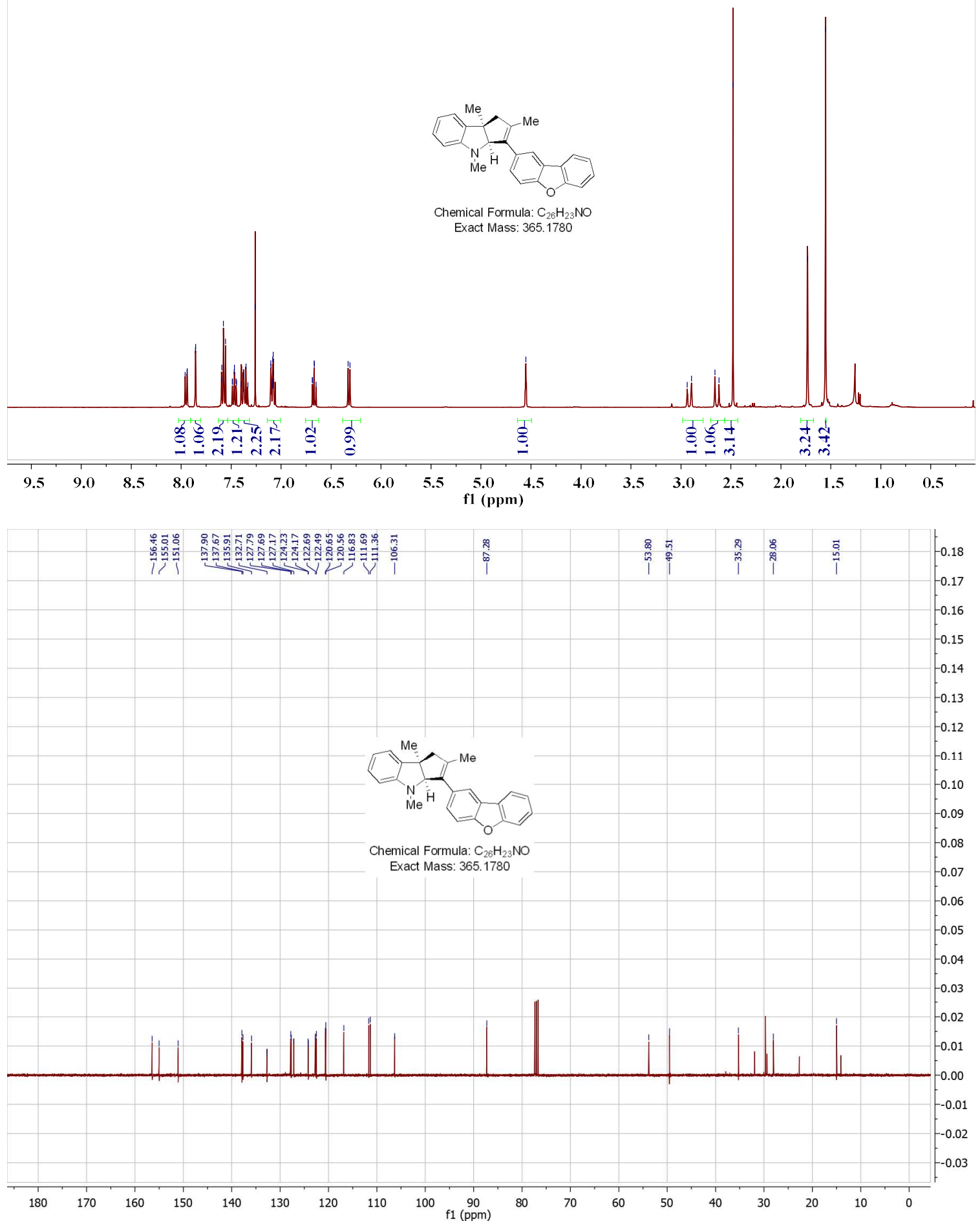


\section{8ak}

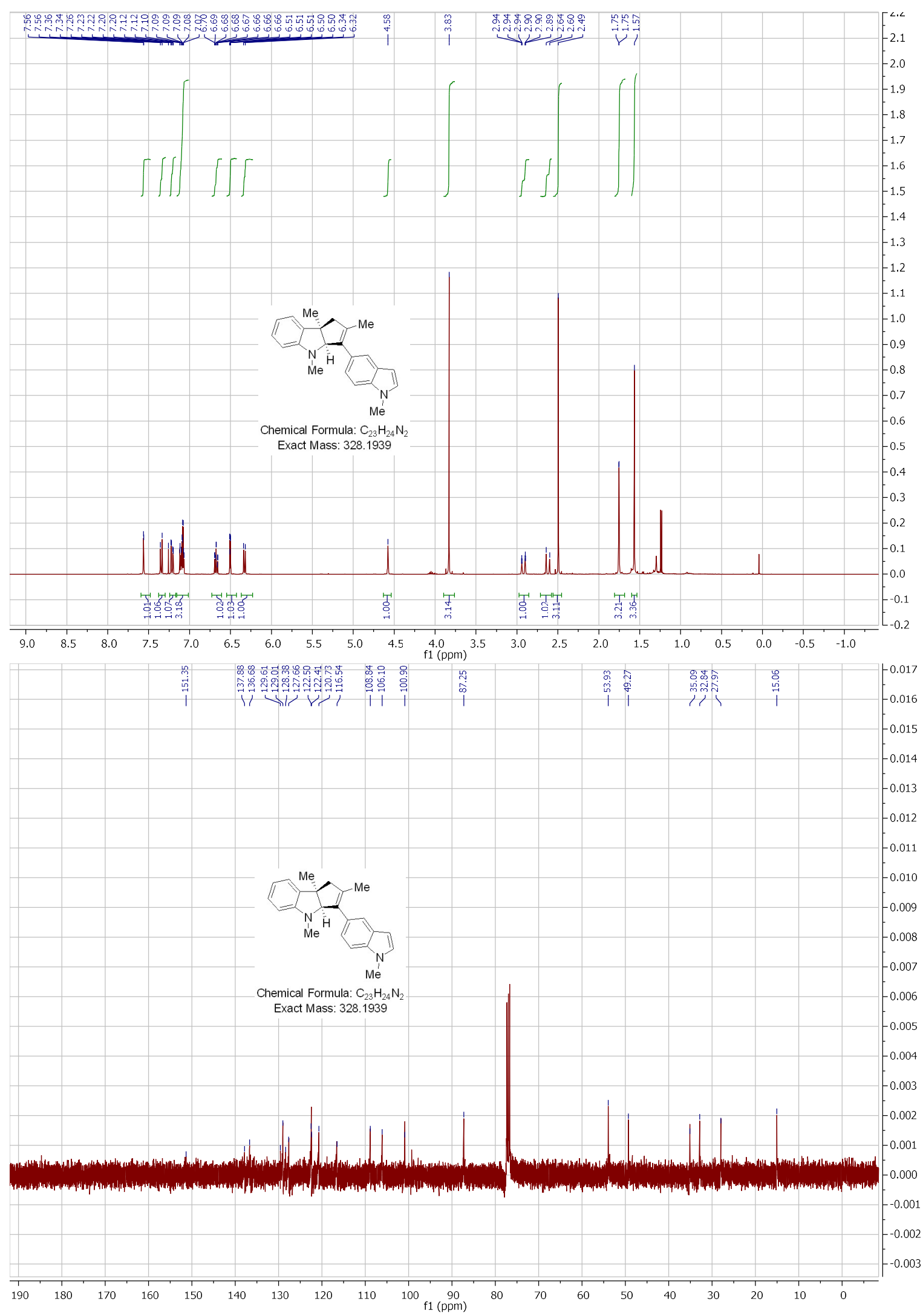




\section{8al}

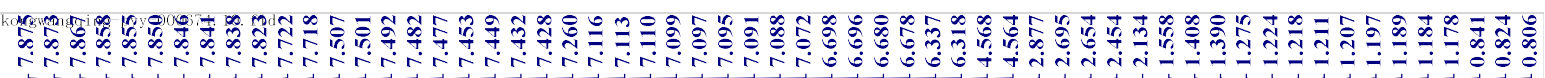

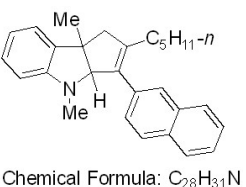

Exact Mass: 381.2457
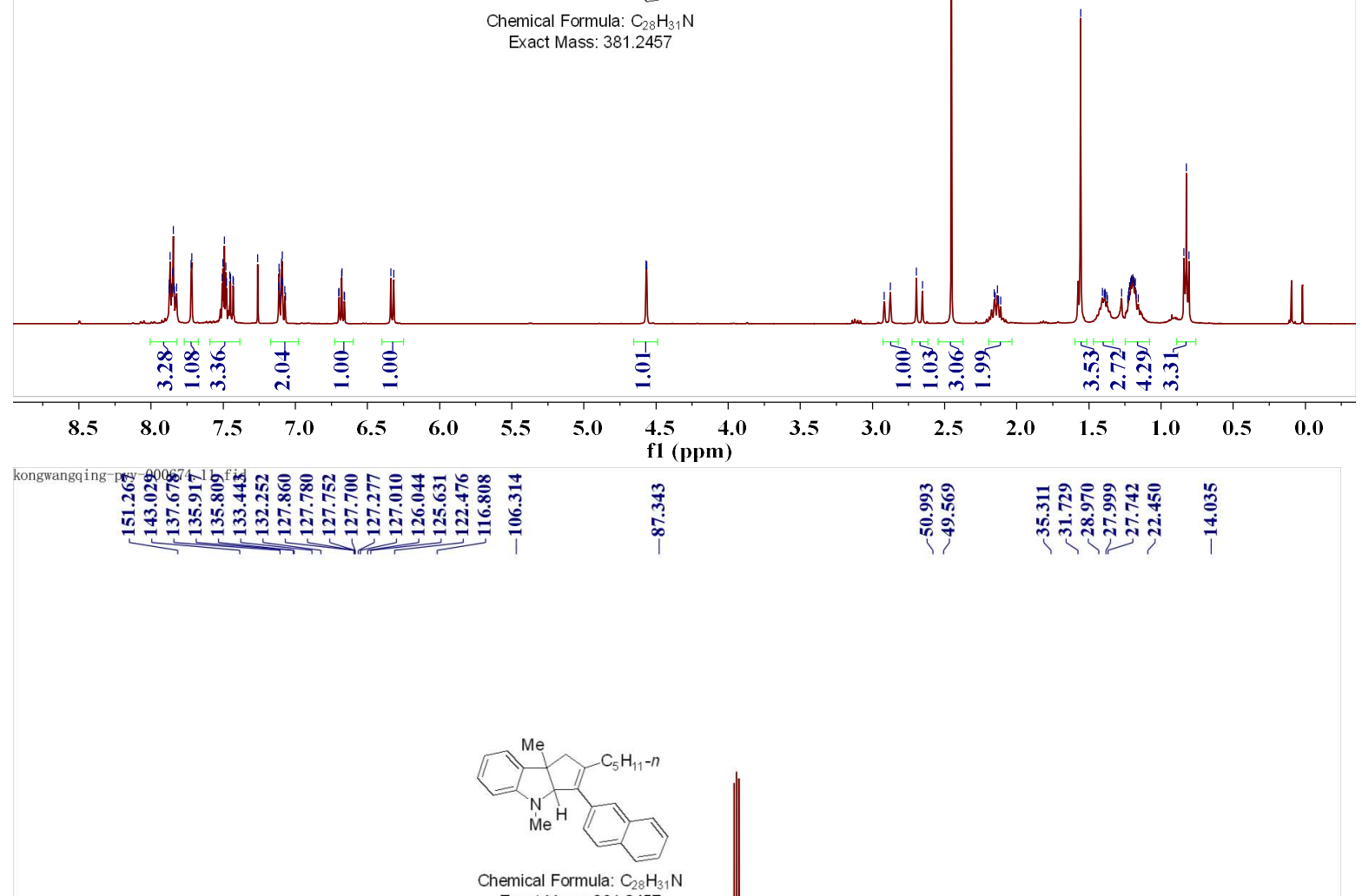

Exact Mass: 381.2457
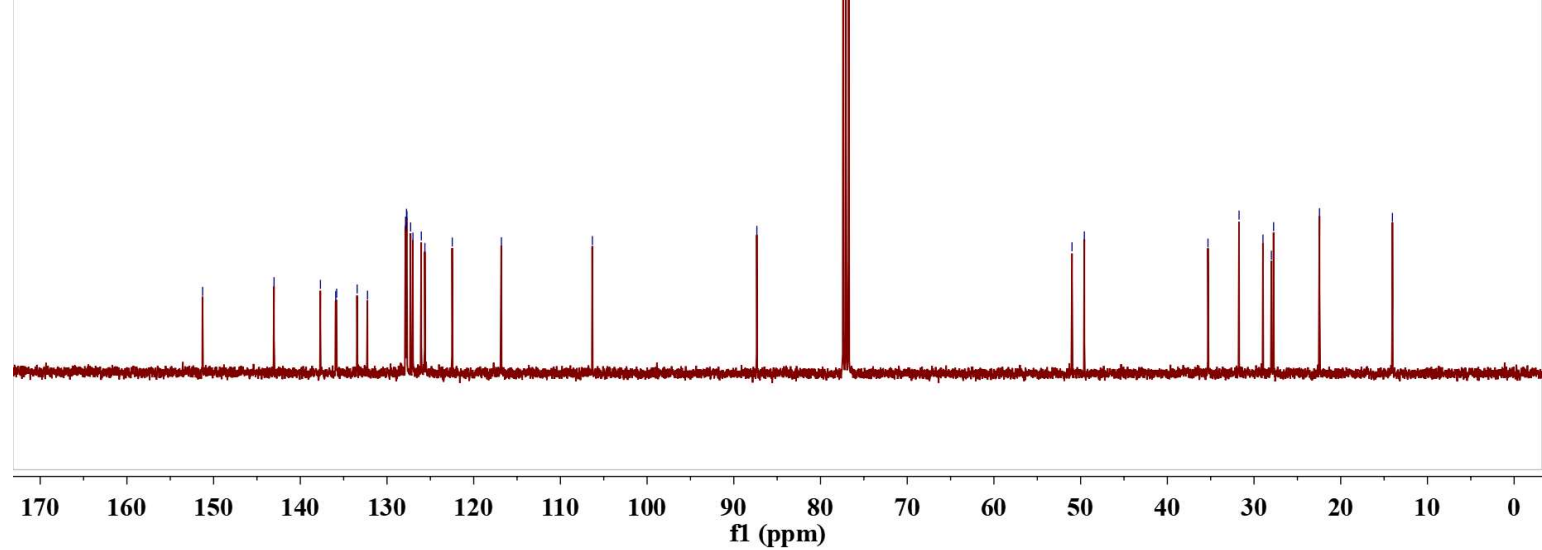


\section{8am}

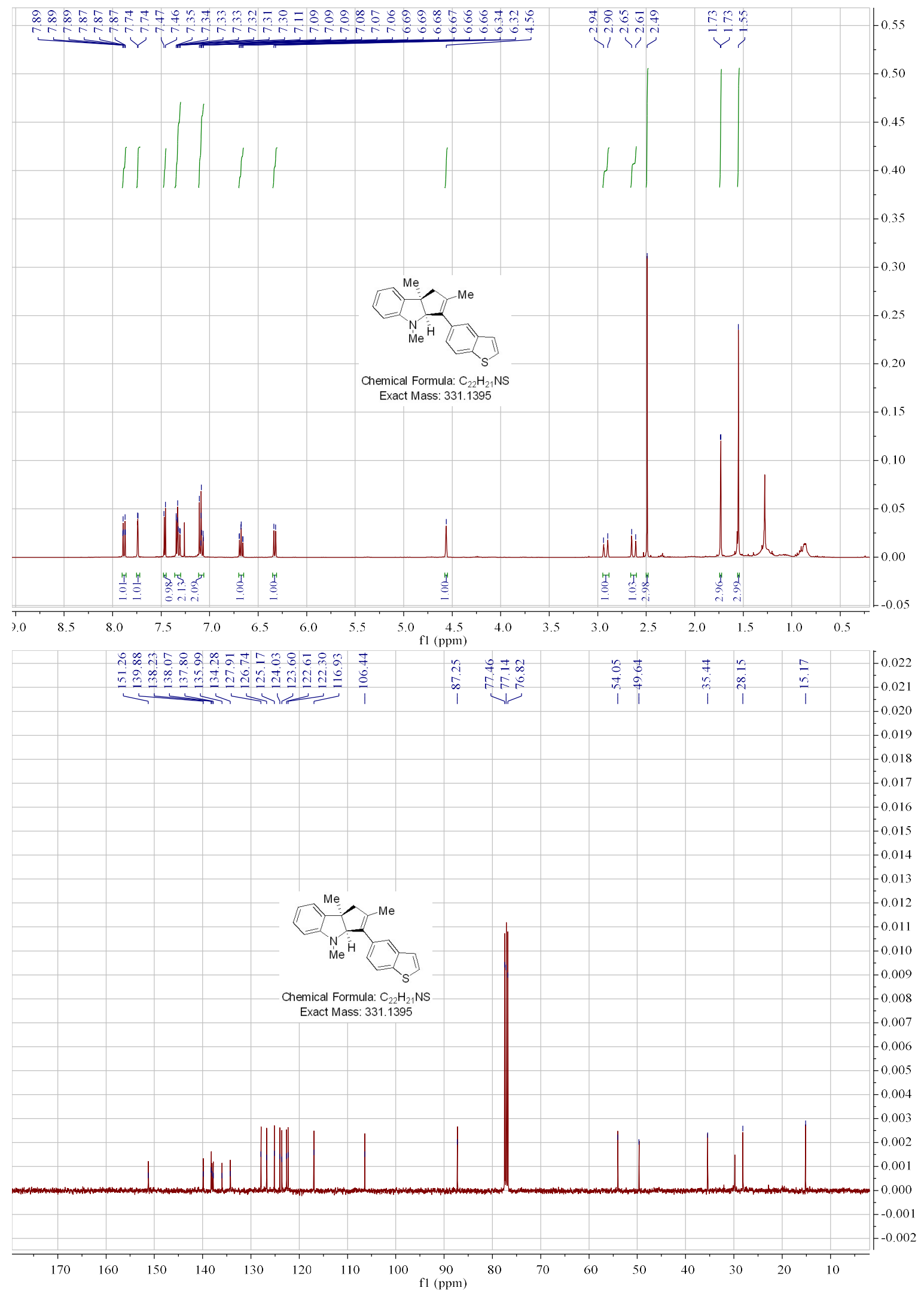




\section{8an}

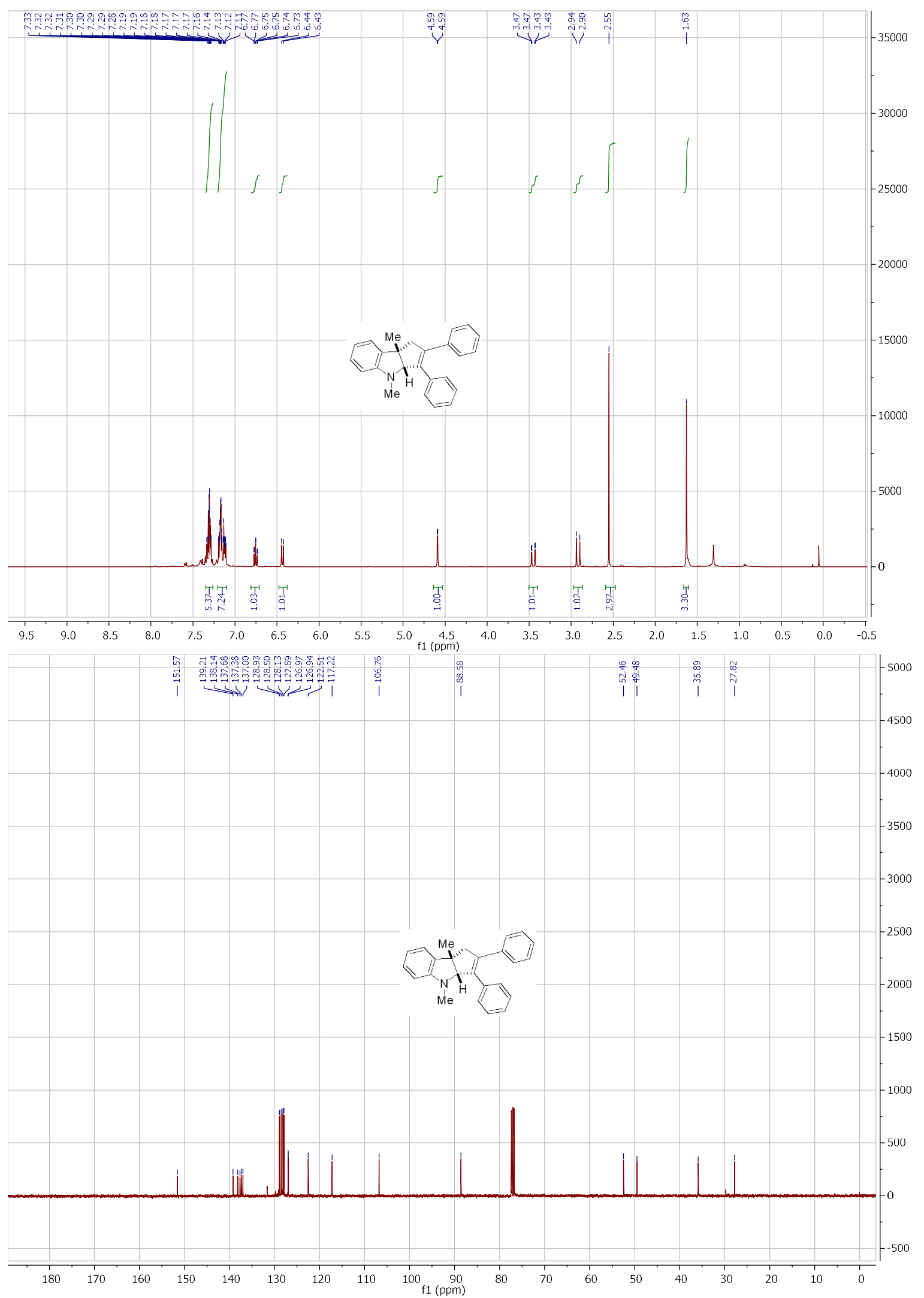




\section{$8 a o$}
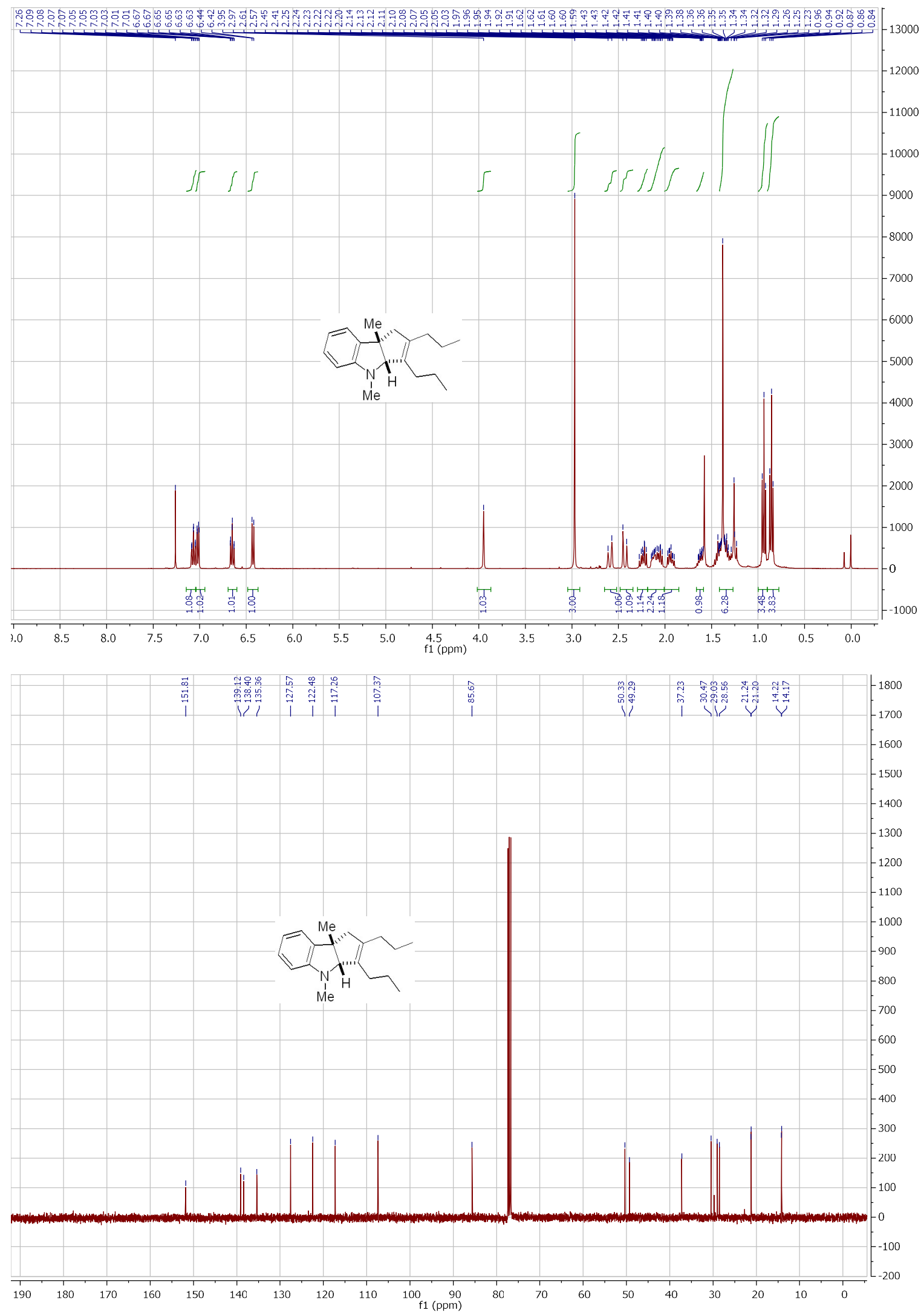


\section{References}

(1) Wang, K.; Ding, Z.; Zhou, Z.; Kong, W. Ni-Catalyzed enantioselective reductive diarylation of activated alkenes by domino cyclization/cross-coupling J. Am. Chem. Soc. 2018, 140, 12364.

(2) (a) Kong, W.; Wang, Q.; Zhu, J. Synthesis of diversely functionalized oxindoles enabled by migratory insertion of isocyanide to a transient $\sigma$-alkylpalladium(II) complex. Angew. Chem. Int. Ed. 2016, 55, 9714. (b) Kong, W.; Wang, Q.; Zhu, J. Water as a hydride source in palladium-catalyzed enantioselective reductive Heck reactions. Angew. Chem. Int. Ed. 2017, 56, 3987. (c) Liu, X.; Ma, X.; Huang, Y.; Gu, Z. Pd-Catalyzed HeckType Cascade Reactions with N-Tosyl Hydrazones: An efficient way to alkenes via in situ generated alkylpalladium. Org. Lett. 2013, 15, 4814. (d) Vachhani, D. D.; Butani, H. H.; Sharma, N.; Bhoya, U. C.; Shah, A. K.; Van der Eycken, E. V. Domino Heck/borylation sequence towards indolinone-3-methyl boronic esters: trapping of the $\sigma$-alkylpalladium intermediate with boron. Chem. Commun. 2015, 51, 14862.

(3) Zhang, Y.; Hsung, R. P.; Tracey, M. R.; Kurtz, K. C. M.; Vera, E. L. Copper sulfatepentahydrate-1,10-phenanthroline catalyzed amidations of alkynyl bromides. Synthesis of heteroaromatic amine substituted ynamides. Org. Lett. 2004, 6, 1151.

(4) Cruz, F. A.; Dong, V. M. Stereodivergent coupling of aldehydes and alkynes via synergistic catalysis using Rh and Jacobsen's amine. J. Am. Chem. Soc. 2017, 139, 1029. 